

\section{Anthropocene Islands Entangled Worlds}

Jonathan Pugh and David Chandler 
'A must read ... In this long-awaited book, [Pugh and Chandler] open up a new analytical agenda for the Anthropocene, coherently drawing out the power of thinking with islands.'

-Elena Burgos Martinez, Leiden University

'This is an essential book. By thinking with islands, Pugh and Chandler articulate new ontologies and epistemologies to help us understand the relational entanglements of the Anthropocene. The four analytics they proposeResilience, Patchworks, Correlation, and Storiation-offer both a critical agenda for island studies and compass points through which to navigate the haunting past, troubling present, and precarious future.'

\section{-Craig Santos Perez, University of Hawai'i, Manoa}

'All academic books should be like this: hard to put down. Informative, careful, sometimes devasting, yet absolutely necessary - if you read one book about the Anthropocene let it be this. You will never think of islands in the same way again'

-Kimberley Peters, University of Oldenburg

'Makes the compelling case that islands have never been merely geocultural objects of study, but rather, generative conceptual "objects" [for understanding and engaging] the wider, planetary, relational matrix within which the conditions of the Anthropocene era were created.'

-Michelle Stephens, Rutgers University

'What if we were to start not with the great drama of the world's falling apart, but with a myriad of smaller stories of its coming together? ... a unique journey into the Anthropocene. Critical, generous and compelling.

\section{-Nigel Clark, Lancaster University}

'Replete with "aha!" and "huh!" moments, this book offers insights for all of us ... who may not have recognised ... the value of "thinking with" islands more purposively'

-Lauren Rickards, RMIT University

‘... a must-read ... elucidates novel understandings of islands not only as patches of intensified Anthropocene proliferation, but as sites to examine the intricate relationships between life, matter, and meaning in a changing world.

\section{-Adam Searle, University of Cambridge}

'Anthropocene Islands establishes Pugh and Chandler as two critical and agenda-setting thinkers within island scholarship ... [It] cogently argues that islands have become emblematic figures of the Anthropocene and are moreover influencing the manner in which Anthropocene thinking is developing. ... a timely and essential contribution ...? 


\title{
Anthropocene Islands
}

\author{
Entangled Worlds
}

Jonathan Pugh and David Chandler

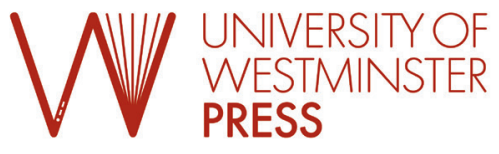

University of Westminster Press www.uwestminsterpress.co.uk 


\author{
Published by \\ University of Westminster Press \\ 101 Cavendish Street \\ London W1W 6UW \\ www.uwestminsterpress.co.uk
}

Text $($ ) Jonathan Pugh and David Chandler

First published 2021

Cover image: (c) Oliver Chandler

Print and digital versions typeset by Siliconchips Services Ltd.

\author{
ISBN (PDF): 978-1-914386-01-5 \\ ISBN (EPUB): 978-1-914386-02-2 \\ ISBN (Kindle): 978-1-914386-03-9 \\ ISBN (Paperback): 978-1-914386-00-8 \\ DOI: https://doi.org/10.16997/book52
}

This work is licensed under the Creative Commons AttributionNonCommercial-NoDerivatives 4.0 International License. To view a copy of this license, visit http://creativecommons.org/licenses/by-nc -nd/4.0/ or send a letter to Creative Commons, 444 Castro Street, Suite 900, Mountain View, California, 94041, USA. This license allows for copying and distributing the work, providing author attribution is clearly stated, that you are not using the material for commercial purposes, and that modified versions are not distributed.

The full text of this book has been peer-reviewed to ensure high academic standards. For full review policies, see: http://www.uwestminsterpress.co.uk/site/publish/

Suggested citation: Pugh, J. and Chandler, D. 2021. Anthropocene Islands: Entangled Worlds.

London: University of Westminster Press.

DOI: https://doi.org/10.16997/book52. License: CC-BY-NC-ND 4.0

To read the free, open access version of this book online, visit https://www .uwestminsterpress.co.uk/site/books /10.16997/book52 or scan this QR code with your mobile device:

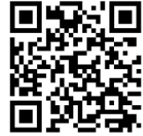


Shall we make 'island' a verb?

(Teresia Teaiwa, 2007: 514) 



\section{Contents}

Preface and Acknowledgements ix

1. There are Only Islands After the End of the World 1

2. Resilience: The Power of Interactive Life 41

3. Patchworks: The Ontology of the World 69

4. Correlation: Registers of Change 109

5. Storiation: Holding the World 141

6. Conclusion: A Critical Agenda for the Anthropocene 179

References 193

Index 225 



\section{Preface and Acknowledgements}

\section{Preface}

The island has a new found prominence and has become one of the most recognised geographical forms of the Anthropocene. It is a powerful symbol of such forces as global warming, rising sea levels, the fallouts of nuclear proliferation, ocean acidification, the waste of consumerism, ongoing colonialisms, changing ecologies and evolutionary pathways, disruptive weather patterns, including intensified hurricanes and cyclones, and much more besides. But the key argument of this book is that not only does contemporary scholarship and associated practice regularly write about islands, they draw upon and think with islands as key sites for working through today's overarching problematic of relational entanglements, awareness and feedbacks. Our claim is that islands and islanders appear so often - and in so many different ways - in the development of relational thought associated with Anthropocene approaches, as to make the questions 'why?' 
and 'how?' worthy of more focused attention. Islands not only appear in the works of scholars of islands, policymaking, activism or artistic practices explicitly directed at islands or islandness, they also increasingly appear, implicitly or apparently in the 'background', as sites or locations in broader Anthropocene research. Anthropocene Islands articulates why it matters that work seeks to bring island characteristics - or what we might call the generative force of 'islandness' - to the forefront for thinking through the Anthropocene.

This book schematically draws out why and how contemporary thinking engages and works with islands. Our key argument is as follows: The human/nature divide of modernity is today frequently argued to have collapsed and work on the Anthropocene intensively focuses upon the new problematic of how humans are relationally entangled with the more-than-human forces of transformative planetary changes. Debates about the Anthropocene are characterised by this central and overriding interest in relational entanglements, interactions and feedbacks (Colebrook and Weinstein, 2017; Colebrook, 2019; Giraud, 2019). It is therefore unsurprising that scholars, artists, poets, activists, policymakers and practitioners would want to engage particular geographical forms which exemplify these new stakes. Anthropocene Islands: Entangled Worlds analyses the powerful pull of islands as a figure for thought. They are a key figure for work on the Anthropocene precisely because islands are widely understood to be generative and productive for the core concerns of contemporary thinking.

This is of course not to deny the importance of other forms which are also being widely engaged in Anthropocene thinking. Rather, it is to foreground that thinking and working with islands has enabled Anthropocene thinking to cohere and be applied well beyond its originary fields as more and more physical and geographic entities, from the microbiome of the human gut to oceans and rainforests, are similarly explored as relational systems. Work with islands has emerged as particularly important for the key problematics of Anthropocene thinking. However, to date, no 
book has analysed why and how broader Anthropocene thinking works with islands for the development of relational ways of being (ontology) and knowing (epistemology). This book seeks to begin to fill this gap in the literature.

We do not argue that Anthropocene thinking is determined by the modes of its island production or engagement, nor indeed by its production via work with any other geographies. Rather that, given the many and various ways in which islands are being engaged in the development of Anthropocene thinking in so many publications, policy-developments and critiques - both explicitly and implicitly - this warrants further, close attention. We believe that it is not only useful to examine the more immediate or 'surface' focus of any work or publication, but also to slow down, to drill down further, as we have tried to do in this book, in order to draw out how islands, whether overtly or tacitly and across a range of contemporary works - have generated new or alternative approaches to being and knowing in the Anthropocene. It is these concerns that this book brings to light.

To date, a lot of research has been undertaken on how modern frameworks of reasoning reductively grasp and work with islands to produce certain understandings of them; for example, as sites of 'simplicity' or as 'laboratories'. As many have now pointed out, these tropes were - and continue to be - important in enabling modern thinking, just as they are enabling of certain ways of thinking about islands (Gillis, 2004; McMahon, 2016; Crane and Fletcher, 2017; DeLoughrey, 2019). But, we think, there is now a need to more explicitly focus and turn to how other contemporary debates produce and are shaped by various understanding of islands as liminal sites of modernity, key sites of 'relational entanglements' in the Anthropocene, and how these bring to the fore the generation of different relational ways of being and knowing as well. To do otherwise would be to once again risk returning to the notion that islands are merely 'blank spaces' for the development of contemporary thought. It would be to suggest that there really is a human/nature divide where the world, and how we think about various peoples, like islanders, who live in it, plays no part 
whatsoever in the generation of thought. How we think about and engage geographical forms and associated cultures matters for the development of thought in the world.

Equally importantly then, just as we believe work with islands does matter for the development of contemporary thinking, we do not think that this is because of some sort of deterministic, essentialised or realist island ontology. To understand that there is no human/nature divide is also to understand, as Whitehead (1968, $1967,1985)$ would say, that both are produced and exist together immanently in the world. This is why, we think, it is important to do the detailed work of examining and analysing the many, different and various ways in which islands are being brought into play, for the development of relational ontologies and epistemologies associated with Anthropocene thinking. This is why we take an analytical approach; less one of advocating what island thinking and practices should be, and more about heuristically drawing out and analysing the ways in which these conceptualisations are today being developed.

This book can be understood as part of a wider 'Anthropocene Islands' ${ }^{1}$ project and network currently being developed; building collaborations between a range of scholars, writers, artists, poets and others, around an agenda for island studies in the Anthropocene. In our own contribution to this project, in the following pages, we present a way of breaking down island thinking in the Anthropocene and tracing the developments of this work, as islands increasingly come to stand in for the world itself. We do this by separating work that focuses on acting on the world and thus has a greater concern with ontology - focusing on underlying forms of relationality and interconnection - and those with a greater concern for epistemology, i.e. ways in which we can come to know via the changing appearances of the world.

With regard to how islands come to be imagined and to be seen as contributing to thinking of the Anthropocene ontologically, we highlight a continuum from the relatively more traditional or modernist relational ontology of 'Resilience', where islands of interactive relation take clear system-like forms, to what we call 
'Patchworks', where islands compose the world in infinite depth and complexity. With regard to island-thinking as Anthropocene epistemology, we establish and analyse a similar continuum, from 'Correlation', where systemic regularities enable non-causal forms of knowledge through registering changes in systems of relation, to 'Storiation', where modes of registration and relation disrupt linear understandings of time and space. We explore these analytics in the chapters that follow.

\section{Acknowledgements}

This book has been four years in the making. Some of the work that has been preparatory for our project has been published in earlier forms. For example: our working through of some of the limits of relational and resilience approaches, 'Islands of Relationality and Resilience: The Shifting Stakes of the Anthropocene', was published in Area: Journal of the Royal Geographical Society 52(1), 2020; an early version of our ideas around the analytic of Correlation, central to Chapter 4, 'Islands and the Rise of Correlational Epistemology in the Anthropocene: Rethinking the Trope of the "Canary in the Coalmine" was published in Island Studies Journal (published online July 2020); and the broad conceptual framing of our project, reworked in Chapter 1, 'Anthropocene Islands: There are only islands after the end of the world', is forthcoming as the introduction to a forum discussion in Dialogues in Human Geography.

During this process we have benefitted significantly from a wide range of discussions with colleagues, friends and family. We would like to thank our families for their love and support most of all. Jonathan would like to thank Lara, Eva, Zoe, John and Val and David would like to thank Harvey, Oliver, Paulina and Camden.

We also would like to thank Godfrey Baldacchino, Adam Grydehøj, Ilan Kelman, Michelle Stephens, Yvonne Pugh, Sue Searle, Jim Bohland, Phil Hayward, Brian Russell Roberts, Everton Hinds, Kevin Grove, Mimi Sheller, Liz DeLoughrey, Elaine Stratford, Laurie Brinklow, Lisa Fletcher, Eric Clark, 
Huei-Min Tsai, Johannes Riquet, Elizabeth McMahon, Yolanda Martínez-San Miguel, Daniel Graziadei, Kasia Mika, Elena Burgos Martínez, Pippa Marland, Sun-Kee Hong, the Analogue University, Helen Dawson, Nick Megoran, Raksha Pande, Rosa Enn, Beth Robertson, Ben Bowsher, Steve Aggett, Adge Aggett, Ross Aggett, Mim Aggett, Lindsay Bremner, Christian Depraetere, Steve Royle, Sasha Davis, Jan Petzold, Pete Hay, Jenny Isaacs, Britta Hartmann, Karen Salt, James Ellsmoor, Julian Reid, Grant McCall, Rosie Alexender, Lorraine Levey, Ron Levey, Kevin Morrow, Helen Morrow, Evangelia Papoutsaki, Beate Ratter, Annette Breckwoldt, Carol Farbotko, Lauren Rickards, Phil Steinberg, Kimberley Peters, Susan Mahon, May Joseph, Yaso Nadarajah, Sarah Nimführ, Stephanie Wakefield, Jennifer Lawrence, Chantal Mouffe, Maddy Thompson, Malachi McIntosh, Rebecca Hester, Crispin d’Auvergne, Glenn Loughran, Christine Haimann, Celina Vale, Veena Das, Didier Fassin, Alastair Bonnett, Bojan Fürst, Stuart Dawley, William Maloney, Greca Meloni, Anna Kouremenos, Sean Dettman, Peter Hopkins, Laura Dierksmeier, Al James, Craig Santos Perez, Elia Apostolopoulou, John Forester, Carl Olsson, Patsy Healey, Jean Hillier, Erin Henderson, Aideen Foley, Stefano Moncada, Stacey Macdonald, Dani Redd, Harshavardhan Bhat, Amina Ghezal, Emanuela Borgnino, Maggie Jane Whitten Henry, Janet Momsen, Kevon Rhiney, Anna Baldacchino, Dunja Fehimović, Katherine Sammler, Rory Rowan, Pamela Richardson, Sanne Rotmeijer, the Executive Committee of the International Small Island Studies Association (ISISA), the International Geographical Union (IGU) on Islands, and the Editorial Boards of Island Studies Journal and Shima.

\section{Notes}

${ }^{1}$ The 'Anthropocene Islands' project can be found at https://www .anthropoceneislands.online. Through agenda-setting publications and talks, a monthly reading group, an ongoing section of Island Studies Journal, an early career analytical study space, workshops and sessions at conferences, the 'Anthropocene Islands' initiative will 
examine why and how islands are being engaged in the development of Anthropocene thinking (particularly with regard to relational approaches to contemporary thought).Please email Jonathan.Pugh@ ncl.ac.uk if you are interested in being involved in the Anthropocene Islands' project. 



\section{There are Only Islands After the End of the World}

\section{Introduction: Thinking with Islands in the Anthropocene}

Many Anthropocene scholars provide us with the key take-home message that they are writing 'after the end of the world' (Morton, 2013; Tsing, 2015; Danowski and Viveiros de Castro, 2016; Watts, 2018; and Gumbs, 2018 are just some examples). Not because they are necessarilywriting aboutapocalypse, butbecause theyareengaging the Anthropocene after the profound crisis of faith in Western modernity that has swept across academia in recent decades. For these contemporary thinkers, artists, activists, poets, policymakers, and many others besides, modern frameworks of reasoning which claimed to separate out humans from nature - to be able to grasp the 'world' as a coherent, controllable and manageable object - are part of the problem rather than the solution (Latour, 2017; Yusoff, 2018). In the Anthropocene, relational entanglements are understood to be too rich, vibrant and complex to be commanded in this modern way (Alaimo, 2016; Haraway, 2016). Indeed, it is widely noted that the question of 'relational entanglements'

How to cite this book chapter:

Pugh, J. and Chandler, D. 2021. Anthropocene Islands: Entangled Worlds.

Pp. 1-39. London: Ubiquity Press. DOI: https://doi.org/10.16997

/book52.a. License: CC-BY 4.0 
is the problematic of contemporary thinking (Daou and PérezRamos, 2016; Hamilton, 2017; Colebrook and Weinstein, 2017; Colebrook, 2019; Giraud, 2019).

Here, Derrida (2011: 9) resonates powerfully when he says that once faith in modern reasoning collapses we are faced with the stark realisation that ' $\mathrm{t}$ ] here is no world, there are only islands'. Derrida proposed deconstruction as a method or approach for challenging the metaphysical claims of modern philosophy. For Derrida, islands were key framing devices because they are the most obvious spaces of disruptive relations which work against modernity's grasping or appropriative approach to the world and its metaphysical grounding propositions. This book expands upon Derrida's observation to analyse how work with islands has become productive in the development of many of the core conceptual frameworks for Anthropocene thinking. Islands have become important liminal and transgressive spaces for work on the Anthropocene, both inside and outside the modernist world, both real and imagined, from which a great deal of Anthropocene thinking is drawing out and developing alternatives to hegemonic, modern, 'mainland' or 'one world' thinking.

If we were to summarise the contemporary shift towards working with islands in a set of concepts it would be those of 'relational entanglements', 'relational awareness' and 'feedbacks'. These are the key tropes of this book. As we explore throughout, in contemporary debates about the Anthropocene, islands are regularly invoked as having a different set of capacities, affordances and potentialities to modern or mainland life. The widely heralded capacity of islands to respond to the environment, as shaped by relational agency, is the key way of understanding why islands have become significant for so much contemporary thinking (Bahn and Flenley, 1992; Eriksen, 1993; McMillen et al, 2014; Robertson, 2018; Watts, 2018; Barad, 2019; Dawson, 2019). Islands exemplify how all life in the Anthropocene is relationally entangled and co-dependent (Morton, 2016a; Wolfe, 2017). For those concerned with the hubris and counterproductive nature of modern frameworks of reasoning, the problem is their neglect 
of relationships and their narrow focus upon essences and linear or universal causality. The relations and feedback effects associated with the Anthropocene are widely held to be masked by and hidden from a reductionist modern ontology and epistemology (Nancy, 2014; Colebrook, 2016; Clark and Yusoff, 2017). Thus, working with islands plays an increasingly notable role in Anthropocene thinking as it is precisely with islands that these relational effects come to the fore (Handley, 2015; Paravisini-Gilbert, 2015; Ingersoll, 2016; Camus, 2018; Wu et al, 2019; Elias, 2019). Islands are an attraction and lure for contemporary scholarship which seeks to challenge the hubris of modern frameworks of reasoning (Percival, 2008; De Souza et al, 2015; Tsing, 2015; Morton, 2016a; ecoLogicStudio, 2017a; Hayward, 2018; DeLoughrey, 2019; Suliman et al, 2019; Perez, 2020a; Clark and Szerszynski, 2021; Burgos Martinez, 2020; forthcoming). In modernity, the separateness, isolation and the relational dependencies of islands appeared to be their weakness, holding back island development and productiveness. However, these relational sensitivities are, today, understood by many to be key to planetary survival (Teaiwa, 2007; Kueffer and Kaiser-Bunbury, 2014; Bird Rose, 2017a; 2017b).

We are not suggesting that there is such a thing as island thinking'; there are, of course, only variations in ways of drawing upon and working with islands in different places and at different times in history. Under older European and modern thought the island was often understood as insular, isolated, liminal or backward, even populated by savages, when compared to continental, mainland reasoning (Malinowski, 1921; Grove, 1995; Glissant, 1997; Brathwaite, 1999; Gillis, 2004; Baldacchino, 2006; Royle, 2007; Olwig, 2007; McKittrick, 2006; McMahon, 2016; Crane and Fletcher, 2017; Riquet, 2020). Building directly from these older narratives, in debates about climate change, islands are still of course frequently reductively framed in Western and modern fantasies of control; understood as helpless, disposable or in need of saving by others (Farbotko, 2010; Cameron, 2011; Proctor, 2013; DeLoughrey, 2019). Here, islanders are 'often portrayed as passive victims waiting to be saved from their sinking islands' (Suliman et al, 
2019: 304). Yet, the configurations and stakes of debate are also rapidly changing as well. Thus, this book analyses how the island is being re-thought, worked with and drawn upon in the development of contemporary thinking. In particular, we are going to explore how islands have shifted from the margins and become more important to many international debates, precisely because, after the supposed closure of the modernist imaginary of progress, islands have emerged as key sites for understanding relational entanglements which have come to the forefront in the search for alternative forms of thought and practice in the Anthropocene.

With 'relational entanglement' widely understood to be the central problematic of the Anthropocene, specific geographical forms and cultures have come to the fore, which enable the drawing out and development of this key focus and concern. As Donna Haraway (2016: 57) says, ' [i]t matters which thoughts think thoughts. Compared to islands, other geographical forms, like valleys, deserts and mountains, seem less productive when it comes to working through the problematic of relational entanglements. These geographical forms therefore appear less often, while in contrast, the island has become arguably one of the most emblematic figures for debates about the Anthropocene and related forces such as global warming, rising sea levels, ongoing colonialisms, intensifying ecological degradation and species loss, the ecological effects of mainland Western consumerism, nuclear testing and fallout, changing weather patterns such as intensified hurricanes and cyclones, and ocean acidification, to name just a few examples (Haraway, 2016; Kelman, 2018; Fitzpatrick and Erlandson, 2018; Baldacchino, 2020).

The important contention of this book, however, is that the rise to prominence of islands in broader contemporary debates about the Anthropocene has not only come about because islands are high-profile symbols of transforming planetary conditions, or because islands might be understood as smaller and more manageable 'test tubes' for policy and scientific experimentation. Islands have long been understood conceptually and empirically, across a very broad range of disciplines, as important spaces for varying 
expressions of relational entanglements - from Darwin (2010) and Mead (2001), through to Strathern (2004), Glissant (1997), Walcott (1998), Hau'ofa (2008), Mitchell (1978), Condé (1992) and Brathwaite (1999). In many influential traditions of Western critical theory, including most notably those of Deleuze (2004) and Derrida (2011), the island has regularly been employed as a key figure which explicitly disrupts the grasp of modernist, linear and reductionist 'mainland' thinking. In island studies more generally, for many years now, a very broad range of island scholarship has understood islands as key 'relational spaces.'

This book analyses how islands are being worked with, thought about and engaged in contemporary approaches to the Anthropocene. We examine how the liminal figure of the island is significant in the development of new or alternative approaches to ontology and epistemology, distinct from modern, mainland, thought. Here we want to be clear about this central claim. Of course we are not saying that all Anthropocene thinking chooses to explicitly engage the geographical form of the island, but it seems very clear today that a concern with islands' relational entanglements, affordances and feedbacks, regularly surfaces and is conceptually deployed in debates about the Anthropocene, marking islands as particularly productive for Anthropocene thinking.

In researching this book it has become clear to us that the island cannot be understood as coming to the fore only after the development of new approaches, alert to seeing relational interdependencies. To simply focus upon the development of relational ontologies and epistemologies in the minds of Anthropocene thinkers would be to deny the important 'work' that particular geographical forms and cultures, such as those associated with islands, are doing in these developments. The ways in which islands are being thought about and conceptualised in these debates, we argue, is generative of relational thinking in the Anthropocene, rather than merely an example of its application. Thus, we do not only write about islands in the Anthropocene. Rather, Anthropocene Islands: Entangled Worlds' draws out heuristically and examines thinking with islands after the end of the world. ${ }^{2}$ Geography 
matters for the development of thought in the world. Put simply, there are not islands, on the one hand, and the human mind on the other. If we accept that developments in broader social and human thought, and the material world are not separate but profoundly interconnected (i.e. that there really is no human/nature divide), then islands can be understood as important seeds for the conceptualisation of the Anthropocene; a liminal entry point for wider contemporary forms of thought. This is the generative power and lure of working with islands for Anthropocene thinking.

Whilst, as we will consider, the recognition of the conceptual generative power of islands has been influenced by prominent island scholarship and research itself around the world going back many decades, we cannot understand the broader shifts taking place in Anthropocene thinking without also addressing the important place of contemporary shifts in the Western academy as well. Bruno Latour (1993) has argued that 'We Have Never Been Modern'. Whether that is true or not for the Western, mainland subject Latour was writing for, depends upon how literally we take the modernist imaginary. What is true is that islands 'were never modern' in the Western imaginary in the particular sense that islands, by definition, imply a series of separations that, throughout the history of modernity, were seen to cut off islands from mainlands (Gillis, 2004). Island scholars regularly make the point that islands have often symbolised a 'difference' or 'otherness' which stems from this separation (Glissant, 1997; Beer, 1997; Brathwaite, 1999; Baldacchino, 2006; Grydehøj, 2017). Under frameworks of modernity, islands were frequently seen to be lacking the essence of European 'mainland' forms of being which were cast in terms of civilisation, progress and advancement (Edmond and Smith, 2003; McMahon, 2016). While these attributes were considered as positive in modernity, island being was, by contrast, seen as 'backward', 'closer to nature' or 'slower.' Yet today, as we argue in this book, with modernist assumptions being profoundly questioned in a world of global warming, catastrophic climate change and species extinction, island 'differences' - the attributes, relational affordances and powers associated 
with islands - have put working with islands to the forefront of the Anthropocene. ${ }^{4}$

The purpose of this book is to conceptually clarify and draw out this shift. Working with islands or relational thought per se is not one homogenous 'other' to modernist or mainland approaches, and so it is important to start a conversation about how we engage in working through the rich variety of possibilities and opportunities that island-oriented approaches afford today. In order to initiate this process, we carve out four tendencies or analytics which position the figure of the island within broader debates: these we categorise in terms of 'Resilience' (discussed in Chapter 2), 'Patchworks' (Chapter 3), 'Correlation' (Chapter 4) and 'Storiation' (Chapter 5). ${ }^{5}$ They mark out two sets of conceptual sliding scales which, in the first half of the book, focus upon ontology (Resilience and Patchworks), and, in the second half, ontoepistemology (Correlation and Storiation). By using a conceptual sliding scale or continuum, we seek to illustrate how Anthropocene thinking emerges as a distinct set of ontological and ontoepistemological approaches, increasingly losing its modernist constraints. Thus, 'Patchworks' can be seen as expanding and reworking the island thinking which informs 'Resilience' ontologies; and the same can be said of how the onto-epistemology of 'Storiation' reworks that of 'Correlation'.

Throughout the book, we analyse how the emergence of these four analytical framings draws heavily upon islands as a reserve for non-modern imaginaries, of forces of relation and feedback, and of possible alternative ways of working and conceptualising that go beyond the limits of modernist framings of linear causality, universality and homogeneity. Heuristically working across a wide spectrum of authors and works, we gather a range of key examples, in order to illustrate how island imaginaries of human/ world relations are shaped in non-modernist ways in the Anthropocene. In the conclusion (Chapter 6), we consider how our initial set of four analytic distinctions could facilitate discussion around a critical agenda for contemporary island studies. Thus, we see this book as the starting point for a broader project - which we are 
calling 'Anthropocene Islands' - focusing upon conceptually and heuristically exploring the stakes of island-work for contemporary thought and the Anthropocene problematic. To be absolutely clear, as we stated in the Preface, we do not see what we present in this book as the closure of the project but rather as the initial opening for a critical agenda which we seek to develop over the coming years.

\section{Relational Ontology}

As just noted, the approaches to relational ontology examined in this book are those of 'Resilience' (Chapter 2) and what we call 'Patchworks' (Chapter 3). As a brief summary of their key characteristics before we get into the details: Resilience, as an expression of working with islands, draws out how the resilient capacities of island life and islanders are part and parcel of spatially and temporally fixed assemblages with autonomous capacities for self-ordering or adaptation. Resilience thus traces and responds to relational entanglements, affordances and feedback effects over space and time as constituted in Newtonian or Euclidian geometry. Central here is how Resilience thinking draws upon the immanent interactive powers of life itself - exemplified in many of these debates by island life - as a self-regulating system. As we will shortly elaborate, for us this is a key reason for islands and islanders' emergence as high-profile agential spaces for Resilience thinking in debates about the Anthropocene (McMillen et al, 2014; Petzold and Ratter, 2015; Raygorodetsky, 2017; Pugh, 2017; Nicks, 2017; Chandler and Pugh, 2020a; 2020b; Kelman, 2020; Pugh, 2018; Baldacchino, 2018; Camp et al, 2019). Because, as the nineteenthcentury naturalist Charles Darwin brought to popular attention, islands are remarkable localised sites of relational entanglements and feedback effects.

What we are calling 'Patchwork ontologies', which we see as an intensification and development of the key relational focus of Resilience, can be located towards the other end of this ontological continuum or sliding scale. Patchwork approaches are becoming 
increasingly prevalent in debates about the Anthropocene, drawing heavily upon the powers of islands, foregrounding ontological tropes of relational entanglement and feedback effects. But, in contrast to Resilience, Patchwork approaches tend to have an open ontology of spatial and temporal becoming. They do not draw so much upon an imaginary of islands existing as selfregulating systems, tracing continuities in relation across linear time into an ever more efficient order, as in Resilience. Rather, towards the other end of the spectrum, Patchwork ontologies accept the Anthropocene as a condition which we are all already in. They actively and productively 'stay with the trouble' (Haraway, 2016) of relational disturbances and emergent effects (Tsing, 2015; Daou and Pérez-Ramos, 2016; Herrington and Lokman, 2016; Bird Rose, 2017a; 2017b; Watts, 2018), affirming the creative possibilities of a world no longer bound or constrained by the modernist imaginary.

We highlight the importance of working and thinking with islands for the development of Patchwork approaches via a wide range of examples, examined in Chapter 3. These include, among many others, Anna Tsing's (2015) engagements with Japanese islanders' practices; Deborah Bird Rose's (2017a; 2017b) work with the Aborigines of Australia; Phil Hayward's (2012b) with Haida Gwaii; Daniel Daou and Pablo Pérez-Ramos' (2016) with island thinking in contemporary design; Mimi Sheller's (2020) with local Caribbean island practices; Brian Russell Roberts and Michelle Stephens' (2017) with the 'anti-explorer' method; Teresia Teaiwa's (2007) islanding as a 'verb'; Juliana Spahr's (2005) poetry about Hawai'i; and Laura Watts' (2018) engagements with Orkney islanders. Our argument throughout is that it matters that authors choose to engage and draw heavily upon islands and islanders. Invoking certain island imaginaries - and islands' relational entanglements, affordances and feedbacks in particular - is generative for such Patchwork approaches. These, as we examine, focus upon engendering or inculcating other ways of becoming than those of Resilience. Islands are not worlds to be managed or adapted to but instead become a powerful way of 
expressing, opening up and understanding non-modern processes of world-making.

\section{Resilience - Chapter 2}

Turning to the details of these relational ontologies, Chapter 2 examines the heuristic of 'Resilience', which we articulate here as the field through which island ontologies have been most obviously adopted by mainstream academic and policy-thinking (Briguglio and Kisanga, 2004; Alliance Magazine, 2012; Baldacchino, 2018; Pugh and Chandler, 2020a; Grydehøj and Kelman, 2020; Kelman, 2020). Resilience is conceptualised by us as an analytical field through which islands have emerged prominently in postmodern or non-modern framings of governance, as an alternative to linear thinking about progress and sustainability in the Anthropocene. Resilience seeks to capture the art of adaptation or of adaptive change in relation to changing circumstances (Grove, 2018; Anderson et al, 2020; Wakefield, 2020). At its most fundamental level, it presupposes a generative or productive relation across and within actors and agencies - not the importing of resources or external assistance - so it is the relational or contextual powers and affordances of these actors and agencies which are the key strengths to draw upon. Chapter 2 thus examines how, whereas modernity is seen to homogenise and reduce life to the lowest common denominator, repressing any form of being outside the norm, by contrast, drawing upon, engaging and working with islands has been significant to the rise of Resilience thinking because islands are imagined to have the opposite powers: the powers of creative and productive differentiation and individuation.

What enables islands to intensify relationalities, differentiation and individuation? Here Chapter 2 turns to the work of Charles Darwin, and the power he attributed to islands in his paradigmshifting perspective of life itself; not only exemplified, but revealed to the rest of the world, by island life. Darwin famously theorised the radiating vibrancy of life in the form of a branching evolutionary 
tree, where different environmental opportunities enabled different answers to the problems of life. Species evolved and adapted differently on the Galápagos because different island ecologies facilitated and enabled this differentiation. The key word for Darwin was thus 'divergence' (Quammen, 2018a: 6), which emerged from the separation and bounded nature of islands, and in focusing upon this he drew attention to how islands are powerful differentiating 'engines' for life itself. Darwin highlighted how islands reveal how all life is interactive and profoundly relational, with each island context drawing out different potentials. Darwin was obsessed with the power of islands - this 'island effect'. Thus, with mockingbirds:

These gray, long-beaked birds differed from island to island but so subtly that they seemed to have diverged from one stock. Diverged? Three kinds of mockingbird? Varying slightly, this island to that? Yes: they appeared distinct but similar, in a way that suggested relatedness. If that impression were true, Darwin confided to Henslow [his Cambridge biology professor], confessing an intellectual heresy, 'such facts would undermine the stability of the species'. (Quammen, 2018a: 4)

Darwin's work on islands brought attention to the differentiating, creative and adaptive potentialities of life itself. For Darwin, cats on an island, like lizards on a tiny Croatian island, or the finches on the Galápagos, do not evolve to become better cats per se, but 'better cats for catting on that particular island' (Quammen, 2018a: 6). There is a 'law of adaptation' at work (Quammen, 2018a: 6). Darwin's heresy was to overturn the idea that evolutionary speciation is linear, or to do with the essence of cat-being, but rather non-linear, to do with the relational context of cat-emerging or cat-becoming. Species do not evolve in the sense of a linear telos of 'progress' (Quammen, 2018a: 6). Thus, as Riquet (2020: 246) says, Darwin brought about 'a radical change of perspective [about islands] ... a relational perspective.' 'Islands allow Darwin to imagine ... a decentred world in flux, a conglomerate of crisscrossing lines' (Riquet, 2020: 260). Since Darwin's time, island life has become a high-profile symbol of non-linear emergence 
and diversification because islands are seen to enable contexts to intensify and magnify interactive feedback effects (Kueffer and Kaiser-Bunbury, 2014). In this way, as Gregory Bateson (2000: $455,457)$ acknowledged, the subject of evolution is no longer an isolated or autonomous one but the 'organism plus environment' or 'organism-in-its-environment'.

Chapter 2 examines how these creative attributes of island life are seen to be important to Resilience-thinking, because they demonstrate that adaptation to change is not only possible but is an ontologically inherent power of life itself. Without Darwin's understanding of how (island) life itself works, resilience theories could not have emerged in the way that they did. Central for us is also how early case studies of resilience frequently started by examining island life (Gane, 1975; Waddell, 1975; O'Keefe and Conway, 1977; Westman, 1986; Kelman, 2020). As the highly influential resilience scholar C. S. Holling noted in an interview about the resilience programmes which he initiated: 'When we considered whether someone would be good for the programme, the first question we'd ask was "Is he/she good on islands?" (Alliance Magazine, 2012). ${ }^{6}$ Given the longstanding understanding of islands as both laboratories for Western science and as key sites of creative adaptation, relational affordances and feedback effects, it is not surprising that islands 'provided a significant part of the earlier baseline for understanding vulnerability and resilience' (Kelman, 2020: 10). Early resilience theories drew upon island research extensively; including in Fiji (Gane, 1975), the Caribbean (O'Keefe and Conway, 1977) and Papua New Guinea (Waddell, 1975). Foregrounding islands as intensive sites of relational entanglements, affordances and feedback effects, Westman (1986: 5), for example, noted that the prediction of resilient properties of ecosystems in the Mediterranean can be approached 'through knowledge of the autoecological adaptations of key species to the stressor, or through cumulative experience of the response to disturbance at the community level'.

The focus upon relational contingencies and emergence also crucially reverses the epistemological and governing hierarchies 
of island vs. mainland. It challenges the top-down, modern and external centralisation of knowledge and power off-island, instead switching to focus on the active possibilities and relational potentialities of (island) life itself. We explore how today it is commonplace for international policymakers, academics and practitioners to work with islands in these ways and for them to highlight how islands are a resource for generating new understandings and capacities for Resilience (McMillen et al, 2014; Petzold and Ratter, 2015; Raygorodetsky, 2017; Ellsmoor, 2019). It is important to be clear how island life is seen as an important resource for these authors. They do not mean that island life is a resource in terms of a pile of materials that can be catalogued, extracted or worked upon through new or more productive technologies (as in Robinson Crusoe's obsessively modern listing and recording of facts about the island he was castaway upon). Instead, they mean that island life is necessarily always in excess of being: i.e. that there is always an untapped potential to the relational entanglements, affordances and feedback effects of life itself - notable in island life - in the here and now.

We present and understand Resilience as an analytic that works upon these virtual potentialities of (island) life. Thereby seeking to direct, instrumentalise and governmentalise approaches, often illustrating how the resilient capacities of (island) life are part and parcel of whole (island) socio-ecological systems. It is this drive that has made commonplace the understanding that islanders' knowledge systems include 'valuable insights on seasonal cycles, ecological processes, and the management of biocultural diversity that are relevant at a broad scale for understanding resilience and adaptability to the social-ecological effects of climate change' (McMillen et al, 2014: 44). Island life is widely understood as constituting a living system that the rest of the world may learn from; exemplifying the creative potentialities or 'emergent' powers of life itself - 'system effects' - that cannot be accessed directly by way of modern frameworks of reasoning. Whilst the 'Resilience' paradigm proliferates across many disciplines and settings (see Chandler, 2014; 2018a; Evans and Reid, 2014; Pugh, 2014; Grove, 
2018; Wakefield, 2020), we examine how working with islands has historically been and today remains significant to Resilience as a key ontological framing for many concerned with contemporary thinking. As we have said above, not all Resilience thinking explicitly engages islands. Rather, it is that the island, as an important figure for working through the central problematic of relational entanglements, makes it particularly generative and productive for contemporary engagements with the Anthropocene.

\section{Patchworks - Chapter 3}

Chapter 3 turns to explore what we call 'Patchwork ontologies', which we draw out as a characteristic of the work of many scholars, experimental artists, designers and activists engaged with debates about the Anthropocene and who work with islands (examples include, among others, Spahr, 2005; Teaiwa, 2007; Hayward, 2012a; 2012b; Daou and Pérez-Ramos, 2016; Yountae, 2016; Tsing, 2015; Roberts and Stephens, 2017; Bird Rose, 2017a; 2017b; Wetlands Wanderers, 2018; Watts, 2018; Sheller, 2020). As Craig Santos Perez (forthcoming) saliently notes, islands 'have received unprecedented attention' in recent years, not only in mainstream policymaking and Resilience debates, but also in the work of many high-profile critical theorists, from Donna Haraway (Hadfield and Haraway, 2019) to Anna Tsing (2015). As an island scholar and poet, Perez is attuned to this 'hyper-visibility' of islands. This attention to islands is highlighted, foregrounding how even as islands may sometimes appear to be 'backdrops' or 'in the background' of critical developments in Anthropocene thinking, there is no denying that a great deal of contemporary critique, artist practice and activism is being developed from work on islands and with islanders. ${ }^{7}$

Compared to Resilience, an important point, for us, about Patchwork ontologies is that they shift the register of debate towards affirmation, accepting that we are all already in the Anthropocene. ${ }^{8}$ Patchwork approaches develop and transform relational ontology so that the modernist imaginary of islands existing in a flat, 
two-dimensional space, side-by-side, tracing continuities in relation across linear time, is replaced with a more open ontology of spatial and temporal becoming (Glissant, 1997; Last, 2017). While this remains a relational understanding, Patchwork ontologies are more disruptive, destabilising the 'solutionist' or instrumentalising aspects of Resilience; making Patchwork approaches more open, less governmentalising and human-centred. If Resilience approaches seek to conserve modernity in the face of transformative planetary change, then the work of what we call 'Patchwork ontologists' - such as Anna Tsing, Juliana Spahr, Deborah Bird Rose, Brian Roberts and Michelle Stephens, Gilles Clément, Phil Hayward, Mimi Sheller and Laura Watts - foregrounds how entanglements of relation are never fixed. They thereby disrupt modern and Euclidian notions of space-time in distinction to those of Resilience.

Rejecting those who reduce and homogenise debate to a coherently discrete or separated island-system of relations, Patchwork ontologies instead focus upon patchwork islands of disturbances and emergent effects forming in nodes or knots of assemblages across time and space. It is the focus upon the disruptive power and the intensification of relational disturbances and effects (rather than modern, flat notions of space-time) which marks out Patchwork approaches; and it means that they cannot be easily 'exported' as a set of instrumentalising techniques or practices, as in the comparatively managerial ontological imaginary of Resilience. Patchwork ontologies work with islands to reframe the stakes of engaging the Anthropocene more openly and radically than Resilience in Patchwork approaches the world dissipates into patchworks of islands of relational co-entanglements and affects, so that drawing upon islands in this way becomes the ontology of the world (which, as we shortly examine, enables Glissant (1997) to be read as one of the key early exponents of a Patchwork ontology).

For Patchwork ontologies, islands are not merely worlds that we are in; rather, as Glissant (1997), Tsing (2015), Roberts and Stephens (2017) and Bird Rose (2017b) variously draw out, islands are also ways of expressing and understanding our own processes of world-making. Thinking with islands then importantly 
becomes a 'verb'9 (Teaiwa, 2007: 514; see also Baldacchino and Clark, 2013; Yountae, 2016) and a practice of opening ourselves to relational affects and knots of co-relational entanglements, rather than one of Resilience which tends to reify the world and suborn us to it. In the Patchwork ontologies we discuss in Chapter 3 - such as Tsing's (2015) examination of Japanese islander satoyama practices, Hayward's (2012a; 2012b) conceptualisation of the 'aquapelago', Brian Roberts and Michelle Stephens' (2017) conceptualisation of the 'anti-explorer method', Bird Rose's (2017a) engagement with the Aboriginal islander aesthetic of 'shimmer', the Wetlands Wanderers (2018) 'Startling Adventures of RonR', and Spahr's (2005) This Connection of Everyone with Lungs about Hawai' $\mathrm{i}$ - the focus is upon how we make, explore and journey, rather than merely reflect upon and become more aware of our relational interconnections so as to become resilient.

Patchwork ontologies are highly pragmatic, whereby creative intermingling has results which are frequently surprising, and indeed often inspirational, rather than something intentional or governmentalisable. Importantly, Patchwork approaches do not draw upon an immanent dynamic or trajectory, as in the case of Darwin's understanding of the evolution of species on islands. Instead, they often emphasise the importance of 'staying with the trouble' (Haraway, 2016), as life - regularly exemplified in these developments by island life - becomes less predictable, confineable and graspable in the Anthropocene (Tsing, 2015; Watts, 2018). Again, our point is that the material world, and the geographical forms which rise to the surface in these debates, matter for the development of thought in the world (Whitehead, 1967; 1968 ; 1985). The central focus of Patchwork approaches is 'givingon-and-with' (Glissant, 1997: 142) the power of disturbances and emergent effects, where, in the work of many influential Anthropocene scholars, activists, artists and experimental designers examined in Chapter 3, island ontology becomes a key resource to draw upon and to stimulate thinking about how relationality is radically open and contains potentialities or possibilities which are beyond our capacities to predict or to control. 
Chapter 3 examines how a key resource for what we call Patchwork ontology is Glissant's (1997) seminal text Poetics of Relation. Here Glissant's (1997) argument is that life (exemplified for him, above all else, by island life) is a coming to consciousness within what he calls the opacity of 'Relation' ${ }^{10}$ Conceptually speaking, for Glissant (1997), Relation is not actually an entity as such which could be transparently grasped and instrumentalised. Relation is instead the very process or movement itself, living through and with the disturbances and effects - of colonial legacies, island geographies, oceanic currents, changing shorelines, up to and including elemental forces themselves - that are formed and continuously re-formed to make up (island) life. In Glissant's (1997: 33) work, which examined the Middle Passage, creolisation, and the Caribbean, he argued that these islands were 'explosive regions' where Relation is 'gathering strength'. For Glissant (1997: 191-192), modern, mainland frameworks of reasoning had reductively and oppressively focused on how it was possible 'to grasp' the world; so that 'the verb to grasp contains the movement of hands that grab their surroundings and bring them back to themselves' (exemplified for Glissant by the grasping hands of colonialism on islands). By contrast, Glissant's radically open engagement with 'Relation' pushes relational thinking with islands to the point that we can never stand outside and grasp; only ever live within and experience 'the texture of the weave', living with and through the turbulence and relational effects (Glissant, 1997: 190).

Thus, as Glissant (1997: 206) says, while walking along a beach in Martinique and looking out to St Lucia:

I have always imagined that these depths navigate a path beneath the sea in the west and the ocean in the east and that, though we are separated, each in our own Plantation, the now green balls and chains have rolled beneath from one island to the next, weaving shared rivers that we shall open up when it is our time and where we shall take our boats. From where I stand I see Saint Lucia on the horizon. Thus, step by step, calling up the expanse, I am able to realize this seabow. 
Here, Glissant is not saying that it is possible to grasp or 'stand outside' of the island, or the world, observing it from an objective or true position in time or space, as in the ways of modern, mainland, or Cartesian frameworks of reasoning (or, indeed, in the confined island imaginaries of Resilience thinking). Rather, Glissant is contemplating how the totality of Relation makes an impression and manifests locally; how island life, and his own life as someone from Martinique, emerges from this coming to consciousness in Relation (Burns, 2012; Dash, 2006; Pugh, 2016a). Glissant (1997: 142) advocates a poetics which seeks to dig deeper into the world through 'giving-on-and-with', challenging universal, generalising or transcendent totalities in its ever 'more stringent demands for specificity. Glissant's poetics is a practical one in which the subject is no longer an 'observer' of relations but practically worlding itself in a concrete, embedded and embodied way.

As Drabinski (2019: $\mathrm{x}$ ) says of Glissant's work, '[t]hinking in ruins, which is productive rather than (solely) melancholic, is already thinking the archipelago as a geography of the globe and the geography of thought'. For Glissant, the power and opening up of Relation is reflected particularly well in today's crisis of faith in modern reasoning which had sought to grasp, instrumentalise, command and control the world as a coherent and manageable object. Thus, at the end of the Poetics of Relation, touching upon contemporary debates about the Anthropocene by reflecting upon the fallout from Chernobyl, Glissant (1997: 202-203) says:

What was the infinite detour taken by this nuclear catastrophe, whose worldwide repercussions were felt among the destitute as well as among the well-to-do, in savanna villages, probably, just as much as in skyscrapers, and which consequently fed the most passively experienced of commonplaces in the planetary consciousness, that led it also to be condensed into what seemed to be an involuntary poem, through which it happened that the world could speak to us? The landscape forced its way through the dazzling barrier, fixing upon the superficial brilliance this terse scrap of utterance. ... The circle opens up once more, at the same time that it builds in volume. Thus, at every moment Relation 
becomes complete but also is destroyed in its generality by exactly what we put into action in a particular time and place ... We leave the matrix abyss and the immeasurable abyss for this other one in which we wander without becoming lost.

Glissant's work allows us to draw out some of the key elements or aspects of what we call Patchwork ontologies, discussed in Chapter 3, in which islands are no longer conceptualised as confined sites of fixed spatial differentiation and individuation. Instead, islands increasingly function as the ontology of the world; where the fallout of a nuclear plume, the Japanese islander satoyama practices discussed by Tsing (2015), the 'anti-explorer method' of Roberts and Stephens (2017), or the contemporary design processes influenced by thinking with islands as key sites in the 'Age of Entanglement' (Daou and Pérez-Ramos, 2016: 9), all highlight specific co-relational entanglements, and the living of life 'in the ruins' of modernity. It is in paying attention to these patchworks of disturbances and effects, and the pragmatic actions put in place at a particular time and place, that, for such approaches, 'we wander without becoming lost' (Glissant, 1997: 203). ${ }^{11}$ Thus, we examine how drawing upon and working with islands in these debates and developments in critique, art and activism brings to the fore the figurations and co-shaping of relations, emergent disturbances and effects, which we characterise as Patchwork ontologies.

\section{Onto-epistemology}

After establishing that engaging islands and islanders in the Anthropocene is seen as productive for the generation of relational ontologies, the second half of the book turns to how islands have been worked with in the production of distinctive relational approaches to epistemology: those of onto-epistemology. In a relational ontology, questions of epistemology are not entirely separate from those of ontology, but are 'onto-epistemological': in other words, knowing is not a product of passive reflection but inextricable from being itself. In Chapters 4 and 5 we suggest that 
Anthropocene thinking with islands about epistemology can be approached via two lenses or tendencies, demarcating two extremes of a sliding scale or continuum in which forms of relation become increasingly strange or weird from a modernist perspective: Correlation and Storiation.

Just as island relational ontologies could be grasped on a disruptive continuum, captured at either pole through the lenses of Resilience or of Patchworks, depending upon whether we are in or beyond modernity, understood as a grid of fixed space and time, the same is the case when it comes to how we think with islands onto-epistemologically. In terms of the heuristic schema of onto-epistemology, the analytical approach of Correlation, like the ontological approach of Resilience, marks one end of the continuum. Correlation, while moving away from linear causal understandings, nevertheless maintains a modernist Newtonian conception of linear time and flat Euclidean space. At the other end of the continuum, Storiation, much like Patchwork ontologies, radically disrupts flat or modern notions of space and time. As we have emphasised above, for us this distinction is absolutely key for grappling with the changing ways in which Anthropocene thinking draws upon and works with islands to increasingly displace and erase modernist categories of thought; bringing to the forefront the non-modern implications of relational entanglements and feedbacks.

Chapter 4 analyses how Correlation approaches to ontoepistemology maintain a knowing human subject and a world of patterned regularity amenable to policy intervention. Here, the island emerges as a 'correlational technology' where island changes are often seen as the first signs or indicators of the looming dangers of climate change (Watts, 2018; Baldacchino, 2020). As Elizabeth DeLoughrey (2019: 166) says, 'the island is understood not just as the Earth, but as its anticipated future' (Fitzpatrick and Erlandson, 2018; Watts, 2018; Larjosto, 2020). Central for us, however, is not the fact that islands are vulnerable or exposed but rather the logic at play in seeing or perceiving something through changes in another entity: an (onto)epistemology of inter-relation 
and correlation rather than one of linear cause-and-effect. In these approaches it is not so much that climate change 'causes' island changes but more that it manifests as or, in fact, is these changes. In onto-epistemological framings we move from a temporal and spatial line of movement to one of synchronicity - which enables humans to better register, read and sense transforming planetary conditions through their real-time effects. In Chapter 4 we explore how drawing upon and working with islands has become widely understood to be generative of Correlational approaches. The now ubiquitous trope, of islands as the 'canary in the coalmine' for climate change, dramatically illustrates how Correlation is one of the most prevalent framings for grasping the meaning and impact of planetary change in contemporary academic and policy-making literatures.

By contrast, approaches of Storiation, examined in Chapter 5, forcefully disrupt the modern binaries of subject/object, thought/ being - and thus move beyond Correlation's focus upon interrelation - problematising the way that modern thought maintains the separation of entities in time and space. Instead, Storiation onto-epistemologies engage islands and island cultures as significant 'holding' sites, not generating knowledge of relations but highlighting the disruptive potential of the e/affects, traces and afterlives of actions and events by way of speculative approaches and practices. Thus, Storiation has an explicit concern for the ways in which the ongoing legacies (for example, of modernity and colonialism) are occluded by way of Correlational frameworks which attempt to modulate around the status quo. Instead, Storiation approaches work with islands as holding the marks and signs of effects in other ways; registering the impacts of actions in 'weird', 'ghostly', 'haunting', and 'quantum' ways (as just some examples we discuss: Morton, 2016a; Sharpe, 2016; Wolfe, 2017; Mathews, 2017; Barad, 2019; King, 2019; Neimanis, 2019; Farrier, 2019, 2020; Wang, 2020; Clark and Szerszynski, 2021; Perez, forthcoming).

The power of thinking with islands and island cultures in the development of Storiation approaches has involved a significant 
turn to, and particular readings of, certain island writers. We consider how the island scholar and poet Kamau Brathwaite (1981, 1993, 1999) can be seen as opening up the line of thought of Storiation; precisely because he understands the island 'tidalectically' as the embodied, intra- (rather than inter-) relational movement associated with the ongoing legacies and effects of colonialism which are held in and hold the present. In the contemporary era we find Brathwaite's way of working with islands to be highly influential and updated in such prominent works as Christina Sharpe's In the Wake (2016) and Tiffany Lethabo King's (2019) The Black Shoals: Offshore Formations of Black and Native Studies. Both reflect a strong turn to draw upon the works of certain island writers, like Brathwaite, who engage land and water simultaneously, as a vitally important pathway, or holding space, for registering Black or Indigenous subjectivities and resistances - speculatively reading the ongoing legacies, effects and hauntings of colonialism which problematise separations of the present from the past.

Both the (onto)epistemological analytics of Correlation and Storiation are therefore situated and relational, and can also be understood as non-modern or non-anthropocentric approaches to material or contextual capacities of knowing. Where they differ is that in Correlation approaches it is the patterned regularity of inter-relational effects which is key, as adaptive interactive life co-relates in ways which are amenable to facilitating human understanding and prediction, enabling the reading of environmental change. In Correlation, relational interaction thus takes place in a universal or 'one world' modernist conception of time and space, and Correlational practices are seen as replicable models which can be widely applied and exported elsewhere, so that the rest of the world can learn from islands and islanders. In Storiation, by contrast, relational interaction much more explicitly problematises these modern notions of linear time and flat space. Thus, Storiation approaches often speak of strange, unexpected or irreducible forms of intra-action associated with the afterlives, legacies or ongoing effects of such forces as consumerism, waste production, colonialism and capitalism (Alaimo, 
2014; Morton, 2016a; Mathews, 2017; Wolfe, 2017; Farrier, 2019; Barad, 2019).

In Storiation, islands and island cultures are regularly employed to highlight how there is no 'away' and no 'past' in the Anthropocene (Morton, 2013; Ghosh, 2016; Cyphers, 2019). This is exemplified by how, when it comes to such vast, multidimensional forces as global warming, far from being isolated or cut off, islands hold the traces and legacies of processes which are often more difficult to detect from mainland positions and perspectives. Thus the island is increasingly figured as not only existing within complex relations of coloniality and global warming but as holding these forces and being held by them, disrupting hegemonic, modernist scales and distinctions (Sharpe, 2016; Yountae, 2016; King, 2019; DeLoughrey and Flores, 2020). The marked rise in the importance of, what we call, 'Storiation' for contemporary thinking, articulates the engagement with the geographical form of the island and island cultures through more speculative methods, which highlight forces and intensities which modernist methodologies too often fail to capture.

\section{Correlation - Chapter 4}

This chapter turns to the powerful ways in which Anthropocene scholarship and policymaking focuses upon islands to generate ways of knowing through Correlation. In this approach, islands are framed as enabling a different form of knowledge, derived from correlation rather than causation; where the registrations or effects generated by the interactive life of islands on many different scales is understood to provide new insights about the world. Thus, for example, we can understand islands as bearing the relational effects of their interactive becoming within global warming, rising sea levels, nuclear fallout, and other forces associated with the Anthropocene (Intergovernmental Panel on Climate Change, 2007; Nwanze and Sinon, 2013; Hall and Sanders, 2015; Cole et al, 2016; Fitzpatrick and Erlandson, 2018). Islands therefore enable ways of understanding climate impacts on ecologies and become significant sites for understanding based upon new technologies 
of dating and testing for different chemical and organic traces (Springer et al, 2017). In this way, islands and island beings are held to 'speak to us', and when they do they tell material stories of life's inter-relation and interdependencies in the Anthropocene.

In modernist approaches to knowledge, the search for universal laws of causation is seen as essential to control and command the non-human world, extracting resources and developing 'Man' as distinct from the world of 'Nature'. This way of thinking about knowledge emphasises the distinctions key to modernist epistemology, the binaries of thought and matter and of human and world, constructing a hierarchy of understanding of a universal or 'one world' world. But working with islands after the end of such a world enables other insights. Here, Correlational thinking aligns with that of Resilience ontologies in challenging modernist assumptions; focusing not on entities held to have essences, but on relational interactions, establishing regularities, patterns or habits reiterated across and through systemic interactions.

Correlational knowledge is the knowledge of experience, of practice, of habit, often embedded in relations and embodied in modes of being and working. It is the mode of interactive becoming of life and it leaves its traces upon the flesh of the world, in the evolution of species and landscapes. For example, correlational insights enable us to spot the likely occupation of workers and labourers through their muscle distribution, their bodily gait, times and routes of travelling, areas of habitation, and so forth as Michel Serres (2011) argues, the seaman becomes one with his ship and brings the sea home with him in his body (see also Ingold, 2015). In the same way, the snout and tongue of the giant anteater, through patterns of correlational dependency on the food source of ants, tells us much about the nature of anthills (Kohn, 2013). These patterned relations of iterative interaction enable us to learn through correlation. As Thom van Dooren (2014: 27, emphasis in original) clarifies, species can be understood to correlate or to register environmental effects:

... a species must be understood as something like a line of movement' through evolutionary time. But it is much more than an 
empty trajectory. Each species embodies a particular way of life... an ongoing intergenerational process of becoming - of adaptation and transformation - in which individual organisms are not so much 'members' of a class or a kind, but 'participants' in an ongoing and evolving way of life.

The key point is that species register their relations in their ways of becoming. For example, species co-evolve over time, like the bee orchid Ophrys apifera which co-evolved to mimic a particular species of bee, now extinct. Species can therefore 'speak to us' about changing environmental relationships and conditions, holding traces and registrations of relations and of entities which can no longer be observed directly. Correlation is always indirect, always a measure or register of a relation and a way of tracking changes in relation. The evolution of species thus becomes a powerful way of registering the interactive effects of human-induced climate change, pollutants, nuclear testing, and transforming planetary conditions; so that 'evolutionary biology can inform governance and policies in the Anthropocene' (Jørgensen et al, 2019: 527).

As noted, islands and islanders are widely understood to have the sensitivities and affordances necessary to reveal and register processes of anthropogenic influence which would otherwise go unseen by the wider world (Benwell, 2011; Hanna and McIver, 2014; Walshe and Stancioff, 2018). For international committees, managers and policymakers, islands are harbingers or advanced indicators of what is to come elsewhere; from the fires which engulfed Australia in 2019 and 2020, to the sinking islands of Tuvalu, Anote's Ark, and the loss of island species around the world. As Watts (2018: 149) says:

Islands are often on the planetary frontline of environmental change. Their long shore-lines and specialized ecosystems are finely tuned and sensitive places, barometers for the Earth ... the litmus test for the urban future.

Long held as key sites for understanding relational entanglements and feedback effects in evolutionary theory, biology, anthropology, 
geophysics, and many other disciplines, islands and islanders are notable Correlational modes for sensing and revealing the forces of global warming, rising sea levels, nuclear fallout, intensified hurricanes, and a whole range of other shifting planetary conditions (Cantieri, 2017; Cass, 2018; Pugh and Chandler, 2020b; Grydehøj and Kelman, 2020; West, n.d.). In saying this, therefore, we are not only pointing to how islands are reinterpellated as a 'living laboratory' (Watts, 2018: 105), in the sense of being small and confined sites for modernist methodologies of investigative research (Grydehøj and Kelman, 2017). We argue that the island, and island life itself, is widely seen as enabling the generation of onto-epistemologies operating on different, correlative rather than causal, assumptions in order to stimulate alternative frameworks of knowledge and knowing to address the challenges of the Anthropocene.

Along with island ecologies, probably the most high-profile illustration of this in contemporary thinking is the widespread celebration of Indigenous islanders' own correlational abilities (Salick and Ross, 2009; Breckwoldt and Seidel, 2012; Enn, 2015; Camus, 2018; Suliman et al, 2019) ${ }^{12}$ seen as a vital attribute for survival in the Anthropocene (Percival, 2008; De Souza et al, 2015; Forest Peoples Programme, 2019). As First Peoples Worldwide (n.d.) say, 'Indigenous science and knowledge are based largely on bioindicators, or natural signs ... Learning from nature in this way is an integral part of the Indigenous worldview that all things are connected, and that nature, when respected, can be a benevolent part of the whole community'. Indigenous islanders are often characterised as possessing unique correlating and sensing expertise, lost to the Moderns:

On these small atolls the ocean and its rhythms, the endless sound of the waves breaking on the reef, and the tides, constantly contracting and expanding around the islands like a heartbeat, feature in most aspects of daily life. Navigational skills have allowed a handful of people from these islands to align themselves in this ocean world and to predict sailing and weather conditions. Navigators have interpreted the formation and colour of clouds to 
identify islands over the horizon. Birds and certain species of fish would give an indication of the distance to land. Star paths were followed when travelling greater distances. Most impressively, ocean swells, reflected from far away islands and reefs, would echo through the canoe and its navigator, and would be recognised like the face of an old friend. (Robertson, 2018: 50-51)

Thus, Indigenous islander correlational practices are increasingly considered to be extremely useful in the 'forecasting of extreme weather conditions' (Siutaia, 2020). In such approaches, living and evolving knowledge of relational interaction is often understood as (or previously relegated to) 'Indigenous knowledge'. However, as we explore, Correlational approaches have received a high-tech boost and makeover in the Anthropocene, taking an algorithmic form of 'if this ... then that', associated with contemporary forms of computation involving Big Data and the Internet of Things (Chandler, 2018b). Thus, in Chapter 4 we illustrate the prevalence of Correlational logics in a wide range of practices, where working with islands is widely understood as significant to the generation of new approaches, highlighted by the trope of the 'smart island'. Here, the prolific use of Big Data combined with extensive networks of sensors enables rapid policy responsiveness to changing island coastlines and rising sea levels (United Nations Climate Change, 2019); the remote sensing of coral bleaching around islands acts as a bio-sensor of environmental change (Foo and Asner, 2019); and there is growing interest in algorithmic correlation with social media feeds to see emerging island disasters (Cavallo, 2017; Whyte, 2017). Through such examples, we demonstrate how working with islands as key sites for understanding relational entanglements and feedbacks enables novel approaches, and plays an important role in the generation of Correlational onto-epistemologies in contemporary thinking.

\section{Storiation - Chapter 5}

Correlation approaches, in our framing, work to establish island onto-epistemologies as important to survival in the Anthropo- 
cene. They generate forms of knowing that are capable of grasping entities as having attributes and affordances in relation, rather than possessing fixed and distinct 'essences'. Correlation is dependent on regular, reiterated patterns of effects. Whilst different from the modern logics of causation, it therefore still operates to generate generalisable forms of calculation, measurement and comparison - like the construction of temperature via correlative means (the expansion of mercury in a glass tube when heated). In Chapter 5, we seek to highlight and give shape to an approach at the other end of the sliding scale of relational onto-epistemologies that frequently uses island experiences to generate a more disruptive form of onto-epistemology: which we are conceptualising as Storiation. Central to Storiation is registering the ongoing afterlives, traces, hauntings and effects of such significant forces as colonialism, modernity, global warming, nuclear radiation, rising sea levels, and waste production; where islands and island cultures regularly emerge as important sites for investigation and island writers have increasingly come to the fore (Brathwaite, 1999; Teaiwa, 2011; 2012; Sharpe, 2016; Morton, 2016a; Yountae, 2016; DeLoughrey, 2019; Salt, 2017; Theobold, 2018; Jetñil-Kijiner, 2019; King, 2019; Perez, 2020b, forthcoming; Clark and Szerszynski, 2021).

What distinguishes the Storiation analytic is the holding together of entities and effects, registered through islands and islander lives, in ways that deeply problematise modernist framings of the spatial and temporal locations of objects and events (Alaimo, 2014; 2016; Morton, 2016a; da Silva, 2016; 2017; Farrier, 2019; Neimanis, 2019; Wang, 2020). Storiation approaches engage islands and island cultures to speculatively bring to the forefront of thought intra-actions and effects (rather than coherently graspable interrelations) through their afterlives, hauntings, and their ongoing and transformative traces (Brathwaite, 1999; Sharpe, 2016; Mathews, 2017; Theobold, 2018; King, 2019; Barad, 2019; Clark and Szerszynski, 2021). Key to the analytic of Storiation then is how this onto-epistemological approach seeks to speculate through the island, islanders' embodied movements and their practices, rather than critically stand back, in the way of modern reasoning 
or Cartesian 'Man', and tell stories about them (Brathwaite, 1999; Wolfe, 2017; Sharpe, 2016; King, 2019; Clark and Szerszynski, 2021). In this way, to think in Correlation and Resilience terms of entities adapting to others via feedback, or to pose the problematic in terms of Bateson's cybernetic framing of 'organism plus environment', still separating entities and communication or thought and being, differs from the articulation of Storiation as an embodied and material onto-epistemology of intra-relation. Vicky Kirby's (2011: xi) work has been very helpful in enabling us to think through the analytics of Storiation, in her view that 'interactive' life should be understood as textual as 'life reads and writes itself', in ways which foreground how the languages of feedback effects fail to capture how entities do not pre-exist feedback effects or communication but are constituted with them (see also da Silva, 2016, 2017; Barad, 2019).

We can start to draw out some of these key aspects of Storiation by turning to the work of Timothy Morton (2013: 36), who argues that in the Anthropocene there is no 'away' - what we do 'sticks' and objects and experiences can appear to us through their legacies and afterlives which we can read in their ongoing material effects. For Morton (2016a), some of these effects play out more immediately - such as the powerful hurricanes hitting islands around the world every year - while others stretch out for hundreds of thousands of years, e.g. the time it takes for carbon to dissolve in the oceans surrounding islands. Thus, in working with islands as important sites of relational entanglements we come to see a world which holds strange 'attractors' and interconnections, rather than one of clear separations, linear causality or a hierarchy of branching 'trees' (see also Alaimo, 2016; Hejnol, 2017). For authors like Morton (2016a), the effects of entangled relation mean that engaging islands can provide valuable insights into the 'afterlife' of objects and events in ways which transform modern understandings of them as isolated or contained. The (island) future then becomes entangled with the past as the 'afterlife' of relational effects continue to reverberate across time and space, as we detail in the chapter, in 'strange', 'weird' or 'quantum' ways (da Silva, 2017; Wolfe, 2017; Barad, 2019; Neimanis, 2019). 
Storiation is not merely a way of seeing and speculating about the effects of environmental damage; there is much of modernity that needs to be confronted through the ongoing effects that are constitutive of the present rather than part of the past. Christina Sharpe's In the Wake (2016) does precisely this in highlighting the ongoing effects of chattel slavery and its afterlives in the present:

These are questions of temporality, the longue durée, the residence and hold time of the wake. At stake, then is to stay in this wake time toward inhabiting a blackened consciousness that would rupture the structural silences produced and facilitated by, and that produce and facilitate, Black social and physical death. (Sharpe, 2016: 22)

Thus Storiation - the material effects or registrations of being in the world - troubles the separations of space and time of modernity unlike approaches of Correlation. It is through Storiation that islands and islander lives most powerfully enable the rewriting of modernity's attempts to construct a linear temporality in which the past and the future point in opposite directions. In the Anthropocene, whatever they say is 'over' or 'finished' is very much still with us.

Elizabeth DeLoughrey's Allegories of the Anthropocene (2019) emphasises how some feminist, ${ }^{13}$ postcolonial and Indigenous perspectives not only challenge the modern, mainland, 'god's eye' view of the island but also foreground how the narrative use of disjuncture and rupture 'demands a multiscalar method of telescoping between space (planet) and place (island)' (DeLoughrey 2019: 2). Disrupting linear histories of 'pasts' as 'events' separated from the present and, through islands and island cultures, DeLoughrey works at 'uncovering other (feminized) "roots" and agents' (2019: 25); 'telescoping' (2019: 2) together that which a modernist methodology seeks to exclude or to disavow. Understanding the island or islander as holding together entities and relations, causes and effects, in these ways, in the contemporary work we examine in the chapter, is a central aspect of what we describe as the analytic of Storiation (Teaiwa, 2011; Farrier, 2019). Thus, as a further illustration, for DeLoughrey (2019: 121), the sculptural work of 
someone like Tony Capellán, which almost exclusively comes from objects, such as plastics, washed up on the shores of Santo Domingo, Dominican Republic, is 'not a colonial archive but rather a site of witnessing, rendering the "secret" of wasted lives visible to the more privileged classes who benefit from the labor and the sacrifices made by the undifferentiated poor.'

It is important to clarify the stakes here. As in the case of the other analytics which we develop in this book, what we are doing with Storiation is drawing out a cross-cutting, broader analytic; which, we think, is highly prevalent across a range of contemporary works that increasingly engage islands for the generation of Anthropocene thinking. Thus, to be clear, we are not saying that the entire body of work of authors like DeLoughrey, Sharpe or Morton is Storiation (just as Glissant's entire body of work could not be reduced to Patchworks), but that there are disernable patterns or prominent lines of thought which can be analytically highlighted across them as representative of an important contemporary register, or prevalent logic, in Anthropocene thinking. We are also therefore not seeking to speak for or on behalf of someone's body of work, but rather drawing out certain elements which can be mapped into the emergence of these broader analytics. As implied above, the Storiation chapter explores how certain approaches map across a wide range of works; as when Perez (forthcoming), an Indigenous Chamoru from Guåhan (Guam), says that 'much of' the Pacific ecological and climate change literature he is working on with Kathy Jetnil-Kijiner and Leora Kava 'expresses Storiation, or the afterlives and haunting legacies of imperialism in the Pacific'. In turn, even as terms like 'haunting' may at first sight appear to denote a fairly Western/Judaeo-Christian concept, further drawing upon Katerina Teiawa (2011, 2012; 2015), Nigel Clark and Bronislaw Szerszynski (2021), Mimi Sheller (2020), Emanuela Borgnino (2020) and Tamara Searle (2019), we variously examine their Storiations of Indigenous spiritual practices, from shamanistic and African-rooted traditions such as spirits coming into people's bodies, through dance, music and trance (all of which have strong histories of island-practice). 
We attend to how certain island and oceanic tropes, and strands of island scholarship, are being explicated in works within contemporary Indigenous and Black Studies, often at the forefront of onto-epistemological approaches of Storiation (see, for example, Moten, 2003; Sharpe, 2016; Yountae, 2016; Hessler, 2018; Neimanis, 2019; King, 2019; DeLoughrey, 2019; Wang, 2020). Of particular importance for the analytics of Storiation is the work of the Barbadian historian and poet Kamau Brathwaite. Sharpe's (2016: 177) In the Wake explicitly foregrounds how Brathwaite prefigures her own approach to registering how Black life embodies, intra-relationally, the legacies of colonialism; stating that Brathwaite's way of Storiating Caribbean life is Black being in the wake'. Similarly, Brathwaite is also central to King's (2019) The Black Shoals: Offshore Formations of Black and Native Studies. Brathwaite's (1999) onto-epistemology of 'tidalectics' not only profoundly disrupts mainland, continental and modern frameworks of space-time, and binaries of human/nature, it shows how Caribbean islanders emerge, literally as new forms of life, in the wake of colonialism:

Why is our psychology not dialectical - successfully dialectical - in the way that Western philosophy has assumed people's lives should be, but tidalectic, like our grandmother's - our nanna's - action, like the movement of the ocean she's walking on, coming from one continent/ continuum, touching another, and then receding ('reading') from the island(s) into the perhaps creative chaos of the(ir) future ... (Brathwaite, 1999: 34; italics in original)

This helps us to illustrate how Storiation speaks of intra-action and the holding together of dynamic forces and attractions, not a modern focus upon inter-action between pre-defined and separate entities. Brathwaite's 'nanna' will surely at times stand back and critically reflect upon the conditions of colonialism, but the key point for Brathwaite's onto-epistemology is that it is her daily routines and embodied movements themselves which are the dynamic forces holding in - living on in and maintaining the legacies of - the wake of colonialism. There is no critical 
separation, binaries, or linear understanding; the situation is one of a dynamic holding together of hauntings and traces 'receding ("reading") from the island(s) into the perhaps creative chaos of the(ir) future ....' (Brathwaite, 1999: 34). This comes out particularly well in the tropes which Brathwaite employs to characterise colonialism on islands. 'Tidalectics' thus speaks of how the focus upon intra-action deeply problematises modern notions of separate entities, predictive time, and flat space, instead favouring a speculative process of thought that decentres the notion of the modern subject, starting from islander and island materiality.

For Sharpe (2016) and King (2019: 207), Brathwaite is a key figure for understanding how Black life lives on in the wake of slavery and colonialism, with his 'old woman of Caribbean history engaged in the morning ritual of sweeping who walked on the water with sand in her toes'. For King (2019: 207), Brathwaite's focus upon the embodied movements of the old woman disrupts the simplicities of inter-relational and modern frameworks of reasoning - human/nature, mind/body, land/water divides - and reflects how 'Land is not the traditional element used to analogise Black flux or think about dynamic, fluid, and ever moving Black diasporic subjectivity' (see also Wang, 2020). As we detail in the chapter, in her own Storiations of Black and Indigenous life, King (2019: 29) employs such methods as 'critical fabulation' and 'speculative bricolage' in order to effectively hold together the traces, hauntings, ghosts and afterlives of colonialism which are embodied and constitutive of the present. But here we can already see how such contemporary scholars are profoundly influenced by island writers and poets, such as Brathwaite, who have long performed in ways which 'can be turned back against continents... offering a model of how to live complexly rather than through the simplifications and essentialisms that have characteristically been projected onto islands' (Edmond and Smith, 2003: 12).

The figure of the island and these strands of island scholarship have been important for the development of the Storiation analytic which characterises an increasing range of contemporary 
thinking. We examine, in addition to those noted, related works including those by Cary Wolfe (2017), Andrew Mathews (2017), Karen Barad (2019), David Farrier (2019, 2020), Jackie Wang (2020), Claire Colebrook $(2016,2019)$, and the concluding chapters of Glissant's (1997) Poetics of Relation. In Storiation approaches, islands and islanders are understood as intensive sites, holding and registering the hauntings and traces of relations, that do not cut the past from the present. Islands and islanders, engaged as these worlds of legacies and effects, of the dynamism of embodied intra-active becoming, rather than inter-action, are seen to offer alternatives to Correlational approaches: Storiations of the differentiating powers of colonialism, of the emergence of tidalectic psychologies living on in the wake, of island dances, vodou and shamanistic practices, of species long extinct, of the consumerisms that haunt islands in strange ways - Storiations of how there is no 'away' and no past in the Anthropocene (Morton, 2013; Ghosh, 2016: 26).

\section{The Importance of Island Studies in the Anthropocene}

There is little doubt that the widespread contemporary interest in islands mirrors the rise of non-modern, relational, non-linear and more-than-human thinking across many academic disciplines and policy practices. But, as explored in this introductory chapter, this book makes the argument that the engagement with islands in many debates today is not merely caught up in the slipstream of contemporary social and philosophical trends, but is important to the ontological and onto-epistemological framing and tools with which the new epoch of the Anthropocene is being grasped. What we therefore undertake in this book is an analysis of the 'work' that thinking with islands, island imaginaries, island writers, artists, poets, activists, and island problematics is doing in these debates. This is because we believe that we can more fully understand why and how Anthropocene thinking is as it is today if we are able to open up questions of how working with islands is playing an important and generative role. 
Not only thinking about, but with islands (Gillis, 2004) has become an important resource for alternative and non-modern relational ontologies and understandings in the Anthropocene. We suggest that there is a need to not only critically focus upon how the modern episteme reductively grasps islands (to be clear, this is still important), but to also establish a new critical research agenda focused upon how islands are being enrolled in debates about the Anthropocene as key sites for understanding relational entanglements, in the generation of many different forms of relational ontology and ways of knowing. Central here, as we want to stress, is how working with islands or relational thought per se is not one homogenous 'other' to modernist or mainland approaches, and so it is important to start a new conversation about how we engage in working through the rich variety of possibilities and opportunities that these approaches afford. It is the shift to engaging and working with islands in wider Anthropocene scholarship, policymaking, art and activism, which we believe points towards some important stakes for a critical agenda going forward. This would expand analysis concerning the question of why and how engaging islands has been so productive and generative for Anthropocene thinking. Thus, in the concluding chapter (Chapter 6), we elaborate upon how we see this book as an initial opening for a new critical agenda for island studies in the Anthropocene. But before that, in the intervening chapters, we will be laying out our proposed heuristic set of analytics for working with islands in the Anthropocene - the relational ontologies of Resilience and Patchworks, and the relational onto-epistemologies of Correlation and Storiation.

\section{Notes}

${ }^{1}$ Whether researching creolisation in the Caribbean (Brathwaite, 1981, 1993; Glissant, 1997), the migration of peoples in Oceania (Hau'ofa, 2008; Rakuita, 2017), the dynamism of shifting or disappearing ice-sheet islands (Riquet, 2016; Steinberg and Kristoffersen, 2017), the Silk Road archipelago (Xie et al, 2020), or the construction 
of new human-made islands (Jetñil-Kijiner, 2019; Dodds and della Dora, 2018; Bonnett, 2020), what we have elsewhere called these 'relational' and 'archipelagic' turns in island studies (Pugh, 2013, 2016a, 2018) have, over the past few decades, radically decentred the notion of the isolated and static 'island' to instead emphasise mobile, multiple and interconnected relational forms. It should not be underestimated just how much the relational and archipelagic turns have expontentially developed (Baldacchino, 2019). This is illustrated by Bongie's (1998) Islands and Exiles, DeLoughrey's (2007) Routes and Roots, Thompson's (2010) Imperial Archipelago, Joseph's (2019) Sea Log: Indian Ocean to New York, and Martínez-San Miguel's (2014) Coloniality of Diasporas, as examples which focus upon colonial relations; Stratford et al's (2011) foregrounding of the archipelagos rather than islands of the world; Suwa's (2007) and Hayward's (2012a, $2012 \mathrm{~b}$ ) development of the 'aquapelago'; Ingersoll's (2016) Waves of Knowing which offers a 'seascape epistemology'; Louis and Kahele's (2020) invocation of Kanaka Hawai'i Cartography; Hessler's (2018) various engagements with Brathwaite's 'tidalectics'; Rankin (2016), Pugh (2016a) and Pugh and Grove's (2017) focus upon 'archipelagic assemblages'; Sheller's $(2000,2007)$ work on archipelagic 'mobilities'; Dening (2007), King (2007) and Connell's (2018) work on migration and islanders; Crane and Fletcher's (2017) focus upon archipelagic thinking in island literatures; Loughran's (2019) on archipelagic education; and Roberts and Stephens (2017) foregrounding of the archipelagic nature of the Americas. There are many, many others besides who foreground islands as relational spaces (Stratford, 2003; Steinberg, 2005; Hay, 2006, 2013; Papoutsaki and Harris, 2008; Clark and Tsai, 2009; Baldacchino and Royle, 2010; Alexander, 2016; Joseph, 2013; Starc and Stubbs, 2014; Ronström, 2015; Benítez-Rojo, 2016; Kearns and Collins, 2016; Bremner, 2016; Hong, 2017; Graziadei et al, 2017; Murray, 2018; Vale, 2018; Evans and Harris, 2018; Carter, 2018; Nimführ and Sesay, 2019; Davis, 2020; Isaacs, 2020). For an excellent overview of a variety of recent approaches see Michelle Stephens and Yolanda Martínez-San Miguel's (2020) collection Contemporary Archipelagic Thinking.

${ }^{2}$ We are not the first to use the term 'Anthropocene Islands'. Given the vast amount of contemporary work on islands in the Anthropocene it is not surprising that the term has been employed in a number of projects and works, each operationalising it in their own specific ways to think through islands as sites for Anthropocene thinking. So 
far as we are aware, the term has been used in at least three quite distinct ways, prior to and alongside its use in our work. We believe that it was perhaps first used in September 2017 with the launch of the exhibition 'Anthropocene Island' at the Tallinn Architecture Biennale (ecoLogicStudio, 2017a; 2017b): an impressive set of designs involving scientists, social scientists, artists, and many others - for what an island might look like in the Anthropocene (we discuss this example in detail in Chapter 2). Another example of how the term 'Anthropocene Islands' has been employed is by Peggy Cyphers and others (2019) in the exhibition 'Anthropocene Island: Colonization, Native Species and Invaders'. This uses the term in order to register the ongoing legacies and hauntings of capitalist consumerism, specifically plastic, and how islands amplify and illustrate how there is therefore no 'away' in the Anthropocene (see also Drifters Project, 2019). We discuss Cyphers et al's (2019) work in Chapter 5. A third usage of 'Anthropocene Islands' is employed in Amelia Moore's (2019a) Destination Anthropocene: Science and Tourism in the Bahamas. Moore (2019a: 5) defines her approach, which develops anthropology in the Anthropocene, as focusing upon 'the discursive and practical entanglement of science and tourism, which I call "Anthropocene Islands". Based upon ethnographic fieldwork into science, tourism and the Bahamas, Moore draws attention to how islands are key sites for examining contingent relations between class, race, capital accumulation, exploitation, and other forces, as these manifest and are expressed in global environmental change (see also Moore, 2015a, 2015b, 2016, and 2019b).

3 Joseph (2020: 193) reflects the view of many contemporary authors when she positively foregrounds how the liminality of islands [remains] 'outside the frameworks of mainland narratives' (see also Gómez-Barris and Joseph, 2019).

${ }^{4}$ Here we suggest that scholarship might be further interested in exploring how, or indeed whether, approaches to Anthropocene scholarship in China, India and other large continental mainlands, are changing through how they draw upon and engage islands. Whilst beyond the remit of this book, we think this would be a very important area to develop in the future, and would be particularly interested in speaking to anyone who is engaged in this area of research.

${ }^{5}$ We capitalise the terms 'Resilience', 'Patchworks', 'Correlation' and 'Storiation' when we deploy them heuristically, as the key analytic categories developed in this book. 
${ }^{6}$ Thanks to Stephanie Wakefield for drawing our attention to this interview material.

${ }^{7}$ For example, when Anna Tsing, Andrew Mathews and Nils Bubandt (2019: 186) produced a special edition for Current Anthropology on what they called the 'Patchy Anthropocene' - 'a conceptual tool for noticing landscape structure' - a third of the articles in that special edition, including Hadfield and Haraway's (2019) famous 'Tree Snail Manifesto' developed from work with Pacific Island tree snails, were derived from work on islands.

${ }^{8}$ The terms 'patchy' and 'patchworks' more generally seem increasingly prevalent in contemporary debates about anthropology and the Anthropocene in particular (see, for example, Tsing et al, 2019; Günel et al, 2020; Sheller, 2020). They align more generally with the rise of concern for how we are already in the Anthropocene and/or a general focus upon assemblages, knots of relations and co-entanglements. As we have discussed elsewhere (Chandler and Pugh, forthcoming, a), although there are overlaps with these developments, for us 'Patchworks' means something quite specific, as we examine in detail in this book.

${ }^{9}$ We thank Godfrey Baldacchino for this important observation about islanding becoming a 'verb' in Patchwork ontologies. This is something we develop in Chapter 3.

${ }^{10}$ Glissant (1997) capitalises 'Relation'. We will therefore also do so when explicitly referring to his work.

${ }^{11}$ We wish to emphasise that our point here is not that contemporary scholars necessarily cite an island scholar like Glissant (although, of course, many do; see, for example, Yountae, 2016; Last, 2017; Mentz, 2017; Yusoff, 2018, DeLoughrey, 2019; Colebrook, 2019). Rather, our key argument is that thinking with islands in Glissant's (1997) Poetics of Relation was an early exemplar for the Patchwork ontologies, being generated in Anthropocene scholarship today. It matters that Glissant's approach initially focuses upon islands and then was expanded outwards. Patchwork ontologies focus upon patchwork islands of refiguration forming in nodes or knots of assemblages across time and space, disrupting modern notions of flat spacetime which still hold in ontologies of Resilience. In Patchworks approaches, in this particular way, islands become the ontology of the world.

12 There is no clear definition of 'Indigenous knowledge'. This is not surprising considering that there are over 7,000 different Indigenous 
languages and peoples inhabiting extremely diverse environments. What we focus upon in this book is the various ways in which Indigenous islanders are understood to contribute to different ways of 'being' (ontology) and 'knowing' (onto-epistemology) from Moderns in debates about the Anthropocene.

${ }^{13}$ Explicitly feminist approaches to island studies scholarship which operate in these ways have also been developed by many other researchers, including, most recently, Karides (2016, 2017), Lama (2018), Coss (2020), and in the collection Gender and Island Communities, edited by Gaini and Nielsen (2020). 



\section{Resilience: The Power of Interactive Life}

\section{Introduction}

In the introductory chapter we analysed how Anthropocene thinking draws upon and thinks with islands as key figures for engaging the central concerns of relational entanglements, awareness and feedback effects. Thinking with islands stands in direct opposition to the homogenising and universalising approaches of 'mainland' modernity. In this chapter, we focus upon the sphere through which island approaches have most prominently entered mainstream debates about the Anthropocene: Resilience. Our key argument is that Resilience reflects a paradigm shift towards a relational ontology which centres upon the immanent interactive potentialities of life itself - an approach which is not merely illustrated by island life, but which, as we examine, is analytically derived from particular ways of engaging and thinking with islands. In Resilience, the world is beyond our powers to command and control in the way of modern reasoning. Instead, the immanent potentialities and processual becomings of (island) life itself becomes a selforganising problem-solver, bringing about adaptation and order out of chaos. For Resilience ontologies, interactive (island) life is understood as becoming more efficient and harmonious, rather

\section{How to cite this book chapter:}

Pugh, J. and Chandler, D. 2021. Anthropocene Islands: Entangled Worlds.

Pp. 41-67. London: Ubiquity Press. DOI: https://doi.org/10.16997

/book52.b. License: CC-BY 4.0 
than entropic and disordered, thereby articulating an alternative or immanent telos of development and change. Here, islands and island cultures have become important symbols of hope in debates about the Anthropocene for the wider world to learn from, and to give Moderns a second chance to learn how to adapt to and even to gain from, the forces of planetary change.

The first section of the chapter highlights the importance of understanding Resilience as a relational ontology which challenges the universal assumptions of linear causality and technological progress which underpin modernist policy approaches to governance. The second section draws out the analytical content of this ontology, focusing upon the most widely understood and discussed facets of island life - the powers of diversity, differentiation and interaction, and the key assumptions informing them: of interdependency and feedback effects. The concluding section turns to how some contemporary approaches are developing or extending this immanent island relational ontology to the more quotidian or 'everyday' interaction of what we call 'Patchwork' island ontologies, which are then taken up and examined in more detail in Chapter 3.

\section{Resilience as an Ontology of Adaptation}

Resilience ontologies, as deployed in contemporary governance discourses, mark a major shift from earlier, modernist constructions of environmental and resource care, particularly those of 'sustainability' (O'Brien, 2017; ' Wakefield, 2020). Prior to the move towards the problematic of relational entanglements, awareness and feedback effects, which today dominates Anthropocene thinking, concerns for environmental care were discursively framed in more top-down and managerial ways (Chandler and Pugh, 2020a; Wakefield, 2020). These were focused upon stability and equilibrium; in attempts, for example, to balance competing concerns, to produce with greater efficiency, or develop new materials or techniques (Derissen et al, 2011; Rist et al, 2014). In these older framings, there was a fixed set of assumptions about 
relations and therefore a greater confidence in an external perspective for managing or predicting changes in resource consumption and use. Here, islands were frequently constructed as being marginal and on the periphery of international debate, and often in need of saving by others (Farbotko, 2010; Cameron, 2011; Proctor, 2013; DeLoughrey, 2019). As we explore in this chapter, these older discourses of stability and constancy, and their concomitant assumptions of knowledge and control, highlight a 'mainland' approach which assumes a universal or 'one world' ontology of linear causality with fixed entity properties and law-bound relations. Resilience thinking in the Anthropocene works through the development of an alternative set of ontological assumptions about the world and its constitutive relationality; challenging the modern perspective and repositioning islands much more centrally within international debate.

Our focus in this chapter is how Resilience approaches utilise the science of relational feedbacks to generate solutions by drawing upon the dynamic powers of interactive life. Resilience is often defined as: 'the capacity of a system, community or society to resist or change in order that it may obtain an acceptable level of functioning and structure' (United Nations, 2004: Ch.1, S.1, 17). Or similarly, as 'the ability of groups or communities to cope with external stresses and disturbances as a result of social, political and environmental change' (Adger, 2000: 347). Such definitions reflect the central tenets of the seventeenth-century Latin word resilire: meaning the ability of physical materials to rebound and recoil (Reid, 2017), or 'leap back' (Gunderson et al, 2010: 64), into their original shape after the exertion of an external force. Whereas modernist framings of the world understand life to be composed of fixed entities, which possess discrete properties or essences, Resilience ontologies see life as a dynamic process in which entities are always in relation. As anthropologist and cybernetician Gregory Bateson (2000: 457) puts it: 'the unit of survival is a flexible-organism-in-its-environment'.

This focus upon the relational, processual or interactive ontology of life can be traced through a number of fields, from the biological 
science of evolutionary Darwinism to the physics of thermodynamics, and later interdisciplinary sciences of systemic interaction and cybernetics (Holland, 1998; Johnson, 2001; Harford, 2011). As we will discuss in the next section, the history of evolutionary biology has been particularly important in positioning islands as central for the generation and production of ontologies of Resilience. Both the theory of evolution and theories of thermodynamics suggest that 'life' can be cast as a struggle for order or system maintenance against the natural forces of entropy or decay (Darwin, 2010; Bateson, 2000). Thus, existence or continuity is not something that can be taken for granted but can be better grasped as processual change: as a product of iterative work and interaction. In other words, being is understood as a process of becoming or emergence. As Resilience theorists often comment, an 'equilibrium-focused view is attractive to humans', but 'it fails to capture the behavior of complex systems' (Gunderson et al, 2010: 230). Resilience theory is thus concerned with the 'nonlinear dynamics of complex adaptive systems' (Gunderson et al, 2010: 230; Svedin and Aniansson, 1987; Gunderson, 2001).

Rather than a linear unidirectional understanding of causality, where causal relations have already been set in motion and merely work themselves out across time and space (as in Newtonian physics), Resilience approaches see life as a product of interaction and therefore as less predictable and with more possibilities for alternative developmental pathways (Holling, 1973). They reject modernity's operating frameworks of command-and-control and instead focus upon the dynamic potentialities of interactive life. It is here that islands in particular are widely understood as extremely productive sites for understanding and developing Resilience, understood as a set of adaptive capacities. Islands are regularly framed as:

paradigmatic places of human-environment relationships. Island livelihoods have a long tradition of existing within spatial, ecological and ultimately social boundaries and are still often highly dependent on local resources and social cohesion. Island cultures and their rich biocultural knowledge can be an important basis 
for revitalizing and innovating sustainable human-nature relationships ... islands can serve as real-world laboratories. (Kueffer and Kinney, 2017: 311)2

For Resilience ontologies, the whole island system, including island ecosystems and cultures, will always be more than the sum of its parts (in contrast to reductionist, modern and atomised understandings of life) (Barnett, 20013; González et al, 2008). In this way, the feedback effects necessary for complex self-adaptive individuals, communities and systems to operate efficiently are said to enable adaptive transformative effects. It is the power unleashed by complex system relations that needs to be understood, accessed, redirected and repurposed. Thus, it is broadly understood that island cultures also exemplify the dynamic relational ontology of interactive life which is central to Resilience thinking:

Despite the rich cultural diversity across and among the subregions of Micronesia, Melanesia, and Polynesia, at the center of what we believe it means to be human is a shared life philosophy of balance, harmony, and deep connectedness. Our epistemologies, knowledge systems, and practices are premised on relational spaces - hermeneutical dimensions of life worth living... (Vaka'uta et al, 2018: 127)

For Resilience analytics, this relational knowledge and interactivity, exemplified by island ecosystems and island cultures, is a valuable resource (Nicks, 2017). Indeed, it has become central for research and practice concerned with unlocking and enabling the resilient potentialities of (island) life to emerge (Salick and Ross, 2009; Raygorodetsky, 2017). Resilience operates on these potentialities of island life and cultures, by drawing out how resilient capacities are part and parcel of a whole island socio-ecological system accessible to and used by islanders (Percival, 2008). In a world where it appears that the application of human science and technology to control or direct nature has undermined natural processes of regulation - including the catastrophic unintended consequences of climate change and global warming - Resilience 
seeks to slow down this runaway process by restoring more power to (island) life itself; seeking alternative ways forward that redistribute understandings of agency:

As a home to important flora and fauna, with rich cultural roots and heritage, island communities are often characterized by their deep social ties with the natural environment. However, due to environmental degradation, impacts from climate change including slow (e.g. sea level rise) and sudden (e.g. hurricanes) onset events and the associated changes to livelihood structures and opportunities, islands throughout the world face increasing threats. In order to understand and appropriately address livelihood risks in these communities and to identify opportunities for resilience-building, there is an urgent need to shed light on the historical and cultural context of island societies and ecosystems. These approaches should build upon local and traditional knowledge and be grounded in established practices developed by island communities over centuries which continue to be heavily impacted by current political and economic trends. (De Souza et al, 2015: 3)

For Resilience analytics, islands and island cultures are key to teaching the rest of the world how to adapt to transforming planetary conditions (De Souza et al, 2015; Kueffer and Kinney, 2017; DeWeerdt, 2019). Thinking with islands and island cultures is said to challenge understandings of a world framed in terms of a top-down modern telos of progress, and human/nature hierarchies, instead foregrounding a relational ontology of interactive life. Thus, for those who research regions which are dominated by island life, like the Pacific:

More significant than their exposure is the resilience of Pacific Islanders. The practices and knowledge associated with their resilience to environmental variability and unpredictability in the past suggest an adaptive capacity that is relevant to addressing the social-ecological effects of climate change now and in the future. Because of local limitations on resources and tight feedback loops, small island communities often see the limitations of 
their environment more readily, more quickly understand how anthropogenic and natural influences affect resource availability, and adapt accordingly. (McMillen et al, 2014: 1)

Once understood as secondary to modern, 'mainland' thinking, and even at times populated by 'savages' (Malinowski, 1921:1; Gillis, 2004, McMahon, 2016), in contemporary debates about the Anthropocene the situation is being reversed. It is increasingly islands and islanders which have become important sites of interactive life, interdependency and feedback effects and, thus, of resilient life:

Pacific islanders living on atolls are already negatively affected by climate change, facing threats to available fresh water and food. Yet the long-term familiarity with the variable nature of the atoll environment, where survival is held in a tight feedback loop with this unforgiving environment, has led to the development of adaptive and flexible resource management regimes which could provide a model for global responses to climate change. (Salick and Ross, 2009: 138)

Similarly, for Nakashima et al (2012: 11):

Small island societies have lived for generations with considerable and often sudden environmental change. The traditional knowledge and related practice with which small island societies have adapted to such change are of global relevance.

As Kelman and Randall (2019: 354) say, '[i] sland societies are often touted as being especially resilient'. Thus, today leading organisations, like Thompson-Reuters, regularly ask the question: 'How are small islands innovating to become more resilient in the face of growing pressures?' (Thompson Reuters Foundation News, n.d.). It is widely held that the rest of the world can learn a great deal from the answers (Rowling, 2017). The intensive focus is not confined to islands in the 'Global South'. Indeed, from the Dutch Wadden Island of Vlieland (Galle, 2017), to Tilos in Greece 
(Cordis, 2018) and Denis Island in the Seychelles (Nwanze and Sinon, 2013), islands have become arguably the figures of Resilience in the Anthropocene. From large-scale European Commission programmes like Clean Energy for All Europeans (European Union, 2019) to the recent success of the Netflix series 'Islands of the Future' (Filmproduktion and Arte G.E.I.E., 2016), there is a wide focus upon the Resilience capacities of islands and islanders from across the world. The running narrative of the five episodes of 'Islands of the Future', covering El Hierro, Orkney, Madeira, Iceland and the Danish island of Samsø, is that resilient technologies emerge on islands, more than elsewhere, because there is something essential to islands, at the level of ontology, which makes them particularly powerful candidates for resilience. For Gleb Raygorodetsky (2017: 264), in his book Archipelago of Hope, focusing upon islanders' own 'stories of resilience' gives us 'our best chance to remember - or learn - how to care for Earth in a way that keeps it healthy for our descendants' (Raygorodetsky, 2017: xix).

Today, in short, to foreground the vulnerabilities of islanders is increasingly said to 'downplay the resilience of communities, cast[ing] them as powerless ... [something which risks reifying] ... relationships of inequality between the powerful and weak through paternalistic interventions to "save" the powerless Other' (Mortreux and Barnett, 2009: 106). The relation between mainland and island has been reversed, with islands and islanders, significant sites for thinking through the relational interactions and resilient capacities of life itself, coming to the fore. 'Recent academic research has been increasingly moving beyond "doom and gloom" headlines to instead frame islands as sites of livelihood resilience to the impacts of climate change and disaster risk' (De Souza et al, 2015: 15).

\section{A New Ontology of Interactive Life}

Many readers may be familiar with the above types of analysis and approaches which foreground how important islands and island 
cultures are for the development of ontologies of Resilience. They may be aware that much of the very earliest Resilience theory was developed on islands (for examples, Gane, 1975; O'Keefe and Conway, 1977; Lewis, 1984; Campbell, 1984). But we think it is important to understand this well-known, productive relationship between Resilience and islands not as a one-sided process in which islands are reduced to 'blank spaces' or 'laboratories', and Resilience narratives are simply imported or imposed upon them, tried out and tested. The geographical form of the island and island cultures is doing important 'work' in these debates too. The key argument of this book is that island work and thinking do not simply follow in the slipstream of contemporary Anthropocene thought, but play a notable role in its development. Thus, we think it is important to understand the shift to reposition islands and islanders as exemplars of Resilience thinking as more than merely the by-product of a waning faith in modernity in the West. This would be to deny the work that widely held understandings of island life and island cultures themselves do in shaping Resilience ontologies and Anthropocene thinking. It would be to deny that thinking with certain geographical forms and cultures matters for the generation of thought and practice in the world. So, in the rest of this chapter, we will expand the analysis to engage with why and how Resilience thinking can be better understood once it is seen to be closely imbricated with an island ontology.

As noted in the last chapter, Darwin's understanding of the evolution of life profoundly overturned modern frameworks of reasoning associated with a telos of linear progress and a fixed human/nature divide, instead focusing upon how speciation and diversity emerges from the differentiating forces and co-relational entanglements which generate all life on Earth. As Cary Wolfe (2017), Timothy Morton (2017) and Stacy Alaimo (2010: 158), have all pointed out, Darwin's thinking with islands 'may have given us our first glimpse of the always already "posthuman"' (see also Alaimo, 2016). This is, of course, one key reason why many of these high-profile contemporary scholars are increasingly returning to Darwin in debates about the Anthropocene. But why did 
this radical new relational ontology emerge from Darwin engaging island life in particular? Because, for Darwin, islands - which are separate from the mainland and the homogenising forces of modernity - are seen to intensify or amplify relational entanglements and effects. Islands can therefore be understood as distinctive in that they are more clearly and obviously engines of differentiation or 'individuation' for the productive relational dynamics of life itself.

Thinking with islands was a central part of Darwin's development of the theory of evolution as a theory of diversification and differentiation, rather than a modern and hierarchical theory of linear progress (Quammen, 2018a). Darwin was extremely enthusiastic about how this 'island effect' was key to unlocking his revolutionary understanding of life, as his correspondence and personal notebooks regularly illustrate:

'Why is life short,' he asked, omitting the question mark in his haste. Why is reproduction so important? Why do animals of a given kind tend to be constant in form across an entire country but to differ at least slightly on separate islands? He remembered the giant tortoises on the Galápagos, where his stopover had lasted only thirty-five days but catalysed an upheaval in his thinking. He remembered the mockingbirds too ... Did creatures somehow become different when isolated? Put a pair of cats on an island, let them breed and inbreed there for generations, with a little pressure from enemies, and 'who will dare say what result', Darwin wrote. He dared. The descendants might come to look different from other cats, might they not? (Quammen, 2018a: 5-6)

It is well known that 'Islands have inspired a large number of scientists to develop key ecological and evolutionary theories.' (MacArthur and Wilson, 1967/2001; Borges, 2018: 1214; Patino et al, 2017; Mathews et al, 2019; Berry and Gillespie, 2019). It is commonplace to examine how species diversity is affected by the properties of islands and archipelagos (Triantis and Matthews, 2020). Important here is how evolution occurs in relatively isolated relational contexts; and how island separation from 
mainlands can be seen as intensifying or amplifying this power of relation and feedback effects; while modern frameworks of reasoning, in contrast, attenuate the importance of relation, flattening existence, homogenising life to modernity's disciplinary norms and values. As Elizabeth Grosz (2004: 7) says, in contrast to modern reasoning, Darwin's 'founding presupposition' is that as time and life move forward this 'generates more rather than less complexity, produces divergences rather than convergences, variations rather than resemblances'.

For Darwin, evolution, differentiation and the richness of speciation - revealed by thinking with the power of islands occurs through relations of co-dependency and this makes island life appear more creative and adaptive. As Elizabeth Hennessy (2019) has argued, the Galápagos islands where Darwin worked are emblematic sites of relational entanglement. Darwin's relational thought and understanding of life emerged from thinking with islands as radically alternative material sites of investigation. Since then, islands have become the most obvious differentiating mechanisms of life across the academic disciplines. Islands reveal how life is adaptative to its surrounding environment. In demonstrating this, the early or prototype posthuman relational ontologies developed by Darwin blurred the divide between life/ environment, pointing the way towards how all life on Earth can be characterised in terms of relational entanglements: as an interdependent, interactive, non-linear, processes of becoming.

\section{Interdependency}

In the seminal works of Darwin, island space is conceived as relational and in a constant state of becoming, and therefore as more dynamic and open to adaptive change. However, it matters how relational interaction is understood and how relations are put at risk or excluded. As we will see in this book, there are many ways of thinking about the key problematic of the Anthropocene and relational entanglements - on one level, not many people would disagree with the truism that entities are in relations with others, 
all life is dependent on other entities for sustenance, oxygen, warmth and so forth. Therefore, it is no surprise that within the massive range of Resilience literature today it is also the case that relational effects and implications can be grasped in different ways (Pelling and Uitto, 2001; Joseph, 2013; Chandler, 2014; Pugh, 2014; Evans and Reid, 2014; Grove, 2018; Wakefield, 2020). But what we wish to isolate analytically in this chapter is the productive grounding of engaging and drawing upon islands for today's relational thought in the Anthropocene, specifically as taken up in understandings of Resilience as adaptive change and transformation.

Central here is how relational interaction, highlighted in the key trope of 'feedback effects', is at the heart of island ontologies and epistemologies. What is it about islands that foregrounds the importance of feedback effects so powerfully? There are two related answers. First, is the high level of interdependency. Islands are (to varying extents) isolated from mainlands. This means that there is a greater dependency on immediate relations, and it is why islands, as noted above, are regularly characterised as 'paradigmatic places of human-environment relationships' (Kueffer and Kinney, 2017: 311; Bridges and McClatchey, 2009). Historically, in a crisis situation, you cannot just phone for deliveries or expect some external agency to intervene or assist. On the one hand, there is therefore the long-held trope of greater island self-reliance (Wilson, 1973; Goffman, 1978; Watts, 2018). This is the reason why, in modernist island tropes, such as that of Robinson Crusoe, the autonomous individual is foregrounded; and why those who have settled on islands more generally are said to demonstrate 'a long-term resilience borne of a basic human capacity to endure hardship' (Percival, 2008: 4). On the other hand, it is also clear that self-reliance is, in fact, a highly focused and situated dependency, or rather set of dependencies. Local resources and threats to these resources need to be understood in their specificity. Greater attention must be paid to the smaller and more tenuous modes of being of others. When time and resources are necessarily in short, contingent, or at least seasonal supply, life is necessarily one of adaptation. 
It is because of the need to focus upon these details and nuances of relations on islands that Laura Watts (2018: 198) defines being an islander as a 'shared experience of making practical ad hoc solutions to similar problems'. Here, for Watts (2018: 75-76), the islanders of Orkney exemplify the self-sufficient resiliency of islanders worldwide:

... infrastructure breakdown is just mundane, not cause for a social media meltdown. People shrug and clean out the grate in the stove and get it lit with a spare bag of coal. Many are on gas bottles or oil-fired cookers and heating, running from large buried oil tanks that do not blink when the electricity does. There are backup generators at the hospital, of course. Farmers and other businesses have invested in their own diesel generators and just keep going. I remember a nice story in the newspaper about a morning whip-round for some island generators to allow a wedding to go ahead that afternoon, despite an unexpected summer blackout. Surprisingly, on-grid wind turbines stop working when the power goes out (which seems like a major design flaw); wind turbines are not quite the road to self-sufficiency some might imagine. When the infrastructures considered essential to modern living fail, [Orkney Islanders] carry on with their modern lives, just wearing an extra jumper. Although communication, energy, and transport infrastructures are all but broken in the storm, civilization carries on. Despite what dystopian science fiction writers might suggest, when the lights go out, there is no apocalypse, no zombies, no drama. The Energy Islands are resilient, and suggest resilience is possible even when modern infrastructures are not.

Resilience ontologies draw heavily upon the idea that island life is by necessity relational, in the sense that survival is always a matter of being more than an entity, more than an individual, more than a set of fixed essences, tastes and preferences. For some commentators, this means that dynamic adaptive interdependencies literally force entities to 'become-other' - to hone and specialise their adaptive capacities in relation to other relations of 'becoming-other'. 
As Rubow (2018: 38) writes, with regard to how cyclones are experienced on islands:

There, on the ground, when sea and atmosphere evolve into a grand air and water pump, winds whip the waves white and force them into powerful cyclical movements that can reach 20 meters or more in the open ocean. Sea spray batters vegetation, rips foliage off trunks and branches and deposits them like a thick brown plaster on windward walls. In the low pressure on the ground, cars, roofs, stones, sand, windows, trees, doors and people enter an extreme, shaking state of culturalnatural hybridity in the Latourian sense in which humans and things are inextricably connected ... It may be possible to hold a 'modern' or 'global' perspective on things on a fine clear day, and at a distance to see a cyclone as a discrete weather-object. But when the loud howling noises, the invading waters and crushing boulders enter one's house, the hybrid mess of things and humans is impossible to overlook.

In this kind of work, island life is capable of enabling forms of thought and practice which do not rely on modernist abstractions of linear causality or illusions of empty grids of time and space. As Wolfe (2017) and Quammen (2018b) say, island forms of being and becoming literally take us into 'more-than-human' or processual and relational worlds, in ways that modern or 'mainland' experiences cannot so easily access. Such thinking is highly generative for contemporary Resilience design processes. For Robertson (2018: 50-51), the point is obvious when we think with islands: 'the ocean and its rhythms, the endless sound of the waves breaking on the reef, and the tides, constantly contracting and expanding around the islands like a heartbeat, feature in most aspects of daily life'. The observation becomes even more apparent for those who return to an island after living on a continent for a while:

When I enter the ocean, my indigenous identity emerges. I become a historical being riding waves, running as a liquid mass, pulled up from the deep and thrown forward with a deafening 
roar. I disappear with fish and strands of seaweed as I course through veins of the ocean currents ... Hitting that first wall of water, I become a Kanaka Maoli (Native Hawaiian) surfer. I ride waves; read the wind, swell directions, and tides; know the reefs and the seasonal sand migrations; and find myself most comfortable floating atop a board with my na'au (gut), mind, and heart facing the sea. (Ingersoll, 2016: 1)

Such examples are illustrative of how it is frequently said that Indigenous islanders in particular 'don't see nature as separate from people' (Lakpa Nuri Sherpa, quoted in Forest Peoples Programme, 2019). They offer 'a worldview that privileges not just the perspective of other men, but of other living beings-of trees, animals, oceans and stars.' (His Highness Tui Atua Tupua Tamasese Ta'isi Efi, 2018: x). Thus:

Climate has always been important for Māori. It affects the winds, waves, and ocean currents, influences which plants, trees, and birds are found in various parts of the country, and impacts the social, economic and cultural well being of individuals and communities. Through the generations Māori have built up extensive knowledge of local climate, from the character of local winds and rain to the forecasting of drier and warmer summers. These forms of knowledge have traditionally helped to make important decisions about the best time to farm, fish and navigate, among other activities. (National Institute of Water and Atmospheric Research, quoted in Percival, 2008: 13)

By contrast, '[i]n the developed world', Salick and Ross (2009: 138) argue that the 'loss of traditional cultures and perspectives has led to a disconnect between people and nature.' Therefore there is a close connection between imaginaries of tightly knit interdependencies of island ontologies and what is popularly understood as Indigenous cosmologies, which are similarly said to be immersed in practices of process and relation: 'Indigenous peoples have often been found to have intimate familiarity with the natural rhythms and processes of their ecosystem' (Salick and Ross, 2009: 138). 


\section{Feedback Effects}

Thus, we can see islands as not merely demonstrating the interdependency of all life, but, more importantly, particularly in terms of Resilience ontologies, the ways in which adaptation operates as an interactive process of mutual feedback. Here our main claim in this chapter is that drawing upon and thinking with islands and island cultures has become powerfully important for the development of Resilience approaches in the Anthropocene. The second and related aspect that it is important to highlight here is the meaning and importance of feedback for ontologies of Resilience. Feedback effects can be understood as intensifying relations between relations: binding life together in a process of interactive development. It is this process of interactivity - of mutual feedback effects - which enables some of the island capacities and affordances noted by Darwin, in terms of speciation, i.e. the differentiation or individuation of species.

Islands, as we have said, are significant sites for understanding relational entanglements as the overarching problematic of contemporary Anthropocene thinking. It is therefore not surprisingly that this focus upon 'the conceptual power of islands' has significantly intensified in recent years (Graham et al, 2017: 323). ${ }^{4}$ Islands as isolated communities of interdependency can intensify relations of feedback in relational entanglements, as small differences in climate, habitat or food ecologies can be magnified through a high level of interactive relation. Feedbacks are the way in which we understand the mediation of these multiple and ongoing interactions, as changes in environment or actions of other agencies evoke changes, in habits or behaviours, in other entities. Feedbacks then make the world or are a way of describing how the world makes itself or comes into being through relational interaction. In this way, islands can be understood as self-making communities. This is not autonomous selfmaking or autopoietic but, as indicated above, more accurately, a set of sympoietic communities of becoming and 'making-with' (Haraway, 2016: 58). 
In developing this point it is useful to highlight that the endorsing of a relational Resilience ontology does not necessarily imply being anti-technology or against the human repositioning or redirecting of relations. Despite what could be inferred from the strong focus upon resilient Indigenous islanders in these debates, it is important to grasp the core analytics of contemporary Resilience in the Anthropocene. Neither does Resilience thinking with islands today align well with that of The Island of Dr. Moreau and H. G. Wells' 1896 version of Darwinian naturalism (2005), which comes with the moral lesson that humans should not interfere with 'nature'. For, as we have said, in contemporary Resilience discourses the key analytical point is that there is no strict human/ nature hierarchy, and therefore no pure and separate 'nature' to be interfered with. The more-than-human is always already relationally entangled with the human after the end of the world once the environment could not be stood apart from and grasped by way of modern framings of a human/nature separation. Thus, many contemporary Resilience approaches reposition and adjust feedback effects and rework relational entanglements in new ways. It is here that island life takes on even more important purchase and power, becoming generative of new ways of being resilient in the Anthropocene.

For example, in 2017, during the Tallinn Architecture Bienniale (TAB), ecoLogicStudio curated and designed an exhibition entitled Anthropocene Island (ecoLogicStudio, 2017a). This project involved architect-researchers, artists and scientists looking at the former Soviet military base at Paljassaare, on the contaminated peninsula in Tallinn. This is the site of a large wastewater treatment plant and landfills, and has been designated part of the European Natura 2000 network as an important nesting site for migratory birds. Concerned that understandings of the Paljassaare Peninsula were being shaped by two outdated and conflicting ideologies - on the one hand, the site as an illusionary wilderness; and, on the other, commercial development into an ideal green city - Anthropocene Island sought to challenge these ways of understanding human-environment relations as 'deeply 
conservative' (ecoLogicStudio, 2017a). Framing the site as existing at the 'inevitable frontier of future urbanity' (ecoLogicStudio, 2017b), Anthropocene Island 'speculates on how from such a controversial site the origin of a new notion of bio.City may emerge' (ecoLogicStudio, 2017a).

The central thrust of the project was that island life exemplifies the creative or 'emergent' powers of life that cannot be accessed directly by way of modern frameworks of reasoning. The curators argued that when we view islands from the different perspectives of orbiting satellites, or micro-organisms, we see that islands are composed of intricate webs and assemblages of human and non-human co-relations. Anthropocene Island instead sought to develop:

... a non-anthropocentric view of the urban. From this perspective cities and their morphologies are mostly determined by flows of matter, information and energy that fuel their metabolisms. This shifts our attention from looking at urban form (figure ground) to the morphogenetic process that underpins the current morphology of an urban landscape: we can look at cities as living systems. (ecoLogicStudio, 2017c)

Anthropocene Island was about working with, enhancing and designing systems of biosensors, membranes and 'digestive apparatuses' which enhance Resilience within the complex human and non-human relational entanglements of the Anthropocene. Bringing together disciplines of biology, computation and urban design, and organised through a range of scales from the vastness of the Baltic Sea to the micro level of algae, Anthropocene Island explored the possibilities for designing new 'resilient topographies' (Barnett, 2017) which:

promote a new urban morphogenesis whereby Tallinn's urban wastewater infrastructure deeply affects the biotic substratum of the peninsula. The resulting 'contamination' becomes a morphogenetic force, inducing an artificial hyper-articulation of the landscape and its living systems which will evolve into a digestive 
apparatus or membrane. Pathogens are re-metabolized, diluted or captured by augmented ecosystems; infrastructural networks thicken into filtering surfaces, which in turn fold into convoluted epidermis populated by a large amount of biochemical reactors. (ecoLogicStudio, 2017a)

The purpose here is to understand islands as living, interdependent, interactive systems of human and non-human co-relations and feedback effects. In thinking with islands as important sites of relational entanglements, a different and more dynamic ontology becomes apparent which feeds into the development of contemporary analytics of Resilience.

\section{Immanent Life}

In Resilience ontologies, islands are often understood as isolated systems where relations of interdependence and interactive feedback establish an internal set of immanent processes which shape or guide the direction of emergent causality. It is the differences between islands and their unique systems of inter-relation that come to the fore, as they did for Darwin and many others, rather than universal laws of development or coexistence. Life itself is seen to work in island ways; where differences make differences and life appears as the interactive power of differencemaking, differentiation and individuation. Thus, while there may be universal laws of nature, these can be grasped only in abstraction; in concrete contexts it is the interactive and lively effect of individuation that is the most important aspect. Thus, relational approaches often draw upon imaginaries of island systems of close interdependencies, enabling them to emphasise the importance of context, of relationships, of the powers of entities to affect and become affected, rather than thinking of entities in terms of essential properties and fixed causal paths in empty grids of time and space.

We wish to emphasise that this ontology of interactive life foregrounds immanence: stressing relationships as having a 
generative and creative power rather than merely being expressions of Newtonian mechanical causality. In immanent framings of life, entities make a difference not as autonomous self-making rational subjects, but as collective interacting agencies joined by virtue of the fact that their interaction is itself the process of 'life'. In thinking with islands, as we have shown, entities are always 'more-than' isolated entities with inherent properties, but always in relation and always 'becoming. This is because they are constantly adapting to, affecting and being affected by, other agencies and entities. Concomitant with this interaction, the environment then not only shapes the becoming of entities, it is the becoming of entities through their interaction. The environment, in an immanent ontology, is no longer a passive object or background but active and indistinct from the actors in the foreground. In experiencing life as interactive, the core binary divides of modernist or mainland understandings become blurred and indistinct, and therefore increasingly problematic. These are the divides between figure and ground, subject and object, agent and structure and organism/entity and environment. Island thinking - which foregrounds thinking with interactive relations - is not merely a matter of adding more things or entities to concerns but crucially provides a different ontology of the world.

The contemporary framing of debates and forms of Resilience, such as the example of Anthropocene Island, centre upon the immanent interactive potentialities of life itself - an approach that is not merely exemplified by island life, but which, as we have seen, is analytically derived from thinking with islands. This represents an important change in direction from the earlier sustainability approaches we noted above, which sought to contain the radical shifts heralded by the Anthropocene and to maintain existing forms of life to ensure a 'happy ending' for modernist ideals of progress (Tsing, 2015). Today, the focus is increasingly moving in the direction of 'staying with the trouble' (Haraway, 2016), stressing contingency and the work of governance as one of continual care and responsivity. Indeed, in the relational ontologies of Resilience, there is increasingly less of a focus upon islands and islanders 
existing as harmonious, self-regulating adaptive systems, and more upon the work that this requires of islands and islanders: harmony is the product of constant attention to new configurations, threats and opportunities. Thus Resilience is not so much about ways of using resources most efficiently or sustainably but of becoming sensitive to changes and shifts in environmental relations: a way of coping or living on after 'the end of the world'.

One particularly good example of this is Watts' (2018) acclaimed book Energy at the End of the World: An Orkney Islands Saga. For Watts, the key thing about island life is that the environment is not passive, but one of processual, immanent becoming, which blurs the binary between humans and nature. However, Watts is less focused upon 'happy endings' and more concerned with life in the Anthropocene as a condition which we are all already in; one requiring a pragmatic alertness to the need for constant adaptation. If there is hope here, then this remains in the creative potentialities of everyday life, which, as we have already noted above, for Watts, are exemplified by island life. But here the approach has shifted to one of pragmatic world-making, rather than of tapping into the power of self-regulating adaptive systems.

Watts' central illustration of this is what she calls the 'Orkney electron', associated with the generation of renewable energy and power through the Orkney archipelago. As Watts (2018: 65) says, '[e]lectrons are always tricky to think with. They exist in lightning strikes, interconnector cables, amber resin, envelope glue, light bulbs, hydrogen atoms, electrons as spinning particles, and electrons as waves of probability'. Her key point is that when we focus upon electrons in this constant state of becoming, '[p] ower is no longer a story just about scale, centralization, or development' (Watts, 2018: 45), but also, more fundamentally, about tracking the emergent effects, disturbances and frictions (material, political and otherwise) which generate Orkney electrons. As Watts (2018: 75) writes, these 'are tangible in the Energy Islands, but not just because I can touch the national grid cable ... You can feel Orkney electrons in the sheer cold wind ...' they emerge in the 'undersea power lines between the islands, and in the cables 
strung up over the heather.' Watts' particular framing of island life in these explicitly more-than-human terms of becoming, like the other approaches to Resilience ontology discussed above, clearly poses a direct challenge to modernist separations of human/nature and subject/object. But, in her work, Watts seeks to go further in following the disturbances and emergent effects, understanding (island) life more as an open and contingent process of becoming than as contained or bounded self-regulating system.

To be clear, Watts is certainly focused upon the resilience of islands and islanders and is particularly concerned with how they can better harness renewable energies. But she also extends or reworks Resilience as an ontology in a different way from the previous examples discussed in this chapter. For Watts, as noted, the Anthropocene is a condition we are within rather than one we observe from the outside, and this shift in perspective leads to jettisoning the notion that (island) inter-relations could be viewed as if they were bounded and discrete self-regulating systems, or that Resilience approaches could be rolled out in an instrumental way of holding back planetary change. For Watts (2018: 127), it is too late to restore the human as subject separate from and directing the world as object; the change has already come, and we therefore now need to learn new ways of creatively 'stay[ing] with the trouble.' One notable illustration of this is Watts' employment of what she calls a fictional cyborg, in the form of the 'Electric Nemesis', who becomes her guide throughout her fieldwork to a deeper, more fundamental, understanding of (island) life itself in the Anthropocene.

Holding a ball of twine in hand, Watts (2018: 369) is taught about how 'the creative possibility is in the refiguration of existing things and materials (string, gut, bacteria, patterns, knots), artful integration work.' The Electric Nemesis is a guide to understanding that everyday (island) life is generative, creative and worldmaking. But this is not necessarily a harmonious, self-regulating system, which leads to a better ordering. The focus is upon the disturbances and emergent effects of co-relational entanglements. On the islands of Orkney, as Watts points out, these are expansive 
and work well beyond the island boundary - through the politics of Westminster, the role of venture capitalists living in North America, or the European Union, which funds renewable energy projects, as well as tidal forces, wind patterns and particular island geographies. For Watts, there is therefore a need to regularly intervene, often intensively, to carefully cultivate and amplify the already creative capacities of island life that exist within often vast relational entanglements.

Here, Watts (2018: 350) takes a pragmatic approach, arguing for 'a self-determined, decentralized solution that is appropriate to the place: reconfiguring and reweaving the local energy network with what is at hand. In this case, tying it together with electric cars, council-run charging points, the need to resolve fuel poverty, and an imaginary that is not constrained by an overheating smart grid.' But, for Watts, thinking with islands is not about producing detailed models and replicable programmes of Resilience which can be transferred off island to elsewhere. On the contrary, thinking with islands is more of an ethos or ethic of world-making; for acting in the world differently. As the Electric Nemesis says to Watts (2018: 370) at the end of her book:

'The saga is ending. But you cannot take the islands with you. You can only take me.' She leans forward, and I swallow hard, certain I can smell every rotting stich and suture that holds her bruck flesh and folklore guts together. 'I am made of the Energy Islands, remember. I am the saga. Let's go see what we can do with something other than a bit of Orkney salted string ...

Such contemporary approaches do not design intricate models of Resilience by analysing island cultures and islander ecosystems, which could then be replicated, 'off-plan', in order to give continental, 'mainland', Moderns a chance to rein in climate change and environmental instability. For Watts, this would be to go against how things already are in the Anthropocene. She instead employs islands as a 'living laboratory' (Watts, 2018: 105) for generating a new ethos or praxis which focuses upon the active and dynamic powers of life itself (exemplified, above all, by island 
life); understood in terms of its immanent, generative and creative potentialities. In a development of earlier and simpler ontologies of Resilience, here the spatial-temporal imaginary is expanded and thought through more openly. Resilience is not seen to operate in flat space-time, so that order and self-regulating harmony increasingly emerge out of chaos. Rather, Watts forwards a pragmatic approach to island- and world-making, where it is the ascribed immanent potentialities and pragmatic creativity of island and islander life which generates hope for such thinking in the Anthropocene:

[T] he saga I am telling is not another end-of-the-world climate fiction. It is not a prepper's saga of how to survive some coming apocalypse. It does not grind to a halt in the face of capitalism's rapacious devouring of the planet's resources. The Orkney electron gives me hope that the future can be otherwise, that there is another way of being and living that is not apocalyptic. The Orkney electron tells me the end is not nigh. There are some people who are just getting on with making a low-carbon and renewable energy future, centralization be damned, the rules of capitalism be damned - even while they are within and reliant on both. (Watts, 2018: 123)

Instead of investing hope in top-down, modern frameworks of reasoning, or linear narratives which coherently play out over time, these approaches allow for more flexible responses, rather than intervening directly to tackle root causes or engineer outcomes on a larger scale. They 'facilitate', 'enable' and work hard to 'cultivate' existing powers and capacities, seeking to redirect them to new possibilities for 'staying with the trouble' after the crisis of faith in modern frameworks of reasoning. They are intensive, but not in top-down, command-and-control ways. The Electric Nemesis is insistent on this point in her guidance throughout Watts' (2018: 126) saga, cautioning against the 'hubris' of modern ways and those who speak in terms of transferable models; saying to those who aspire to a god's eye view of the world 'I smell them! I always smell them! God-trickers!' (Watts, 2018: 365) 
This move to develop or expand the relational ontology of Resilience from a tightly contained self-making system - where (island) life is articulated as a complex problem-solver which takes us towards ever more efficient systems - illustrates a move along the heuristic continuum, established in the Introduction, towards the Patchwork ontologies which are the focus of Chapter 3. For whilst, as we have explored, Resilience breaks with modern frameworks of reductive linear reasoning, unlike many of the other approaches to Resilience discussed in this chapter, Watts does not tend to reify the world and suborn us to it. In her approach, we make, journey and explore the world through creative and often contingent forms of refiguration, rather than merely reflecting upon and becoming aware of relations so as to 'bounce back' better. It is true that Resilience is her key concern, but Watts also radically opens up the innovative possibilities associated with the relational affects and knots of co-relational entanglements; pushing further away from notions of an immanent telos, offering an alternative mode of goal-directed transformative change. As we will discuss in the next chapter, Patchwork ontologies characterise the work of many contemporary scholars, experimental artists, designers and activists concerned with the Anthropocene.

\section{Conclusion}

In this chapter we have analysed how Resilience relational ontologies draw upon, engage and think with islands. These challenge top-down approaches of command and control, which seek to save islands and islanders by way of modern frameworks of reasoning; instead, focusing upon the dynamic potentialities of adaptive interactive life itself (exemplified by island life). The first section focused upon how islands and island cultures are key figures for Resilience thinking, while the following sections went further and drew out how Resilience thinking can be analytically understood as being derived from thinking with islands. Here, Darwin's theory of evolution - enabled through his island experiences was shown to have been historically key to an understanding of 
life as an interactive and adaptive process of becoming. Darwin profoundly disrupted modern frameworks of reasoning, the hierarchical understanding of the human/nature divide, and a telos of linear progress and hierarchical development. For Resilience thinking, this understanding of life is a highly productive resource to be drawn upon. It enables us to think of life itself as emerging through interactive, processual becoming; through an immanent trajectory of ordering and ongoing adaptation. In this way, Resilience draws upon island life as a system of complex and dynamic organisation. This, as we examined, has done much in international debates to invert the relationship between mainlands and islands; so that today, the argument often goes, we can all learn how to be more resilient by paying greater attention to islands and islanders. Whilst this has been notably generative for promulgating Resilience as a relational ontology, towards the end of the chapter we also began to map out how, for some commentators, the ontological stakes have extended beyond tightly constrained or closed-system imaginaries towards the more open ontology of Patchworks, which we turn to in the next chapter.

\section{Notes}

${ }^{1}$ As O’Brien (2017: 43) says: 'A common way to imagine environmental futurity in the early decades of the twenty-first century is through stories about resilience. At a time when the concept of sustainability has largely given way to a sense of recurrent crisis, narratives of successful adaptation have powerful currency'.

2 Thus, for Wu et al (2019: 1), 'the island is an example of a coupled human-environment system ... which is integrated at the local (intracoupled), regional (pericoupled), and global (telecoupled) scale. This has important implications for the position of islands in debates about the Anthropocene. For example, for Vitousek and Chadwick (2013), '[a]lthough the islands of remote Oceania were among the last places reached by humanity, many islands entered the Anthropocene early. Extinctions - some caused by the first people to discover islands - have been far more frequent on islands than continents, and the intensity and consequences of human-caused 
biological invasion, deforestation, and landscape alteration have been substantially greater as well ... [Therefore], islands provide a useful model for understanding how coupled human and natural systems experience the Anthropocene.' As another example, Holdaway et al (2019: 17) focus upon Ahuahu (Great Mercury Island), New Zealand; saying that 'The lateness and prominence of Polynesian colonisation of New Zealand make it an ideal place to investigate the Anthropocene [and, in particular, to study] ongoing human-environmental interaction. Elsewhere in the world, a lengthy history complicates the ability to differentiate between the impact of people on the environment and the consequences of engagement. [Island] characteristics provide the scope to study the impact of engagement where it is particularly discernible.'

3 To further illustrate, Barnett (2001: 979) focuses upon island resilience research in the Pacific, examining the need to engage the 'whole island systems where the full gamut of biophysical, social, and biophysicalsocial interactions are taken into account ... to shift from the study of the parts to a study of the whole ...'

${ }^{4}$ As Graham et al (2017: 323) write, 'Islands are widely considered to be model systems for studying fundamental questions in ecology and evolutionary biology. [Here, debates about the Anthropocene] exemplify the historical and continuing importance of islands .... Thus, in recent years, there has been a proliferation in the field of island, Anthropocene and evolutionary biology research (for examples, Helmus et al, 2014; Leppard, 2018; Salinas-de-León, 2020). 



\section{Patchworks: The Ontology of the World}

\section{Introduction}

In ontologies of Resilience, relational interaction is seen to be underlying or immanent to the constitution of 'life' itself; something which is revealed particularly well through the experience of bounded island modes of interaction and adaptation. Here, (island) life is articulated as a complex problem-solver, a little like the market in neoliberal discourses; held to possess immanent organisational powers of bringing order out of chaos. For discourses of Resilience, a key trope is that this power is always self-organising, rather than being controlled or guided by some external or transcendental agency. Complex life is understood as becoming more efficient and adaptive rather than increasingly disordered and entropic. Focusing upon the whole island ecosystem as a bounded laboratory for revealing potentialities of interactive life, islands have become key symbols of 'hope' for many commentators in debates about the Anthropocene (Mission Blue, 2019). The widespread argument, particularly in more managerial-oriented debates, is that the rest of the world can and should learn from the 'indelible resilience' (Nicks, 2017) of islanders.

How to cite this book chapter:

Pugh, J. and Chandler, D. 2021. Anthropocene Islands: Entangled Worlds. Pp. 69-108. London: Ubiquity Press. DOI: https://doi.org/10.16997 /book52.c. License: CC-BY 4.0 
However, as we explored in the previous chapter, a growing number of commentators seek to extend and problematise this relational ontology. For contemporary approaches, such as those of Laura Watts (2018), there is something a little too homogenising about a Resilience ontology of relational interactive life; with its mutual and ongoing adaptations and its 'happy endings' of ever newer and more efficient emergent orders. One way of grasping this is to see Resilience as only a limited break from a modernist causal ontology, where even though interdependency and interaction are stressed it appears that there is a 'hidden hand' guiding the direction of a new telos. Often this alternative telos of 'progress' and 'development' is read to start with the explosion of life from the Big Bang (Kurki, 2020) right up to our complex and differentiated present. However, this perhaps too easily assumes that we are all in the same boat, guided and shaped by the same underlying forces and sharing the same ecosystem and the same planet. Many advocates of Resilience ontologies engage islands by drawing out similar tropes of relational harmony and self-organisation, from which, it is widely claimed, the rest of the world can derive important insights. This holistic and beneficent framing often aligns Resilience ontologies with romanticised notions such as those of Gaia (Lovelock, 2007) or 'Earth island' (Earth Island, 2019).

The Patchwork ontologies examined in this chapter draw upon and develop island thinking as relational ontology beyond the perceived limits of the holistic and interactive systems approach of Resilience. In Patchworks, the modernist imaginary of islands existing in a flat, two-dimensional space, side-by-side, tracing continuities in relation across linear time into the ever more efficient self-regulation of Resilience, is replaced with a more open and disruptive island ontology of spatial and temporal becoming; destabilising the 'solutionist' or instrumentalising aspects of Resilience, making Patchwork approaches less governmentalising and human-centred. Patchwork ontologies focus less on adapting to pre-existing processes or powers, than on practices of bringing into being, of engendering or inculcating relational ways of becoming in the world. By drawing upon islands in this way, 
Anthropocene thinking becomes a more open, less controlling, process of pragmatically 'giving-on-and-with' (Glissant, 1997: 142) the unpredictability of emergent disturbances and effects. Patchwork ontologies spatially and temporally open out an island ontology, understanding life in terms of patchwork islands of relational assemblages, knots and nodes of disturbances and effects. Thus, by drawing upon and working with islands, Patchworks becomes a new relational ontology of 'world-making', moving beyond the human/nature divide.

The chapter is organised into four sections which elaborate the analytics of Patchwork ontologies and how they draw upon islands and island cultures. In the first section, we examine how anthropologists have long had an interest in how island cultures from around the world relate to their environment in different ways from Moderns (Mead, 1957, 2001; Strathern, 2004, 2020). It is the focus upon the contingent and unpredictable powers of relational disturbances and effects associated with island life which marks Patchwork ontologies and, as noted, this means that Patchwork approaches cannot be easily exported as a set of instrumentalising techniques or practices, as in the managerial ontological imaginary of Resilience. For example, in the Patchwork ontology of Anna Tsing (2015), developed by thinking with Japanese islander practices of satoyama, there is less of a focus upon predictability and intentionality, and more on how relational entanglements and feedback effects can be surprising and unintentional, and therefore creative and productive. Thus, as the second section elaborates, the world is seen as lively and full of unexpected possibilities. From thinking with islands in contemporary design practices (Daou and Pérez-Ramos, 2016), to Phil Hayward's (2012a, 2012b) conceptualisation of the 'aquapelago', developed by work on Haida Gwaii (off the northern Pacific coast of Canada), we examine how Patchwork ontologies characterise a broad range of burgeoning experimental contemporary approaches to anthropology, ethnography, the arts and design, all of which draw heavily upon islands as a key resource for contemporary Anthropocene thinking. 
In the third section we explore how Patchwork ontologies expand thinking with islands in the Anthropocene so that island ontology is increasingly imported into an understanding of the quotidian and everyday. Employing the works of Glissant (1997), Brian Russell Roberts and Michelle Stephens (2017), Mimi Sheller (2020), Godfrey Baldacchino and Eric Clark (2010), Teresia Teaiwa (2007) and Deborah Bird Rose (2017a, 2017b) as examples, we discuss how islands are configured not as worlds that we are merely in or on, there to be managed and adapted to; they are also ways of expressing and understanding our own processes of world-making. In this framing, islands are not so much the outcome of a process or relational ontology, as the process of becoming or of movement itself (Glissant, 1997). We examine how, in Patchwork ontologies, working with islands becomes a practice of opening ourselves to the world. This experimental set of infinite openings contrasts with the use of relational ontology at the other end of the ontological continuum, of Resilience, which tends to reify the world and suborn us to it within bounded self-regulating systems. The final section of the chapter turns to how, for many contemporary Anthropocene thinkers, this process of world-making, frequently emerging from engaging and working with islands, is generative for an ethos or duty of care (Spahr, 2005; Bird Rose, 2017a; Wetlands Wanderers, 2018).

\section{A Patchwork World of Islands: Disturbances, Emergences and Relational Affects}

Before turning to what we mean by Patchwork ontologies explicitly, it is useful to examine why islands and island cultures are such an important resource and reserve for non-modern thinking more generally today. At least since Margaret Mead (1957, 2001) played her pivotal role in shaping the discipline, in the 1950s, anthropologists have been fascinated by how islanders understand their relationship to the world differently from modernist or 'mainland' thinking. Here, for Marilyn Strathern (2004), we do not need Donna Haraway's relatively recent addition 
of the 'cyborg' into Western critical theory to help us realise that many people across the world, exemplified by the Melanesian islanders, the interlocutors for Strathern, do not construct their existence in terms of modernity's human/nature divide. What Strathern (2004: 118) calls these 'Melanesian cyborgs' see themselves as inextricably part of relations, where '[o]ne person or relationship exists cut out of or as an extension of another. Conversely, these extensions - relationships and connections - are integrally part of the person. They are the person's circuit'. This is widely reflected in Melanesian island culture so that:

There is no difference between shell strands and a matrilineage, between a man and a bamboo pole, between a yam and spirit. The one 'is' the other, insofar as they equally evoke the perception of relations. The different components or figures are thus all parts of persons or relationships fixed on to one another ... [For example] the flutes that both are children and produce children, or spirits that are both within and beyond the body-form of persons. Melanesians have a cultural facility for presenting their extensions of themselves to themselves, a facility for, we could put it, moving without travelling (Strathern, 2004: 118).

For Strathern (2004: 118), these islanders are therefore nonmodern through and through - '[t]he distinction between the Melanesian cyborg and Haraway's half human, half mechanical contraption is that the components of the Melanesian cyborg are conceptually "cut" from the same material'. What is key then about Melanesian island cultures (and, as we will shortly see, for the development of more recent Patchwork ontologies in Anthropocene thinking) is 'the creative act of severance, the burst of information that makes one person visible as an extended part of another' (Strathern, 2004: 118). Thus, for Strathern (2004: 118), it is not merely that people and things are cobbled together as hybrids or cyborgs of human-non-human relations; rather, what exists on the island already emerges from the 'perception of the common background to all movement and activity'. 
Similarly, for Deborah Bird Rose (2017a), there is a shared prefiguring relationship in the island cultures of Australia which she studied. Bird Rose (2017a: G52) draws out how the more-thanhuman is the starting point for these cultures, the beginning for understanding (island) life, and not something which is to be only factored in after some critical reflection:

Of the many stories one might tell about multispecies connectivities, the starting point for me is in Aboriginal Australia, where I have been learning about multispecies kinship and connectivity for many years. The stories might be said to begin 'in the beginning' with the Dreamings, also known as the creation ancestors. The Dreamings are the creators of much of the biotic life of earth. They are the shape-shifters and are the founders of kin groups. Those kin groups include the human and the nonhuman descendants of the ancestors.

For Jun'ichiro Suwa (2007: 6), commentating upon island cultures from Japan, there is also already a prefigured more-than-human world from which entities and peoples derive their connections to each other:

The Amami Islands of southwestern Japan are marked by their population's deep attachment to their own shima, as enacted through various practices and performances of demarcation. Each shima is a work of territorial imagination, an extension of personhood and a 'cultural landscape'. In this sense, a shima is a sanctuary, in that the natural environment and social space are articulated by the performative in such a way that one imagines them as a totality. Islands are both the ground and product of cultural practices and threats to their viability can thereby be construed as threats to human security more generally.

In these anthropological studies, island cultures offer us insights into worlds which cannot be reduced to the binaries that sustained the modernist imaginary (subject/object, mind/body, human/nature divides). ${ }^{1}$ Importantly, their starting point is the flux of relational interaction, in which fixed entities are much less 
distinct and stable; inspiring understandings of the everyday and the ordinary as intricately co-related. Thus, for many contemporary commentors, they help us face the Anthropocene as the 'Age of Entanglement' (Daou and Pérez-Ramos, 2016: 9).

Bruno Latour (2017) captures the stakes of this shift well in his particular reading of Gaia. As Latour (2017: 81) says, many people are not aware that the theory of Gaia, in both Hesiod and James Lovelock, was developed by thinking with islands:

We have all read Lord of the Flies, the story of some young British schoolboys marooned on a desert island from which they can no more escape than we can from our blue planet, and on which they slide little by little down the slippery slope that leads to barbarity. It so happens that its author, William Golding, was Lovelock's neighbour in a little Wiltshire village with the delightful name Bowerchalke, and it is to Golding that Lovelock owes his theory. ${ }^{2}$

This matters a great deal. Against readings of islands as bounded spaces of self-regulating harmony, for Latour, Gaia is a more troubling figure, leaving us with a very different moral lesson for the Anthropocene, particularly when we go back to the Greeks and Hesiod: ' $[w]$ hat is certain is that she is not a figure of harmony' (Latour, 2017: 82). Here, working with islands, with Gaia, invokes a different imaginary for Latour, concerned with how the planet is not there 'for us', to enable human flourishing as a goal; instead the 'intervention of Gaia' is associated with humbling and increasingly unpredictable forces (see also Stengers, 2015). This approach not only foregrounds multispecies connectivities but also the disturbances and emergent effects of human-nonhuman relations which can no longer be understood, managed and directed in the governmental understandings of Resilience (Latour, 2017).

For many such Anthropocene thinkers today, working with islands in this less controllable and predictable way - but, importantly therefore, more creatively and experimentally - shines a powerful light upon the hubris of modern ways of thinking (Tsing, 2015; Watts, 2018). It offers us alternative, more generative, ways of thinking through the central problematic of relational 
entanglements, and for engaging the Anthropocene as a condition which we are all already in. As Tsing (2015) makes clear in her highly influential The Mushroom at the End of the World, working with islands and islanders brings to the fore the localised figurations and co-shaping of relations which cannot be grasped by formalised and abstract modern reasoning and interventions. Tsing's (2015) study of Japanese islanders cultivating the matsutake mushroom is a contemporary example of Patchwork ontologies coming to the forefront of contemporary thought.

Exploring the relationship between people, landscapes and mushrooms, Tsing follows the commodity chain of the matsutake mushroom from North America and China to the islands of Japan. In contrast to what is seen as the modern hubris of North American and Chinese practices which separate humans from nature, for Tsing (2015: 151-152), it is above all the Japanese concept of satoyama woodlands which offers us the most hope in the Anthropocene:

Satoyama are traditional peasant landscapes, combining rice agriculture and water management with woodlands. The woodlands - the heart of the satoyama concept - were once disturbed, and thus maintained, through their use for firewood and charcoalmaking as well as nontimber forest products. Today, the most valuable product of satoyama woodland is matsutake. To restore woodlands for matsutake encourages a suite of other living things: pines and oaks, understory herbs, insects, birds. Restoration requires disturbance - but disturbance to enhance diversity and the healthy functioning of ecosystems. Some kinds of ecosystems, advocates argue, flourish with human activities.

For Tsing, humans and other forms of life are intricately entangled through such islands of interconnection, which are brought to the surface via momentary or contingent disturbances and effects, and each island requires the care of constant and delicate reconfiguration to engender these creative processes. Work within island Patchwork ontologies shifts the focus to concrete interactions in specific moments, often via rich ethnographic research, 
that enable us to see the creativity in the everyday (see also the discussion of Watts 2018 in the previous chapter). Thus, an islander approach, for a Patchwork ontology, is understanding oneself to be part of, but not directing of, processes of creative emergence at local and micro levels. As Tsing (2015: 152, emphasis in original) continues:

Ecological restoration programs around the world use human action to rearrange natural landscapes. What distinguishes satoyama revitalisation, for me, is the idea that human activities should be part of the forest in the same way as nonhuman activities. Humans, pines, matsutake, and other species should all make the landscape together, in this project. One Japanese scientist explained matsutake as the result of 'unintentional cultivation, because human disturbance makes the presence of matsutake more likely - despite the fact that humans are entirely incapable of cultivating the mushroom. Indeed, one could say that pines, matsutake, and humans all cultivate each other unintentionally. They make each other's world-making projects possible. This idiom has allowed me to consider how landscapes more generally are products of unintentional design, that is, the overlapping world-making activities of many agents, human and not human. The design is clear in the landscape's ecosystem. But none of the agents have planned this effect. Humans join others in making landscapes of unintentional design.

This focus upon 'unintentionality', 'effects' and 'disturbances', rather than instrumentality, is clearly different from the 'solutions-thinking' of those who seek to draw upon and develop 'island powers' of Resilience. Indeed, for such Patchwork island approaches as Tsing's, solutions-thinking would be a barrier to the need to be constantly attuned, alert and responsive to emergent effects. Neither is the power of interactive island life understood in terms of self-regulating, harmonious systems which tend towards order. The promise of 'order' or 'solutions' would be too modernist, denying our entangled responsibilities and commitments, while greater sensitivity to effects and disturbances enables us to become increasingly aware of them. 
The key word for Tsing (2015: 160) is 'disturbance' of human and non-human relational entanglements, which is not understood as negative but rather positively framed as opening up the possibilities for new co-relations to emerge. Bearing close relation to Strathern's (2004) research on islands and island cultures noted above, disturbance of relations is 'a beginning, that is, the opening for action. Disturbance realigns possibilities for transformative encounter. Landscape patches emerge from disturbance' (Tsing, 2015: 152). Responsivity to disturbances and the emergence of landscape patches are positively contrasted with modern processes of command and control, where:

... humans were not part of forest assemblages in matsutake management in the United States and China; managers there leaped to anxieties about too much human disturbance, not too little. In contrast, too, to satoyama work, forestry elsewhere was measured on a yardstick of rational advancement: could the forest make futures of scientific and industrial productivity? In distinction, a Japanese satoyama aims for a liveable here and now. (Tsing, 2015: $162-163$, emphasis in original)

Such approaches are heuristically or analytically similar to the traditional Japanese gardens which first appeared on the island of Honshu around $600 \mathrm{AD}$, where Buddhists developed a new style of gardening by working with care, and 'intensively', to attune to disturbances and emergent relations on the island, rather than working to a preset plan. Patchwork ontologies focus upon patches or islands of creativity and refiguration forming in nodes or knots of assemblages across time and space. The ontological assumption that all forms of being emerge through webs or networks of co-relation puts the emphasis on creative crossings and interconnections, meaning that new opportunities arise to see with and through these relations and co-dependencies. These are relations of 'affordance' and 'affect'; when some entities or processes are affected by others, they can be seen as 'networked' or 'assembled', but they have no relation of immanent or linear causation which can be mapped, reproduced or intervened in, as in 
Resilience (see Latour, 2004). Tsing's is an example of a Patchwork approach which draws upon and works with islands after the end of the world (as a coherent object which could be managed or controlled by way of the nature/culture schema).

\section{A Lively World of Islands}

At this point it is perhaps worth briefly restating that our argument is not that Patchwork ontologies, or Resilience ontologies, should be reduced to 'island thinking.' There is no such thing as 'island thinking', only variations of ways of drawing upon and working with islands in different places and at different times in history. As we noted in the introductory chapter, in European and modern thought, the island was more often understood very differently; as insular, isolated, and backward when compared to continental, mainland reasoning (Gillis, 2004). What we are highlighting in this book is that islands are important sites for Anthropocene thinking, ${ }^{3}$ partly because of their marginalised or liminal position within modernity and the fact that non-modern attributes were often projected upon them. After the supposed closure of the modernist imaginary of progress, these attributes have come to the forefront in the search for alternative forms of thought and practice in the Anthropocene. This shift has enabled island associations with Patchwork ontologies to be a major influence on many contemporary design processes associated with the Anthropocene. Daou and Pérez-Ramos (2016: 8) describe how 'the island [has become] a design tool, in scales ranging from gardens to cities to regions'. This is because, for these authors, more than any other geographical form, working with islands:

allows us to better understand the interactions between things and the world and also to construct new forms of thought that help reveal the world and render it legible. Precisely by transcending the dichotomy between interior and exterior the island avoids slipping into particularism, and becomes instead the figure through which a new form of universalism can be conceived. 
In this way, the island bolsters the ecological imaginary, helping design face an entangled world. (Daou and Pérez-Ramos, 2016: 9)

For the Patchwork approaches of world-renowned garden designers, like Gilles Clément, 'the island as both a physical manifestation and symbolic representation has significantly influenced' their work (Herrington and Lokman, 2016: 144). Examples of this include Clément's design of Parc Henri Matisse in Lille, and the concept of 'Garden in Motion', which forces 'designers to break down long-standing conceptions of gardens and landscapes as simply governed by human processes and needs' (Herrington and Lokman, 2016: 145). Such approaches conceptually and practically work with the relational entanglements and feedback effects of islands to design gardens as 'open-ended processes', reconfigured, for example, 'as a seed bank for the surrounding area' (Herrington and Lokman, 2016: 144). Thus, gardens become spaces of species becoming and movements. For Herrington and Lokman (2016: 143), what they call the growth of these '[m]igratory gardens', reflected in the designs of Clément, and the Dutch artist Herman de Vries, is the best strategy to engage 'the Anthropocene - an era that demands a rethinking of gardens as part of atmospheric, geologic, hydrologic, and biospheric changes ....

In the Age of Enlightenment, the dominant tropes were those of power and command, embodied in the design of famous gardens such as those of Versailles. But in today's 'Age of Entanglement' (Daou and Pérez-Ramos, 2016: 9), leading designers like Clément and de Vries are thinking with islands in order to reconceptualise design processes to better reflect the Anthropocene as a condition that we are all already in. As in the work of Tsing noted above, for Clément, gardens are patchwork islands of creativity, experimentation, and refiguration. ${ }^{4}$ Working with the disturbances and emergences of relational effects, illustrated by Patchwork thinking with islands, rather than in terms of bounded spaces of human control or the self-regulating harmony of Resilience, is seen as central for attuning designers to the conditions of the Anthropocene. 
Key here is the idea that ' $\mathrm{f}$ ] ar from suggesting a more hands-off design attitude, this actually requires a more intimate relationship between the designer and the designed' (Herrington and Lokman, 2016: 145). As in Tsing's analysis of satoyama practices, or Watts' (2018) work on Orkney, Patchwork approaches are far from laissez faire; on the contrary, they are deeply immersive, worlding practices. Again, it is work with islands which has played a notable role in bringing these concerns and the development of these approaches to the fore. For Libby Robin (2014), '[i]slands are a natural laboratory for science, they gave us evolution.' But, as she says, thinking with islands as important sites of ecological degradation in the Anthropocene in the ways of older, modern frameworks of separation and control, is not a model many contemporary conservationists embrace. Today, the space around island national parks is less likely to be understood as 'a biological desert' than a 'key to the success of the reserve. Animals use both surrounding landscapes and reserves in unexpected ways, and make ecological management more about watching and creating flexible responses to their needs, rather than demanding they follow human assumptions and building these into legislation' (Robin, 2014). A good illustration of this can be found in The Island with a Key to Our Future, where Selkirk (2020) examines the intensive Patchwork approaches being developed on Ascension Island; an exemplar of a 'novel ecosystem' approach where humans have purposely introduced and intensively managed non-native species on islands in order to increase biodiversification, with the result that the 'island's other native plants actually grew better because of the introduced species. ${ }^{5}$

The focus upon the relational entanglements and feedback effects of islands in Patchwork approaches brings out to great effect how the co-shaping of species or sympoiesis are understood as key characteristics of island life - something which aligns closely with Donna Haraway's (2008: 4) concept of 'figuration' which entered the analysis in the previous chapter, when referring to Laura Watts' (2018) work on the Orkney islands. ${ }^{6}$ Here, figures illustrate 'material-semiotic nodes or knots in which diverse bodies and 
meanings coshape one another' (Haraway, 2008: 4). As Haraway (2016: 56) says, it was the corals around islands which 'helped bring the Earthbound into consciousness of the Anthropocene in the first place.' It is the sympoietic feedback effects, capacities and affordances of island life which signify and draw out these new capacities from entities rather than an autopoietic process of self-development. Once it is clear that it is specific relational inter-connection that enables creative becomings, every relational nexus becomes a Patchwork island of potentiality, regardless of scale or fixed separations of time and space. Haraway powerfully reinforces the importance of this approach, arguing that ongoing processes cannot be grasped through homeostatic or autopoietic frameworks, which assume too many separations between entities, i.e. that relations are structured and limited. As she states:

The earth... is sympoietic, not autopoietic. Mortal worlds ... do not make themselves, no matter how complex and multileveled the systems ... Autopoietic systems are hugely interesting witness the history of cybernetics and information sciences; but they are not good models for living and dying worlds ... Poesis is symchthonic, sympoietic, always partnered all the way down, with no starting and subsequently interacting 'units'. (Haraway, 2016: 33)

For Patchwork ontologies, which work in a different way from the self-regulating approaches of Resilience, working with islands in the Anthropocene thus foregrounds a lively and unpredictable world of more-than-human relations. Phil Hayward's (2012a, 2012 b, 2018) work on the 'aquapelago' is another example of this. Hayward has drawn upon Suwa's above noted research into Japanese island cultures and shima, reading this alongside Jane Bennett's (2010) Vibrant Matter and his own research into Haida Gwaii (off the northern Pacific coast of Canada), to develop the aquapelago Patchwork ontology. Made up of islands, oceans, rivers and interweaving liquid relationalities in flux, for Hayward (2012b), the aquapelagos of Haida Gwaii draw our attention to a vibrant world that cannot be grasped by way of modern frameworks of reasoning (mind/body, subject/object and human/nature 
divides) or by Resilience analytics. For Hayward (2012b: 3), 'the humans who constitute aquapelagos through their engagements with terrestrial and aquatic spaces are (necessarily) ... characterised by the "vitality" of various non-human things.' They provide us with a rich relational ontology in which all entities, by existing or 'enduring', demonstrate an active persistence, a liveliness of 'conatus', having their own interests and effects. ${ }^{7}$ The aquapelago is a Patchwork ontology where relationality is revealed to be too rich and too complex to be reduced to a human/nature divide, or to be grasped or controlled by way of Resilience analytics.

As an island-oriented Patchwork relational ontology, Hayward's conceptualisation of the aquapelago has rapidly gained influence and informs a wide range of contemporary Anthropocene thinking - from research into monsoons in India (Bremner, 2016) to the geopolitical aesthetics of the subterranean processes of the emergence and disappearance of islands (Hawkins, 2018). ${ }^{8}$ Insightfully, Hayward links island studies scholarship itself to Anthropocene thinking more generally when explaining the development of his concept:

Aquapelagic relations are shifting and reconfiguring at rapid rates. The land areas, elevations and general viability of islands to support particular populations and their relationship to mobile expanses of waters and the nature of subsurface biomasses are in flux and require constant attention. To be an islander is, increasingly, to live in flux. To be an Island Studies scholar is, increasingly, to be scholar of flux. (Hayward, 2018)

To put this a different way, it is precisely because islands have long been understood as such key sites of relational entanglements and affordances in the wider sciences, social sciences, and in disciplines such as anthropology, that they are so useful for wider Anthropocene thinking today. It is the island as an important site of expansive relationality - and for the development of relational ontologies - which comes to the fore, and it is today heavily drawn upon for the development of Anthropocene thinking. As Haraway (2016: 57) astutely observes, '[w] hy is it that the 
epochal name of the Anthropos imposed itself at just the time when understandings and knowledge practices about and within symbiogenesis and sympoietics are wildly and wonderfully available and generative in all the humusities, including noncolonizing arts, sciences, and politics?' Her point, 'it matters which thoughts think thoughts' (Haraway, 2016: 57). It makes no sense to separate out social thought from the material world. We cannot separate out island imaginaries and contemporary broader trends in social and political thought from the material characteristics of islands as geographical forms which are doing important 'work' in such debates. Neither the Anthropocene nor islands exist purely 'out there' or purely 'in our heads'; rather, ontological statements made from working them - such as the relational ontologies or onto-epistemologies examined in this book - should be understood as objective facets of the given world itself (existing simultaneously in materiality and in thought) (Whitehead, 1967, 1968, 1985).

Thinking with islands for the development of what we are calling Patchwork ontologies and approaches - in this case with the aquapelago - recasts the world as rich and full of creative possibilities. Our appreciation of the liveliness of the world enables us to think more humbly about ourselves and our relation to the environment. Relations become less anthropocentric, narrow and instrumentalist compared to Resilience analytics. Instead, drawing upon the figure of the island and islanders, they are seen to become sympathetic, symbiotic and sympoietic, as we realise that we are not separate from the world but are interdependent with other nonhuman forms of life which we cannot grasp or control in the ways imagined by modernity. Working with islands in this way is thus generative for such Anthropocene thinking, where the aquapelago is one such example of:

... an 'onto-tale' in which everything is interacting ... it is the multiplicity of submarine depths, of regions of water and currents, of seafloor surfaces and their interactions with topologies of land and of aerial and weather systems, and of flows of 
materials between them, that produces an aquapelago ... (Hayward, 2012b: 12)

We agree with Stephanie Wakefield (2018:7) when she argues that today's ' $c$ ] ritical thinkers almost unanimously portray the infrastructures - and promises - of modernity with scorn or as ruins themselves (to think otherwise, the current discourse suggests, would be out-of-touch with the times - and perhaps worse, eliding or erasing the true nature of the world).' Patchwork ontologies, such as those developed by Hayward, Clément, and Tsing discussed above, productively work with island disruptions or perturbations, which may enable us to see new attributes, affordances and relations, and develop our own responsivities in 'learning to be affected' (Latour, 2004: 205). Instead of stories of Resilience, with their self-regulating and harmonious systems which seek to prevent or slow climate change, preserving the status quo, a Patchwork ontology approach leads to a different set of, much more affirmative, assumptions and practices engaging with the present in ways which are more open and creative, rather than merely adaptively responsive:

Staying with the trouble does not require such a relationship to times called the future. In fact, staying with the trouble requires learning to be truly present, not as a vanishing pivot between awful and edenic pasts and apocalyptic or salvific futures, but as mortal critters entwined in myriad unfinished configurations of places, times, matters, meanings. (Haraway, 2016: 1)

\section{Island Ontology as the New Ontology of the World}

To return to Haraway's (2016: 57) point, that it matters a great deal 'which thoughts think thoughts', it is useful to continue to mark the difference between Patchwork and Resilience ontologies of relation, in order to draw out what is at stake in these distinctions. The world of Resilience is one in which working with islands 
enables us to see the world as an immanent world in dynamic inter-relation, but the world is imagined as amenable to understanding and seen to be 'there for us', such that we are required to adapt to emergent effects by increasing our understanding of processes of interaction. The world of Resilience is one of increasing transparency, a world of adding agencies and attributes due to our appreciation of interactive interdependencies. But, as we have seen above, relationality is grasped differently in the approach of Patchworks; the intensity of relational interactions makes the world increasingly immune to human understanding and adaptive forms of governance. In a Patchwork world, we become aware that the world's liveliness and diversity are increasingly ungraspable, uncontrollable or incomprehensible as we ever plunge in and experiment anew. Thus, as we practice what it might mean to explore, journey or enter into the world in relation, another aspect comes to the fore in Patchwork ontologies: it is the opacity of the world, rather than its transparency, which matters.

In other words, Resilience approaches capture the experience of island being from the 'outside', as observers work to understand and adapt to the self-regulating nature of systems. Patchwork approaches, on the contrary, are analogous to being 'within' a world ontologically formed and reformed by islands as relational knots of time and space. Thus Patchwork practices reveal the complexity, richness and vitality of the world, as if on a journey which we cannot grasp from some external positional view or standpoint. The experience is ontologically quite different to that of Resilience. This matters for the development of thought in debates about the Anthropocene. In Resilience analytics, we are adding a new way of understanding and explaining difference, providing alternative possibilities or choices for instrumental application. In Patchwork approaches - such as those of Tsing, the new experimental designers, and Hayward's aquapelago above - we are not discovering the world but always in the process of making it, through practices of relation. Resilience approaches separate us as subjects from the world, adding to our knowledge about it as external systems or processes; Patchwork approaches bring us back into the world, 
not as knowing or separated subjects but as creative co-creators in multiple 'islands' of relational entanglement.

As we said in Chapter 1, island thinker Édouard Glissant intimates what is at stake in distinguishing Resilience and Patchworks approaches to relational ontology. In Poetics of Relation (1997), Glissant articulates an island analytic to highlight the limits of 'reductionism' in much Western thinking. He argues that Einstein's theory of relativity does not take relational ontology far enough and thereby 'is not purely relative' (Glissant, 1997: 134). For Einstein, " $t \mathrm{t}]$ he universe has a "sense" that is neither chance nor necessity', this provides "'guarantees" [both of] the interactive dynamics of the universe and of our knowledge of it' (Glissant, 1997: 134). Thereby: 'Just as Relativity in the end postulated a Harmony to the universe, cultural relativism (Relativity's timid and faltering reflection) viewed and organised the world through a global transparency that was, in the last analysis, reductive' (Glissant, 1997: 135). Glissant is here highlighting that relationality provided different viewpoints or standpoints which still located the human as external to the world of relation, still managing and directing the powers of immanence, understood as amenable to universal laws and regularities.

Thus, for Glissant, as for our heuristic framework of relational ontology developed throughout the first half of this book, there are two 'tendencies' or key locus points in a continuum within ontological approaches of relational becoming, both of which appear in contemporary thinking drawn from island experiences and practices. The first approach to working with islands, that we analyse as Resilience, appeals to scientific, evolutionary, or underlying cybernetic laws and rationalities of 'interactive life' and 'has become increasingly based on attempts to imagine or to prove a "creation of the world" (the Big Bang), which has always been the "basis" of the scientific project' (Glissant, 1997: 136-137), enabling a Darwinian evolutionary telos of progress and increasingly complex differentiation. Despite claims often to the contrary, nevertheless, 'The idea of God is there. And the notion of legitimacy reemerges. A science of conquerors who scorn or fear limits; a 
science of conquest' (Glissant, 1997: 137). The second approach to relation though, tends in:

... the other direction, which is not one, distances itself entirely from the thought of conquest; it is an experimental meditation (a follow-through) of the process of relation, at work in reality, among the elements (whether primary or not) that weave its combinations... This 'orientation' then leads to following through whatever is dynamic, the relational, the chaotic - anything fluid and various and moreover uncertain (that is, ungraspable) yet fundamental in every instance and quite likely full of instances of invariance. (Glissant, 1997: 137)

Thus, Glissant (1997: 142) advocates an alternative science of poetics, which seeks to dig deeper into the world through 'givingon-and-with', challenging universal, generalising or transcendent totalities in its focus on ever 'more stringent demands for specificity. His approach is a practical one, like that of Tsing, Hayward or the designers noted above, in which the subject is no longer an observer of relations but practically worlding itself in a concrete embedded and embodied way. ${ }^{9}$

For Glissant, then, there are two ways in which relational, interactive or processual ontologies can be related to, and the stakes involved make a major difference. Another way in which he expresses this is in the binary pairing: 'thought of the Other' and 'the other of Thought'. For the former, there is a moral generosity and an appreciation of alterity as new forms of knowing and adapting are enabled as the world to be governed expands (as in the discourses of Resilience). For the latter, the world opens to experience in 'an aesthetics of turbulence whose corresponding ethics is not provided in advance' (Glissant, 1997: 155). Thereby, the 'other of Thought' is an onto-ethical practice: 'the work I am to undertake, the road I am to travel' in order to contribute to and 'to join the dynamics' (Glissant, 1997: 155). This is the ontology of Patchwork approaches, which Glissant foregrounds, or prophesises, where to undertake island work is to make a difference not by discovering something or contributing to a universal store of 
knowledge, but by 'joining the dynamics', taking the immersive plunge and 'staying with the trouble'.

For Roberts and Stephens (2017: 19), engaging Glissant's thinking with islands in this way facilitates an 'anti-explorer' method, a feminist approach that challenges the idea of the (White, Modern male) explorer who 'sallies forth with confidence that if the world is as yet unknown, then it at least may be surveyed and hence known via Euclidean geometry' (Roberts and Stephens, 2017: 20). In Glissant, this is articulated through 'the infinite island' (Roberts and Stephens, 2017: 26) 'a maelstrom, a place constituted by infinitely large numbers of analytical frames moving toward the infinitely minute' (Roberts and Stephens, 2017: 28). This is not about boasting to modern, mainland thinking 'If you have the massive continent, then we have the infinite island' (Roberts and Stephens, 2017: 23, emphasis in original). Rather, it is to foreground how Glissant's thinking with islands is a practical one in which the subject no longer stands apart, outside or above as an observer of relations, but rather practically worlds themselves expanding their world - in embedded and embodied ways which cannot be known in advance. Thus, the anti-explorer method expands worlding into:

... those experiences of islands that have not or have yet to be integrated into our discourses, our measurements, our archives, and our tropes. These may be local, island knowledges, some of which are lost, contingently receding, or resurgent within the dominance of other epistemological frames, ranging from the most local use of an herb to the cosmic navigational worldview of Pacific Island canoers who have perceived the islands as moving in relation to the stars. (Roberts and Stephens, 2017:24)

For such Patchwork ontologists, islands are not understood as worlds that we are in, to be merely adapted to; they are ways of expressing and understanding our own processes of worldmaking. ${ }^{10}$ Mimi Sheller (2020) similarly examines how the Taino/Arawak and later Afro-Caribbean peasant gardening practices ofintercropping, silviculture, silvipasture, and conuco gardens, 
function as sites of inter-species relationality and Patchworks. In Patchwork approaches, it is the bringing into relation and awareness which matters and is key to generating creative outcomes or possibilities (see also Sheller, 2018; Mika, 2018). Patchwork approaches are not determined by the power of entities but the contingent effects of inter-relation. They draw upon islands and certain islander practices as processes of relating, of bringing together, and the capacities for attentiveness or attunement to effects generated through these relations. ${ }^{11}$

Godfrey Baldacchino and Eric Clark (2013: 131) have written in this way about islanding as world-making, where they quote the Pacific island poet and scholar Teresia Teaiwa: 'Shall we make “island” a verb?'. Teaiwa (2007: 514) continues:

As a noun, it's so vulnerable to impinging forces. Let us turn the energy of the island inside out. Let us 'island' the world! Let us teach the inhabitants of planet Earth how to behave as if we were all living on islands! ... The islanded must understand that to live long and well, they need to take care. Care for other humans, care for plants, animals; care for soil, care for water. Once islanded, humans are awakened from the stupor of continental fantasies. The islanded can choose to understand that there is nothing but more islands to look forward to. Continents do not exist, metaphysically speaking. It is islands all the way up, islands all the way down. Islands to the right of us, islands to the left... Yes, there is a sea of islands. And 'sea' can be a verb, just as 'ocean' becomes a verb of awesome possibility. But let us also make 'island' a verb. It is a way of living that could save our lives. ${ }^{12}$

For contemporary Anthropocene thinkers like Sheller (2020: 153) there is much to be gained from this way of 'islanding' the world:

Teaiwa presciently moves toward a new horizon for thinking through 'islanding' as a positive healing practice that holds out hope not just for [islands like] Haiti, the Caribbean, or the Pacific, but for humanity as a global archipelago. If we can all be islanded, become islanders, and do islanding, then we can perhaps learn to reject the forms of violence and ecocide that we have been 
inculcated in, and to better cooperate in making a more just Island Earth.

For us, thinking 'island' as a verb, a process of world-making, rather than a static noun, has been informed by Deborah Bird Rose (2017b: 34) on how the Aboriginal islanders of Australia 'have picked up the English word "country" and used it discursively; they have absolutely run away with it. For these islanders, the term 'country':

... is multi-dimensional: it consists of people, animals, plants, Dreamings, the dead and the yet to be born, underground, earth, soils, minerals and waters, surface water and air. There is sea Country and land Country; sky Country too. Country has origins and a future; it exists both in and through time ... The sites of Dreamings' or creators' actions are in Country, and their tracks criss-cross Countries, connecting one to another through the great songlines or travels that were at the origin, and now are at the centre, of the on-going-ness... So Country is not 'ours' as the government says in its literature on 'Caring for the Country', as if it were some sort of entitlement or as if we were the boss. Country is an intergenerational, interspecies gift of life. (Bird Rose, 2017b: 34-35, 41)

Tsing (2015: 23) calls this open-ended process, of collective and connective experimentation, 'ways of being', understood as 'emergent effects of encounters': the possibilities inherent in fluid assemblages with others. ${ }^{13}$ In life after modernist dreams of progress, disturbances and perturbations - thinking with islands as verbs rather than reductive nouns - is not a threat to the status quo but an interactive invitation to creativity, seen as a positive opportunity to make 'life in capitalist ruins':

Making worlds is not limited to humans. We know that beavers reshape streams as they make dams, canals, and lodges; in fact, all organisms make ecological living places, altering earth, air, and water... In the process, each organism changes everyone's world. Bacteria made our oxygen atmosphere, and plants help maintain it. Plants live on land because fungi made soil by digesting rocks. 
As these examples suggest, world-making projects can overlap, allowing room for more than one species. (Tsing, 2015: 22)

Thus, as noted, drawing upon her research into Japanese islander practices, Tsing (2015: 258) tells the story of woodland revitalisation groups 'who hope that small-scale disturbances might draw both people and forests out of alienation, building a world of overlapping lifeways in which mutualistic transformation, the mode of mycorrhiza, might yet be possible.' She states, 'They hope their actions might stimulate a latent commons, that is, an eruption of shared assembly, even as they know they can't actually make a commons' (Tsing, 2015: 258; emphasis in original). Patchworking ontology informs a set of techniques not so much for 'making' something but rather as a creative stimulus; cultivating, exploring, probing, facilitating, repurposing and amplifying what already potentially exists, but which can only come into being 'with'. The new potentialities for thinking with island ontologies in this process of world-making rely not upon latent essences within a preexisting entity but in the interactive creation of a new 'commons' and have come to be associated with a duty or ethics of care for living in the Anthropocene as a condition in which we are all already in.

\section{Patchworks as a Duty of Care: Amplification and Attentiveness}

Patchwork thinking with islands is thus productive, and nowhere is this more obvious than in the work of Glissant. John Drabinski (2019: 46, emphasis in original) has recently underscored how the geographical specificity and materiality of the Caribbean islands were central to the development of Glissant's approach:

Glissant's literary and theoretical work consistently engages with the image and botanical-geographic meaning of the mangrove in order to characterize the poly-rooted, rhizomic character of Antillanité ... The tropical mangrove, the Rhizophora, survives 
precisely because it is lifted above the sea with special roots. Propped above the tidal pulse, the tree's body is given oxygen, which keeps it alive despite the unpredictable and often violent crashing of salt, time, memory, and waves on the shoreline. Yet, the mangrove is also constantly contested by the sea. Becoming mangrove is no easy piece. It survives because the roots have lifted what is essential above the contingent destructive reach of the waves. The mangrove is a border plant. Roots like no other - plural and in no relation of dependency to the One. Difference without identity, yet an identity. The botanical archipelago, the repeating island bordering the island that repeats. Death and life intertwined without melancholy. The fecundity of the Rhizophora. ${ }^{14}$

Glissant, explicitly thinking with Caribbean shorelines, provides a very different, more productive, way of engaging the ruins of modernity than that of Walter Benjamin's famous treatment of 'allegory, ruin, and history' (Drabinski, 2019: 66) - there is thus a 'critical chasm between Benjamin's Europeand Glissant's Caribbean in terms of the structure and meaning of historical experience' (Drabinski, 2019: 68). Whilst Benjamin focused upon how allegory is employed in such a way which makes it difficult to 'make any distinction between pain, history, and memory' (Drabinski, 2019: 68), foremost in Glissant's work is how 'history gathers itself into the ruins of landscape and language' (Drabinski, 2019: 68). Glissant's 'Caribbean historical narrative sets out, not from Benjamin's analogy of allegory, but from the abyss [of the Middle Passage] in order to arrive at place and the peculiar mixture of times and spaces that comprise the nonlinear constitution of beginning' (Drabinski, 2019: 78). This was, of course, not only Glissant's, but Derek Walcott's 'project of the storyteller' - a question of 'becoming Caribbean through the reading of ruins' (Drabinski, 2019: 71, emphasis in original); thus, demanding the work and labour of the islander's own processes of world-making:

Ruins are fragments, yes, but ruins and fragments are also always in need of the poetic work of bringing forth, forming and reforming, and so a kind of beauty-making memory project that loves 
the past as much as the future. Love reassembles fragments. [As Walcott says in his Nobel Prize-winning speech. ${ }^{15}$ ] The sigh becomes the poet's fidelity to possibility. (Drabinski, 2019: 71)

This is nothing less than an alternative worldview to Benjamin's European perspective of the ruins of modernity. For Glissant, like Walcott, 'Thinking ruins, which is productive rather than (solely) melancholic, is already thinking the archipelago as a geography of the globe and the geography of thought. The archipelago is already the crossroads of the world, so the Caribbean and Caribbeanness is already tout-monde in memory, history, and experience ...' (Drabinski, 2019: x). Patchwork ontologies are thus stories of cultivation rather than extraction or melancholy; an ethico-political duty of care that is situated fully in the present.

The Patchwork approach of cultivation and care is exemplified in the work of María Puig de la Bellacasa. Drawing upon her experience of permaculture training, she states:

Obligations of caring in naturecultures cannot be reduced to 'stewardship' or 'pastoral' care in which humans are in charge of natural worlds. Such conceptions continue to separate a human 'moral' subject from a naturalized 'object' of caring. Nor need we go to the other extreme: diluting the thinking of specific obligations of care in situational relations with nonhumans ... These are poor generalizations that avoid engaging with actual situated naturecultures and the speculative efforts demanded from ecological thought and practice. (Puig de la Bellacasa, 2017: 164, emphasis in original)

For Puig de la Bellacasa (2017: 165), if discourses of Resilience are a biopolitics then the Patchwork ontologies examined in this chapter are an 'alterbiopolitics'; creating different forces of worldmaking relationalities, capable of cultivating " power with" and "power-from-within" rather than "power-over". Patchworks are not only sensitive to feedbacks and unintended effects; Patchwork approaches hold unexpected possibilities for creative experimentation in the Anthropocene, understood as a condition we exist 
within. Patchwork ontologies are not necessarily against technological applications, any more than those of Resilience, but seek to apply them differently. In Patchwork ontologies, technologies are experimental ways of bringing out relational capacities rather than constraining them (Viveiros de Castro and Danowski, 2018: 187).

A good example of this is 'Hubs in a Sea of Knowledge: The Startling Adventures of RonR', presented at the 2018 International Small Island Studies Association (ISISA) conference by the Wetlands Wanderers (comprising Jan de Graaf and Jeroen van Western). Creatively bringing together expertise in science, art and engineering, as well as inviting members of the general public into the process, 'The Startling Adventures of RonR' recounts the story of hacking (with permission) into bird-tracking devices, in order to expand awareness of and care for Terschelling Island's (Netherlands) rich and multidimensional relations; and, in the process, problematise the all-too-coherently drawn boundaries of the Wadden Sea UNESCO World Heritage Site. Doing this, the Wetlands Wanderers (2018) draw attention to:

... how migratory birds know more than the average wellinformed citizen of the world. They fly over dangerous places, on their way to strange places where coarse languages are spoken. Their map is a map of the world. Sometimes they observe things that should stay hidden, and we are left wondering what they've seen. The bird's eye view implies a certain distance to the object of perception. Birds inspire a curiosity in us because they are windows to the world ... The Wadden Sea is part of the North Sea, which in turn is part of the Atlantic Ocean. Lines intersecting the Wadden Sea draw our gaze north to the polar regions, and south to well beyond the equator.

'The Startling Adventures of RonR' tracks the complexity of bird movements into the North Sea, Atlantic, Canada, Africa and Russia. It richly expands island relations into cloud, atmospheric, oceanic, terrestrial and a range of other entanglements; incorporating both expert and public engagement in processes of surveying, 
scanning, listening, tasting, looking around, observing weather, writing history, navigating, bird-watching, spying and imaging. This is a much more expansive, Patchwork approach than merely correlating the patterns. As the project designers say, it is:

$\ldots$ an exercise in observing, a training in sensory perception and awareness. To watch, listen, taste and identify. We count - and we recount stories, operating on the brink of fact and fiction. We do light-hearted science and experimental philosophy. (Wetlands Wanderers, 2018)

Importantly, unlike Resilience responses to adaptation which assume beforehand the correlations and changes to be modulated to maintain equilibrium, such Patchwork island analytics make no assumptions about the meaning or consequences of signs. Thus, as in the case of previous examples discussed, the process of attentivity, attunement or 'affectedness' is much greater and more intense. It is this process of Patchworking which 'expands the present' and cares for the future, literally bringing the future into being by responding through close attention to feedbacks. But unlike the more instrumentalising approach of Resilience, every sign, signal or change in the state of being provides an 'opportunity' to bring new futures into being and demands to be 'seized' rather than 'wasted.' ${ }^{16}$ In counterposition to a Resilience analytic which subsumes islands under the power of the world, this form of interpretation goes beyond modernist distinctions of self and other as 'there is no illusion of transcendence or transparency' (Marques, 2017: 34). Thus, Patchwork approaches, such as those exemplified by the Wetlands Wanderers, enable us to:

... rupture the hegemonic gaze which sees objectivity everywhere. To think images as the embodiment of worlds means not only thinking the ontology of images but also thinking images ontologically, that is, not as representations but as representatives: ... images through which we see other images. (Marques, 2017: 37)

The key point is that in Patchworking approaches, such as those adopted in 'The Startling Adventures of RonR', or Tsing, or Bird 
Rose, signs or signals are held to enlarge the world of possibilities and of potentials rather than subtracting from or limiting it. Here island ontology opens up as the ontology of the world: a process of world-making which extends and expands in space and time as a way of enlarging the present through a more open, less reductive, ethos or duty of care.

We can further see this process of thinking with islands as worldmaking and an ethos or duty of care in the work of the highly acclaimed poet Juliana Spahr, who writes about Hawai'i. In This Connection of Everyone with Lungs, Spahr (2005: 9) slows down her pulse in order to breath in, bring into consciousness, amplify and attune to the vastness of island relations in her own inimitable rhythmic style of writing:

The entering in and out of the space of the mesosphere in the entering in and out of the space of the stratosphere in the entering in and out of the space of the troposphere in the entering in and out of the space of the oceans in the entering in and out of the space of the continents and islands in the entering in and out of the space of the nations in the entering in and out of the space of the regions in the entering in and out of the space of the cities in the entering in and out of the space of the neighborhoods nearby in the entering in and out of the space of the building in the entering in and out of the space of the room in the entering in and out of the space around the hands in the entering in and out of the space between the hands.

In these Patchwork approaches island relations stretch out massively in space and time, and any attempt to grasp and control them in the way of modern frameworks of reasoning, or the analytics of Resilience, simply slips through the fingers. On the one hand, there is a dizzying sense of being overwhelmed and the desire, which is continuously thwarted, to take everything in. On the other hand, the slowing down of the pulse, the body, the breathing, 'the entering in and out', works to amplify what already exists, bringing it into awareness via processes of attunement. ${ }^{17}$

Boaventura de Sousa Santos (2016: 186) provides an extremely useful and informative philosophical framing, with his two con- 
ceptions of a 'sociology of absences' and a 'sociology of emergences' which can be read as a how-to guide for working with a Patchwork ontology in order to develop an ethics or duty of care. The 'sociology of absences' is designed to make the everyday unusual so that we can pay attention to it, thus 'expanding our available realm of experiences'. We can then see more 'signs or clues', thus in working with islands' relational entanglements, disturbances and emergent effects, our world becomes stranger to us. The 'sociology of emergences' then expands this speculative moment 'decelerating the present, giving it a denser, more substantive content', enabling 'ethical vigilance over the unfolding of possibilities' aided by such emotions as (negative) anxiety or (positive) hope. Together this method provides what de Sousa Santos (2016: 186) calls 'symbolic amplification.'

Juliana Spahr's Hawaiian poetry is a particularly good illustration of this 'symbolic amplification'. So is the work of Deborah Bird Rose (2017a: G53), who draws upon the conceptualisation of 'shimmer', an Australian Aboriginal aesthetic, to discuss the ways that signs and signals 'appeal to the senses, things that evoke or capture feelings and responses ... lures that both entice one's attention and offer rewards.' As Bird Rose (2017a: G54) explains, shimmer pervades many aspects of Aboriginal island life, for example:

At an ecological scale in northern Australia, one of the most obvious patterns is the pulse between wet and dry seasons. The desiccation of the dry season dulls the landscape in many ways (although the country is always beautiful): there is a winding back of fertility, a loss of water, and thus loss of the possibility for sun to glint on the water. But then, things begin to move toward brilliant again: the lightning starts to spark things up, the rain starts to bring forth shiny green shoots, and rainbows offer their own kind of brilliance. Shimmer comes with new growth, the everything-coming-new process of shininess and health, and the new generations.

Shimmer is a form of expansive amplification of the richness and complexity of island relations, which does not understand the 
world as being 'composed of gears and cogs but of multifaceted, multispecies relations and pulses' (Bird Rose, 2017a: G55). For Bird Rose (2017a: G55), only in this way can we bring out the full potentiality of (island) life; its 'diversity, complexity, abundance, and beauty'. Rather than a universal theory of progress, where the past was always a necessary moment, fixing the determination of the present, for such Patchwork ontologies, the past is an 'inexhaustible' resource for holding open transformative hope in the present and for an ethics of care: '[f] or shimmer to capture the eye, there must be absence of shimmer. To understand how absence brings forth, it must be understood not as lack but as potential' (Bird Rose, 2017a: G54). This is why there is a need to expand thinking with islands into a focus upon the richness and depth of relation as potential and possibility.

For Patchwork ontologies, the world is always necessarily more than its surface appearance. This is why 'symbolic amplification' is necessary in such works as 'The Startling Adventures of RonR' about Terschelling Island's expansive relational entanglements, the Hawaiian poetry of Spahr, and the research of Bird Rose with Australian Aborigines - to work with islands is to see beyond the limits of modern modes of thought. What does not appear to exist, or is not readily apparent, may be important and rich in potential. We have also seen this, as noted above, in, for example, Glissant's (1997) 'the Other of thought', Roberts and Stephens' (2017) 'antiexplorer' method and Hayward's (2012a; 2012b; 2018) 'aquapelago': all approaches developed by working with islands. This is why thinking with islands as significant figures of relational entanglements and awareness in the Anthropocene has become important for contemporary commentators, and it is what gives the analytics of Patchworks its agential and futural appeal. As Bird Rose (2011: 114) argues: 'Part of what makes our common Earth condition so interesting is that that which may yet be is infinitely more extravagant than that which already has been.' Uncertainty or unknowability do not close down our world but open it up as 'the possibilities of the living world always are greater than the mind or knowledge system that wants to understand' them (Bird 
Rose, 2011: 114). The 'not yet' and the 'may yet be' are here and not here at the same time and thus the purpose is not to reproduce or conserve the present but 'to enable,' 'to engender', 'to cultivate', or 'to care' futurally.

While Resilience ontologies work with islands by paying attention to systemic interaction, feedback effects and to tipping points, for Patchwork ontologists such approaches would inevitably be productionist, consumptionist and extractivist. Resilience analytics are always focused on saving, or on prolonging, or making more efficient what already exists. In the Anthropocene, these approaches stand accused of refusing to see that these contemporary forms of being are, themselves, the problem. The alternative ontological islands lens which promises change and transformation is that of Patchworking, which can be learned by working with islands and island peoples and cultures. This approach trains us to attend to the world around us, enabling us to develop skills giving 'symbolic amplification' to the clues and signs all around us. We believe it matters that the examples discussed in this chapter have been explicitly developed from work on islands. This attentiveness, cultivated by thinking with islands as important sites of relational entanglements, can be transformatory, expanding our reality beyond modernist constrictions and making available infinitely more possible, concrete futures (de Sousa Santos, 2016).

\section{Conclusion}

The key premise of this book is that working with islands helps to engender and to clarify the core methodological and conceptual frameworks for Anthropocene thinking today. Thus, whilst many commentators would perhaps choose to focus upon how Patchwork ontologies could be aligned with, say, the work of Deleuze and Guattari's (1986) assemblage theory, ${ }^{18}$ this would tell us little about the particular geographical forms and cultures which are doing the 'work' in Anthropocene thinking. We think this is something which has been missing from contemporary debates, which we will pick up and develop in more detail in the concluding 
chapter of the book, turning to a critical agenda for island studies in the Anthropocene. For now, we want to make this simple claim: it matters that contemporary experiments in governance, design and theorising regularly engage islands and islanders in order to aid and develop their thinking.

This chapter has focused upon the emergence of what we call 'Patchwork ontologies' in Anthropocene thinking; which, like Resilience analytics, draw heavily upon islands as sites of relational entanglements and feedback effects. But whereas Resilience engages islands in terms of self-regulating systems and cultures which tend towards organisational harmony and adaptation, Patchwork approaches 'stay with the trouble', where working with islands foregrounds a world which is too lively, too complex, and too unpredictable to be grasped by modern frameworks of reasoning. Patchwork ontologies, widely prevalent in experimental approaches to Anthropology, design, the arts, technological experiments, poetry, and ethnography, thus think with ongoing disturbances and emergent relational effects. Developing and drawing upon particular ways of thinking with islands, this becomes a way of expressing and understanding our own processes of worldmaking; which, for Patchwork ontologies, is often associated with a duty or ethos of care.

In bringing this first half of the book to a close, we suggest that Resilience and Patchwork relational ontologies are two important ways of understanding how thinking with and from islands has influenced wider Anthropocene thinking. The figures of the island and islander are not peripheral in these developments, but have an important place on the international stage, as we have seen in the wide range of examples above. Islands are both important in terms of being the key symbols of transforming planetary conditions, and in terms of the increasing attention given to non-modern, relational entanglements and ontologies in debates about the Anthropocene. As we said in the introductory chapter, readers may think of other ways in which work with islands in the Anthropocene can be analytically and heuristically clarified to help us better understand and contribute to contemporary debates; 
and, indeed, we very much hope that they will. We see this book as a beginning, exploring some avenues for engaging the work that islands do in shaping contemporary Anthropocene thinking more generally. Whilst the first half of the book has focused upon what we understand to be two dominant approaches to relational ontology, the second half turns to the analysis of two contrasting approaches to onto-epistemology, both strongly associated with thinking with islands in the Anthropocene; what we call 'Correlation' and 'Storiation'.

\section{Notes}

${ }^{1}$ For example, in art and island culture, as Hsinya Huang (2017) points out, Bill Reid's sculpture The Spirit of Haida Gwaii, the Black Canoe of an Indigenous canoe from the Pacific Northwest of the Americas, is an ecological metaphor of a lifeboat during the great flood. Centrally, the inclusion of many different species on the boat is said to foreground 'the restored continuum of human and nonhuman beings in ecological peril, and retrieves a multispecies eco-aesthetics rooted in Indigenous stories and myths of the Pacific' (Huang, 2017: 286).

${ }^{2}$ As Lovelock (2020: 12) recently affirmed, 'In Greek mythology, Gaia is the Greek Goddess of the Earth and, at the suggestion of the novelist Willian Golding, I gave her name to the theory I developed fifty years ago. The theory is that, since it began, life has worked to modify its environment.'

${ }^{3}$ For example, where a geographical form like the desert is engaged this is more frequently envisaged as a blank space for the projection of modernity, rather than as a productive space for thinking through relational entanglements (Günel, 2019). Compare this to commonplace statements about islands, which are today widely understood as 'particularly potent landforms for a reimagination of the earth and our relation to it, which is partly due to the imaginative potential of their geo(morpho)logical instability (think, for instance, of volcanic islands). Indeed, if islands lend themselves to a discussion of productive processes, they can equally be mobilized to negotiate destruction and dissolution' (Riquet, 2020: 4). As noted throughout this book, this perspective is commonplace in the literature when it comes to both islands and island cultures. As another example which reflects 
contemporary Patchwork approaches more specifically, Katie Ritson (2020: 10) discusses how 'The portrayal of northern Atlantic subsistence cultures is an imagination of a different kind of past, one that existed simultaneously with but outside the mainstream narrative of capitalist progress and industrialisation; it creates an open-ended space where the imagination of possible futures can be explored.'

${ }^{4}$ Similarly, for Grove et al (2019), commenting on design practices more generally: 'Recent decades have seen design shifting its concern from objects to processes, systems and futures. Design orients thought and action not towards questions of how something came to be, but rather what something might become, crafting new futures from within, rather than outside, the present.'

${ }^{5}$ Thus, debate has moved on significantly when thinking with islands in the Anthropocene. Compare the Patchwork, more open-ended approaches of becoming, examined by Robin (2014), Herrington and Lokman (2016) and Selkirk (2020) above, to older approaches where it was argued that 'immigration, which is important in maintaining species equilibrium on true islands, will not contribute significantly to the maintenance of equilibrium on reserves in the future because of the disappearance of recolonisation sources' (Pickett and Thompson, 1978: 27).

${ }^{6}$ As Crane and Fletcher (2017) further point out, Ursula K. Le Guin, one of the central influences upon Haraway, also thinks with islands in this way. Focusing upon a world of island and archipelagic relations, with no continents, Le Guin's Earthsea series 'eschews the closed system narrativization of so many island genre fictions' (Crane and Fletcher, 2017: 161) in favour of a Patchwork approach, foregrounding a world of islands of creativity, emergent disturbances and effects.

${ }^{7}$ See also Kelly and Lobo's (2020) work with tidal country and cultures in North Australia.

8 Thus, Hayward's work has been widely drawn upon in the journal Shima to develop 'a form of collective 'thinking with islands' to understand contemporary phenomena with implications for other natural-cultural systems. [For example, working] at the intersection of Anthropocene studies, cultures of infrastructure, and tourism studies on islands and aquapelagos across the globe' (Moore, 2020: 2).

9 Thus, Gökçe Günel, Saiba Varma and Chika Watanabe (2020) term a 'Patchwork ethnography' as one that 'does not react to the externalities of the world by demanding more productivity. Instead, it seeks 
to remake that world by erasing pre-given categories and boundaries between our personal and professional lives.'

${ }^{10}$ Such an approach is highly influential in contemporary work. Much of Katherine McKittrick's (2006: 128) Demonic Grounds: Black Women and the Cartographies of Struggle is centred upon literal and metaphorical island imaginaries which enable us to think about how the production of space is worked out through mapping and attempting to constitute the space of human Others as disembodied and then transparently abnormal' (see also Roberts, 2020). McKittrick (2006: 129) engages the work of Caribbean island scholar, Sylvia Wynter, to draw attention to how, through colonialism and slavery, the islands of the 'New World' became places where 'the uninhabitable was abstracted by cartographic translations of where and who can constitute the terms of normal habitability'. The extension of this logic manifests today in what McKittrick (2006: 133) calls 'archipelagos of poverty', from North American prisons to the islands of the Caribbean. The counter-move, for McKittrick, is to follow Glissant, in drawing out the "real but long unnoticed" places of interhuman exchanges: cultural sharings, new poetics, new ways of being, "a new world view"' (quoted in McKittrick, 2006: 132):

These encounters always include the under-represented conceptions of being in place - the spaces of Otherness, subjective worldviews - that may not be immediately available in our geographic imaginations because Mans' sense of place is naturalized as normal. However, archipelagos of poverty, hemmed in and categorized by global color-lines and biocentric logics - are, like Man's geographies, inhabited. And, if we return to Glissant and connect his poetics of landscape to this present discussion, encountering, saying, and living geography brings this present subject into being ... spaces of Otherness are 'palpitating with life'. (McKittrick, 2006: 132-133).

${ }^{11}$ For island scholars, such approaches have a longer lineage in Hau'ofa's (2008) invocation to think in terms of a 'world of islands' (rather than, in the modern or mainland sense, 'islands of the world'). For Hau'ofa (2008), the island cultures of Oceania have a different worldview from continental and mainland thinking. They do not think in terms of parts or wholes but rather through interactions and relations. As 
Rakuita (2017) explains, relations are not reduced to intermediaries between autonomous entities, but neither are entities constituted or fully determined by their relations. Rather, a 'world of islands', while contingent and porous, is generative of effects: i.e. (island) life is not determined by the power of autonomous entities but the effects of inter-relation. To quote Hau 'ofa (2008: 32-33): “'Oceania” denotes a sea of islands with their inhabitants. The world of our ancestors was a large sea full of places to explore, to make their homes in, to breed generations of seafarers like themselves. People raised in this environment were at home with the sea. They played in it as soon as they could walk steadily, they worked in it, they fought on it. They developed great skills for navigating their waters - as well as the spirit to traverse even the few large gaps that separated their island groups ... Theirs was a large world in which people and cultures moved and mingled, unhindered by boundaries of the kind erected much later by imperial powers.' (See also Jolly, 2007).

${ }^{12}$ The notion of thinking with islands as a verb is today widespread in many Patchwork approaches. As another example, thinking with the islands of the Caribbean and with the work of Glissant, for Yountae (2016: 137-138, emphasis in original): 'the common world is not something to be "discovered", or taken for granted. The ground ... is not a noun, a mere description of the given ground, but a verb, an action in process, a process in action. It grounds. It refuses, therefore, to be a mere description of the cosmopolitan state of globalized capital or the elitist ideal of neonomadic transnationalism accompanying it... [Rather, it] begins from the ruins, from below, by cocreating the world ... Its making is also its unmaking in that it is an open project, always becoming, always creolized.'

${ }_{13}$ There are many contemporary examples of thinking with islands as world-making, in this way; from Emanuela Borgnino's (2020) work in Hawai' i, to Flores and Stephens (2017) 'relational undercurrents', to the powerful poetry of Perez (2020b) from Guåhan (Guam), and Pippa Marland's (2014) work on Wales' Bardsey Island.

${ }^{14}$ In drawing out how Patchwork and Resilience relational ontologies exist on a spectrum, or sliding scale, we can compare Glissant's Patchwork approach to thinking with mangroves and the Resilience thinking with mangroves examined in Sahana Ghosh's (2020) article 'How Rhizophora mangroves on Car Nicobar Islands fought back a rapid sea-level rise in 2004 tsunami'. As noted, Glissant focuses upon how mangroves have roots which are plural, in no relation to the 
One. Thus, disturbances and emergent effects open out to much less predictive, more generative or creative possibilities, which cannot be reduced to linear space-time imaginaries. This is whilst, in Resilience thinking with mangroves, the focus is more upon how mangroves facilitate islands to 'bounce back' after disasters - protecting 'shorelines from eroding and shield[ing] communities from floods, hurricanes, and storms' (Ghosh, 2020). Thus, the focus is upon how mangroves exist within closed-loops circuits of 'adaptability' which allow them 'to thrive in habitats that experience a long duration of flooding by seawater' (Ghosh, 2020). Whilst both Resilience and Patchworks are non-modern relational ontologies, Resilience adopts a more linear, or fixed, understanding of space-time.

15 'Break a vase, and the love that reassembles the fragments is stronger than that love which took its symmetry for granted when it was whole. The glue that fits the pieces is the sealing of its original shape. It is such a love that reassembles our African and Asiatic fragments, the cracked heirlooms whose restoration shows its white scars. This gathering of broken pieces is the care and the pain of the Antilles, and if the pieces are disparate, ill-fitting, they contain more pain than their original sculpture, those icons and sacred vessels taken for granted in their ancestral places. Antillean art is this restoration of our shattered histories, our shards of vocabulary, our archipelago becoming synonym for pieces broken off from the original continent' (Walcott, 1998: 69).

${ }^{16}$ See also, for example, Sound of Mull, which is 'a series of performance scores developed through artistic practice-as-research into how to perform geochronology in the Anthropocene' (Rawlings, n.d.) on the island of Mull. The book Sound of Mull, by Angela Rawlings (2020), contains a range of instructions from how to listen to deep time to how to knit plastic collected from shorelines.

${ }^{17}$ As Nicole Merola (2018:43) says, 'It is not enough, Spahr's work argues, to merely represent or think the affects of the Anthropocene. Rather we have to performatively embody them in ways that materialize our vulnerabilities, whether shared or particular. Spahr's continued formal experiments and her activisms around constructing literary communities help us practice forms of Anthropocene inhabitation. While these activities will not assure survivability, they do operate as critical coping mechanisms that register and compose how we conceptualize and live in the Anthropocene, its effects, and its affects.' We find a 
similar approach in the work of Nadim Samman and Julian Charrière (2018: 138-139), who, diving into the waters of Bikini Atoll, the most famous nuclear testing grounds in the world, say:

A deep breath, as we take in the scene. We require air to survive. As a consequence, we can rarely see beyond it. We are sixty percent water, and because air is less dense than us, we associate it with a void. This allows us to view the atmosphere as something we cannot affect. Such is the epistemological narcosis that has led us all the way to climate change. Today, we must pass through this state of intoxication into controlled dreaming - a new environmental reverie. One where our sense of individuality - a cultural complex - diffuses, slightly. Here, below the surface, in the water, above the coral, we feel more within the environment; living ourselves as bigger, overflowing terrestrial selfhood. We feel set in motion by things we only partly comprehend; physically compressed, made smaller. Here, this minute, we can dominate neither animal nor current. And we cannot stay forever. We are an impermanent phenomenon. And yet, we read our bodies in the space surrounding. Above the water, if you swing your arm, it is rare to see a reaction in a nearby tree. Your impact can be imperceptible. In diving you learn, in a visceral way, what particle physics has proved in the abstract: to observe is to influence. One kick, and a bank of nearby algae begin to flutter. (emphasis in original)

${ }_{18}$ There is much debate over the extent to which Glissant's (1997) Poetics of Relation was influenced by Deleuze and Guattari. For some, the connection is more straightforward (Hallward, 2001) than others (Burns, 2012; Allar, 2019). But what is not questioned is their understanding of the importance of thinking with islands. As Lorna Burns (2012: 1) points out, thinking with islands was 'at the heart of Deleuze's philosophy'; where islands were understood as emblematic sites of 're-creation, not the beginning but a re-beginning that takes place.' (Deleuze, quoted in Burns, 2012: 2). As in the case of Derrida (2011), Deleuze understood the central importance of thinking with islands as a way of challenging modern or mainland reasoning. As noted, here we find Drabinski (2019: 100) particularly useful when it comes to grounding Glissant's approach in island geographical specificities 
and materialities; when he says that 'Glissant's ontology of the subject emerges out of [thinking with Caribbean islands and islanders], rather than intervenes upon, space, time, language and history. In fact, siting that emergence [in the Carribean] is essential for moving from generalized Deleuzian geophilosophy to the specific geography of Glissant's subject.' 


\section{Correlation: Registers of Change}

\section{Introduction}

In the second half of this book we turn to examine how, after the end of the world of the modern imaginary, questions of knowledge and epistemology are not entirely separate from those of ontology but are onto-epistemological: i.e. knowing is not merely a product of the mind engaging in passive reflection of a world as object 'out there', but rather an ongoing process of embodied engagement and interaction. Here we suggest that dominant approaches to knowledge in Anthropocene thinking can be heuristically grasped by highlighting two points on an onto-epistemological continuum: Correlation (Chapter 4) and Storiation (Chapter 5). Central to both, in our analysis, is how islands are worked with as notable sites of relational entanglements in order to generate new approaches to knowledge and understanding. Both approaches to knowledge depart from key assumptions of the modern epistemic imaginary and are posthuman or more-than-human in orientation.

Where distinctions can be heuristically drawn between them is in how they approach, register or 'read' the Anthropocene. Correlation is a relational onto-epistemology which relies heavily on patterns of repetition and stable relations of surface effect. Here

How to cite this book chapter:

Pugh, J. and Chandler, D. 2021. Anthropocene Islands: Entangled Worlds.

Pp. 109-140. London: Ubiquity Press. DOI: https://doi.org/10.16997

/book52.d. License: CC-BY 4.0 
island life and island cultures emerge as important figures for developing Correlational approaches which sense and register the Anthropocene; illustrated well in how the island has become the emblematic 'canary in the coalmine' for climate change (Cantieri, 2017; Fitzpatrick and Erlandson, 2018; Chandler and Pugh, 2020b; West, n.d.; Baldacchino, 2020). By contrast, Storiation offers a more speculative, disruptive and generative set of openings; problematising the modernist assumptions of time and space which remain in place in Correlational approaches. This is illustrated in work which draws widely upon island life and cultures to foreground that the traces, hauntings and legacies of modernity and colonialism are not past but very much constitutive of the present (Alaimo, 2016; Morton, 2016a; Sharpe, 2016; King, 2019; Barad, 2019; Neimanis, 2019; Farrier, 2019; 2020; DeLoughrey and Flores, 2020).

In the first half of the book, relational ontologies were grasped by way of the analytics of Resilience or Patchworks. We posited a spectrum or continuum moving from the fixed, more closely bounded island relational interdependencies of Resilience, to the more open, flowing and contingent island knots of interconnection of Patchworks. In the second half of the book, where we consider the sliding scale or continuum of approaches that work with islands onto-epistemologically, we draw a parallel process of movement away from modernist grounds. The direction of travel from Correlation to Storiation increasingly takes us away from the ability to have knowledge of a law-bound universal 'nature' as assumed by modernity's human/nature divide, and seeks to put the materiality of islands and embodied intra- (rather than graspable inter-) relations to the fore as enabling thought rather than merely being an object of it. Thus, we develop the central theme of our book: how work with islands after the end of the 'world' is enabling and enriching Anthropocene thinking.

Key to the onto-epistemology of Correlation, discussed in this chapter, is the capacity to see, sense or register processes of becoming beyond those 'given' in appearance to a human subject. Correlational approaches depend on contextual relations and 
regularities, where experiential knowledge enables signs and signals to be read as indexing or registering other or unseen changes. For example, a dog barking in the night-time might alert someone to an intruder: the bark indexes something that would otherwise be unseen. Correlational forms of knowing or sensing thus enable a wide spectrum of interactions in the human and the nonhuman world and, as we later explore, are understood to inform the interactive evolutionary processes of life itself, as life forms co-relate in ecosystem processes of mutual adaptation. Correlation is not specific to human knowledge systems and, in modernity, was long sidelined in favour of the truths generated by the laws of causation. After the end of the world as imagined in modernist ways, Correlational approaches have increasingly garnered the attention of policymakers and academics and, for this reason, have often been drawn from island practices and imaginaries where these forms of working are understood to be more central to everyday life (Intergovernmental Panel on Climate Change, 2007; Benwell, 2011; Hanna and McIver, 2014; Walshe and Stancioff, 2018). In particular, the ability to read the signs of changing island environments via the practices of Indigenous island cultures is widely understood as key to registering the forces of the Anthropocene (Salick and Ross, 2009; Camus, 2018; Suliman et al, 2019; Forest Peoples Programme, 2019).

Correlational analytics focus upon how entities or 'actants' have particular capacities or affordances which can be instrumentalised to enable human knowledge of changing environmental conditions. Correlational approaches thereby often rely upon the properties of correlational techniques and assemblages to measure or register effects (such as the widely held sensitive affordances of island ecological systems or cultures to register changing environments). Entities do not therefore have a core essence or meaning in themselves, as they do in modernist reductionist frameworks of reasoning; rather, knowledge is established co-relationally. Nevertheless, Correlation is still reliant upon an object of knowledge with reproducible and predictable properties and a knowing human subject who is capable of 'standing outside', doing the 
correlating, reading and measuring of inter-relations. Knowledge is about building up increasing correlational efficiency over time, assuming a set of regularities of relation, which can be grasped. In Correlational analytics, registrations of one entity through changes in another - intensities of heat through the expansion of mercury in a thermometer, levels of carbon monoxide in the air through changes in the body of a canary, or the evolution pathways of island species - reveal changes in intensities and distributions of entities that cannot be perceived directly and thus add to human capacities to know and act instrumentally in the world. Correlational analytics, widely developed by working with islands and island cultures, can thus be 'exported' as a set of instrumentalising techniques or useful practices, which are replicable. The rest of the world can learn from island practices and the ecological sensitivities of islands life and cultures.

The chapter unfolds in three sections. The first expands upon the underlying logics of Correlational onto-epistemologies. It examines how working with islands - and the correlational tropes of the island as materially indexing or registering climate change - is central to the wider generation of Correlational approaches in Anthropocene thinking. The second section focuses upon how Indigenous islander practices, and the proliferation of digital sensing technologies and the 'smart island' concept, are playing a productive role for the development of Correlational approaches in much contemporary thinking. These work with the long-held notion that islanders and island life are particularly sensitive and are attuned to register and read environmental change in ways that the rest of the world can learn from. The third section develops the analysis further by engaging the shift in contemporary scholarship, taking Correlational analytics beyond the knowing human subject, or the knowing islander, able to register inter-relations, towards the Correlational practices of (island) life itself in the Anthropocene. In conclusion, we draw out how other trajectories associated with working with islands in the Anthropocene start to blur the dividing line between the onto-epistemologies of Correlation and those of Storiation - which we go on to discuss in the next chapter. 


\section{The Analytics of Correlation}

As Elizabeth DeLoughrey (2019) notes, islands have become vital interpretants in the Anthropocene for mapping and modelling indirectly, through the registration of effects, the impact of complex transformations in planetary conditions (Hayward, 2018; $\mathrm{Wu}$ et al, 2019). This is illustrated in an extremely wide range of island practices today: from the extensive use of Big Data to map changing island coastlines and rising sea levels (United Nations Climate Change, 2019), to the remote sensing of coral bleaching as a bio-sensor of environmental change (Li et al, 2011; Mohanty et al, 2013; Foo and Asner, 2019), the growing interest in applying algorithmic correlation to social media feeds to register emerging island disasters (Whyte, 2017), and Indigenous island peoples' own capacities for sensing climate change (Percival, 2008; De Souza et al, 2015). The recent success of such books as Robert Macfarlane's (2019) Underland, Gleb Raygorodetsky's (2017) Archipelago of Hope, and Laura Watts' (2018) Energy at the End of the World: An Orkney Islands Saga, reflect how islands and islanders are widely seen as key detectors or sensors of climactic variations in the Anthropocene; understood as important models for future sustainability and as corollaries for the survival of the human species generally' (Fitzpatrick and Erlandson, 2018: 283).

Islands are often seen as 'canaries in the coalmine' in debates about the Anthropocene because they are widely understood as small and extremely vulnerable to catastrophic climate change, and such forces as atmospheric pollution, rising sea levels and plastic pollutants (Cass, 2018; Grydehøj and Kelman, 2017; Keim, 2019). Thus, there is something to working with island affordances and properties that matters for these debates. Here, we do not think it is helpful to understand islands as 'blank spaces' for these developments, devoid of meaning, simply awaiting the 'parachuting in' and 'testing out' of Correlational onto-epistemologies. Rather, we argue that working with islands as sites of relational entanglements, affordances and feedback effects has been important for the particular development pathways and generation of correlational analytics in Anthropocene thinking more widely. Indeed, 
the figure of the island has emerged as a central way of registering, sensing or revealing processes of anthropogenic influence which would often otherwise go unseen. The emergence of islands is clear when we think back to UN Secretary General Kofi Annan's comments to the UN General Assembly in 1999, that 'islands are microcosms for our world. We are all inhabitants of the global island, surrounded by the limitless ocean of space. If we can find solutions to the special vulnerabilities of islands, it will help us address more global problems.' This is a far cry from today's framing of islands, not merely as passive victims, but increasingly as active and productive agents - 'inspiring champions' (De Souza et al, 2015: 1) which the rest of the world can and should now learn from (Hall and Sanders, 2015; Intergovernmental Panel on Climate Change, 2007; Nwanze and Sinon, 2013).

As Grydehøj and Kelman (2017: 107) have noted, 'just as the boundedness of small islands makes their beauty more graspable, it also sets their disasters in relief, transforming islands into symbolic carriers for mainland fears.' ${ }^{1}$ We take this observation as a starting point for analysing the work of the analogy of the island as the canary in the coalmine, and for drawing out its central and powerful organising logic. Our key argument is that the influential analogy of the island as the canary in the coalmine points to how islands are increasingly reinterpellated not merely as a 'living laboratory' (Watts, 2018: 105), in the sense of being small and confined sites for investigative research for the rest of the world, as they are often portrayed in the literature (Edmond and Smith, 2003; Grydehøj and Kelman, 2017; Watts, 2018; Baldacchino, 2020). More fundamental than this, we suggest, is that working with islands enables the generation and proliferation of correlational epistemologies as an alternative, moving beyond the modernist episteme's focus upon causal relations.

The registration of effects - the capacity to see processes of becoming beyond those 'given' in appearance to a human subject - is a product of the specific affordances of the particular subjectobjects, or 'actants', in the terminology of Actor Network Theory (Latour, 2005), enrolled in the process. As discussed above, 
correlational epistemologies are not new per se. Rather, in the Anthropocene, the limits of the modern episteme and the importance of process ontologies, and thus working with islands in particular as sites of relational entanglements, increasingly comes to the fore. Whilst in this chapter we focus upon islands and islanders as key Correlational registers in the Anthropocene, everyday examples of Correlational techniques would also include such mundane, epistemological instruments as the thermometer (registering air temperature based on the affordances of mercury or other liquids, which expand or contract at a constant rate as temperatures change) or the compass (registering magnetic fields based on the affordances of magnetic materials or 'lodestones' in relation to the magnetic north) (Chandler, 2018a). Thus, Correlational machines - thermometers, compasses, or islands in the Anthropocene - bring unseen or unrecognised forces into a wider awareness, thereby expanding our 'world' by revealing agential forces to us indirectly via their effects. Human, nonhuman and technological aids have long histories of enabling responsivity via the sensing or registration of effects, through the power of co-relation or Correlation. Today these approaches have become increasingly central in the quest to reveal dangerous underlying changes in planetary conditions. ${ }^{2}$

Here, working with islands is a productive force. We noted in the last chapter that, as Donna Haraway (2016: 56) points out, it was the ecologies, affordances and properties of islands and their surrounding oceans in particular which brought the Anthropocene into the consciousness of the wider world 'in the first place':

From the start, uses of the term Anthropocene emphasized humaninduced warming and acidification of the oceans from fossil-fuelgenerated $\mathrm{CO}^{2}$ emissions. Warming and acidification are known stressors that sicken and bleach coral reefs, killing the photosynthesizing zooanthellae and so ultimately their cnidarian symbionts and all of the other critters belonging to myriad taxa whose worlding depends on intact reef systems. Corals of the seas and lichens of the land also bring us into consciousness of the Capitalocene, in which deep-sea mining and drilling in oceans and 
fracking and pipeline construction across delicate lichen-covered northern landscapes are fundamental to accelerating nationalist, transnationalist, and corporate unworlding. (Haraway, 2016: 56, emphasis in original)

It is important here to illustrate how the trope of the island and its surrounding environments, as notable registers for climate change, shifts the focus to sensing and Correlation, rather than a modernist ontology of causation, as this is significant to the importance of islands as instruments for non-modern ways of working in the Anthropocene. Correlation relies on causal laws or regularities, but the key aspect is that these are secondary to Correlation rather than primary. As Bruno Latour argues, Correlational epistemologies are not about entities or essences but relations: the causal becomes background to the relational effects which are foregrounded (Latour et al, 2011: 84). In the classic trope of the canary in the mine, the precondition for the canary signalling the existence of carbon monoxide is the causal regularity of poisonous gas killing the canary before mine workers are aware of its existence and prone to its effects. However, the problem of carbon monoxide is not addressed at the level of causation (predicting it or preventing it from appearing or solving the problem afterwards) but through developing a method of signalling the existence of poisonous fumes and of increasing human sense-ability through the power of Correlation. Without this registration of effects, carbon monoxide is understood to either exist or to not exist in a mineshaft, and by the time it exists it is too late and the coalminers die.

The addition of the canary into the situational context reveals the coming into existence of other actants, the poisonous gases, which would have previously operated unseen, beneath the level of human cognition. The affordances of the canary enable poisonous gases (variations in intensities) to become quantified or measured via the material body of the canary. In the same way, the fact that mercury expands when heated is a specific capacity or affordance that enables enrolment in a technical more-thanhuman assemblage - a thermometer - or Correlation mechanism. 
As Scott Schwartz (2017) writes, these affordances enable the translation of an intensity, like heat, to be read or made legible through extension, in the form of measurement; thus, enabling something that cannot be seen directly to be datafied indirectly. In short, Correlation can translate quality into quantity, enabling its registration through effect. Intensities such as air temperature or gas densities thereby come into existence as meaningful or legible objects.

Anthropocene thinking is fundamentally marked by new approaches which seek to affirm the enabling powers of morethan-human relations. For such authors, the power of the Anthropocene (Danowski and Viveiros de Castro, 2016), 'Gaia' (Latour, 2017; Stengers, 2015), the lithosphere (Clark and Yusoff, 2017), or 'hyperobjects' (Morton, 2013), like global warming, while too great for the human intellect to grasp in modernist forms of 'command-and-control', enable new forms of thinking and responsivity to emerge. Although 'anthropos' may have forged the road to the Anthropocene, the tables are turned; our transforming planet is setting the pace, revealing to us the overwhelming power and forces of more-than-human relations. Humans are now tasked with following and responding to these forces, having a more humble role: to learn how to better Correlate and sense what the transforming planet is telling us (Chandler, 2018b; Chandler and Reid, 2018; Chandler and Pugh, 2020b). The problematic becomes that of: 'how to listen?' and 'how to become aware?' The sciences of correlation rather than causation and the need to develop new methods and approaches of onto-epistemology - correlational technologies - have thereby come to the fore.

The ecological sensitivities of island life in particular mean that, for many commentators on the Anthropocene, it is islands which are sounding 'the alarm for climate change' (Cass, 2018). The breadth of research which understands island life as a Correlational mechanism is significant; thereby enrolling such varied island 'actants' as penguins (Carravieri et al, 2013), moose skulls (Berman, 2017), insects (Jongejans, 2019) and beach width (Mann and Westphal, 2014) as sensors of global warming. The particular sensitivities and affordances of island life and island 
cultures are said to make them extremely important for engaging the overarching problematic of Anthropocene thinking: reworking relational entanglements as enablers of rather than barriers to knowledge, via new capacities for sensing changing and transformative conditions.

\section{Islanders' Correlational Practices}

As discussed in the previous chapters, the question of how Indigenous islanders' sense and register the world around them differently from Moderns is at the heart of many wider debates about the Anthropocene (Forest Peoples Programme, 2019; Ellsmoor, 2019). Anthropologists and other researchers have done much to foreground how islanders' forms of spatial and temporal awareness are key to unlocking more productive ways of registering the Anthropocene (Percival, 2008; Salick and Ross, 2009). In particular, there is a strong critique of 'Western preoccupations with separating ontology from epistemology, knowing from being ... [in favour of an] Indigenous conception of onto-epistemology' (Kanngieser and Todd, 2020: 385; Watts, 2013). For Kanngieser:

From what I have been told of Pacific cultures, it is impossible to separate land from oceans, people, plants, animals and spiritual worlds. Konai Helu Thaman, a poet and scholar from Nuku'alofa, Tonga, states that 'Pacific notions of identity tend to emphasise the 'environment' in its totality, a concept for which the English term 'land' is grossly inadequate.' Unaisi Nabobo-Baba explains that in Indigenous Fijian languages the word vanua denotes 'land as well as place ... everything on it and in it ... all flora and fauna as well as waterways, oceans, mountains and forests ... Land is of physical, social and spiritual significance to people.' Within Pacific conceptions of environment, writes Banaban, I-Kiribati, and African American anthropologist Katerina Teaiwa, the ocean is a 'corporeal and psychic relational vehicle,' and land serves to teach 'about the 'spatiality' of life in contrast to or in concert with the sea.' When non-Pacific and non-Indigenous scholars generalize any relation to land, they erase these formative knowledges. 
Universal discourses in Western environmental histories are inadequate if they do not recognize that place and land are shaped by relationships that are not interchangeable. When Land is understood in this way, kin studies might proceed. (Kanngieser and Todd, 2020: 388)

Not only thinking about but with these island cultures is today seen as productive and generative. ${ }^{3}$ As modernist frameworks of reasoning and scientific knowledge seem to reach their limits in debates about the Anthropocene, island life, and Indigenous islander ways of knowing, through the Correlational registration or sensing of effects, appear to provide a non-modern alternative (Māhina, 2008; De Souza et al, 2015; Vaai and Casimira, 2017; Fair, 2018; Falefou, 2017; Farbotko, 2018a, 2018b). Engaging Indigenous islanders' correlational practices and worldviews are said to be particularly crucial because:

Indigenous science and knowledge are based largely on bioindicators, or natural signs ... Many animals can sense earthquakes and other natural disasters before humans can, and watching their behavior can give us time to get to safety if such an event occurs. Learning from nature in this way is an integral part of the Indigenous worldview that all things are connected, and that nature, when respected, can be a benevolent part of the whole community. (First Peoples Worldwide, n.d.)

Working with island cultures' correlational worldviews is widely understood as an important antidote to the hubris of modern reasoning - a better way of reorienting to the higher stakes in the Anthropocene. ${ }^{4}$ Thus, Suliman et al (2019: 300) highlight that:

The ancient Austronesian concept of *banua [meaning 'land', 'home' or 'village'] suggests an unfolding, emergent and yet holistic system across space and time; a complex network of mobilities and immobilities connecting people, ancestors, stars, canoes and other vessels, ocean, islands and continents. This system, perhaps best conceptualised as a dynamic cosmological compass, originated in South-East Asia [probably in Taiwan] with the ancestors 
of Pacific Islands settlers. ${ }^{\star}$ Banua... forms a cultural scaffold for past, present and future (im)mobilities in and around the Pacific Islands, and provides an orientation for thinking about Anthropocene (im)mobilities within and beyond the region.

Whilst a large amount of island scholarship has examined the sophisticated navigational practices of islanders across vast oceans - especially islanders from 'Oceania' (Lewis, 1994; Finney, 2003; DeLoughrey, 2007; Hau'ofa, 2008; Genz, 2011; Rakuita, 2017; Perez, 2020a) - here Suliman et al's (2019: 300) framing of *banua as a 'dynamic cosmological compass' seeks to inform Anthropocene thinking by articulating an Indigenous islander cosmological compass and Correlational worldview. ${ }^{5}$ Indeed, as these researchers point out, navigation across oceans is in fact secondary, or subsumed, to *banua's wider cosmological compass: 'an active culturally and physically nourishing *banua in the Pacific Islands seems to endure before, during and beyond the spatial and temporal passages of those who call it home' (Suliman et al, 2019: 311). ${ }^{\star}$ Banua is an extremely sophisticated Correlational worldview which facilitates the 'constant repositioning of the self with reference to the moving cosmos' (Suliman et al, 2019: 312). As Suliman et al (2019: 311) quote Māhina (2008: 76) with reference to the Tongan, local variation of fonua:

On the universal level, fonua entails the dialectically changing formal, substantial and functional relationships within and between nature, mind and society ... On the unique level, however, fonua espouses the historically shifting ecological, psychological and sociological connections within and across fonua (birth), fonua (living) and fonua (death), as conflicting physical, emotional and human processes of eternal cycle and exchange.

For Suliman et al (2019), working with islander Correlational compasses and worldviews is not just an interesting exercise in Anthropology, it draws wider attention to important forms of practical mobile ways of knowing which exist outside formal governance spaces and processes. Highlighting this therefore 
becomes a way of 'challenging state-centric approaches to climate change adaptation' and is seen as 'essential for the existential security of Pacific people and central to contemporary climate activism' (Suliman et al, 2019: 298):

Expansive, open and shared across the multiple indigeneities of the Pacific Islands since original settlement, ${ }^{*}$ banua seems likely to endure beyond the Anthropocene through ongoing, changing and yet also eternal mutual custodianship of life with ancestors and descendants. The mobile nature of ${ }^{*}$ banua is lived in Pacific Island diasporas in places like New Zealand and Australia, and is likely to survive even the worst case scenario of complete loss of habitability of some islands. ${ }^{\star}$ Banua is likely to continue to offer cosmological resilience in a changing climate, even in the face of individual, family, community or national despair arising from loss of land in the *banua, possibly in new, as yet unknown ways and perhaps most importantly, whether remaining on or leaving degraded lands amid rising seas, its people can continue to nourish *banua and be guided by it. A partial balm, perhaps, to the experience of profound existential insecurity. (Suliman et al, 2019: 313)

Such passages draw attention to how working with islanders' correlational practices and worldviews is frequently seen to provide alternative, more productive, ways of generating knowledge. As another example, Camus (2018: 146) argues that greater anthropological insight into how islanders from Kiribati register, sense and correlate to their environment has an important 'role to play in the debate on "adaptation" and "resilience", for it can humbly act as a kind of agency or sentinel alerting us to the reality that this debate cannot stay in a state of suspension much longer.'

The alignment here of Resilience ontologies with Correlational approaches to knowledge is not unusual in the Anthropocene literature. Both Resilience and Correlation adopt a logic of relation, with the knowing human subject being understood as capable of reading the patterned regularity of inter-relational effects - such as the islanders who read the 'dynamic cosmological compass' of *banua (Suliman et al, 2019: 300). Both Correlation and Resilience 
also involve a much wider or 'flatter' redistribution of agency than top-down, causal, modern frameworks of reasoning which operate according to the logic of a human/nature divide. For Resilience analytics, focusing upon relation and interdependency enables greater adaptation to the forces of the Anthropocene by drawing upon the immanent potentialities of interactive (island) life itself. For Correlation, as an onto-epistemology there is a complementary focus upon working with islands and island cultures as a way of sensing, revealing and generating greater knowledge of complex relational patterns and connections. Moreover, because both Resilience and Correlation draw upon generalised patterns of knowledge, forms of reading or sensing, they can be instrumentalised, exported, and made replicable as wider practices for engaging the Anthropocene - for example, as noted, *banua has endured and travelled over many generations, and there are multiple localised variations spanning Austronesia, which, Suliman et al (2019) argue, enhances islander Resilience in the Anthropocene.

The extension of the logics of Correlation is not confined to interest in Indigenous islander correlational practices. Correlational logics are also driving the development of new sensing technologies which work on the assumption that islands and island cultures are particularly sensitive to changing environmental conditions. As Jussi Parikka (2015) has highlighted, as far back as Lyell, Darwin and Babbage, in the 1800s, the Earth has been pictured as a giant sensing mechanism, with Babbage (1837) arguing that '[t]he air itself is one vast library' (see also Parikka, 2015: 138). In 1839, John Ruskin pictured a 'vast machine ... systems of methodical and simultaneous observations ... omnipresent over the globe, so that [meteorology] may be able to know, at any given instant, the state of the atmosphere on every point of its surface' (quoted in Edwards, 2013: 431). Today, there is a massive interest in the development of Correlational machines capable of sensing the changing conditions associated with the Anthropocene; where 'synthetic computation expands what is sensed, measured, calculated, communicated, stored and worked on' (Bratton, 2015: 87-88; Springer et al, 2017). Reflecting the prominence of islands as Correlational 
machines in these developments, Springer et al (2017: 18) powerfully argue for the 'relevance' of these approaches 'for a contemporary consideration of the concept of the island as such'.

The characteristics of islands as enclosed relational spaces of interdependency are often said to make them 'by their very nature, agile in size and governance - useful factors to become an innovation "testbed" (Handforth, 2017; see also Grydehøj and Kelman, 2016, Baldacchino, 2020). 'They can move quickly to trial and scale new technology, providing innovators - big and small - with realworld environments for testing new ways of working' (Handforth, 2017) - from the call for new 'smart islands' which could sense emergent effects and enable 'real-time decisions' in the Caribbean after Hurricane Irma (Whyte, 2017), to the real-time detection of changes in air and water quality on islands (Smart Island World Congress, 2019), to tracking the fluctuating levels of food available in retail shops after island disasters (Cavallo, 2017). Island Innovation's The Virtual Island Summit (September, 2020), led by James Ellsmoor, is just one illustration of the popularity of these approaches, attracting around 10,000 attendees. Here, as just noted, Correlational approaches often work productively with the analytic of Resilience, and the types of examples discussed in Chapter 2, expressed in the notion of smart 'Islands of the Future' (Filmproduktion and Arte G.E.I.E., 2016). Again, in these developments, it is extremely important to note that islands are not simply 'blank spaces' or 'empty laboratories' devoid of meaning. It matters that such digital sensing technologies are generated by working with islands as widely understood sites of adaptive potential, relational sensitivities and feedback effects. Thus, these existing affordances are readily available for the construction of new digital approaches seeking to make these relational effects legible to planners and policymakers.

'How do you turn these islands into a living IoT [Internet of Things] lab? Just add 500,000 sensors' (Solana, 2017). In Spain's Balearic Islands, referred to in this quote, the movements and relational interactions of island life can become seen or are 'datafied' through their translation into digital sequences, via their 
registration through sensory equipment, now so cheap as to become increasingly ubiquitous. Perhaps the most obvious example of this is Singapore, where, as Smart Island (no date) journal says:

Making technology all pervasive, permeating every sphere of activity, Singapore became an Intelligent Island by year 2000 ... But technology does not cease to evolve, so Singapore has a constant focus on it and now has a 10-year plan to become the world's first Smart Nation by 2025! Sensors will be rolled out across the country to further improve the quality of life for its citizens. ${ }^{6}$

Such digital sensing operates through Correlational logic enabling the unseen to be seen through the registration of effects, in these cases, upon the material body of the sensor. On the island archipelago of Indonesia, the capital city Jakarta has sought to turn its citizenry into citizen-sensors, capable of early detection and adaptive responsiveness to wide-scale flooding. One such Correlation and sensing project, PetaJakarta, sees the population of the major city as a resource still in need of mobilisation: they are already extensively networked through social media and could make great citizen-sensors, especially once flood information offered can be verified through geo-spatial tagging of the precise time and location (this enables others to check and compare the information from multiple sources and makes verification much easier) (Chandler, 2017). Social media can be reconfigured with humanitarian apps to activate these civic citizenship elements. Different problems can then be used to construct engaged and active communities able to play a role in addressing them as a form of 'civic co-management' (Interview, PetaJakarta Coordinator, 2016, in Chandler, 2017: 118). The development of civic communication technologies is understood as enabling a more dynamic reality of island life to unfold, amplifying the collective networked social intelligence of the island city, where the citizens and the river flooding work together to reveal the river's importance and to develop syncopated rhythms of adaptation, rather than seeking to control or 'normalise' the river system (Chandler, 
2017). At present, new civic technologies are being bankrolled and tested in relation to disasters and emergencies, but the hope is that this could be the beginning of new forms of geo-social networked systems enabling much more distributed and democratic forms of real time island governance.

Another localised illustration of how Correlational analytics are associated with the development of new sensing technologies can be found in Elizabeth Johnson's (2017) insightful examination of the work of commercial bio-sensing and the use of organic life to monitor fresh and marine water sources for pollution. Here an array of animal species, including small fish, worms, molluscs, crustaceans and micro-organisms are monitored intensively to discover their norms of functionality and to develop ways of measuring changes in these indicators. They are then ready for use as correlational technologies of registration:

[The company] monitors a suite of 'behavioral fingerprints' as these organisms are exposed to different systems. Locomotor activity, reproductive rates, and embryonic developments are measured together to indicate the severity of hazardous anthropogenic chemicals as well as biologically produced toxins, such as blue-green algae. In this way the company boasts, it can make 'pollution measurable'. (Johnson, 2017: 284)

As Johnson (2017: 284) notes, this mode of generating knowledge is less about causation than seeing indirectly via effects: making 'imperceptible harms' perceptible. The approach sees through Correlation, which enables new problems and possibilities to be detected. Changes in the bodily indicators of the animal organs can alert human agents to potential problems, even if the sources of those problems are unknown. Thus, the company concerned argues that problems can be detected in due time before pollution irreversibly spreads in the environment or even harms human health' (Johnson, 2017: 284). In a technological extension of the nonhuman prosthesis of the canary down a coalmine, bio-sensing becomes a powerful way of 'sensing the Anthropocene' (Johnson, 2017: 275). Intensities of pollutants or toxins are given extension or 
appearance through the affectivities of the bodies of small marine creatures. This form of knowledge generation works on the basis of developing new forms of correlational sight; enabling a fundamental shift from knowing on the basis of analysis of causal connection to the adaptive knowledge of registering surface effects. The onto-epistemology of Correlation is not concerned explicitly with the direct essence of entities, or with chains of causation, but with seeing emergent effects; enabling 'more-than-human' assemblages of responsivity. New actors or agencies are brought into being through the affordances of islands' ecological sensitivities, enabling the appearance of 'effects', and thus enabling insight into processes of emergence through these 'co-relations'.

The underlying logics of Correlational approaches, and how islands are regularly enrolled in their development, are also usefully highlighted in Stephanie Wakefield and Bruce Braun's (2019) work on the deployment of 'green infrastructure' on Manhattan island. This also relies on the agency of nonhuman actors, such as the deployment of oysters as seawall infrastructure, to enable sensing that is grounded in responsivity. Wakefield and Braun highlight the distinctiveness of this mode of governance which, rather than seeking to adapt and learn on the basis of causal relations that are oriented towards the future, has a very different temporality or approach to the future in that it seeks to 'ward it off' (Wakefield and Braun, 2019: 13: emphasis in original); attempting to keep everything as it is by cancelling out or absorbing events. Rather than seeking to reform or adapt existing modes of infrastructure - for example, by building walls around Manhattan island - such approaches instead seek to maintain existing forms of infrastructure but to add other forms of sensing and responsivity. While modernist or causal understandings assumed a hierarchy of centralised reporting and adaptation, such Correlational governance has a much flatter ontology of self-generated responses, whether at the level of society, community or the quantified self.

Such innovators regularly work with island life in order to develop and forward Correlational onto-epistemologies as an important way of engaging with the environmental changes of 
the Anthropocene. Some of these do so by developing approaches which examine how island life itself is quite literally sounding the alarm bell of changing planetary conditions. As Lewis Gordon (2018) examines in an article 'What does the End of the World Sound Like?', an increasing number of researchers are recording the changing soundscapes of islands undergoing rapid environmental change; for example, changing bird songs, dogs barking, the sounds of forests, cyclones and islanders (for a good example see the 'Burrow Collective' (2020) on Fiji). For Anja Kanngieser (2020), islands are key sites which enable us to listen to the sound of ecocide as it unfolds. It is the affordances and sensitivities of islanders and island life in particular which repositions working with islands as central in these contemporary debates, not in modernist ways - needing to be protected and saved - but as spaces in which new approaches to sensing relational entanglements can and should be developed. Just as the Anthropocene at one and the same time puts humans at the centre of the problems of climate change but also weakens and undermines claims to human superiority, so islands and islanders are seen as undermined and threatened in the Anthropocene; but, importantly, also become key to sensing changing climactic and environmental conditions.

\section{The Correlational Practices of Island Life}

Whilst many academics and practitioners give attention to islander correlational worldviews and practices, Anthropocene work has also focused upon island dynamics themselves as a correlational practice. In order to demonstrate the wide-reaching power of thinking with islands, here we expand the ways in which island dynamics are being engaged by turning to ways in which ecosystems, such as forests, can be thought in terms of island dynamics (Burgess and Sharpe, 1981; Howe, 1984; Small and Hunter, 1988; Rolstad, 1991; Bierregaard Jr et al, 1992; Iida and Nakashizuka, 1995; Edwards et al, 1999). A good example of this is Eduardo Kohn's How Forests Think (2013). For Kohn, the Amazonian rainforest enables us to work with island characteristics of an isolated 
system of relational interaction and feedback; and thus the generative power of embodied forms of knowledge as the logic of life itself - as Correlational - is clearly illustrated in Kohn's approach of a material semiotics of interactive life as becoming. As in the case of Darwin's work on islands (2010), island relations are seen to work immanently to magnify or intensify island differences and distinctions. Thus, island forms of embodied knowledge multiply or pluralise the world rather than reducing or homogenising it. Central, for Kohn (2013), is how this process of island becoming is correlative; where patterns repeat and flow through life as material signs and registrations are read and responded to. To give a simple example, the presence and distribution of water will structure the distribution of species of plants and insects, which will shape the distribution and nature of animal species and so on. Life thus 'correlates itself' interactively to ever higher levels of complexity. As species, including humans, seek to harness life's powers and resources, these patterns become magnified. Correlation, in this sense, is a materialised set of interpretations and reinterpretations. The world becomes readable or registerable in its materiality through its relationships of feedback and their regularities and patterns.

Working with island forms of embodied knowing and interrelation (here, the rainforest as a distinct 'island' ecosystem) enables understandings of life that go beyond linear or deterministic imaginaries; in fact, emphasising non-linear and multiple potential developmental paths. For Kohn (2013), for example, the giant anteater is a contingent relational product but nevertheless it expresses and amplifies the reality of the world in relation. We cite a passage to illustrate:

Anteater snouts over the generations have come to represent with increasing accuracy something about the geometry of ant colonies because those lineages of 'protoanteaters' whose snouts and tongues less accurately captured relevant environmental features ... did not survive as well ... today's living anteaters have come to exhibit comparatively increasing 'fitness' to these environmental features. They are more nuanced and exhaustive representations 
of it. It is in this sense that the logic of evolutionary adaption is a semiotic one. (Kohn, 2013: 74)

It is relational interdependency - in this case, the fact that the giant anteater is dependent upon ants as the sole food supply, and the regularity of its repetition over time - that enables this kind of coeval adaptation of species-in-environment. Thus, the island form of relational dependencies and interaction is that of a process which is itself a material narration. Whilst, as noted, a significant amount of work has examined forests in terms of island dynamics, as Robin (2014) says, there is 'something profound about islands in general ... They are places revealing Earth's history: the very soils and climates accelerate and concentrate evolutionary processes'. The path dependencies and interactive stories of life - prevalent everywhere but revealed prominently by island life - are conspicuously registered in the material bodies of the actants themselves. The point is not so much one which concerns the ontology of complex adaptive systems, but that of (island) life itself as a communicative process which depends upon capacities for being and becoming sensed, read, registered and Correlated - as species change and transform through interaction over time.

Kohn's (2013) semiotic approach is not forwarding an abstract claim - that every atom or grain of sand contains the history of the universe - but a highly concrete one. Life is irreducible and every life 'pathway' contains the individuated story of itself as an interactive becoming. This is readable or knowable through the traces which continue to exist and to relate relations. It is registered in these relations as life 'Correlates itself' in regular patterns and through their amplification. Importantly then, for this ontoepistemological approach, humans are not the only readers or interpreters of signs or stories. All life is held together through the Correlation of feedback effects which enable continuities to exist in ways which exceed modernist or anthropocentric understandings which separate Thought and Being, or Nature and Culture, reserving communicative interaction and cultural distinction for the human realm. The material semiotics of Kohn's, which the work shares with some advocates of Actor Network approaches (see, 
for example, Law 2007), puts material interaction at the centre of understanding rather than fixed essences of entities, which are separate or distinct from the environment. Differences continue to make differences, but for Kohn, as for many theorists considered in the Resilience chapter, there is still a telos - an underlying reality to the world which is accentuated by interactive (island) life: it is only because the world has some semblance of regularity that it can be represented' (Kohn, 2013: 59).

Working with such interactive feedback processes of islands as Correlational archives emphasises continuities or patterned regularities which enable habits of interactive adaptation to evolve. The point, as it was for Darwin, is that islands amplify or concentrate such processes, making them particularly prominent or apparent for the development of Anthropocene thinking. Today it is widely argued that working with island life enables us to develop better ways of sensing, Correlating and reading the regularities of forces associated with the Anthropocene. Eben Kirksey's (2019: 23) work, as another example, has explored the new chemosocial communities of the Australian green and golden bell frog, which have emerged in a complex landscape shaped by chemical weapons industries, municipal landfills, government remediation programs, real estate speculation, and a multitude of chemical and biological agents.' Focusing upon the legacies of dumping grounds in the Sydney Olympic Park, Kirksey examines how these bounded, but intensively inter-relational, urban islands have become a habitat for these endangered species; noting that whilst many other amphibians have been harmed by toxic chemicals, bell frogs have 'persisted here in polluted areas while vanishing from many protected conservation zones' (Kirksey, 2019: 23). Thus, 'While the normal world order of this frog has been lost with the spread of a deadly fungal disease, toxic chemicals have enabled the continuation of its social life' (Kirksey, 2019: 23).

Life thus appears to have its own immanent drive or dynamic producing a hierarchical or biopolitical ordering in which traces of the past appear as legacies in the present. This framing of interactive ordering, initiated with Charles Darwin's work on islands, further decentres Man in the sense that he becomes a creaturely 
being: 'our ancestor was an animal which breathed water, had a swim bladder, a great swimming tail, an imperfect skull, and undoubtedly was a hermaphrodite!' (cited in Alaimo, 2016: 115). Man is put back into the world of being and could be seen as composed of and in inter-species life. As Neil Shubin states, the human body itself can be read as Correlation, a material registration of effects, not just of our evolutionary history but also the history of the planet and the solar system itself:

If you know how to look, our body becomes a time capsule that, when opened, tells of critical moments in the history of our planet and of a distant past in ancient oceans, streams and forests. Changes in the ancient atmosphere are reflected in the molecules that allow our cells to cooperate to make bodies. The environment of ancient streams shaped the basic anatomy of our limbs ... The list goes on. (cited in Alaimo, 2016: 119)

However, despite the fact that, as Alaimo (2010: 158, 2016) has argued, Darwin's evolutionary insight 'gives us our first glimpse of the "posthuman", the present (usually a white Eurocentric male present) is always the apex of being and from this vantage point the past can be grasped and appropriated, even if this is conceived in nonlinear ways. ${ }^{7}$ Thus, despite his opposition to determinism, Karl Marx, for example, was to write that the ape could only be understood from the higher development of its anatomy in Man; in the same way that bourgeois society enabled a better understanding of the economies of earlier modes of production (Marx, 1973: 105). As Alaimo (2016) states, this view of the present as containing the material traces of the past can easily be subsumed under a reassuring anthropocentric story of evolutionary complexity, leading up to the present as the culmination of the process.

In debates about the Anthropocene, it is precisely the regularities and patterns of co-evolution which are under threat through catastrophic climate change, ocean acidification and island species extinction. Relational interactions are seen to work in uneven and unexpected ways. Stories of evolution on islands and perfected synergistic becoming through embodiment of the environment can often end up in tragedy, as co-dependences become a death 
sentence rather than a matter of 'the survival of the fittest.' This is captured well in the stories of 'flightways' of Thom van Dooren (2014), which we briefly discussed in Chapter 1 . Here speciation is a process not only of creative becoming, but also of extinction, where islands figure prominently in Dooren's associated projects such as The Living Archive: Extinction Stories from Oceania (The Living Archive, 2020). This includes an interactive map which seeks to track stories of species extinction and environmental degradation for the extensive range of islands it lists as the Northern Mariana islands, Guam, Marshall Islands, Federation of Micronesia, Palau, Papua New Guinea, West Papua, Nauru, Solomon Islands, Kanaky (New Caledonia), Vanuatu, Tuvalu, Kiribati, Wallis and Futuna, Tokelau, Samoa, American Samoa, Australia, Fiji, Tonga, Niue, Cook Islands, French Polynesia, Pitcairn, Rapa Nui (Easter Island), Aotearoa (New Zealand) and Hawai'i. Through such extensive projects, the Anthropocene and islands thus enable Correlational onto-epistemologies to also tell different, less positive, stories of relational interdependency. As we have seen in this chapter, this is more broadly the case for researchers who draw upon islanders' own Correlational practices and cosmological compasses to reveal how patterned relations are being disrupted in the Anthropocene, and for those who focus upon island life itself as a Correlational or sensing process. In both cases, islands have become important for generating alternative ways of knowing, sensing and revealing the disruptive forces of climate and planetary change.

\section{From Correlation to Storiation}

This chapter has explored how Correlational approaches which work with islands as key sites for understanding relational affordances and feedback effects - variously employing the tropes of islands and islanders as the 'canaries in the coalmine', Indigenous islanders' own correlational practices, new digital sensing technologies and the 'smart island' concept, and the evolutionary pathways of island life itself - can be seen to work very differently 
from modernist epistemologies oriented around understandings of causation. They instead focus upon sensing and registering the dynamic processes of inter-relations in their processual emergence. Yet, for an increasing number of commentators on the Anthropocene, these types of Correlational approaches are still too hubristic in their assumptions of Correlational regularities of relation and of a 'knowing human subject' capable of instrumentalising, assimilating and appropriating the more-than-human world in these ways.

Setting up the stakes for the next chapter on Storiation, we can turn to the growing interest in Derrida's (2011) The Beast and the Sovereign for Anthropocene thinking. Cary Wolfe, for example, has drawn upon Derrida's deconstructive contention that ' $t$ t] here is no world, there are only islands' (quoted by Wolfe, 2017: 140). The focus of Wolfe's (2017: 137) analysis is Big Bend National Park on the USA/Mexican border, 'an island of biodiversity in the vast, arid wasteland that is western Texas' whose richness of species is said to be akin to the ecological diversity found by Darwin in the Galápagos. The point which Wolfe (2017: 138) makes about the power of working with the 'conceptual apparatus of the island' is that islands are not selective because they are closed to the external world, but precisely because they are spatially and temporally open internally: 'the more systems build up their own internal complexity through recursive self-reference and closure, the more linked they are to changes in their environments to which they become more and more sensitive' (Wolfe, 2017: 149).

For Correlational approaches, these capacities and affordances of island systems are what make islands like Big Bend National Park significant sensory assemblages. But Wolfe's argument goes further and disrupts the logics of Correlation, because island ecosystems can assist expanded forms of perception: enabling us to move beyond the spatial and temporal assumptions of the modernist episteme, to grasp the existence of traces, hauntings, ghosts and plays of differences and absences. Wolfe (2017: 140) deploys Derrida (2011: 8-9): 
... [neither] animal or human individual inhabit the same world as another, however close and similar these living individuals may be (be they humans or animals), and the difference between the one world and another will remain always unbridgeable, because the community of the world is always constructed, simulated by a set of stabilizing apparatuses, more or less stable, then, and never natural, language in the broad sense, codes of traces being designed, among all living beings, to construct a unity of the world that is always deconstructable, nowhere and never given in nature. Between my world ... and any other world there is first the space and time of an infinite difference, an interruption that is incommensurable with all attempts to make a passage, a bridge, an isthmus, all attempts at communication, translation, trope, and transfer that the desire for a world ... will try to pose, impose, propose, stabilize. There is no world, there are only islands.

For Wolfe, the rich and vibrant ecology of islands can enable our awareness of this capacity for holding together multiple worlds beyond anthropocentric conceptions of flat grids of space and time. We move beyond appearances of stable entities and relations to speculatively foreground other modes of relating and interplays of affect beyond human sensibility but which make any island ecosystem what it is. Wolfe extrapolates from Derrida's provocation into a way of working with island ecosystems themselves as multiple 'worlding' processes involving different spatial and temporal interconnections, with the conclusion that:

what counts as 'world' is always a product of the contingent and selective practices deployed in the embodied enaction of a particular autopoietic living system, which is always closed and selfreferential on the level of its particular mode of 'organization' but open to its environment and its perturbations on the level of 'structure'. (Wolfe, 2017: 141, emphasis in original)

Thus, processes of interactive 'worlding', as Derrida tells us, will also always involve the influence of multiple affects beyond the stabilised appearances that constitute the 'world' of any specific form of life. These affects, speculatively grasped in terms of the 
deconstructive play of presences/absences, ghosts and hauntings, are as much a part of the present dynamics as entities which may appear to us in more stable ways. For example, changes in temperature or humidity many thousands of years ago which humans are no longer aware of, or the extinction of species which humans can no longer register or sense, or processes of colonialism, whose legacies are not readily apparent, that have enabled Big Bend to have the unique fauna, flora and animal life which it does. ${ }^{8}$ This 'haunting' should not be understood as problematic but as constructive; although often unacknowledged or unseen, these absences still hold (i.e. exist) in the present and help the present to hold (i.e. to cohere).

As Wolfe says, in practice these material tracings and hauntings, which are vitally important to making any island ecosystem what it is, will stretch infinitely in time and space, and thus it is simply impossible for the knowing human mind to be able to grasp, sense or register them in their totality. For Wolfe, this focus fundamentally challenges the presumption that there is some Archimedean point from which a human being could 'stand apart' and see that 'everything is connected' - as humanly readable, patterned regularities - on the island. Working with islands in this expansive way thus enables Wolfe to rework or extend the logics of Correlation; to expand debate into speculating upon these presence/absences, traces and hauntings which make island ecosystems such as those of Big Bend National Park. This extension or intensification of Correlational logics we conceptualise as the analytic of 'Storiation. This onto-epistemological approach profoundly disrupts the notion of a knowing human subject capable of knowing via fixed or regular patterns of interaction and affect in synchronic relations of time and space. There is, instead, a reoriented focus upon:

traces that register the presence of an absence - not just the absence of the ecosystems in which the maples, oaks, and aspens are typically found, much farther north, but a much more profound absence that challenges the commonplace notion in ecological thought that 'everything is connected,' an absence that challenges, that is, the notion of 'world' in which islands would 
be just nodes, points of interconnection in a larger, encompassing fabric of life. (Wolfe, 2017: 139)

For Wolfe (2017: 138), central to Derrida's contention that ' $[\mathrm{t}]$ here is no world, there are only islands' is that the world and islands do not exist as coherent graspable wholes, as they did under modern frameworks. Rather, the world is one of infinite islands, differences and presences/absences; where each temporal interaction carries 'a materialized 'trace', as Derrida would put it, whose inscrutability haunts [holds] the present with retentions from an evolutionary past and protentions of an evolutionary future' (Wolfe, 2017: 142143). The onto-epistemological focus of Storiation is a humbling but nevertheless enabling one, suggesting that humans can never fully grasp island reality but can generate insights when approaching islands speculatively. Working with islands in the Anthropocene by way of speculating from the materialised traces and plays of difference/ absences enables us to see islands as invitations to thinking differently and more expansively.

If Patchworks can be seen as a disruption of the modernist tendencies which still linger in Resilience ontologies, the same can be said of how Storiation disrupts the modernist, epistemological claims of Correlation to be able to read inter-relational patterns of path dependency. Here the epistemology of Storiation constitutes more fluid and contingent approaches of awareness. There is no claim to be able to coherently grasp island inter-relations, as in the case of digital Correlational technologies noted above, or the evolutionary pathways tracked by authors such as Kohn (2013) and van Dooren (2014). What is at stake in Storiation is not the ability to register or read inter-relation by employing more-thanhuman assemblages, Correlational mechanisms or cosmological compasses; but a more open, speculative onto-epistemology which registers the holding of hauntings, spectres, ghosts and legacies of such forces as colonialism, consumerism and pollution in the Anthropocene. ${ }^{9}$ It is through these forces, that can be speculatively registered in 'strange', 'weird' or 'quantum' ways, that new possibilities for nonhuman-centred thought emerges. 


\section{Conclusion}

This chapter has focused upon how work with islands and island imaginaries has been vital to informing and developing new ontoepistemological approaches, that seek to go beyond the limits of modernist frameworks of knowledge. We have seen above how Correlational onto-epistemologies bring thought into the world, mobilising the power of relation to co-relate understandings via materialised registrations, marks or signs that emerge as a material effect of relational interaction, independently of whether there is a human subject present. New correlational knowledge capacities are, for example, given a material form in digital sensing technologies and the 'smart island' concept; where the focus is upon the emergence of effects but there is no assumption that effects can be understood and manipulated or governed through the imposition of external or subject-centred policy goals. ${ }^{10}$ Real time responsive forms of management through digital sensing, switch the focus to the 'what is' (Latour, 2013: 126) of the world in its complex and plural emergence. Latour (2017) argues that such machinic or more-than-human methods of 'onto-epistemological' knowing are absolutely necessary today, because modernist forms of representation, reduction, abstraction and exclusion cannot know a world that is plural, lively and interactive.

The materiality of the relational becoming of the world, brought to the fore in island work, is a vital mechanism of decentring human- or subject-centred approaches to knowledge. Thus onto-epistemological approaches are associated with what is often called the 'ontological turn' which seeks to expand our world, not by adding one more human-centred cultural perspective but a less human-oriented way of seeing or perceiving itself (Holbraad and Pedersen, 2017). ${ }^{11}$ Onto-epistemology is about the material embodied affordances of worldly entities. For Correlational approaches, these affordances are used to bring new entities into being through the signs, marks and registrations of their effects. As we have seen, global warming, just like changes in body or air temperatures, can only be 'seen' via the registration of its 
effects, enabling everyday island interactions to become signifiers of planetary importance. Correlational approaches thus make islands important as mechanisms of perception, for seeing the world, for understanding the stakes of the Anthropocene. In ontoepistemological approaches, potentially all objects or entities can be made to 'speak to us' as their specific affordances register their worldly relationality. What entities have to 'say' depends upon their relations and affordances, and the potential for entities to 'speak' is only limited by our capacity to sense or see these. As we have already noted above, onto-epistemology can be taken further, and in the approaches of Storiation (as we will analyse in the next chapter) a more speculative approach is taken, which seeks to expand our capacity to imagine 'worlds' from other, non-human or more-than-human, perspectives.

\section{Notes}

${ }^{1}$ Like Grydehøj and Kelman (2017: 107), Godfrey Baldacchino (2020) has critiqued ways in which islands have been ordained 'as advance indicators or extreme reproductions of what is present or future elsewhere'.

${ }^{2}$ Perhaps the island artist who has done most on the international stage to explicitly foreground the trope of sensing the Anthropocene is the Icelandic artist Olafur Eliasson who has been 'inspired by Iceland to connect nature and art' (Skidmore et al, n.d.). In his 1993 work, Beauty, Eliasson created a darkened room with fine mist in it from the fallout out of a punctured hosepipe, which is illuminated by a single lightbulb; so that, from certain angles, the participant senses a rainbow. The whole point of this project, which leaves the lightbulb and hosepipe bare for the participant to see, is to explicitly draw the participant's attention to the importance of sensing itself: '... seeing yourself sensing. You'd not just be having an experience, but conscious of having that experience. You would be made self-aware by the set-up of his work, of that experience of looking' (Skidmore et al, n.d.).

${ }^{3}$ Such debates reorient the stakes of a critical tradition which has widely condemned how islanders are researched by Western 
academics and are co-opted into Western systems of knowledge and power (for example, Linda Tuhiwai Smith, 2012).

${ }^{4}$ As another example, Sophie Chao (2020) has worked with the Marind people of Indonesia to produce a multi-sensory map which examines how they correlate to their island differently from those who seek to develop oil plantations. Focusing upon Marind song, lands, vegetation, bird and animal life, Chao has produced a dynamic sensory map of shifting and flexible demarcations 'rather than a map of topography, or ownership, or territory'.

${ }^{5}$ See also Renee Pualani Louis and Moana Kahele's (2017) Kanaka Hawai'i Cartography: Hula, Navigation, and Oratory, which situates mapping in the island environment and encodes islanders' spatial knowledge into bodily memory via repetitive recitations and other habitual practices, such as hula.

${ }^{6}$ See Schneider-Mayerson (2017: 166) as illustrative of debates concerned with how Singapore's advanced sensing and adaptive technologies means that 'some islands will rise' in the Anthropocene.

${ }^{7}$ Anthropocene thinking is increasingly concerned with how debate is 'Trapped in all-too-human languages, sensual orientations, corporeal habits, graphic representations, and data visualizations' (Fish, 2019). Innovative work on islands is leading the way for alternative approaches to sensing and registering transformative planetary changes. Machine Wilderness (2019) is an experimental project developed by Theun Karelse, Alice Smits and a range of associates, involving sessions in the Venice Lagoon and elsewhere. By way of innovative symposiums, exhibitions, workshops and fieldwork sessions, this programme seeks to examine what sensing and correlational technologies would look like if they could directly relate to island environments in the way that organisms other than humans do. 'The Machine Wilderness program starts from the viewpoint of organisms (and technologies) as interacting populations surfing collectively on the geological and meteorological currents that carry them' (Machine Wilderness, 2019). Central to Machine Wilderness is the organising concept of 'biomes', long held as important to working with island ecologies and life. The aim is to develop new technologies which do not correlate and sense their surroundings like humans, but rather like communities of plants and animals which have correlated with relational forces and entanglements over time. 
${ }^{8}$ For example, radiocarbon dating has shown that Boot Canyon in Big Bend National Park had a very different environment fifteen to twenty thousand years ago than it does today. 'One result of this is the presence of so-called relict species that can live nowhere else in the park, such as big tooth maple, Arizona cypress, quaking aspen, and several species of oaks, which were stranded in Boot Canyon and Pine Canyon with the retreat of the last ice, far from their normal alpine habitats farther north in the Rockies and Sierras' (Wolfe, 2017: 139).

9 Adam Searle (2020: 169, emphasis in original) approaches the question of species extinction in a similar way: 'The ontological "presence-ing" of absence enriches us with ghosts, whom we should engage and allow to speak through their markings on the world. Only through learning to make sense of absences of not-there-anymore can we think through the absences of the future to come, of the notthere-yet. And this is thoroughly intertwined within an ethics and politics of the worlds in which we coinhabit, the ways we act in the present...'

${ }^{10}$ This approach accords well with Bruno Latour's claims for actor network theory, which inverts Marx's famous dictum in a way that clearly expresses the analytics of Correlation, that '[s] ocial scientists have transformed the world in various ways; the point, however, is to interpret it' (Latour, 2005: 42, emphasis in original).

${ }^{11}$ It should be emphasised that this turn to ontology is not about establishing a universal truth of how the world works but freeing epistemological approaches from modernist constraints, held to separate the subject from the world and to reduce the world to a narrowly human appropriation of it. 


\section{Storiation: Holding the World}

\section{Introduction}

In the previous chapter we examined how Correlational ontoepistemologies draw upon the capacities and affordances of islands in order to develop non-modern approaches to sensing and registering environmental change. Correlational onto-epistemologies work indirectly, grasping entities via the affordances of other entities upon which they are registered. Thus, they are dependent upon regular patterns of interaction and inter-relation, and operate to produce generalisable forms of calculation, measurement and comparison. An important analytical focal point on the other end of the onto-epistemological continuum is that which we conceptualise as Storiation. Central to the onto-epistemology of Storiation is how islands and islanders are engaged as registers of effects and relations in ways which are disruptive of modernist conceptions of space and time.

Often to the fore in these forms of knowledge generation are the ongoing afterlives and effects of such significant forces as global warming, nuclear radiation, waste production and colonialism (Sharpe, 2016; King, 2019; Barad, 2019; DeLoughrey, 2019; Farrier, 2019; Wang, 2020; Clark and Szerszynski, 2021). As we examine,

How to cite this book chapter:

Pugh, J. and Chandler, D. 2021. Anthropocene Islands: Entangled Worlds.

Pp. 141-178. London: Ubiquity Press. DOI: https://doi.org/10.16997

/book52.e. License: CC-BY 4.0 
Storiation registers this by employing tropes such as 'traces', 'the uncanny', 'quantum, 'speculation, 'hauntings' and 'spectres' (Morton, 2016a; Wolfe, 2017; Mathews, 2017; King, 2019; Barad, 2019; Neimanis, 2019). In Storiation, relations continue to reverberate in time and space in ways that are not separate from, but very much constitutive of, life in the Anthropocene.

This chapter is organised in three sections. The first section provides a detailed analysis of the underlying logic of Storiation. It foregrounds how work with islands is playing a particularly productive and generative role in the emergence and development of this onto-epistemology in Anthropocene thinking. The second section turns to Storiation work drawing upon islander life and island scholarship. The last section analyses what we call Storiation 'without the subject' which pushes further the speculative openings generated by island work and island imaginaries as productive of future and alternative possibilities beyond the world bounded by modernist or mainland epistemic concerns.

\section{The Analytics of Storiation}

One way to think about the importance of the onto-epistemology of Storiation is the following. In modernity, entities or products are available to us (for example, at a supermarket or on the internet) independent or autonomous from the relations involved in their production or marketing. They may have been produced through a multitude of different means and relations, with parts sourced globally and the final products perhaps manufactured far from the origins of their components (Read, [1958] 2019). If you are buying a mobile phone, for example, there is very little to indicate the components, raw materials and their sources, just as packaged food products do not reveal the stories behind their production on factory farms and slaughterhouses. In modernity, most entities that we come across are ones we find on supermarket shelves or are delivered direct to the door via internet sales - this means that the relational and feedback effects are lost to us (Kimmerer, 2013). This lack of access to or awareness of relational entanglement and its rationalisation in the modern episteme, 
is precisely what Anthropocene scholarship seeks to bring to light and to foreground (Yusoff, 2018; Cyphers, 2019; Arnall and Kothari, 2020; Sheller, 2020).

In the Anthropocene, feedback effects of human impacts become much more readily apparent, disrupting reductionist understandings which fail to track unintended or unexpected consequences. Authors are quick to stress that there is no 'away' and no past, there are no avenues for escape in the Anthropocene (Ghosh, 2016: 26; Morton, 2013). Modernist thought sees very little of this reality. Thus, Anthropocene scholars, drawn to working with islands as mediums of Storiation, seek to use feedback effects, marks, signs and registrations to provide greater access to this relational richness via speculative openings for thought (Marland, 2014; Morton, 2016a; Sharpe, 2016; King, 2018; Barad, 2019; Clark and Szerszynski, 2021; Perez, 2020a). Islands are understood as potential amplifying sites which hold differences and relations often in tension or contradiction: thus, the traces, hauntings and spectres disrupt easy separations between pasts, presents and futures.

David Farrier's (2020: 5-6) Footprints: In Search of Future Fossils provides an example of what we are calling Storiation as an approach. He opens his book with the famous example of Friday's footprint on the island in Daniel Defoe's Robinson Crusoe:

It happen'd one Day about Noon going towards my Boat, I was exceedingly surprizd with the Print of a Man's naked Foot on the Shore, which was very plain to be seen in the Sand: I stood like one Thunder-struck, or as if I had seen an Apparition; I listen'd, I looked round me; I could hear nothing, nor see any thing. There was exactly the Very Print of a Foot, Toes, Heel, and every Part of a Foot; how it came thither, I knew not, nor could in the least imagine. (quoted in Farrier, 2020: 5-6)

It is the particular message Farrier takes home from this passage which is important for clarifying the approach of Storiation we will develop in this chapter:

After enduring the solitude of his deserted island, he suddenly sees hints of human presence everywhere, 'mistaking every bush 
and tree, and fancying every stump at a distance to be a man' ... The discoveries of Friday's footprint and the footprints of early humans have such a vivid claim on our imagination because we have all lived a version of it at some point: the sudden feeling of being accompanied by an unseen other. Although you are alone, the air seems somehow closer, or an empty room is still thick with the presence of one only just departed. Someone or something has passed through already. (Farrier, 2020: 6)

Key for the analytics of Storiation is how older modern and Cartesian frameworks of reasoning adopted an impoverished ontology of presence, where any sense of 'withdrawal' is understood as an incidental feature of being. For commentators such as Farrier, we need a different onto-epistemology, or way of knowing, in the Anthropocene; where the footprints of humanity suggest that what can seem to be temporally and spatially distant or 'withdrawn' - such as global warming, waste production, nuclear fallout, the legacies of pollution, or colonialism - are also intimately 'close' and 'present.' In other words, 'The things we touch, touch us back' (Farrier and Dickenson, 2020). Here, Storiation seeks to materially reveal and register forms of relation and interconnection which modernist framings are necessarily blind to.

Storiation enables objects and events, which a modernist episteme would understand as over and done with, to hold and to exist for the present. We know from Marxism that the violence of primitive accumulation, dispossession and enclosure lives on under capitalism in relations of market contract, and the coercion of those without capital by those who possess it and can thereby put the labour of others to work. We learn from Foucault, that the violence of war lives on in the peace that is enforced by the state as a body that secures the circulation of power and interest despite the granting of rights and freedom. We know from Afropessimism that the violence of chattel slavery lives on in systems of liberal power which reproduce practices of white entitlement and black subordination and expendability (Hartman, 1997). In the Anthropocene, there is no 'away' and no 'over', no 'finished business' and no 'before and after'. The materiality of Storiation 
tells different truths about time, space and agency than the narratives and myths of a modernist imaginary.

Perhaps the most emblematic of all the islands of the Anthropocene is the Great Pacific Garbage Patch, a floating gyre of plastics, 'roughly the size of Texas, containing approximately 3.5 million tons of trash. Shoes, toys, bags, pacifiers, wrappers, toothbrushes, and bottles too numerous to count' (Alaimo, 2016: 130; Somerville, 2017). As Alaimo (2016: 130) states: '[e]veryday, ostensibly benign human stuff becomes nightmarish as it floats forever in the sea. The recognition that these banal objects, intended for momentary human use, pollute for eternity renders them surreally malevolent.' Objects and items can play fundamentally different roles - have very different lives and afterlives - but these cannot be separated from each other; the key point is that they are intimately connected in the analytical approach of Storiation.

Here we can see that unexpected and untimely afterlives may well be destructive rather than productive. But Storiation, even as 'death work', as Deborah Bird Rose stated (2011), is still productive of ways of thinking about how our actions are inserted into time and space in unpredictable and 'strange' or 'weird' ways. Thus, what were useful and reusable products assume other lives and other forms of agency as the detritus of consumerism, which, like colourful plastic bottle caps, pass from one more-than-human assemblage to another:

One bottle cap - such a negligible bit of stuff to humans - may persist in killing birds and fish for hundreds (thousands?) of years. There is something uncanny about ordinary human objects becoming the stuff of horror and destruction; these effects are magnified by the strange jumbling of scale in which a tiny bit of plastic can wreak havoc on the ecologies of the vast seas. (Alaimo, 2016: 130)

In Farrier's work discussed above, Storiation is not only marked by the idea that there is no 'away' in the Anthropocene, but also by the 'weird' and the 'uncanny' - footprints, hauntings, ghosts and traces of human and nonhumans: 'The Anthropocene binds together human history and geological time in a strange loop, 
weirdly weird' (Morton, 2016b: 8). Timothy Morton (2016b) opens his Dark Ecology with examples of the way that catastrophic climate change brings out connections in time and space beyond the apprehension of individuals as agents. For an individual shovelling coal into a steam engine in 1784 it is not easy to think about the material afterlives of the processes within which their action is embedded. In fact, it is impossible because these processes are perceived differently across relations in and of time and space. This action is both part of the initiation of the industrial revolution and also of anthropogenic climate change.

Events, no matter how brief or how small, through their relational embeddedness, can have infinite 'afterlives', with different paths, traces and entangled processes (Wolfe, 2017). As Morton highlights, this is not just true in time but in space as well. Even something as minor and statistically meaningless on its own, such as turning the key in a car ignition, when scaled up to include billions of key turnings, creates an agential force of huge environmental destruction. This gap between the given appearance of things, events or objects and their legacies and afterlives means that Storiation becomes a more important way of knowing through effects - than a positivist approach which understands objects purely at the level of the ontically given (Morton, 2016b; Farrier and Dickenson, 2020).

What we want to stress in this book is just how prominently the island - as a figure of relational entanglements in the Anthropocene - features for these contemporary approaches. ${ }^{1}$ Farrier's Anthropocene Poetics (2019), for example, critiques the modern utopian tech of Singapore island's famous 'Supertrees'; a combination of giant solar panels and vents for heat generated from the city's waste biomass:

Different expressions of human-inflicted deep time flow through the scene: in the panoply of products entombed in the containers, in the patina of carbon residue from the forest fires in neighbouring Borneo that might find its way into an ice core thousands of miles away, or in the soundless crashing of tropical diversity. The skies are clear, but in recent years, for much of June through 
October, the island has been enveloped in a thick smog blown in from illegal and uncontrolled fires, used to clear Indonesian rain forests and make way for giant palm oil plantations. The Garden City is a node in a vast network of extraction and consumption, linked to countless shadow places across the planet ... (Farrier, 2019: 126)

For Farrier (2019: 125-126), standing in the artificial canopy of the island's Supertrees is not a celebration of modernity, but is 'an encounter with a rather different kind of Anthropocentric moment ... It is a moment thickened by the collision of countless species' flight ways and intra-acting "stories of matter". Farrier focuses upon what we could call 'the thickness of the now' on the island, where the island registers countless material traces and hauntings of the past and protentions of a possible future. Farrier's work switches the register of attention away from concepts of graspable inter-relations, which could be coherently registered or read (as in Correlational logics), towards the interplay of material hauntings and traces of ongoing effects. ${ }^{2}$ What is needed is a 'defracted poetics' which holds together the traces, hauntings and afterlives of the relational 'entanglements' of the Anthropocene (Farrier and Dickenson, 2020).

The onto-epistemological approach of Storiation we find is particularly well expressed in Barad's (2019) work on the Marshall Islands, where island life is storiated via the effects of the atom bomb in 'quantum' ways. As in the work of Wolfe (2017) and Farrier (2020), for Barad, the island is a key figure for registering differences and relations of becoming; the traces of relations that destabilise a linear understanding of past and present. Barad (2019: 540) focuses upon the ostensible 'void' of the concrete insulating shield installed by the US military, in an attempt to contain and control nuclear contamination on the islands. But, for Barad (2019: 540-541), there is no 'away' in the Anthropocene and this concrete slab is:

A tomb inhabited by ghosts, material traces of the violence of colonial hospitality. The void as archive: the structured nothingness 
that is far from empty or de-void of meaning. This covering over, this attempt to dress up the naked infinities of the layering of violence upon violence, the incalculable brutality of superpositions of nuclear and climate catastrophes, the effects of militarism, colonialism, nationalism, scientism, modernism, racism, and capitalism, speaks to the specific structures of nothingness in their entanglement; in this case, a void within a 'void' at the 'end of the Earth' (in space) that signals the 'end of the Earth' (in time).

The island itself is an archive for speculatively storying the quantum nature of the world and the material traces of relations. Working with the already widely heralded relational affordances of islands in this way tells us that 'hauntings are material ... hauntings are the ontological re-memberings' (emphasis in original), and that what 'the world calls out for is an embodied practice of tracing the entanglements of violent histories' (Barad, 2019: 539). Central here is also Barad's attention to the artifice of modernist constructions of space-time as a container for the causal interaction of already existing entities. ${ }^{3}$ For this reason, she stresses the importance of intra-, rather than inter-activity for the generation of alternative ethico-onto-epistemological accounts and understandings.

Intra-action clarifies that the cuts and separations between subject and world, the distinctions between entities and those separating the past, present and future are products of the human or subject-centred modernist episteme, rather than their pre-existing relation. When Barad talks of justice or responsibility to what she speculatively stories about islands, the key point she makes is that it is impossible to separate the materiality of islands themselves from the human subjects speculating upon them. Storiation is a process of infinite depth and possibility, in which the quantum level holds, suspends and pre-exists the cuts and separations imagined by a modernist epistemology of fixed grids of time and space. ${ }^{4}$ Thus, approaches of Storiation engage islands to disrupt or put a break on those who would seek to more coherently grasp or know a world imagined to be framed in separated and segmented grids of time and space. 
Key to the knowing of the disruptive onto-epistemology of Storiation then is that there is much less emphasis on temporal lines of 'progress' or increasing individuation and complexity. Approaches to Storiation invoke this particular interest in and imaginary of islands as key sites for understanding relational entanglements. In Storiation, everything is always already here in quantum superpositionality - meaning that Storiation works as a 'hold' in two ways. Firstly, as a way of holding or keeping together aspects that are separated or cut in order to be brought into or actualised for a modernist episteme. Secondly, Storiation works as a hold or a barrier to the modernist demands that everything should be revealed, so that it can be 'known' in accessible or instrumentalisable ways that can then be generalised or scaled up. Islands thereby are imagined speculatively and worked with to produce more 'chaosmotic', less systematising and ordering approaches. A question which might arise at this point is why disruption is seen positively, rather than merely as a destructive or negative force, in the analytics of Storiation? The response is that we need disruption. If humanity is responsible for the 'death work' of the Anthropocene - the forces of nuclear fallout, global warming, rising sea levels, the ongoing legacies of colonialism, and other transforming conditions - then we need to somehow grasp what it means to be an entangled being.

Morton's Dark Ecology (2016b) develops an approach we would also frame through the analytic of Storiation. To see our relationally entangled being - the starting point for ecological thought, for Morton - we need to step outside the problem-solving universalist 'one world' mind-set which is so essential to modern frameworks of reasoning. Morton (2016b) explains that, as individuals, we appear in many ways; as parts of associations, families, professions, states etc., including as part of a species with species-effects. None of these manifestations is under our control - even our appearance to ourselves or to friends or lovers: every manifestation is entangled within many other networks of interconnection with emergent effects, yet we contribute, intentionally and unintentionally, to all of them. 
Whilst this can appear rather abstract when discussed in this way, as we have seen, such approaches are given particular purchase through how the figure of the island is put to 'work' to ground the development of Storiation as an onto-epistemology or way of knowing in the Anthropocene (Morton, 2016a). Here global warming is understood to unfold through vast spatial and temporal forces - from the more immediate and intensified violence of hurricanes on a small island, to large-scale sea level rises, complex changes in atmospheric conditions, and the hundred thousand years or so it takes for increased levels of carbon to dissolve in the surrounding oceans. Yet, at the same time, all islands in the world already exist within global warming, there is no isolated island, just over there, beyond the horizon (Morton, 2016a). Global warming is thus what Morton calls a 'hyperobject': both intimately close and infinitely withdrawn, in that we cannot literally see it beyond its presence via its effects. If we can understand islands as part of such strange and shifting manifestations or appearances, all partially 'looped' or interconnected, and operating across infinite scales of time and space, then, for Morton (2016a), we can begin to see what it might mean to think and work in ecological rather than modernist ways.

Leading Anthropocene scholars like Wolfe, Farrier, Barad and Morton regularly turn to islands as significant figures for helping us work through the central problematic of relational entanglements in the Anthropocene. Morton's (2016a) island essay Molten Entities begins to do this by drawing upon the work of the widely acclaimed Icelandic artist Olafur Eliasson, who displayed a large block of ice from Greenland at the Paris Climate Change Conference (COP21). Using this work, called Ice Watch:

Eliasson was hoping to show how the ice invites us humans into something like a dialogue or dance. The ice is not simply an unformatted surface waiting for us humans to make it significant. The molten edges of the ice block, displaced in a Paris square, become a way to think about how beings are intrinsically in motion because they are intrinsically melting, fragile. (Morton, 2016a: 71, emphasis added) 
For Morton (2016a: 71), Eliasson's high-profile artwork reveals how we can approach the 'ambiguous edges' of entities in the Anthropocene, rather than assuming entities as neat and selfcontained essences prior to relations in spatially extended assemblages and networks. As Morton says, whilst the 'idea that "No man is an island" is obviously very popular right now as a progressive concept' (2016a: 71), 'Relating isn't some wondering way to fasten islands into chains to make them more exciting. Relating is how a thing is, all by itself' (2016a: 73, emphasis in original). For Morton, working with islands is an invitation to think differently, to see entities as infinitely relational, 'all the way down':

If you look at the coastline of an island from space, you will see something fairly regular - perhaps it's rather triangular. When you look close up, say from a hang glider, you will see all kinds of curves and folds that you didn't see from space. And when you crawl around the surface of the coastline as an ant about three millimeters long, you will find something very different again not just impressionistically different, but extensionally different: the circumference will be a different length. Indeed there may be circumstances - ways of measuring that island - that cause its circumference to be infinite. This is rather like what happens when you examine something like a Koch Curve, the fractal shape in which triangles are populated with smaller versions of themselves to infinity. One ends up with a shape that is bounded yet infinite. The Koch Curve is strangely 'more than itself' at every point. An island is a cornucopia, or TARDIS, that contains more of itself on the 'inside' than it appears to have on the outside. This is because they always exceed how they appear, even to themselves. They melt out of themselves, without moving in space or time and without being pushed by anything. (Morton, 2016a: 71-73, emphasis in original)

Morton thus switches the register of island imaginaries by way of Storiation to speculate beyond the impassable rift which opens up between island relations in their ontological reality and the capacity of humans to register this totality. ${ }^{5}$ Of course, there has been a much longer history in which islands have often been thought of as sacred spaces for speculation; the difference being that, 
for Anthropocene scholars like Morton, 'sacre' (Gillis, 2004: 26, emphasis in original) now does not mean a place that is separate or apart. Contemporary debates are not about isolated and insular islands that people can escape to, away from the overwhelming busy-ness of the world; they are precisely the opposite. Islands, as sites of relational entanglements, can develop our awareness that we exist within vast multidimensional forces, such as global warming, which 'means to approach, then diminish, from a certain fullness' whose total reality is fundamentally inaccessible to humans (Morton, 2013: 74).

If reality is withdrawn and we only perceive signs, signals or effects, never reality itself, then this can appear to make Storiation similar to Correlation, except that, for Storiation approaches, the signs are not indicators of fixed or stable relations and therefore open up, rather than close down, speculative possibilities. ${ }^{6}$ This point is crucial for drawing out the analytical distinctions between Correlation and Storiation. For Correlational ontoepistemologies, signs require stable assemblages of other actants to maintain them. As examined in the last chapter, it is these regularities which enable Anthropocene thinking to work with island life as an important way of registering underlying planetary changes. This is not the point for Storiation approaches. As Morton argues, every ant sees differently, every hang-glider and every grain of sand on the beach of an island is individual. For Storiation approaches then, Correlational insights turn out to be just as reductionist and essentialising as linear causal imaginaries. ${ }^{7}$ Thus, Storiation approaches that work with the evolution of island life instead turn their attention to:

the spectral presence of evolutionary time inside the bodies of organisms. Every new species inherits parts of its body plan from earlier organisms. For those who want to admire the diversity of life, the trick is not to imagine this inheritance as teleological progress, the climbing of a ladder toward the sun. Instead, we might appreciate the ghostly presence of ancestors inside us, which makes it possible for us to do whatever we do. (Tsing et al, 2017: G65; Hejnol, 2017) ${ }^{8}$ 
Evolution on islands appears not as a teleology of increasingly complex inter-relational efficiencies which can be coherently read or registered (as in Correlation), but rather as a carnival of openended potential. ${ }^{9}$ Hauntings, traces and afterlives reverberate in ways which open out to multiple and simultaneous becomings. ${ }^{10}$ Storiation is closely linked to the 'ontological turn' in disciplines such as anthropology, where the focus is taking ways of knowing beyond human subject-centred approaches (Holbraad and Pedersen, 2017). Thus approaches of Storiation, involving 'speculation' and 'speculative bricolage', often draw upon the experience of being within distinct 'islands' or related ecosystems, widely held to facilitate such understandings. ${ }^{11}$ As Andrew Mathews (2017: G145) says about the forest ecosystems he explores in Monti Pisani, Italy:

Through my practices of walking, looking and wondering, I have been tracing the ghostly forms that have emerged from past encounters between people, plants, animals, and soils. The ghostly forms are traces of past cultivation, but they also provide ways of imagining and perhaps bringing into being positive environmental futures.

In the Storiation approaches with islands of Farrier, Wolfe, Barad, Morton, the examples we have placed in the notes of this chapter, and here, Mathews, we have a way of approaching the Anthropocene which is not about:

describing the relations between pregiven entities but rather of attending to the multiple forms that emerge from partial relations between different plants, animals, and people. [For example, a] chestnut is not one thing: it can be a gnarled ancient tree that is in a set of partial relations with goats, people, sheep, and terraces; a chestnut can also be a dense forest of pole-sized stems of 'wild' coppice/ceduo, cut repeatedly to produce firewood for local household consumption or perhaps to produce woodchips for biomass energy plants that produce electricity. New diseases may change social relations, but these diseases may themselves change, as in the transformation of chestnut cancer into its hypo- 
virulent form ... Paying close attention to the ghostly forms of past histories in present-day forests allows us to consider the many forms of political and economic life that these forests are or might be connected to, including imagining multiple possible Anthropocene futures. The texture and form of our material surroundings are full of speculative politics and causal accounts ... (Mathews, 2017: G153-154) (2 $^{12}$

In such scenes, which speculate upon simultaneous and multiple becomings and ghostly presences, the language of 'feedback effects' fails to capture how, as Mathews points out, entities do not pre-exist feedback effects or communication but, in fact, constitute them. To think in terms of entities adapting to others via feedback, or to pose the problematic in terms of Bateson's (2000) cybernetic framing of 'organism plus environment', would fail to fully grasp Storiation as a speculative approach. For Storiation, the materiality of the world is the starting point for thinking differently about the traces, afterlives, ongoing effects and legacies of modernity in 'weird' (Morton), 'quantum' (Barad) and/or 'haunting' and 'ghostly' (Farrier and Mathews) ways.

\section{Storiations: Holding the World Together}

'Storiation' is a particular way of approaching the Anthropocene as a problematic which focuses upon how legacies, hauntings and ongoing effects enable the materiality of the world to open possibilities for thought. In many older, particularly European, storytelling traditions, when someone 'tells a story' they are the storytelling subject applying their interpretation of the world, and often drawing out a moral lesson from an object or event. Storiation seeks to do something quite different: to speculatively enable the world to 'speak' or narrate stories. However, Storiation is much more than a shift in subject position. The speculative products of Storiation seek to hold more of the world together, making the world more real, less segmented, cut and divided. 
Here the island, both as a significant figure of relational entanglements in the Anthropocene and as liminal space or 'outside' of modernity, plays a particularly important and generative role for the development of Storiation approaches. This can be seen particularly effectively in Barad's attention to the quantum as a speculative realm holding together on islands what modernist epistemologies actualise by cutting and separating. Subjects and objects, and time and space are products of these cuts: they do not pre-exist them. Thus, Storiation draws attention to the violence of modernist epistemic knowledge and is thereby '(re)Storiative', holding together that which modernity seeks to cut and separate, disrupting assumptions of segmented time and space.

Elizabeth DeLoughrey's (2019) book, Allegories of the Anthropocene, foregrounds feminist approaches operating within the Storiation analytic to emphasise the material experience of island existence. Thus, DeLoughrey is concerned with redressing the violence of the modern episteme through her focus upon (re)Storiation by holding, or 'telescoping' (DeLoughrey, 2019: 2) together, that which modernity seeks to divide or to cut out. ${ }^{13}$ In developing her approach, DeLoughrey $(2019,192-192)$ is drawn to the work of Marshallese poet Kathy Jetñil-Kijiner, who became 'an international celebrity since her moving performance at the opening ceremony of the United Nations Climate Summit in New York in 2014'. Jetñil-Kijiner's work is often held to exemplify islands as spaces that hold together forces of colonialism and climate change (see Faris, 2019; Perez, forthcoming). DeLoughrey examines how her poetry 'employs allegory to figure the island as a world in ecological crisis, depicts an active, nonhuman ocean agent, and articulates the imperative to both witness and testify to a dynamic, changing Earth' (DeLoughrey, 2019: 1). Thus, in the poem 'Tell Them' Jetñil-Kijiner says, 'Tell them what it's like/ to see the entire ocean_level_with the land' (quoted in DeLoughrey, 2019: 1). As DeLoughrey (2019: 193) points out, here Jetñil-Kijiner's work 'does not employ an aerial, god's-eye view of the tropical island', one which segments time and space, in a modernist way. On the contrary, her poetry holds, or telescopes together, how the 
violence of this god's-eye view is constitutive of the everyday, ordinariness of climate change for island life.

Elaborating the point, DeLoughrey (2019: 194) discusses how Jetñil-Kijiner offered the United States a gift in the form of handcrafted jewels taken from the ocean and 'placed in hand-woven baskets, products of women's love and labor. Inside this basket is a message, which is an allegory for the poem that we are asked to pass on'. Here iep jeltok, in Marshallese culture, 'signifies a basket facing toward the speaker, foregrounding material and cultural exchange in a matrilineal society. The basket is also a major figure for Indigenous women's artistic labor and about weaving together connections and obligations across the Pacific' (DeLoughrey, 2019: 193). It is 'in keeping with Indigenous socialities in Oceania in which, as Marilyn Strathern and others have demonstrated, the center of the social network is not individual but relation itself' (DeLoughrey, 2019: 194). Thus, the act of offering the basket and the poem can be seen as a feminist, (re)Storiative act - it holds together the disjuncture between the god's-eye view of islands in the Anthropocene (dominated by the Western, White male) and the ordinary experience of climate change. Key to this act, for DeLoughrey, is how this disjuncture is not presented as separated from, but as constitutive of, everyday experiences of island life.

In Planetary Social Thought: The Anthropocene Challenge to the Social Sciences, Nigel Clark and Bronislaw Szerszynski (2021) similarly draw heavily upon the work of another island writer, Katerina Teaiwa $(2011,2012,2015)$, concerned with the mineral phosphate mining which has devastated the Kiribati island of Banaba. For Clark and Szerszynski (2021: 146), these islands are 'paradigmatic of the "blast: dump: crush: extract: exhaust" modern mentality'. Clark and Szerszynski are particularly interested in how the ongoing traces, hauntings and legacies of this mining are registered, intra-relationally, in contemporary islanders' embodied movements and dances. Key to this, for Clark and Szerszynski (2021: 146), is how, 'like most other Pacific societies, human life is inseparable from land, just as land and sea often merge into one another'. It is this which opens up the possibilities for Storiation. 
More specifically, for these islanders, 'the concept of te $a b a$, or land, in the Kiribati language spoken by both I-Kiribati and Banabans unites the body of the land with the bodies of the people' (Teaiwa quoted in Clark and Szerszynski, 2021: 146).

As Teaiwa says, ' $\mathrm{t}$ ]he body of the people is in that landscape so when it's mined and crushed and dug up, you're not just doing it with rock, you're also doing it with people, with the remains of people' (Teaiwa quoted in Theobold, 2018, and in Clark and Szerszynski, 2021: 146). For Clark and Szerszynski, this provides us with a powerful way of 'knowing' the Anthropocene - what we call Storiation - where the ongoing legacies and traces of modernity and colonialism are registered, intra-relationally, in these islanders' embodied movements, dances and performances. These dances are not stories 'about' islander life; they 'Storiate' through their embodied and bodily movements. As Clark and Szerszynski (2021: 164-165, emphasis in original) point out:

Thinking, writing, speculating through the Earth, however, does not come easy to those of us who fledged in worlds that were constituted with the very aim of raising the subject high above any merely material threat to its continued flourishing. It is in this sense that the proliferating earth-beings, telluric spirits and animate objects of Indigenous worlds offer incitements to western thinkers: not just through their demonstration that other ways of composing realities are equally feasible, but because they offer practical lessons in enduring or thriving in the thick of lifethreatening threshold events.

In the analytic of Storiation, islands and islanders are holding or amplifying sites which foreground the violent work of the modernist episteme; but are also (re)Storiative of new possibilities, holding together that which modernity seeks to cut out and disavow. Thus, for authors such as Mimi Sheller (2020), Emanuela Borgnino (2020) and Tamara Searle (2019), the (re)Storiation of island life in the Anthropocene not only requires rejecting modern ways of 'grasping' the world, and linear imaginaries of progress and development, it also requires opening up understandings of 
islands to more speculative possibilities when routed through islanders' own belief systems and practices. Emanuela Borgnino (2020) charts her Storiation of the ongoing legacies of colonialism and environmental degradation on O'ahu, Hawai'i, through Indigenous understandings of land ('äina), and Tamara Searle (2020), on Suomenlinna (Finland), through the Saami peoples' folk beliefs relating to land and spirit. In Island Futures: Caribbean Survival in the Anthropocene, Sheller (2020) charts her Storiation of Haiti and Haitian life in the Anthropocene by engaging Vodou loa and songs, shamanistic and African-rooted traditions where spirits come into people's bodies, through such practices as dance, music and trance.

As Sheller (2020: 146) says, '[o]utside the genres of academic social science, beyond the conventions of individualistic thinking and linear narrative, without the closure of monotheistic biblical time horizons where redemption await us, the Vodou loa interrupt the scene of human hubris.' They 'remind us that there are indeed other ways of affectively registering the ongoing disaster of human existence' (Sheller, 2020: 146). But to be clear, for the analytic of Storiation, and in the work of Sheller, this is not about generating stories 'about' island life, in the modern sense of the storyteller noted above. Rather, islander life itself, and islander practices, become the mode, force, or pathway of Storiation; which holds or telescopes together that which modernity seeks to cut out, separate and disavow. Thus, Sheller draws upon Kamau Brathwaite's notion of 'psychic marronage', in which Caribbean people maintain alternative patterns, symbols and practices, including 'modes of walking, eating, working, interrelating, musical, artistic, and other [cultural] practices' (Brathwaite, quoted in Sheller, 2020: 152). Islander life and ways of being become key sites of alternative, speculative possibilities and ways of knowing in the Anthropocene.

As mentioned in Chapter 1, Kamau Brathwaite has been particularly influential for those working within this analytic (Sharpe, 2016; Hessler, 2018; King, 2019). When Brathwaite (1999: 34, emphasis in original) informs us about the Caribbean woman sweeping the sand on the beach, he understands that colonialism 
is 'held' in her embodied actions - in 'our nanna's action, like the movement of the ocean she's walking on, coming from one continent/ continuum, touching another, and then receding ("reading") from the island(s) into the perhaps creative chaos of the(ir) future...'. What is at stake in Brathwaite's Storiation then is the working through of intra-, rather than inter-, relations; colonialism and the island condition register as her embodied movements as she sweeps. Brathwaite (1999: 34, emphasis in original) famously employs the term 'tidalectic' to describe this islander psyche which is a product of colonialism and the island condition. He goes to great lengths to distinguish tidalectics from European frameworks which always centre the human subject as storyteller. Thus, Brathwaite's is not a story about an islander; it is a Storiation which explicitly seeks to work with and think from her islander life and embodied movements themselves; how

[the] 'meaning' of the Caribbean was in that humble repetitive ritual action which this peasant woman was performing. And she was always on this journey, walking on the steps of sunlit water, coming out of a continent which we didn't fully know how to understand, to a set of islands which we only now barely coming to respect, cherish and understand. (1999: 33-34)

Braithwaite's approach helps us to think through the onto-epistemology of Storiation, an analytic which we read as important for many writers in contemporary Black Studies. These include Christina Sharpe (2019), whose work focuses upon Black lives, lived in the wake of colonialism, 'holding' together 'histories and presents.' Sharpe's (2016) highly influential book, In the Wake, engages Brathwaite as a key figure for registering the hauntings, traces and legacies of colonialism intra-relationally. Sharpe (2016: 34) quotes Brathwaite's Dream Haiti about contemporary Haitian refugees at sea: 'The sea was like slake gray of what was left of my body and the white waves ... I remember. ${ }^{14}$ For Sharpe (2016), as for DeLoughrey (2010: 708), Brathwaite's material registration is a powerful depiction of 'a collapse of the space and time separating the contemporary interdiction of Haitian refugees 
at sea and the long history of patrolling African bodies in the Middle Passage.'

The past is not over, as in the linear temporalities of modernity, but is constitutive of the islander present; registered, intrarelationally, as the Black bodies of the Haitian refugees. ${ }^{15}$ For Sharpe, we must reject modern frameworks of reasoning as a way of understanding, or grasping, these bodies. They should instead be registered, or known, onto-epistemologically, as holding spaces of speculative possibilities. Sharpe (2016: 76) is explicit on the matter. Her understanding is that for Brathwaite, as for the more recent work of Fred Moten (2003), the inability to register Black bodies by way of modern frameworks of reasoning is testament to the fact that objects can and do resist' and 'blackness - the extended movement of a specific upheaval, an ongoing irruption that anarranges every line - is a strain that pressures the assumption of the equivalence of personhood and subjectivity' (Moten, 2003: 1; quoted in Sharpe, 2016: 76). This is highlighted in Brathwaite's poem Dream Haiti, where 'a Coast Guard cutter becomes, in Brathwaite's hands, a Coast guard gutter - not a rescue or a medical ship but a carrier of coffins, a coffle, and so on. As the meaning of words fall apart, we encounter again and again the difficulty of sticking the signification. This is Black being in the wake' (Sharpe, 2016: 77, our emphasis). For Sharpe, the Black islander's body is not only a powerful disruptor of modern reasoning, it is also a 'holding' space of new speculative possibilities. ${ }^{16}$

The work of Moten in critical Black Studies, just noted, is also heavily influenced by Caribbean island writers, and here Jackie Wang (2020) has astutely drawn attention to 'Moten's Seaborne Sociality': 'a para-ontological mode of being that is literally connected to and produced by the ocean', where, in the Middle Passage, 'the sea is that which unsettles being' (Wang, 2020). Blackness is thus 'figured as a passage that marks ontological rupture, and 'is oceanic insofar as it is not fixed to that particular land base but unsettles the notion of home and is marked by dislocation' (Wang, 2020). Blackness then, 'is an uncoded zone of being ... a condition of possibility for the creation of ... undercommon sociality' 
(Wang, 2020). The sea and the middle passage are 'used to theorize the fluidity of blackness' - an experience that 'exhausts description' and 'deconstructs notions of the subject as bounded' (Wang, 2020). Whereas the onto-epistemology of Correlation, discussed in the last chapter, suborns or subordinates knowing to sensing and registering regular patterns of inter-relation, Storiation is by contrast more liberating; not in the sense of radical or revolutionary political practice, but in the sense of exploring and speculating upon the reality that we are already in: 'I believe in the world and want to be in it. I want to be in it all the way to the end of it because I believe in another world in the world and I want to be in that' (Harney and Moten, 2013: 118, emphasis in original).

Storiation is affirmative work in the world we are in already; rejecting modern frameworks of reasoning and speculating upon the embodied intra-relational movements that are to hand. For Harney and Moten (2013: 130), this is 'prophetic' work but only in the sense that it is about 'enriching being' rather than 'flattening' it. Storiation approaches provide a relation of depth, of immersion into the world: 'it's just a way to think about the already-existing enrichment of being, the already-social quality of time and space' (Harney and Moten, 2013: 130). This makes the world more 'generative', more real and more strange and unstable, than the flattened, or abstract, lifeless world of a modernist or universalist ontology, or that of Correlational onto-epistemologies. Thus, the onto-epistemology of Storiation is about revealing another world in the world, one which is much more obviously unmoored from or ungrounded in modernist frameworks of reasoning.

Another example of how the approach of Storiation could be illustrated is Tiffany Lethabo King's (2019) The Black Shoals. King's concept of the 'shoal', as in Brathwaite's 'tidalectics', is 'simultaneously water and land' (2019: 28); which, when contrasted with modernist continental/oceanic divides, 'presents a site of conceptual difficulty' (King, 2019: 28). The 'shoal represents a process, formation, and space that exists beyond binary thinking' (King, 2019: 28) by registering the intra-relational praxes of Black and Indigenous peoples living on in the wake of slavery and the 
legacies of colonialism. King (2019: 28-29) provides many illustrative examples, such as:

where Black and Indigenous speech and grammar share the same tongue; where Black and Indigenous resistance disrupt the master codes and cartographic representation of Man on an eighteenth-century map; where Black porous bodies tell histories of Black and Indigenous survival in 'uninhabitable zones', where Black and Indigenous erotics force an unmooring of the self; and where decolonial aesthetic practices sculpt new epistemologies and sensibilities that shape the contours of humanness in more expansive ways. The shoal offers an analytical site where multiple things can be perceived and experienced simultaneously.

At the heart of King's (2019: 207) conceptual framework of the 'shoal' is how modern, mainland, continental thinking does not enable the Storiation of Black or Indigenous resistances: 'Rarely does land evoke the kind of flexibility, elusiveness and tricksterlike qualities that Black diasporic life symbolizes in the Western hemisphere.' Thus, it is by turning to other strands of scholarship, such as Brathwaite's island poetry, holding together land and water simultaneously, that we are able to 'think about dynamic, fluid, and ever moving Black diasporic subjectivity' (King, 2019: 207). For King, it is Brathwaite's focus upon embodied, intra-relational becoming which is absolutely central here. In turn, like Sharpe, King argues that we should not seek to grasp or tell stories about Black bodies, but rather speculate from their embodied movements. This is key to enabling us to more effectively register the legacies, traces and hauntings of colonialism, and to therefore:

challenge forms of what I am calling 'applied intersectional frames' that attempt to discover, connect, or wrangle together experiences and power dynamics that are conceived as emerging independently of one another. The conceptual tools of 'discovery' assume a binary that must be overcome or discrete phenomenon that must be connected in ways that occlude their co-constitution or oneness. Part of the methodological contribution of The 
Black Shoals is its attunement to and disruption of the binaries and chasms that are overrepresented as an epistemological truth. (King, 2019: 28, emphasis in original)

The Black Shoals is a good illustration of Storiation as a speculative approach. There is no reading of correlations to see entities and forces which can be appropriated in modernist, subject-centered frameworks of understanding. The focus is upon drawing out multiple ways of knowing what is, and, like others noted above, King (2019: 29) explicitly employs 'critical fabulation' and 'speculative bricolage' in order to effectively hold together the traces, hauntings, ghosts and afterlives which enable us to see more and differently in the present; to include entities and relations which would not be noticeable if we constrained ourselves to reductive, modern, linear epistemological frames of space and time. Thus, King (2019: 30) develops her approach:

By assembling, shoaling, and rubbing disparate texts against one another, unexpected openings emerge where different voices are brought into relationship. As new relationships among texts and voices are made, new and 'transgressive ground[s] of understanding' emerge where one can begin to notice where rupture and 'momentary dislodgings' reveal that the archive is not a closed system that contains only one story. ${ }^{17}$

The Black Shoals has been influential for the development of island studies in the Anthropocene. Rebecca Schneider (2020: 201), for example, draws upon King's method in order to (re)Storiate, speculate and undertake a critical 'fabulation' of the 1803 'Igbo Landing' on St. Simons Island, Georgia, 'in which a group of enslaved Africans mutinied against their captors and ran aground upon a shoal.' Following King, Schneider (2020: 201) 'explores not only the littoral fact of shoals in seafaring but also the concept of shoaling for troubling historical narratives oriented to settler colonial plot points.' As a performative piece of writing, Schneider's (2020: 201) work 'shoals together' such considerations as accounts of sand, drift and 'accounts of Africans who fly' associated with 
this event. This not only works to disrupt modern frameworks of reasoning, a linear telos and the temporalities of colonial progress, but approaches the island and 'Igbo Landing' as a holding space for (re)Storiation and the generation of speculation possibilities. Thus, Schneider (2020: 201) does not draw out a singular argument or tell a story 'about' the island, but rather explores, shoals and rubs together 'the littoral zones among and between ideas, stories, arguments, facts, and fabulations in relation.' ${ }^{18}$

Although the empirical focus of King and Schneider's work is different to that discussed earlier, of Mathews (islands of forest ecosystems), Barad (nuclear fallout on islands), Morton (developing ecological awareness from thinking with islands) and Farrier (the reverberation of the human footprint on an island across space and time), what they share is a central analytical interest in speculative approaches which emphasise that thought can move beyond the limits and constraints of modernist forms of representation. Thinking with islands, islanders and/or certain island writers, becomes a very important way of drawing out these concerns and developing these particular approaches. The reason for this is that islands, islanders and the writings of island scholars like Brathwaite, Teaiwa and Jetñil-Kijiner, discussed above, foreground the liminal 'outside' of modernity; which is today being repositioned as central for moving beyond the limitations of modernist understandings of ontology and epistemology.

These Anthropocene approaches seek to make the world more alive and real to us by holding and including ongoing effects and legacies, traces and hauntings inaccessible to modern thought. What was excluded from modern mainland thinking - consigned to the past or excluded from linear and reductionist causal relations - is included and brought close and present in Anthropocene thinking. For many contemporary commentators examined in this chapter, the end of the modernist imaginary means that we need to reorient our empirical focus towards a much richer reality. To illustrate this, geographical forms such as islands, and the works of many island scholars, appear readily available for working in speculative ways. If modernity is to be rejected then, the 
argument goes, we must adopt other, more sophisticated, speculative methodologies approaches, foregrounding other ways of generating knowledge.

\section{Storiation Without the Subject}

The main contention of this book is that Anthropocene thinking frequently draws upon particular island imaginaries, island scholars, poets, artists and activists to engage the key problematic of relational entanglements, awareness and/or feedbacks, and for the stimulation or understanding of different ways of being and knowing. The continuums we have heuristically presented emphasise the development of relational or immanent approaches to ontology (Resilience and Patchworks), and onto-epistemological approaches to the production of thought and meaning (Correlation and Storiation). These illustrate a trend of thought which moves ontological and epistemological approaches increasingly further from modernist assumptions.

Perhaps this is most obvious in the analytic of Storiation, where the modernist distinctions, cuts and binaries which reductively grasped island life are erased in the work of Anthropocene thinking, and islands become worlds 'in-difference'. By this we mean, as we have said above, that islands become figures whose relationalities are too vibrant to be cut into, or grasped, by modern forms of representation - such as regular relations or coherent boundaries (Teaiwa, 2011; Morton, 2016a; Mathews, 2017; Sharpe, 2016; Barad, 2019; King, 2019; Clark and Szerszynski, 2021; Perez, 2020a). As Burgos Martinez (2020; forthcoming) further reflects on the example of 'small' Indonesian islands 'how can one even begin to understand the convergence of many natures ... when "the island" becomes a place needing to be translated ... ?' This point, which earlier island scholars such as Glissant (1997) have made - that island life is too rich, too vibrant, to be grasped by modern frameworks of reasoning - has become productive for more contemporary debates in the Anthropocene as islands are increasingly understood as a world of potentialities, yet to be fully realised 
or actualised. Our argument is that work with islands, islanders, certain strands of island scholarship, poetry, art and activism does some of the most important work for the development of these debates, and for the analytic of Storiation; not only in the influential scholarship of Karen Barad, Timothy Morton and David Farrier, but also in the work of critical Black Studies scholars, such as that of Christina Sharpe and Tiffany Lethabo King.

Perhaps it is in the field of anthropology that speculative approaches have been pushed furthest. Working on the basis of the 'conceptual affordances present in a body of ethnographic materials', Holbraad and Pedersen (2017: 294) argue that speculative analytics 'imply a peculiarly non- or anti-normative stance'. Rather than a means to externally defined political ends, Holbraad and Pedersen (2017: 295) claim that this approach is 'a political end in its own right'. This is because speculative approaches of Storiation attempt to bring the world to life on its own terms and are 'oriented towards the production of difference, or "alterity", as such' (Holbraad and Pedersen, 2017: 296). They (2017: 211) state that instead of starting from the desire to know or to represent 'things': 'Rather, the strategy must be one that is capable of effectively de-theorising the thing, by emptying it out of its many analytical connotations, rendering it a purely ethnographic "form" ready to be filled out contingently, according to its own ethnographic exigencies.' This 'is the prime step towards allowing things to dictate their own terms of analytical engagement' (Holbraad and Pedersen, 2017: 211). Allowing the thing or entity to speak, while minimising the role of the human interpretant is thereby an ethico-political goal in itself.

This reworks the anti-mainland or continental mantra of island studies: 'islands speaking on their own terms' (McCall, 1994; Walcott, 1998; Baldacchino, 2008; Depraetere, 2008). Rather than reducing (island) life via abstraction, Storiation aims to render life in richer, more concrete (Holbraad and Pedersen, 2017: 235), ways through speculating upon how things or artefacts 'analyse themselves', inviting new trains of thought (Holbraad and Pedersen, 2017: 234). Here, Storiation as onto-epistemology without 
a representational subject begins to turn islands and island practices into a world that cannot be an object. If the goal of speculative work is to remove the knowing and meaning-making subject - to use the appearances of the world to decentre the human - then the logical conclusion is that island experiences should imagine the subject with/in-difference. The subject in the world without cuts, binaries and distinctions. The subject in the world without appropriating objects to then be placed in grids of time and space.

The 'return' of the human to the world in this way would speculatively produce in-difference - neither a new telos of problem-solving awareness of how to manage and direct the world differently, nor a narcissistic feeling of being at one with the world of relational care and enablement. Claire Colebrook (2019: 175) writes:

Rather than think of distinct essences and fixed beings, we now acknowledge that nothing is an island; we - and the things around us - become what they are through encounters, with encounters and relations generating an openness, fluidity and dynamism of life and the world ... [But this can lead to a] moralism that is embedded in a complex metaphysical, aesthetic and theological history that privileges becoming and relations over the horror of something that simply is, bearing no relation to anything.

Colebrook (forthcoming) powerfully articulates the island withdrawn from the world of meaning; the island with/in-difference. If Storiation approaches seek to speculatively leave the subject behind, giving 'what is' or alterity its due, and recognising that the world cannot be reduced via modernist forms of abstraction and representation, then Storiation has to work to keep everything together without cuts, without re-presentation. What would it mean to understand an island, or indeed an islander, as in-different, completely withdrawn and not available for 'us' in this way? Whilst this goes further than many of the approaches examined so far, this is the question we focus upon in the conclusion of this chapter because it represents a logical end-point to Storiation as a speculative analytics that seeks to give onto-epistemological priority 
to matter/ing rather than to the thinking subject. The end point for an ethico-political duty of care, which pays attention to the world rather than starting from the needs, interests and desires of the subject.

We suggest that Glissant (1997), the pre-eminent relational island thinker, was already aware of this 'island' power for speculative thought. Indeed, he articulates this power precisely as the (logical) conclusion of the Poetics of Relation. Early in the book he draws our attention to the indifferent and withdrawn islander, who he meets in the form of a solitary man on the 'Black Beach':

This is where I first saw a ghostly young man go by; his tireless wandering traced a frontier between land and water as invisible as floodtide at night. I'm not sure what he was called, because he no longer answered to any given name ... He refused to speak and no longer admitted the possibility of language ... (Glissant, 1997: 122)

Glissant (1997: 122) says that '[i]t doesn't feel right to have to represent someone so rigorously adrift, so I won't try to describe him.' But even so, Glissant does, and he reads the man's physical gestures (without any accompanying words) as saying the following:

'I understand what you are attempting to undertake. You are trying to find out why I walk like this - not-here. I accept your trying. But look around and see if it's worth explaining. Are you, yourself, worth my explaining this to you? So, let's leave it at that. We have gone as far as we can together.' I was inordinately proud to have gotten this answer (Glissant, 1997: 123)

For Glissant, this withdrawness is a response to what he calls the Chaos of the opening up of Relation on a global scale, and the associated forces of capitalism, colonialism and other forms of oppression manifest on the Caribbean islands. It is also his response to the generosity of those who seek to save islanders, or approach them with a duty of care in their relational entanglements. Indeed, 
the book returns to this man - walking the beach, in his opaqueness and withdrawn attitude - to provide its closing lesson:

... his withdrawal [is] absolute .... As for those who follow him, if we can put it that way, (but we do know the rhythm of his passages; we are able to anticipate them), we are beginning to accept the fact that he is more resistant than we and more lasting than our endless palaver. No one could be content with this enclosed errantry, this circular nomadism - but one with no goal or end or recommencing. (Glissant, 1997: 208)

In the world of modernity, dominated by the oppressive and extractive forces of coloniality, we could perhaps read two paths of critique and resistance: one, the radical alternative telos of a newer or better form of caring modernity; the other, a much more 'minor', radical and thorough-going rejection of the imaginary of modernity itself. It is from these roots, highly productive of Caribbean subjectivities among others, that approaches of Storiation derive much of their power today. This resistance is the refusal to be captured or represented: Glissant's islander is read as defiant of power, disruptive of claims to knowledge, and resistant precisely because of this power of withdrawal. ${ }^{19}$

\section{Conclusion}

In our examination of the onto-epistemology of Storiation we have considered a number of approaches, drawn from working with islands, in speculative materialist and quantum ways, seeking to escape the modernist episteme by giving islands and islanders the power to trump the constraints and limitations of modernist thought. Here, 'becoming island' implies an openness to being influenced by the world rather than imposing the imaginaries of modernist-centred knowledge and control. Islands, as we have highlighted throughout this book, can play this role, as an alternative pole of thought, because of their imagined exclusion from the homogenising control of mainland or modernist power. The limi- 
nal positionality of the island, as part of the modernist world but at the same time seen as the preserve of non-modern relational dependencies, has enabled the island to become a significant figure for Anthropocene thought.

Our contemporary moment is very much shaped by struggle to escape from modernist constraints. This book has taken seriously the role of the island in providing the resources for work that seeks precisely this. Becoming island is not an aspiration for modernist political fighters or leaders with programmes and new agendas to impose: it is an opening to new experimental practices, ways of being and knowing that seek to 'hold' the world rather than climbing above it. This holding of the world is not a romantic gesturing, imagining the world as a harmonious and wonderous sublime; there can be no romanticising the Anthropocene. For Colebrook (2016: 124), the notion of what she calls the 'geological sublime' foregrounds how we can hold the world without recentring the subject:

The geological sublime is therefore the challenge of looking at the entire archive of the earth - including human script - as one might look at the marks left on buildings by the forces of weathering. How would we read ourselves if we were not to assume some ultimate readability or spirit beneath the materiality of text?

Storiation, as the key 'holding' analytic in our heuristic framing, seeks to 'de-theorise' the world; to start from the world rather than from the subject. It seeks to slow, to hold, thought within the world. This is a speculative practice of thinking through and with other entities rather than rushing to appropriate them. What would the world look like, how could it be known without the rush to reduce, to assimilate, to appropriate, to extract, to instrumentalise? How can we hold the world before making all too modern' cuts and distinctions? We have seen how Storiation as an onto-epistemological approach has attempted to do this in two ways. Firstly, in the refusal of modernist imaginaries of time and space, bringing what was considered 'past' or 'away' back to inform the present; to hold the occluded and excluded, the afterlives of the extractive and oppressive becoming of the Anthropocene itself. 
Secondly, in the refusal of modernist cuts of thought and matter, of subject and object; to hold these together in tension through the speculative process of decentring the thinking subject.

Storiation thereby is a practice of working with the world after the end of modernity - and in this sense is no different in its stated aspirations than the analytics already discussed, of Resilience, Patchworks and Correlation. However, the stakes of what it means to work with the world are very different in each of these approaches. The question of what it means to think and work beyond modernist constraints is the overarching problematic of the Anthropocene. A problematic in which the figure of the island has played an important heuristic role, its liminal positionality enabling it to inspire and inform a wide range of work and experimentation in both thought and practice. However, to date, little serious work has been devoted to this question. As we have been clear throughout, we have not been concerned with stating how islands should be framed or understood, but rather with methodologically and analytically starting to draw out the work that islands enable across this growing and vital area of thought. We are interested in examining the question of how islands work and are (re)worked in Anthropocene thinking. We do not see our own heuristic framing as necessarily the only way of answering this question. On the contrary, as we turn to explore further in the final chapter, we hope this book might spark discussion about the development of a more expansive critical agenda for island studies in the Anthropocene.

\section{Notes}

${ }^{1}$ Contemporary art, for example, regularly engages islands as central figures for understanding how there is no 'away' in debates about the Anthropocene. For example, Laurent Gutierrez and Valérie Portefaix (2015: 225) record the artwork 'Island is Land' about the formation of a new island in the ocean 'formed by tons of plastic garbage', and as a way of registering the traces, hauntings and afterlives of mainland modernity. As we examine in this chapter (see also, Chandler and Pugh, forthcoming a), the Great Pacific Garbage Patch is perhaps the emblematic island figure, which demonstrates how, contra 
modern frameworks of reasoning, there is spatially and temporally no outside, no 'over' or 'past'. (see also Alaimo, 2016; Somerville, 2017). Such Storiations of islands in the Anthropocene thus hold together that which modernity seeks to cut and to separate, disrupting assumptions of segmented time and space. Elsewhere, Gutierrez and Portefaix (2015: 226) record the exhibition Desert Islands, where:

the surface of the sea is translated to a fractured surface of 100 mirrors. Each mirror frames one island, as well as its geographical position to the others. Assembled into a new world map, the meticulous selection of 100 islands presents a multifaceted laboratory of major human actions and experimentations on islands - utopian communities, fiscal paradises, military spheres, clandestine migration zones, drug exchange points, prostitution hubs, and exclusive leisure areas. The 100 islands serve as a new reference, to not only feed the desires but also the fears and secrets of our time.

${ }^{2}$ In Chapter 2, we examined how the term 'Anthropocene Island' has been put to use for the development of more Resilience-oriented analytics (ecoLogicStudio, 2017b). But there are other ways in which the term has gained purchase which are more aligned with those of Storiation. Peggy Cyphers, for example, curated the exhibition 'Anthropocene Island: Colonization, Native Species and Invaders' (Pratt Institute, New York, 16-27 September 2019), with the purpose of engaging:

the vast environmental and geopolitical forces re-ordering the world as we have known it. Through the traceable singularity that is plastic (the geologic place-marker of the Anthropocene) to native and 'invasive' species, the re-worlding of migratory creatures, including humans, are examined. As a universal material of contemporary global culture, plastic endures in the environment such that all plastic ever created still exists. The petrochemical industry that fuels the relentless production of plastics is the same modus operandi that is also causing desperate attempts to extract the last drops of oil from the planet, which in turn is cooking up the enormous climatic changes we experience across the globe. Climate change is pushing all creatures - human, plant, animal and mineral - into new geolocations. The artists of 'Anthropocene 
Island: Colonization, Native Species and Invaders' examine these interconnected linkages through sculpture, drawing, photography, video and installation. (Cyphers, 2019)

${ }^{3}$ Similarly, for Cole et al (2016: 211), focusing upon the islands of Japan, 'the 2011 Fukushima-Daiichi disaster in the time of the Anthropocene' can also be understood 'as a moment when life escapes formations of categorical or territorial capture'.

${ }^{4}$ Denise Ferreira da Silva (2016: 65, emphasis in original) argues that, 'Without separability, knowing and thinking can no longer be reduced to determinacy in the Cartesian distinction of mind/body (in which the latter has the power of determination) or the Kantian formal reduction of knowing to a kind of efficient causality. Without separability, sequentiality (Hegel's onto-epistemological pillar) can no longer account for the many ways in which humans exist in the world, because self-determination has a very limited region (spacetime) for its operation.' After the end of the world of modern reasoning, turning away from cuts and separations, 'sociality becomes neither the cause nor the effect of relations involving separate existants, but the uncertain condition under which everything that exists is a singular expression of each and every actual-virtual other existant.'

${ }^{5}$ Morton (2013: 28) is fond of employing analogies in order to heighten the affective sensation of the weirdly disorienting forces of the Anthropocene; in particular regularly comparing global warming to 'the Force' in Star Wars, where he says that global warming 'surrounds me and penetrates me' but, in its totality, remains a mystery (see also Morton, 2013: 80, 85, 141). Like Luke Skywalker, sensing the Force while sitting on his island retreat in The Last Jedi, for Morton, global warming is similarly sensed in the trees, oceans, rocks, surfaces and surrounding oceans of an island. But there is always an essentially ungraspable mystery, the humbling ontological 'rift' between the vast multidimensions of global warming and humans sitting on an island, who are unable to read them in their totality (Morton, 2013: 18). There is no escape, no isolated or insulated island. As avid fans of Star Wars trivia will know, the island which Luke Skywalker spends his time in self-imposed exile on, in The Last Jedi, is actually a real island called Skellig Michael (off Ireland). As Gillis (2004) writes, Skellig Michael had a particularly important role in medieval European Christianity; which, influenced by the idea that Christ encourages exile from the material world, understood isolated islands, such as Skellig Michael, as the holiest sites in 
their world for such escape and purity. These were 'closely associated with the supernatural, with extraordinary events, heroic adventures, and divine revelations, with mythical beginnings and endings' (Gillis, 2004: 37). Thus, it is important to note that, in Morton's work, islands play a very different ontological and spiritual role. For Morton, if the great mystery of hyperobjects, like global warming, is withdrawn, then we can only look for signs, or signals, or effects; never know reality itself. We can only speculate upon how each ant, blade of grass, or person walking on the island experiences the Anthropocene.

${ }^{6}$ Nigel Clark (n.d.) examines the artwork of Melinda Rackham's a.land, which similarly works with an 'island imaginary' to convey a 'succession of porthole perspectives - a kind of channel switching through diverse domains - suggest[ing] that the totality of the world is beyond our grasp ... This is a world where form is always provisional and transmutation a constant possibility.

${ }^{7}$ Our access to the world is always indirect or mediated (the same for all other entities); thus, while there is only one reality there are necessarily many 'worlds' formed through infinitely differently forms of mediation, which are always partial and contingent. We have access to data from which we can infer causation or correlation, but this data is neither the reality of the object, of the instruments or of the subject itself. If we act as if there is only one 'world', and that this is a world that we have access to, we anthropocentrically see just what appears to us - for example, if we see that washing hands kills bacteria $(p)$ then we don't see that washing hands enables bacteria to live, to change and develop resistances (not-p). Morton (2016b: 65, emphasis in original) argues, therefore 'We are going to have to think things as weird. What is required is that we break out of Aristotle's Law of Noncontradiction where 'You can't say $p$ and not- $p$ at the very same time' (Morton, 2016b: 74). Life needs to be seen as contradictory, weird or strange to enable us to think more openly, ecologically and responsibly.

${ }^{8}$ Such approaches are widespread: for example, commenting upon Coronavirus, Alex De Waal (2020) says: 'Perhaps the most difficult paradigm to shift will be to consider infectious agents not as aliens but as part of us-our DNA, microbiomes, and the ecologies that we are transforming in the Anthropocene.

${ }^{9}$ For Alaimo (2010: 158), Darwin's work on islands and evolution 'casts the human within an evolutionary narrative in which we are not immune from the forces of messy, unpredictable materiality ... a 
material world that is never merely an external place but always the very substance of our selves and others.'

${ }^{10}$ As Morton (2017: 282) says, 'Of all people, it was Charles Darwin who opened the gate to the spectral world ... When one collapses the life-nonlife boundary and relaxes the human-nonhuman boundary, all kinds of spectral creatures start to be seen, nightmarish beings that scuttle about. That are not categorizable. Yet they exist. They look like nightmarish beings because of the extreme pressure they exert on existing frames of reference, existing categorical boxes. ... But when the boxes dissolve, are these beings intrinsically horrifying? Is the gothic view of these beings the only view, for the rest of time, or is it a temporary effect of the pressure that such beings place on categories such as life and nonlife?'

${ }^{11}$ As another example, Melody Jue's (2020: 73) Wild Blue Media: Thinking Through Seawater draws upon the writing of Vilém Flusser and turns to the 'vampire squid' and the oceanic abyss:

as a speculative environment, constructing a 'molluscane point of view' to imagine how an intelligent aquatic organism would develop different concepts to orient itself to its world than those familiar to the dry landscapes of human thought. The abyss dramaticizes the ocean's condition of ephemerality, where inscription on paper or even stone tablets would eventually be eroded by seawater or encrusted with growth. Because the vampire squid lives in a milieu where not all our vocabulary or figurative language works smoothly, it pushes us to consider more ocean-specific conditions of mediation and a vocabulary that would be adequate to the abyssal environment. (Jue, 2020: 73)

12 Thus, for the onto-epistemology of Storiation: 'evaluative questions are thoroughly pragmatic, but with a speculative edge. They bear on how practice speculates on what it can do, in the very form of its unfolding modal mix of activity, as it is borne witness to by the manner of effects it produces - and through which it is witnessed selfproducing as event-medium, flush with the immediacy of the occurrent emergence that it composes and that composes it ... Immanence: many lives (in expressive potential)' (Massumi, 2019: 183).

${ }^{13}$ In other work, DeLoughrey and Flores (2020) focus upon the Caribbean submarine and island shorelines as important ways of tracing and holding together the legacies of colonialism and modernity. They quote Alaimo (2011: 283): 'Submersing ourselves, descending rather 
than transcending, is essential lest our tendencies toward Human exceptionalism prevent us from recognizing that, like our hermaphroditic, aquatic evolutionary ancestor, we dwell within and as part of a dynamic, intra-active, emergent, material world that demands new forms of ethical thought and practice.' (see also Neimanis, 2018; Peters and Steinberg, 2019; Barker, 2019). For DeLoughrey and Flores, the artworks of Tony Capellán, Jean-Ulrick Désert, María Magdalena Campos-Pons, Nadia Huggins, and David Gumbs are illustrative of such an approach. They engage with how Capellán's 'sculptural work almost exclusively originated with objects, mostly plastic, that washed ashore in Santo Domingo, Dominican Republic' (DeLoughrey and Flores, 2020:312). Thus, the island is not coherently separated from the oceanic or the submarine and 'Immersion in this piece becomes invasion, an excess of the waste of capitalist consumption that challenges any notion of the sea as wilderness or space of pure nature' (DeLoughrey and Flores, 2020: 134; see also Arnall and Kothari, 2020). In another stark example, Canadian artist Kelly Jazvac turned to 'plastiglomerate', found on a Hawaiian beach in 2006 by oceanographer Charles Moore, to illustrate 'how the Anthropocene era is leading to the formation of new man-made minerals' (Yalcinkaya, 2019). This 'hybrid material is the result of plastic items washed up on the shore fusing with shells, sand and other natural materials when burnt in campfires lit on the beach' (Yalcinkaya, 2019).

${ }^{14}$ There are slightly different circulations of this passage. In a text, Brathwaite (2007: 158) says the 'sea was slake grey of what was left .... See also Christina Sharpe's (2020) reading of Brathwaite's Dream Haiti. These subtle differences are not our focus, however, as they do not change the point being made.

15 Yountae (2016: 120) writes that colonialism in Caribbean islands is a 'haunting historical wound,' that must be understood intrarelationally as the 'primary material weaving [their] physical texture' Similarly, for DeLoughrey (2010: 703), the Atlantic Ocean is 'a place where the haunting of the past overtakes the present subject'; something which, as Karen Salt (2017: 61) has remarked, 'places haunting back into the environment, and into materiality, or matter'.

${ }^{16}$ This aligns well with da Silva's (2017) bringing together of race and colonialism in what she calls 'fractal thinking', which is 'a composition, or decomposition depending upon the possibility to interrupt the unfolding of a discourse - philosophical, historical, sociological, anthropological, etc. - which would otherwise allow for a presentation 
of a situation that always justifies violence ... '. In da Silva's approach, as with Sharpe and Moten, the 'outside' of an unfolding discourse is held together in superposition, and 'the composition in the fractal is the positioning of those different moments in time and space, but as part of the same context' (da Silva, 2017). Such fractal thinking about race and colonialism acts as a 'holding' space for speculative possibilities: 'My interest in the thing is not an interest in its essence but is an interest in what else about the world could be known if modern knowledge had not limited itself as much as it did' (da Silva, 2017).

${ }^{17}$ It is, of course, also possible for a Correlational approach to register the legacies and afterlives of colonialism if they display a constant relation over time. For example, Jessica Johnson's (2019) discussion of the project 'Xroads Praxis', which employs satellite imagery to reveal how the lights went out after Hurricane Maria hit Puerto Rico in 2017. For Johnson (2019), 'Xroads Praxis' is a way of sensing the interweaving relationship between transforming environmental conditions and the legacies of colonialism on islands; what she calls a black diasporic technology for exploring what digital and analog landscapes hide and reveal':

... blackness in the blackened spaces hit hardest by Hurricane María disappeared in the aftermath. In Carolina and Loíza, towns in the constellation near San Juan, that some one-fifth to three-quarters of the population (respectively) described itself as of African descent went without mention in the aftermath of the storm. In Poncé, which did not restore electricity to all its residents until August 2018 - 328 days after the storm - between 10 and 20 per cent described themselves as black. Indigenous spaces suffered similar disappearances. Utuado, which for generations claimed indigenous patrimony on the island and is the home of Caguana Indigenous Ceremonial Park, barely rated a mention in mainstream news generated by the storm. Nor does Utuado, a region where even before the storm running water and electricity were privileges not rights, appear in the satellite image. (Johnson, 2019)

${ }^{18}$ To take another illustration of the onto-epistemology of Storiation which foregrounds speculative practice, Neimanis (2019: 504, emphasis in original) has examined oceans and wrecks as sites and holding spaces for dwelling in the legacies, hauntings and dissolve of modernity, and for the associated importance of speculative thought: 
Sea level rise, of course, is not a metaphor. Neither is the melting ice that has given up on its terrestrial existence, nor the very real thermal expansion of the oceans that is evermore inundated with our panting hot breath, to take from the sea her own ... We may not know what comes next. We are crossing. There is something that exists in the caesura, in the pause between inhale and exhale that is also called aspiration, between feet firmly planted in the sand and weathers that flow outwards, downwards, and maybe upwards again in these naturalcultural meteorological cycles. Here, there is something we still do not know ... Everything is now. Maybe the diver will become whale, who gave up on terrestrial life three million years ago, returning to the deep as five fingers of a hand were slowly covered over by a flipper. Maybe all of a sudden we will find our feet are floating. Maybe, like whales as the seas rose around them, we will learn to notice by echolocation - a skill in which knowledge is utterly dependent on learning to listen differently. Maybe we begin our dissolution by breathing, and learning, and listening; by helping to foster practices of fugitivity and care ... A singular life, a racialised structural lifeworld, the microclimate of one species, the whole ocean: who is to say what matters more, or less? In any case, the Anthropocene will demand that we become other than ourselves, and at least other-than-the human as we know it. If this is to be mourned, it is also surely to be welcomed.

19 There is a historical tradition which documents this trope, most famously, when Caliban says to Prospero on the island of The Tempest, 'you taught me language, and my profit on't, Is I know how to curse'. This can be taken literally as Caliban saying that he is reduced to frustrated cursing because the (colonial) language he has been taught by Prospero is not up to the task of connecting Caliban to his island (see Pugh, 2013). This enables a reading of the final scene between Caliban and Prospero, as a demonstration of the weakness and inadequacy of colonial language when it comes to grasping the islands and the islanders of the New World. Thus, as Shakespeare (2002) acknowledged, even as they were being 'discovered' by Europeans, during his time, islands and islanders were already doing disruptive, liminal, 'work' as withdrawn spaces; the ungraspable 'outsides' of mainland modernity. 


\section{Conclusion: A Critical Agenda for the Anthropocene}

\section{Introduction}

Islands have become one of the most emblematic figures of the Anthropocene. In responding to the lack of consideration of why islands have come to the fore in analytic approaches associated with the Anthropocene we wanted to move beyond islands as merely becoming endangered or threatened - symbolising the impacts of global warming, nuclear fallout, colonialism, rising sea levels, the displacement of peoples, intensified hurricanes, coral reef degradation and other forces associated with planetary changes. We wanted to do more than write about islands in the Anthropocene and to instead examine how islands have themselves been productive of our understanding of the Anthropocene condition. A condition in which it is understood that our modernist assumptions of scientific progress and capacities to know and shape our external world have been fundamentally questioned by climate change and environmental unpredictability.

Our project has been concerned with examining how major themes of Anthropocene thinking engage islands and islanders, and

\section{How to cite this book chapter:}

Pugh, J. and Chandler, D. 2021. Anthropocene Islands: Entangled Worlds.

Pp. 179-192. London: Ubiquity Press. DOI: https://doi.org/10.16997

/book52.f. License: CC-BY 4.0 
work with island imaginaries, in the development of methods and approaches to the world that move us beyond the modernist episteme. As we have argued, the liminal positionality of islands within modernity - as part of the world but excluded from linear and universalist imaginaries of progress and civilisation - gave the island powers and affordances which have come to play a significant role today. Islands are often worked with and drawn upon to illustrate the world in real and vital ways, which go beyond the constraints of a modernist imaginary. Islands have thus come to symbolise strengths and capacities that modernist abstract and reductionist understandings cannot grasp. Islands have become saving figures in the Anthropocene. Saving connections, dependencies, knowledge practices and relations that have been lost in modernity and now need recovering.

The Anthropocene has put the island to work and works with islands in what we think are often fascinating and spectacular ways. Never has there been a more exciting time to be an island scholar. After the end of the world of modernity, after the end of the taken-for-grantedness of the modernist assumptions underpinning Western social and natural sciences, islands are helping to reconstitute possibilities of other worlds. Thus this book has focused upon how Anthropocene thinking works with and engages islands and island imaginaries in the development of non-modernist ontologies and onto-epistemologies; widely held as key to thinking beyond the limits of the modernist, mainland, world. In the following section of this chapter we provide a general framework which allows us to reach the main conclusions of this book. In the closing section, we seek to sketch out a critical agenda for island studies and some of the key questions and issues at stake.

\section{After the End of the World, the Age of Islands}

In this section we situate this book's analysis of islands as key sites of relational entanglements, awareness and feedbacks within a broader paradigm shift which is presently taking place in 
contemporary thinking associated with the desire to move critical thought beyond the limits of the modern episteme. As the Caribbean island scholar Sylvia Wynter (2003) observed, leading Western traditions of social and political thought have projected power and rationalised coloniality through imposing the hierarchical idea of a human/nature divide, overrepresenting 'Man' at the centre of the world. Today, the hold of the human-centred or modernist episteme is being questioned, no more so than in relation to the Anthropocene. Today, attempts to go beyond the constraints of Western, Eurocentric or modernist conceptions of the world are oriented towards questions of relational entanglements, awareness and feedbacks. Islands have thereby moved from the periphery to become more important in broader contemporary thought, precisely because they are widely understood to be productive for these alternative approaches. In heuristically presenting the analytics of Resilience, Patchworks, Correlation and Storiation, we reach conclusions concerning how and why drawing upon islands works to enable and create new possibilities for thought, generative for Anthropocene thinking.

As we have discussed in the previous chapters, the need to think beyond the modernist human/nature divide is perhaps the key driver of Anthropocene thinking. Novel and alternative approaches to modern reasoning, such as the ontologies and ontoepistemologies we have discussed in this book, develop from this starting point. Here Wynter (2015) stands out as a scholar who has devoted much of her career to explaining how dominant theoretical frameworks of Western or 'mainland' thought have long grappled with the problem of 'Man' as separated from 'nature'. Indeed, for Wynter, one way in which this problem was addressed was through the construction of 'man' as a 'natural organism', like and also unlike any other. She highlights that it was Darwin, writing in the 1800s, who sealed the overturning of the Latin-Christian tradition, initially questioned by Copernicus a few centuries earlier, where 'man' was posited as separate from or above nature. This is why, as we have noted, contemporary authors such as Stacy 
Alaimo (2010; 2016), Cary Wolfe (2017) and Timothy Morton (2017) call Darwin the first posthuman thinker.

What Wynter (2015) calls 'Man 2' is the subject of a particularly powerful story - what she calls, following Frantz Fanon, a 'mythoi' - based on the 'premise that the human is ... defined biocentrically and therefore exists, as such, in a relationship of pure continuity with all other living beings (rather than in one of both continuity and discontinuity)' (Wynter, 2015: 16-17). Thus 'Man 2' historically differentiates itself through a 'normally, imperatively self-correcting ... order of knowledge' (Wynter, 2015: 16 , emphasis in original). Man 2 is not an exception, existing collectively as a species distinct or apart from nature, but at the apex of a purely biological framing of being, understood to be selfadaptive and self-correcting:

This is the version in whose terms the human has now been redefined, since the nineteenth century, on the natural scientific model of a natural organism. This is a model that supposedly preexists - rather than coexists with - all the models of other human societies and their religions/ cultures. That is, all human societies have their ostensibly natural scientific organic basis, with their religions/cultures being merely superstructural. All the peoples of the world, whatever their religions/cultures, are drawn into the homogenizing global structures that are based on the-modelof-a-natural-organism world-systemic order. (Wynter, 2015: 21, emphasis in original $)^{1}$

Anthropocene analytics can be read as building upon but also moving beyond Wynter's historical understanding (particularly the analytics of Patchworks and Storiation, furthest from the modernist framework). Most Anthropocene thinking today accepts that humans are not separate from the world. Indeed, the overarching problematic of Anthropocene thinking is that of relational entanglement, from which new questions, approaches and analytics, such as those examined in this book, emerge. As Eva Giraud states (2019: 1), today the consensus seems to be that 'the human is only realised by and through its relations with other 
entities ... [and that this is widely seen as offering] a source of ethical and political potential.' Likewise, Claire Colebrook and Jami Weinstein (2017: xxi) argue that '[o]nly once the problem has yielded a certain stability can questions emerge.'2 Thus, in different ways, the four analytical framings which we have presented here are illustrative of how working with islands as figures of relational entanglement drives thought beyond modernist, human-centred, constraints. This is why we believe the liminal figure of the island has become important as a resource to work with for drawing out relational approaches to being (ontologies) and knowing (ontoepistemologies) in the Anthropocene.

In this book, we have presented relational ontology as a continuum, as legacies of modernist assumptions are peeled away. Some approaches, such as Resilience, more straightforwardly build upon and work with the metanarrative of Wynter's Darwinian Man 2 who adapts to transforming planetary conditions (even if today it is the Indigenous islander who is often heralded as more adaptive or resilient than the mainland Westerner or European). As we examined (in Chapter 2), Resilience is the art of adaptive change in relation to changing circumstances. Here, drawing upon certain imaginaries of island life is understood to be particularly productive for Resilience thinking; because islands are held to exemplify the powers of creative and productive differentiation and individuation. In contrast to the homogenising, modern notion of a human/nature divide, islands are seen to exemplify the powers of immanent, inter-dependent life which - as Darwin worked to reveal through his influential research on islands works in more adaptive and dynamic ways.

In Chapter 3 we turned to the more fluid relational ontology of what we called Patchworks. This both develops and disrupts the ontology of Resilience thinking. In Patchworks, the Resilience imaginary of islands existing in a flat, two-dimensional space, side-by-side, is replaced with a more open island ontology of spatial and temporal becoming. In Patchworks, the world dissipates into patchworks of novel and often partial interconnections. This destabilises the 'solutionist' or instrumentalising aspects of 
Resilience, making Patchwork approaches less governmentalising and human-centred. Patchworks is about opening ourselves to the relational affects and knots of co-relational entanglements. We make, explore and journey in Patchwork ontologies, rather than merely reflecting upon and becoming more aware of our relational interconnections so as to become resilient. Here we explored how island ontology becomes the ontology of the world and thinking with islands becomes a 'verb' and a practice of 'world-making' (Teaiwa, 2007: 514). Thus, Patchwork ontologies align with broader trends in Anthropocene thinking which emphasise the importance of 'staying with the trouble' (Haraway, 2016), as life becomes less predictable, confineable and graspable. The focus is upon 'giving-on-and-with' (Glissant, 1997: 142) the power of disturbances and emergent effects, where island ontology becomes a key resource for stimulating thinking about how relationality is radically open and contains potentialities or possibilities which are beyond our capacities to predict or to control.

After examining these relational ontologies, we turned to how islands have been put to work in rethinking how we know after the world of modernity. Again, presenting an onto-epistemological continuum as the reality of the world was conjured to enable a move beyond the Kantian prison of representation. Two productive approaches to onto-epistemology were put in relation, both crucial to informing and generating thought in the Anthropocene; these we labelled as Correlation and Storiation. Both also centre upon the importance of relational entanglements and affordances as a way of generating knowledge about the Anthropocene, and both therefore often also engage islands as important sites for generating such understanding. Where they differ is in how they register or 'read' relational entanglements and affordances. Correlational analytics focus upon how inter-relations or 'actants' have particular capacities or affordances which enable human knowledge of changing environmental conditions. Entities do not therefore have a core essence or meaning in themselves, as they do in modern frameworks of reasoning. Rather, knowledge is established inter-relationally by undertaking correlational practices 
through discovering how communicative interaction organically worlds the living world and thinking through how this can be replicated through high-tech interventions, such as Big Data and the Internet of Things. Here, drawing upon and engaging island life has been significant for the development and proliferation of Correlational approaches in Anthropocene thinking, as islands are widely held to be the emblematic correlational registers - the 'canary in the coalmine' of climate change - enabling humans to materially register otherwise unseen planetary forces.

At the other end of the onto-epistemological continuum, we examined how Storiation draws upon islands as sites of relational entanglement in ways that enable relations to sustain or hold the world beyond representational understandings of fixed grids of time and space. Here we examined how the notion of intra-action, rather than inter-action, captures this shift; registering and holding together that which modernity tears apart. This disrupting of linear framings of space and time and of separations between subjects and objects has been put to a range of uses. Work in this area has been important in opening up new possibilities for rethinking colonial legacies and environmental side-effects through understanding that care and accountability extend the present into both the past and future. The afterlives and ongoing effects of colonialism are still with us as much as our actions today will reverberate through the ecosystems of the future in ways which stretch beyond our capacities to calculate or to imagine. Holding temporal, spatial and agential divides together, this onto-epistemology rejects the notion that humans can distance themselves from the ongoing effects of such forces as colonialism, global warming, nuclear radiation and waste production which unfold in time and space in 'weird', 'quantum' or 'haunting' ways.

In different ways then, Anthropocene analytics are based on the premise that humanity is living after the end of the world (that is, after the modernist construction of the world on the basis of the human/nature divide, which structured scientific, social and political thought). The problem of the human/nature divide has been 'resolved' and moved beyond via approaches which focus 
upon relational entanglement, feedbacks and surprising connections. From this open space of possibility, new questions have emerged, driving debate forward, and engendering new approaches. Throughout this book, we have sought to document this schematic shift through a close examination of how islands have emerged as increasingly important sites in Anthropocene thinking. Together, as authors, we have been interested in pursuing how the figure of the island has become understood to be so productive and generative for challenging and moving beyond the constraints of modernist forms of thought.

One of our main contentions is islands and island cultures are seen to be highly useful or generative for such debates precisely because islands and the islander have long been imagined as liminal figures, marginalised as lacking key rationalist attributes of modernity and labouring under relational ties and dependencies. A minor tradition that sought to utilise and to value these capacities and dependencies that modernity sought to reject - from Darwin to Strathern, Glissant and Brathwaite - thus was already available as a resource to be drawn upon. Whilst, as we have explored in the preceding chapters, these approaches are of course different from each other, they nevertheless all seek to move beyond and to challenge the key assumptions of modernist thought, opening up ways of thinking that do not assume that there is a separate human subject (disentangled from the world) or a world (as a coherent object of knowledge). In these ways it could be argued that the rise to prominence or the centring of islands to contemporary thought is in many ways overdetermined. It had to be. As Derrida (2011) astutely brought to our attention many years ago, once we finally realise the end of the 'world' as a coherent concept, we come to realise that there are only islands. Contemporary debate is really a coda or a footnote to this insight: What sort of islands? What is at stake in a world of island-becoming or becomingisland? Anthropocene thinking is essentially a question of what it might mean to work and think with islands and island imaginaries. The liminal figure of the island appears to assert its power and authority upon the Anthropocene as a new world of relational 
entanglements, feedbacks and weird reworkings of relations across time and space.

\section{The Power of Thinking with Islands: A Critical Agenda for Island Studies in the Anthropocene}

We now turn to what a critical agenda for island studies in the Anthropocene might look like going forward. The purpose of this would be to expand the analysis developed in this book of why and how islands are being drawn upon in Anthropocene thinking. Whilst we hope that readers will see this book as a useful starting point for sparking discussion, there will no doubt be many other ways of working through this question as well; not all of which will necessarily be associated with the problematic of relational entanglements, feedbacks, or the broader shift towards speculative forms of thought, which we have analysed across the previous chapters. These are just the main reasons why we think Anthropocene thinking draws so heavily upon islands and island imaginaries. We are keen to hear about others. We want to encourage an open-ended, convivial approach, asking readers to consider our overarching argument and four heuristics, but to also reflect upon and suggest other analytics or approaches which may be applied to understand why and how working with and upon the figure of the island is so generative for Anthropocene scholarship and related practices.

At their best, island studies are of course always critically and productively reflective of how the figure of the island has been written about and worked with throughout history. So why insert the word 'critical' at this current juncture and, specifically, with regards to debates about the Anthropocene? Is this really necessary? We decided that, given the generative role of islands and the variety of ways in which they are (re)worked in key Anthropocene discussions, using the term critical, for us, is about injecting a certain sense of urgency into these debates. Whilst the term 'critical' might immediately imply to some readers some reworking or extension of Western critical theory, for us it does not. A critical 
agenda for islands studies in the Anthropocene is about mapping how Anthropocene thinking draws upon and thinks with islands, however and wherever this is taking place. For example, in this book we have focused upon the intersections between Anthropocene scholarship, essentially in the Western academy, and island scholarship more broadly, but it would also be extremely useful to examine how writers, artists and activists from other spaces and locations - such as mainland China or India - engage with islands within the contexts of debates about transformative planetary changes as well.

Without being prescriptive of how such a critical agenda could unfold, we do however believe that any such agenda will need to keep one central concern or operating logic in mind: if we are to examine the power or force of islands in Anthropocene thinking (that is, why and how islands ingress so deeply and productively), then we cannot separate out island imaginaries and broader trends in social and political thought from the material characteristics of islands as geographical forms which are doing important 'work' in such debates. For research and scholarship to more completely understand how and why work with islands has become generative, there is a need to orient around a purposefully interdisciplinary research agenda that engages the material and physical world as existing simultaneously with island imaginaries and contemporary developments in social and political thought. It is only by taking this point seriously - that there is something about islands (existing simultaneously in material form and thought) - that we can examine how and why they enter into and are put to work in Anthropocene thinking. ${ }^{3}$

There are many different ways of getting this key point across. For us, Alfred North Whitehead and Frantz Fanon do so particularly effectively, but readers will no doubt be aware of many other ways to focus upon the importance of developing situated knowledges which do not separate out the material world from how it is thought. As Whitehead $(1985,1968,1967)$ said, thought should be understood as in the world, rather than as about the world. This is a profound statement. The subject and thought itself are 
neither primary (as a Kantian starting point) nor secondary (as in the privileging of ontology) but are always already in the process of world-making. The Anthropocene or islands do not exist 'out there' or 'in our heads'; rather, ontological statements made from working with them - such as the relational ontologies or ontoepistemologies examined in this book - should be understood as objective facets of the given world itself (existing simultaneously in materiality and in thought). For Whitehead (1985: 4), '[o]ur datum is the actual world, including ourselves; and this actual world spreads itself for observation in the guise of the topic of our immediate experience.'

Thus, how islands are thought, how and why they appear and the powers they have in Anthropocene thinking, tells us simultaneously about islands, the Anthropocene, ourselves, and our own shifting preoccupations. For Whitehead, there could be simply no separation; how humans think about islands and the Anthropocene is simultaneously both product of the world and its producer. It is this fact that permits us to conclude, from our own research presented here, that Anthropocene thinking draws upon islands because the Anthropocene and islands both work to foreground a world of relational entanglements (in the materiality of the world and in thought). The dominance of this particular problematic means that only certain questions get raised, rather than others, and it is why certain analytics, particular geographical forms like islands, relational ontologies and onto-epistemologies, such as the ones examined in this book, are understood to be more generative for current debates.

Here we therefore agree with Isabelle Stengers' (2008; 2014) approach when she says that Whitehead's crucial insight enables a move beyond merely dismissing or destabilising the grounds for truth claims; instead, productively stressing the importance of taking these claims seriously for the development of thought in the world: 'Whatever we call a cause, even a physical interaction, has no power to cause independently of the way in which it will be grasped in a subjective process of self-production' (Stengers, 2008: 103). For us, then, what is central for any critical research agenda 
is not that some objective truth about islands or the Anthropocene is revealed, but how the claims which are made (and the way in which they are presented) speak to us about our contemporary Anthropocene condition (see also Chandler and Pugh, forthcoming a; forthcoming b). This is why we take a particular interest in schematic and analytical shifts in thought as taking place in the world, such as those presented in this book. The emphasis is upon how our concrete experiential or worldly consciousness appropriates or receives islands and the Anthropocene.

For Whitehead, as Stengers (2008: 98) states, to underplay the importance of this would result in the 'fallacy of misplaced concreteness', where we imagine that we are merely observers reflecting, meditating or speculating upon the world, rather than being ourselves its products and producers (Whitehead, 1967). As Vicky Kirby (2011: 133) also highlights, drawing upon Karen Barad, there is no thought without intentionality, the 'desire to know, is implicated in the very ontology of what [the researcher] is looking at' (see also Stengers, 2008). This is the crucial point we take from Whitehead; one that has long featured in social sciences and humanities' concerns of positionality and the importance ascribed to situated knowledges rather than abstract understandings. Reading Whitehead in particular, for us, sharpens this need to see thought - such as thinking about islands in the Anthropocene - as an agential product of our being-in-the-world.

But perhaps Frantz Fanon (1967) enables us most effectively to get this point across about how the material world and thought exist simultaneously. As Fanon famously said, whether certain ways of thinking about being - certain ontologies or ontoepistemologies - become more alluring and influential in debates tells us what we think it means to engage with the world. Fanon (1967: 176) criticised those engaged in anti-colonial struggles who only focused upon one side of being or ontology, for retreating into a 'universal standpoint', without addressing how the world, in thought and materiality, had given rise to these understandings in the first place (see also Wynter, 2015). Thus, we can similarly say that, in the Anthropocene, what certain ontologies and 
onto-epistemologies foreground, or downgrade, not only makes us aware of new things about islands and islanders and the work they do for contemporary imaginaries. It also tells us important things about ourselves as scholars engaging the Anthropocene - about our own preoccupations and how these are changing, opening up, or limiting possibilities in new ways. As Fanon (1967) recurrently argued in The Wretched of the Earth, how we understand ontology and being is vitally important, but this understanding is also the product of work, struggle and labour; and, therefore, reveals things about ourselves, how we direct our efforts, and how we seek to frame the stakes for engagement as well.

From this we can suggest that, in addition to understanding the material world and thought as existing simultaneously, a critical agenda for island studies in the Anthropocene could ask such questions, in no particular order, as: How and why do islands become appropriated in Anthropocene thinking? How does the liminality of the island for modernist thought endow the island figure with certain powers and affordances? What makes particular aspects of islands attractive for such thinking? How do approaches cohere around certain analytics or heuristics, rather than others? What are the various modes of affect and what capacities for becoming affected are being engendered? How are relational effects understood and put to work? What makes the island more 'real' than the mainland? How does drawing upon island and islander life in Anthropocene thinking show us the world, or enable us to enter the world? How does the figure of the island enable us to think in terms of immanence as product and producer of the world? What does it mean to make claims to nonhuman or to speculative knowledge? How do certain ways of drawing upon islands and island imaginaries stabilise, detour, or become disruptive in Anthropocene thinking? How does work with islands hold contradictions in creative tension? We believe that these kinds of questions are important to ask for opening up critical possibilities for island studies in the Anthropocene.

For us then, to repeat, the question is not whether any relational ontology or onto-epistemology that we have discussed in this book is necessarily right or wrong. Rather, it is about framing a critical 
research agenda in terms of how the widespread development, appeal or lure of certain ontologies or onto-epistemologies reflects how the world and our purposes as researchers, scholars and activists are changing. Informed by Fanon's insistence that human understanding, thought and claim-making can only be understood as dynamic processes of work, struggle and labour, this is the question which concerns us most, going beyond the remit of this book. Examining why and how certain ontologies and onto-epistemologies have emerged, developed and have appeal becomes a way into the world - into understanding how the critical stakes of the Anthropocene and islands are being understood and engaged - rather than a way of abstracting and separating ourselves from them.

\section{Notes}

${ }^{1}$ For Wynter, it is the story of 'Man 2' which has enabled such forces as colonialism, racism and related oppressions, to flourish and become so deeply entrenched across the world: i.e. the myth that some humans (often understood as White, Western, male) are more exceptional than others at flourishing and adapting to environmental conditions. As Wynter (2015: 22) points out, the failures of the anti-colonial and civil rights struggles resulted in many others also buying into this 'mythoi' of Man 2: 'What other model was there?'

${ }^{2}$ In order to clarify what they mean here by the raising of a 'problem', Colebrook and Weinstein (2017: xxi) employ such everyday examples where, 'Questions - such as whether drugs should be legalized or whether there should be international intervention in human rights violations - are only possible if problems are not composed. What might it be to question the very being of drugs and the notion of the proper human body and its external supplements? What might it be to ask how it is that something like a human right could act as a weapon in international war or politics? Questions that seem to have ready answers - yes or no, pro- or anti- - are only possible because of previous problems that have now lost their tension.'

${ }^{3}$ We recently explored this agenda in Dialogues in Human Geography (see Grove, forthcoming; Wakefield, forthcoming; Sheller, forthcoming; Davis, forthcoming; Colebrook, forthcoming; Perez, forthcoming; Burgos Martínez, forthcoming; Chandler and Pugh, forthcoming $a$, forthcoming $b$ ). 


\section{References}

Adger W. N. (2000) Social and Ecological Resilience: Are they related? Progress in Human Geography, 24: 347-364.

Alaimo S. (2016) Exposed: Environmental Politics \& Pleasures in Posthuman Times. Minneapolis, MN: University of Minnesota Press.

Alaimo S. (2014) Oceanic Origins, Plastic Activism, and New Materialism at Sea. In S. Lovino and S. Oppermann (eds) Material Ecocriticism. Bloomington, IN: Indiana University Press, pp. 186-203.

Alaimo S. (2011) New Materialisms, Old Humanisms, or, Following the Submersible. NORA-Nordic Journal of Feminist and Gender Research, 19(4): 280-284.

Alaimo S. (2010) Bodily Natures: Science, Environment, and the Material Self. Indianapolis, IN: Indiana University Press.

Alexander R. (2016) Migration, Education and Employment: Sociocultural factors in shaping individual decisions and economic outcomes in Orkney and Shetland. Island Studies Journal, 11(1): 177-192.

Allar N. A. (2019) Rhizomatic Influence: The Antigenealogy of Glissant and Deleuze. Cambridge Journal of Postcolonial Literary Inquiry, 6(1): 1-13.

Alliance Magazine (2012) Interview - Buzz Holling, Alliance Magazine. December, 2012, https://www.alliancemagazine.org/feature/interview -buzz-holling 
Anderson B., Grove K., Rickards L. and Kearnes M. (2020) Slow Emergencies: Temporality and the racialized biopolitics of emergency governance. Progress in Human Geography, 44(4): 621-639.

Annan K. (1999) At a Glance: Small islands developing states. A message from Kofi A. Annan Secretary-General of the United Nations. https://web.archive.org/web/20160912151839/http://www.ourplanet .com/imgvers n/103/08_small.htm

Arnall A. and Kothari U. (2020) Becoming an Island: Making connections and places through waste mobilities. Transactions of the Institute of British Geographers. E-pub ahead of print. DOI: https://doi .org/10.1111/tran.12391

Babbage C. (1837) Exhumed and Exhaled. Lapham's Quarterly. https: //www.laphamsquarterly.org/memory/exhumed-and-exhaled

Bahn P. and Flenley J. (1992) Easter Island, Earth Island. London: Thames and Hudson.

Baldacchino G. (2020) Prologue. Geographies of Hope and Despair: Land cover and land use on islands. In S. J. Walsh, D. Riveros-Iregui, J. Arce-Nazario and P. H. Page (eds) Land Cover and Land Use Change on Islands: Social \& Ecological Threats to Sustainability, E-pub ahead of print. DOI: https://doi.org/10.1007/978-3-030-43973-6_1

Baldacchino G. (2018) Seizing History: Development and non-climate change in small island developing states. International Journal of Climate Change Strategies and Management, 10(2): 217-228.

Baldacchino G. (2008) Studying Islands: On Whose Terms? Some epistemological and methodological challenges to the pursuit of island studies. Island Studies Journal, 3(1): 37-56.

Baldacchino G. (2006) Islands, Island Studies, Island Studies Journal. Island Studies Journal, 1: 3-18.

Baldacchino G. and Clark E. (2013) Guest editorial introduction: Islanding cultural geographies. Cultural Geographies, 20(2): 129-134.

Baldacchino G. and Royle S. A. (2010) Postcolonialism and Islands: Introduction. Space and Culture, 13: 140-143.

Barad K. (2019) After the End of the World: Entangled nuclear colonialisms, matters of force, and the material force of justice, Theory \& Event, 22(3): 524-550.

Barker J. (2019) Confluence: Water as an analytic of Indigenous feminisms. American Indian Culture and Research Journal, 43(3): 1-40.

Barnett H. (2017) Working with Physarum Polycephalum and EcoLogicStudio, Resilient Topographies \#1: the peninsula of Paljassaare, https: //www.youtube.com/watch?v=qRzRXUTkgP0. 
Barnett J. (2001) Adapting to Climate Change in Pacific Island Countries: The problem of uncertainty. World Development, 29(6): 977-993.

Bateson G. (2000) Steps to an Ecology of Mind. Chicago, IL: University of Chicago Press.

Beer G. (1997) The Making of a Cliché: 'No man is an island'. European Journal of English Studies, 1(1): 33-47.

Benítez-Rojo A. (1996) The Repeating Island: The Caribbean and the Postmodern Perspective. Durham, NC: Duke University Press.

Bennett J. (2010) Vibrant Matter: A Political Ecology of Things. Durham, NC: Duke University Press.

Benwell R. (2011) The Canaries in the Coalmine: Small states as climate change champions. The Round Table, 100(413): 199-211.

Berman R. (2017) Moose may be the Real Canaries in the Coal Mine: A study of moose skull sizes on a remote island shows the effects of global warming. Big Think. https://bigthink.com/robby-berman /moose-may-be-the-real-canaries-in-the-coal-mine

Berry A. J. and Gillespie R. G. (2018) Evolution. In G. Baldacchino (ed) The Routledge International Handbook of Island Studies. London: Routledge, pp. 72-100.

Bierregaard Jr. R. O., Lovejoy T. E., Kapos V., dos Santos A. A. and Hutchings R. W. (1992) The Biological Dynamics of Tropical Rainforest Fragments. BioScience, 859-866.

Bird Rose D. (2017a) Shimmer: When all you love is being trashed. In A. L. Tsing, N. Bubandt, E. Gan and H. A. Swanson (eds) Arts of Living on a Damaged Planet: Ghosts and Monsters of the Anthropocene. Minneapolis, MN: University of Minnesota Press, G51-G65.

Bird Rose D. (2017b) Country and the Gift. In J. Adamson and M. Davis (eds) Humanities for the Environment: Integrating Knowledge, Forging New Constellations of Practice. Abingdon: Routledge, pp. 32-45.

Bird Rose D. (2011) Wild Dog Dreaming: Love and Extinction. Charlottesville, VA: University of Virginia Press.

Bongie C. (1998) Islands and Exiles: The Creole Identities of Post/Colonial Literature. Palo Alto, CA: Stanford University Press.

Bonnett A. (2020) The Age of Islands: In Search of New and Disappearing Islands. London: Atlantic Books.

Borges P. A. (2018) Putting Islands on the Map. Conservation Biology, 32(5): 1214-1215. 
Borgnino E. (2020) Mākua Valley: An anthropocenic story of restoration and reconnection, Shima: The International Journal of Research into Island Cultures, 14(2). DOI: https://doi.org/10.21463/shima.14.2.07

Brathwaite K. (2007) DS (2): Dream Stories. New York: New Directions Publishing.

Brathwaite K. (1999) Conversations with Nathaniel Mackey. Staten Island, NY: We Press.

Brathwaite K. (1993) Middle Passages. New York: A New Directions Book. Brathwaite E. (1981) The Arrivants: A New World Trilogy, Rights of Passage, Island Masks. Oxford: Oxford University Press.

Bratton B. H. (2015) The Stack: On Software and Sovereignty. Cambridge, MA: MIT Press.

Breckwoldt A. and Seidel H. (2012) The Need to Know What to Manage: Community-based marine resource monitoring in Fiji. Current Opinion in Environmental Sustainability, 4(3): 331-337.

Bremner L. (2016) Thinking Architecture with an Indian Ocean Aquapelago. GeoHumanities, 2(2): 284-310.

Bridges K. W. and McClatchey W. C. (2009) Living on the Margin: Ethnoecological insights from Marshall Islanders at Rongelap Atoll. Global Environmental Change, 19(2): 140-146.

Briguglio L. and Kisanga, E. J. (eds) (2004) Economic Vulnerability and Resilience of Small States. Malta: Formatek.

Burgess R. L. and Sharpe D. M. (1981) Forest Island Dynamics in Mandominated Landscapes. New York, Heidelberg, Berlin: Springer Verlag.

Burgos Martinez E. (forthcoming) On storiation and what is washed ashore: The Anthropocene as big kahuna. Response to Chandler, D. and Pugh, J. (2021) Anthropocene islands: There are only islands after the end of the world. Dialogues in Human Geography.

Burgos Martinez E. (2020) Theoretical Dancing, Liquidity and Positioning. ESA Network of Contemporary Anthropological Theory. May, 2020. http://www.anthrotheory.net/essays/theoretical-dancing-martinez

Burns L. (2012) Contemporary Caribbean Writing and Deleuze: Literature Between Postcolonialism and Post-continental Philosophy. London: Bloomsbury Publishing.

Burrow Collective (2020) In the Eye of the Storm, Radio from Reina Sofia Museum. Museo Nacional Centro de Arte Reina Sofía. https: //radio.museoreinasofia.es/en/burrow-collective

Cameron F. R. (2011) Saving the 'Disappearing Islands': Climate change governance, Pacific island states and cosmopolitan dispositions. Continuum, 25(6): 873-886. 
Camp E. F., Edmondson J., Doheny A., Rumney J. Grima A. J., Huete A. and Suggett D. J. (2019) Mangrove Lagoons of the Great Barrier Reef Support Coral Populations Persisting Under Extreme Environmental Conditions. Marine Ecology Progress Series, 625: 1-14.

Campbell J. (1984) Dealing with Disaster: Hurricane Response in Fiji. Honolulu, HI: East-West Center.

Camus G. (2018) The Physical and Spiritual Disincarnation of Landscapes under Pressure from Climate Change: Historical and global constraints for Pacific Islanders. In Hessler S. (ed) Tidalectics: Imagining an Oceanic Worldview Through Art and Science. Cambridge, MA: MIT Press, pp. 143-149.

Cantieri J. (2017) Puerto Rico: A 'canary in the coal mine' for climate change, Social Justice News Nexus, 13 January 2017.

Carravieri A., Bustamante P., Churlaud C. and Cherel Y. (2013) Penguins as Bioindicators of Mercury Contamination in the Southern Ocean: Birds from the Kerguelen Islands as a case study. Science of the total environment, 454: 141-148.

Carter P. (2018) Decolonising Governance: Archipelagic Thinking. Routledge: Kindle Edition.

Cass P. (2018) Pacific Islands are Canary in the Coal Mine for Climate Change says Minister, Kaniva Tonga. https://www.kanivatonga.nz /2018/03/pacific-islands-canary-coal-mine-climate-change-says -minister

Cavallo E. (2017) Big Data and the Pursuit of Better Disaster Relief, Ideas Matter, Inter-American Development Bank. https://blogs.iadb .org/ideas-matter/en/big-data-pursuit-better-disaster-relief

Chandler D. (2018a) Ontopolitics in the Anthropocene: An Introduction to Mapping, Sensing and Hacking. Abingdon: Routledge.

Chandler D. (2018b) Digital Governance in the Anthropocene: The rise of the correlational machine. In D. Chandler and C. Fuchs (eds) Digital Objects, Digital Subjects: Interdisciplinary Perspectives on Capitalism, Labour and Politics in the Age of Big Data. London: University of Westminster Press, pp. 23-42.

Chandler D. (2017) Securing the Anthropocene? International policy experiments in digital hacktivism: A case study of Jakarta. Security Dialogue, 48(2): 113-130.

Chandler D. (2014) Resilience: The Governance of Complexity. Abingdon: Routledge.

Chandler D. and Pugh J. (forthcoming, a) Anthropocene Islands: There are only islands after the end of the world. Dialogues in Human Geography. 
Chandler, D. and Pugh, J. (forthcoming, b) Response: The Anthropocene Islands Agenda. Dialogues in Human Geography.

Chandler D. and Pugh J. (2020a) Islands of Relationality and Resilience: The shifting stakes of the Anthropocene. Area, 52(1): 65-72.

Chandler D. and Pugh J. (2020b) Islands and the Rise of Correlational Epistemology in the Anthropocene: Rethinking the trope of the 'canary in the coalmine'. Island Studies Journal. E-pub ahead of print DOI: https://doi.org/10.24043/isj.119

Chandler D. and Reid J. (2018) 'Being in Being': Contesting the ontopolitics of indigeneity. The European Legacy, 23: 251-268.

Chao S. (2020) Nature in Culture: Multi-sensory mapping with the Marind people. Sidney Environmental Institute. https://sei.sydney.edu .au/publications/nature-in-culture-multi-sensory-mapping

Clark E. and Tsai H. M. (2009) Ecologically unequal exchange and landesque capital on Kinmen Island. Asia-Pacific Forum, 44: 148-167.

Clark N. (n.d.) Shrinking Worlds: Islands, interconnectivity, climate change. http://physicsroom.org.nz/archive/shrink/essay/2.htm?fbclid =IwAR1YTO4_7XmU_11XUwM6D9mgbU3VDhT61kNI13zhm XpnGm4GWkqHrThQAGU

Clark N. and Szerszynski B. (2021) Planetary Social Thought: The Anthropocene challenge to the social sciences. Cambridge. Polity.

Clark N. and Yusoff K. (2017) Geosocial Formations and the Anthropocene. Theory, Culture \& Society, 34(2-3): 3-23.

Cole D. R., Dolphijn R. and Bradley J. P. (2016) Fukushima: The geotrauma of a futural wave. Trans-Humanities Journal, 9(3): 211-233.

Colebrook C. (forthcoming) No Man is an Island. Response to Chandler D. and Pugh J. (forthcoming) Anthropocene Islands: There are only islands after the end of the world. Dialogues in Human Geography.

Colebrook C. (2019) A Cut in Relationality: Art at the end of the world. Angelaki, 24(3): 175-195.

Colebrook C. (2016) What is the Anthropo-Political? In T. Cohen, J. Hillis Miller and C. Colebrook, Twilight of the Anthropocene Idols. Open Humanities Press, pp. 81-117.

Colebrook C. and Weinstein J. (2017) Preface: Postscript on the posthuman. In J. Weinstein and C. Colebrook (eds) Posthumous Life: Theorizing Beyond the Posthuman. New York: Columbia University Press, pp. xi-xxvii.

Condé M. (1992) Tree of Life. New York: Ballantine Books.

Connell J. (2018) Migration. In G. Baldacchino (ed) The Routledge International Handbook of Island Studies: A World of Islands. London: Routledge, pp. 261-279. 
CORDIS (2018) A Remote Greek Island is on its Way to Becoming Energy Self-sufficient, Phys.org. https://phys.org/news/2018-10-remote -greek-island-energy-self-sufficient.html

Crane R. and Fletcher L. (2017) Island Genres, Genre Islands: Conceptualisation and Representation in Popular Fiction. New York: Rowman and Littlefield.

Cyphers P. (2019) Anthropocene Island: Colonization, native species and invaders. Pratt Institute Fine Art. https://commons.pratt.edu /prattfinearts/anthropocene-island

Danowski D. and Viveiros de Castro E. (2016) The Ends of the World. Cambridge: Polity.

Daou D. and Pérez-Ramos P. (2016) (eds) New Geographies 08: Island. Cambridge, MA: Harvard University Graduate School of Design.

Darwin C. (2010) The Works of Charles Darwin, Volume 16: The Origin of Species, 1876. New York: NYU Press.

Dash M. J. (2006) Farming Bones and Writing Rocks: Rethinking a Caribbean poetics of (dis)location. Shibboleths: Journal of Comparative Theory, 1(1): 64-71.

Davis H. and Turpin E. (2015) Art in the Anthropocene: Encounters among Aesthetics, Politics, Environments and Epistemologies. Open Humanities Press.

Davis S. (forthcoming) Islands, Modernity and Other Worlds that Never End. Response to Chandler D. and Pugh J. (forthcoming) Anthropocene Islands: There are only islands after the end of the world. Dialogues in Human Geography.

Davis S. (2020) Islands and Oceans: Reimagining Sovereignty and Social Change. Athens, GA: University of Georgia Press.

Dawson H. (2019) Island Archaeology. In C. Smith (ed) Encyclopedia of Global Archaeology. Cham: Springer. DOI: https://doi.org/10 .1007/978-3-319-51726-1

da Silva D. F. (in conversation with Winterling S. M.) (2017) Fractal Sensing/thinking on a Planetary scale. Planetary Sensing. https: //planetarysensing.com/fractal-sensing-thinking-on-a-planetary -scale

da Silva D. F. (2016) On Difference Without Separability. Catalogue of the 32a São Paulo Art Biennial, 'Incerteza viva' (Living Uncertainty). https://issuu.com/amilcarpacker/docs/denise_ferreira_da_silva

Deleuze G. (2004) Desert Islands and Other Texts, 1953-1974. Los Angeles, CA: Semiotext(e).

Deleuze G. and Guattari F. (1986) Kafka: For a Minor Literature. Minneapolis, MN: University of Minnesota Press. 
DeLoughrey E. M. (2019) Allegories of the Anthropocene. Durham, NC: Duke University Press.

DeLoughrey E. (2010) Heavy waters: Waste and Atlantic modernity. PMLA, 125(3): 703-712.

DeLoughrey E. M. (2007) Routes and Roots: Navigating Caribbean and Pacific island literatures. Honolulu, HI: University of Hawai'i Press.

DeLoughrey E. and Flores T. (2020) Submerged Bodies: The tidalectics of representability and the sea in Caribbean art. Environmental Humanities, 12(1): 132-166.

Dening G. (2007) Sea People of the West. Geographical Review, 97(2): 288-301.

Depraetere C. (2008) The Challenge of Nissology: A global outlook on the world archipelago part I: Scene setting the world archipelago. Island Studies Journal, 3(1): 3-16.

Derissen S., Quaas M. F. and Baumgärtner S. (2011) The Relationship between Resilience and Sustainability of Ecological-economic Systems. Ecological Economics, 70(6): 1121-1128.

Derrida J. (2011) The Beast and the Sovereign (Vol. 2). Chicago, IL: University of Chicago Press.

De Souza R. M., Henly-Shepard S., McNamara K. and Fernando N. (2015) Re-framing Island Nations as Champions of Resilience in the Face of Climate Change and Disaster Risk. UNU-EHS Working Paper Series, No. 17. Bonn: United Nations University Institute of Environment and Human Security. http://collections.unu.edu/eserv /UNU:2856/Reframing_island_nations_WP_No_17.pdf

de Sousa Santos B. (2016) Epistemologies of the South: Justice against Epistemicide. Abingdon: Routledge.

De Waal A. (2020) New Pathogen, Old Politics. Boston Review: A political and literary forum, $3^{\text {rd }}$ April, 2020. http://bostonreview.net/science -nature/alex-de-waal-new-pathogen-old-politics

DeWeerdt S. (2019) Does an Island Lifestyle Confer Climate Toleranceif you're a Plant? Anthropocene. https://www.anthropocenemagazine .org/2019/07/island-lifestyle-climate-tolerance-plant

Dodds K. and della Dora V. (2018) Artificial Islands and Islophilia. In G. Baldacchino (ed) The Routledge International Handbook of Island Studies: A World of Islands. New York: Routledge, pp. 392-416.

Drabinski J. E. (2019) Glissant and the Middle Passage: Philosophy, Beginning, Abyss. Minneapolis, MN: University of Minnesota Press.

Drifters Project (2019) Anthropocene Islands, Les Gallery @ The Clemente, NY! Drifters Project, 17 February. https://driftersproject 
.net/anthropocene-island-les-gallery-at-the-clemente-ny-coming -soon

Earth Island. (2019). FY 2019 Annual Report. Earth Island Institute, Berkley: California https://www.earthisland.org/assets/2019Annual Report.pdf

ecoLogicStudio (2017a) bioTallin: Biocities. http://www.ecologicstudio .com/v2/project.php?idcat $=7 \&$ idsubcat $=71 \&$ idproj $=166$

ecoLogicStudio (2017b) TAB 2017 Curated Exhibition: Anthropocene Island. http://2017.tab.ee/article/tab-2017-anthropocene-island ecoLogicStudio (2017c) Anthropocene Island: the TAB 2017 curated exhibition. https://www.arhitektuurimuuseum.ee/en/naitus/anthro pocene-island-the-tab-2017-curated-exhibition

Edmond R. S. and Smith V. (2003) Islands in History and Representation. Abingdon: Routledge.

Edwards P. J., Kollmann J., Gurnell A. M., Petts G. E., Tockner K. and Ward J. V. (1999) A Conceptual Model of Vegetation Dynamics on Gravel Bars of a Large Alpine River. Wetlands Ecology and Management, 7(3): 141-153.

Edwards P. N. (2010) A Vast Machine: Computer Models, Climate Data, and the Politics of Global Warming. Cambridge, MA: MIT Press.

Elias A. (2019) Coral Empire: Underwater Oceans, Colonial Tropics, Visual Modernity. Durham, NC: Duke University Press.

Ellsmoor J. (2020) The Virtual Island Summit. Island Innovation. https://www.islandinnovation.co/summit

Ellsmoor J. (2019) Understanding Resilience means Understanding the Role of Indigenous Communities: Indigenous communities can inspire resilient solutions, but their burden should be shared. Understanding Risk, https://understandrisk.org/understanding-resilience -means-understanding-the-role-of-indigenous-communities $\% \mathrm{EF} \% \mathrm{BB}$ $\% \mathrm{BF}$-indigenous-communities-can-inspire-resilient-solutions-but-their -burden-should-be-shared-\%EF\%BB\%BF

Enn R. (2015) Governance, Empowerment, and Environmental Justice, Doctoral dissertation. Universität Wien.

Eriksen T. H. (1993) In Which Sense do Cultural Islands Exist? Social Anthropology, 1: 133-147.

European Union (2019) 3rd Clean Energy EU Islands Forum, Clean Energy for EU Islands Scretariat. https://www.euislands.eu/clean -energy-islands-start

Evans B. and Reid J. (2014) Resilient Life: The Art of Living Dangerously. Cambridge: Polity. 
Evans M. and Harris L. (2018) Salmon as Symbol, Salmon and Guide: What anadromous fish can do for thinking about islands, ecosystems and the globe. Shima: The International Journal of Research into Island Cultures, 12(1): 1-14.

Fair H. (2018) Three Stories of Noah: Navigating religious climate change narratives in the Pacific Island region. Geo: Geography and Environment, 5(2), e00068.

Falefou T. (2017) Toku Tia: Tuvalu and the Impacts of Climate Change. Doctoral dissertation, University of Waikato.

Fanon F. (1967) The Wretched of the Earth. London: Penguin Books.

Farbotko C. (2018a) Climate Change and Island Populations. In B. J. Steele and E. A. Heinze (eds) Routledge Handbook of Ethics and International Relations. Abingdon: Routledge, pp. 405-418.

Farbotko C. (2018b) Voluntary Immobility: Indigenous voices in the Pacific. Forced Migration Review, 57: 81-83.

Farbotko C. (2010) Wishful Sinking: Disappearing islands, climate refugees and cosmopolitan experimentation. Asia Pacific Viewpoint, 51(1): 47-60.

Faris J. H. (2019) Sister of Ocean and Ice: On the hydro-feminism of Kathy Jetnil-Kijiner and Aka Niviâna's Rise: From one island to another. Shima: The International Journal of Research into Island Cultures, 13(2). DOI: https://doi.org/10.21463/shima.13.2.08

Farrier D. (2020) Footprints: In search of fossil futures. London: 4th Estate.

Farrier D. (2019) Anthropocene Poetics: Deep Time, Sacrifice Zones, and Extinction. Minneapolis, MN: University of Minnesota Press.

Farrier D. and Dickenson A. (2020) Episode 6: Anthropocene Poetics: David Farrier with Adam Dickinson. Soundcloud. https://sound cloud.com/user-760891605/farrier

Filmproduktion and Arte G.E.I.E. (2016) Islands of the Future, Netflix TV Series.

Finney B. (2003) Sailing in the Wake of the Ancestors: Reviving Polynesian Voyaging. Honolulu, HI: Bishop Museum Press.

First Peoples Worldwide (n.d.) Who are Indigenous Peoples: How Our Societies Work. First People's Worldwide. http://www.firstpeoples .org/how-our-societies-work.htm

Fish A. (2019) Networking Nature: Tracking terra, sensing the sea, atmostructures. Anthro-Dendum. https://anthrodendum.org/2019/07/10 /networking-nature-tracking-terra-sensing-the-sea-atmo-structures

Fitzpatrick S. M. and Erlandson J. M. (2018) Island Archaeology, Model Systems, the Anthropocene, and How the Past Informs the Future. Journal of Island and Coastal Archaeology, 13(2): 283-299. 
Flores T. and Stephens M. A. (eds) (2017) Relational Undercurrents: Contemporary Art of the Caribbean Archipelago. Durham, NC: Duke University Press.

Foo S. A. and Asner G. P. (2019) Scaling Up Coral Reef Restoration Using Remote Sensing Technology, Frontiers in Marine Science, 13 March 2019. https://www.frontiersin.org/articles/10.3389/fmars .2019.00079/full

Forest Peoples Programme (2019) Islands of Nature in a Sea of Decline: Indigenous and local knowledge, action and contributions key to saving the world's nature. Forest Peoples Programme, 6 May 2019. https://www.forestpeoples.org/en/international-processes-convention -biological-diversity-cbd/press-release/2019/media-release

Gaini F. and Nielsen H. P. (eds) (2020) Gender and Island Communities. Abingdon: Routledge.

Galle N. (2017) Islands as Living Labs for Circular Economics, Metabolic. https://www.metabolic.nl/news/islands-living-labs-circular -economics

Gane M. (1975) Report of a Mission to Assess the Hurricane Factor for Planning Purposes in Fiji. Bradford Disaster Research Unit Occasional Paper 9. Bradford: University of Bradford.

Genz J. (2011) Navigating the Revival of Voyaging in the Marshall Islands: Predicaments of Preservation and Possibilities of Collaboration. The Contemporary Pacific, 23(1): 1-34.

Ghosh A. (2016) The Great Derangement: Climate Change and the Unthinkable. London: University of Chicago Press.

Ghosh S. (2020) How Rhizophora Mangroves on Car Nicobar Islands Fought Back a Rapid Sea-level Rise in 2004 Tsunami, Mongabay, 27 August 2020. https://india.mongabay.com/2020/08/how-rhizophora -mangroves-on-car-nicobar-islands-fought-back-a-rapid-sea-level -rise-in-2004-tsunami

Gillis J. R. (2004) Islands of the Mind: How the Human Imagination Created the Atlantic World. New York: Palgrave Macmillan.

Giraud E. H. (2019) What Comes After Entanglement? Activism, Anthropocentrism, and an Ethics of Exclusion. Durham, NC: Duke University Press.

Glissant É. (1997) Poetics of Relation. Ann Arbor, MI: University of Michigan Press.

Goffman E. (1978) The Presentation of Self in Everyday Life. Penguin: Harmondsworth.

Gómez-Barris M. and Joseph M. (2019) Introduction: Coloniality and islands. Shima: The International Journal of Research into Island 
Cultures, 13(2) e-pub ahead of print, DOI: https://doi.org/10.21463 /shima.13.2.03

González J. A., Montes C., Rodríguez J. and Tapia W. (2008) Rethinking the Galapagos Islands as a Complex Social-ecological System: Implications for conservation and management. Ecology and Society 13(2): 13. [online]: http://www.ecologyandsociety.org/vol13/iss2/art13

Gordon L. (2018) What Does the End of the World Sound Like? Listen to this. The Outline, https://theoutline.com/post/5960/climate -change-music?zd=2\&zi=6j7 wips5

Graham N. R., Gruner D. S., Lim J. Y. and Gillespie R. G. (2017) Island Ecology and Evolution: Challenges in the Anthropocene. Environmental Conservation, 44(4): 323-335.

Graziadei D., Hartmann B., Kinane I., Riquet J. and Samson, B. (2017) On Sensing Island Spaces and the Spatial Practice of Island -making: Introducing island poetics, Part I. Island Studies Journal, 12(2): 239-253.

Grosz E. (2004) The Nick of Time: Politics, Evolution, and the Untimely. Durham, NC: Duke University Press.

Grove K. (forthcoming) Islands of (In)Security in the Anthropocene. Response to Chandler D. and Pugh J. (forthcoming) Anthropocene Islands: There are only islands after the end of the world. Dialogues in Human Geography.

Grove K. (2018) Resilience. Abingdon: Routledge.

Grove K., Krivý M., Rickards L., Schliwa G., Collier S. J., Cox S. and Gandy M. (2019) Interventions on Design and Political Geography. Political Geography, 74, 102017. E-pub ahead of print. DOI: https://doi .org/10.1016/j.polgeo.2019.04.009

Grove R. (1995) Green Imperialism: Colonial Expansion, Tropical Island Edens and the Origins of Environmentalism. Cambridge: Cambridge University Press.

Grydehøj A. (2017) A future of Island Studies. Island Studies Journal, 12(1): 3-16.

Grydehøj A. and Kelman I. (2020) Reflections on Conspicuous Sustainability: Creating Small Island Dependent States (SIDS) through Ostentatious Development Assistance (ODA)? Geoforum, 116: 90-97.

Grydehøj A. and Kelman I. (2017) The Eco-island Trap: Climate change mitigation and conspicuous sustainability. Area, 49: 106-113.

Grydehøj A. and Kelman I. (2016) Island Smart Eco-cities: Innovation, secessionary enclaves, and the selling of sustainability. Urban Island Studies, 2: 1-24. 
Gumbs A. P. (2018) M Archive: After The End of the World. Durham, NC: Duke University Press.

Gunderson L. H. (2001) South Florida: The reality of change and the prospects for sustainability: Managing surprising ecosystems in Southern Florida. Ecological Economics, 37(3): 371-378.

Gunderson L. H., Allen C. R. and Holling C. S. (eds) (2010) Foundations of Ecological Resilience. Washington, DC: Island Press.

Günel G. (2019) Spaceship in the Desert: Energy, Climate Change, and Urban Design in Abu Dhabi. Durham, NC: Duke University Press.

Günel G., Varma S. and Watanabe C. (2020) A Manifesto for Patchwork Ethnography. Member Voices, Fieldsights, 9 June 2020. https: //culanth.org/fieldsights/a-manifesto-for-patchwork-ethnography

Gutierrez L. and Portefaix V. (2015) Islands and other Invisible Territories. In H. Davis and E. Turpin (eds) (2015) Art in the Anthropocene: Encounters among Aesthetics, Politics, Environments and Epistemologies. Open Humanities Press, pp. 223-233.

Hadfield M. G. and Haraway D. J. (2019) The Tree Snail Manifesto. Current Anthropology, 60(20): 209-235.

Hall E. F. and Sanders T. (2015) Accountability and the Academy: Producing knowledge about the human dimensions of climate change. Journal of the Royal Anthropological Institute, 21(2): 438-461.

Hallward P. (2001) Absolutely Postcolonial: Writing Between the Singular and the Specific. Manchester: Manchester University Press.

Hamilton S. (2017) Securing Ourselves from Ourselves? The paradox of 'entanglement' in the Anthropocene. Crime, Law and Social Change, 68(5): 579-595.

Handforth D. (2020) How Can Small States Benefit from Smart City Innovation? Island Innovation. https://www.islandinnovation.co/how -can-small-states-benefit-from-smart-city-innovation

Handley G. B. (2015) Climate Change, Cosmology, and Poetry: The case of Derek Walcott's Omeros. In E. DeLoughrey, J. Didur and A. Carrigan (eds) Global Ecologies and the Environmental Humanities: Postcolonial Approaches. New York: Routledge, pp. 333-352.

Hanna E. G. and McIver L. (2014) Small Island States: Canaries in the coal mine of climate change and health. In C. D. Butler (ed) Climate Change and Global Health. Wallingford: CABI, pp. 181-192.

Haraway D. J. (2016) Staying with the Trouble: Making Kin in the Chthulucene. Durham, NC: Duke University Press.

Haraway D. J. (2008) When Species Meet. Minneapolis, MN: University of Minnesota Press. 
Harford T. (2011) Adapt: Why Success Always Starts with Failure. London: Little, Brown.

Harney S. and Moten F. (2013) The Undercommons: Fugitive Planning and Black Study. Wivenhoe, New York and Port Watson: Minor Compositions.

Hartman S. V. (1997) Scenes of Subjection: Terror, Slavery, and Self-Making in Nineteenth-Century America. Oxford: Oxford University Press.

Hau'ofa E. (2008) We Are the Ocean. Honolulu, HI: University of Hawai'i Press.

Hawkins H. (2018) 'A Volcanic Incident': Towards a geopolitical aesthetics of the subterranean. Geopolitics, 24(3): 1-26.

Hay P. (2013) What the Sea Portends: A reconsideration of contested island tropes. Island Studies Journal, 8(2): 209-232.

Hay P. A. (2006) Phenomenology of Islands. Island Studies Journal, 1(1): $19-42$.

Hayward P. (2018) Keynote: Formulations in Flux. Archipelagos and Aquapelagos - Conceptualising Islands and Marine Spaces conference, Pratt Institute, Brooklyn, 30 March-1 April.

Hayward P. (2012a) Aquapelagos and Aquapelagic Assemblages. Shima: The International Journal of Research into Island Cultures, 6(1): 1-11.

Hayward P. (2012b) The Constitution of Assemblages and the Aquapelagality of Haida Gwaii. Shima: The International Journal of Research into Island Cultures, 6(2): 1-8.

Hejnol A. (2017) Ladders, Trees, Complexity, and Other Metaphors in Evolutionary Thinking. In A. L. Tsing, N. Bubandt, E. Gan and H. A. Swanson (eds) Arts of Living on a Damaged Planet: Ghosts and monsters of the Anthropocene. Minneapolis, MN: University of Minnesota Press, G87-103.

Helmus M. R., Mahler D. L. and Losos J. B. (2014) Island Biogeography of the Anthropocene. Nature, 513(7519): 543-546.

Hennessy E. (2019) On the Backs of Tortoises: Darwin, the Galápagos, and the Fate of an Evolutionary Eden. New Haven, CT: Yale University Press.

Herrington S. and Lokman K. (2016) Gardens as Migratory Devices. In D. Daou and P. Pérez-Ramos (eds) New Geographies 08: Island. Cambridge, MA: Universal Wilde, pp. 142-154.

Hessler S. (2018) (ed) Tidalectics: Imagining an Oceanic Worldview Through Art and Science. Cambridge, MA: MIT Press.

His Highness Tui Atua Tupua Tamasese Ta'isi Efi (2018) Prelude: Climate Change and the Perspective of the Fish. In T. Crook and 
P. Rudiak-Gould (eds) Pacific Climate Cultures: Living Climate Change in Oceania. De Gruyter Open, IX-XIII.

Holbraad M. and Pedersen M. A. (2017) The Ontological Turn: An Anthropological Exposition. Cambridge: Cambridge University Press. Holdaway S. J., Emmitt J., Furey L., Jorgensen A., O’ Regan G., Phillipps R., Prebble M., Wallace R. and Ladefoged T. N. (2019) Māori Settlement of New Zealand: The Anthropocene as a process. Archaeology in Oceania, 54(1): 17-34.

Holland J. H. (1998) Emergence: From Chaos to Order. Oxford: Oxford University Press.

Holling C. S. (1973) Resilience and Stability of Ecological Systems. Annual Review of Ecology and Systematics, 4(1): 1-23.

Hong G. (2017) Locating Zhuhai Between Land and Sea: A relational production of Zhuhai, China, as an island city. Island Studies Journal, 12(2): 7-24.

Howe R. W. (1984) Local Dynamics of Bird Assemblages in Small Forest Habitat Islands in Australia and North America. Ecology, 65(5): 1585-1601.

Huang H. (2017) Performing Archipelagic Identities in Bill Reid, Robert Sullivan and Syaman Rapongan. In B. R. Roberts and M. A. Stephens (eds) Archipelagic American Studies. Durham, NC: Duke University Press, pp. 281-302.

Iida S. and Nakashizuka T. (1995) Forest Fragmentation and its Effect on Species Diversity in Sub-urban Coppice Forests in Japan. Forest Ecology and Management, 73(1-3): 197-210.

Ingersoll K. A. (2016) Waves of Knowing: A Seascape Epistemology. Durham, NC: Duke University Press.

Ingold T. (2015) The Life of Lines. Abingdon: Routledge.

Intergovernmental Panel on Climate Change (2007) Climate Change 2007: Synthesis Report. Contribution of Working Groups I, II and III to the Fourth Assessment Report of the Intergovernmental Panel on Climate Change [Core Writing Team, Pachauri R. K. and Reisinger A. (eds)]. Geneva, Switzerland: Intergovernmental Panel on Climate Change. http://www.ipcc.ch/publications_and_data/publications_ipcc _fourth_assessment_report_synthesis_report.htm

Isaacs J. R. (2020) Conservation Archipelago: Protecting long-distance migratory shorebirds along the Atlantic flyway. In M. Stephens and Y. Martínez-San Miguel (eds) Contemporary Archipelagic Thinking: Toward New Comparative Methodologies and Disciplinary Formations. Lanham, MD: Rowman and Littlefield. 
Jetñil-Kijiner K. (2019) Bulldozed Reefs and Blasted Sands: Rituals for artificial islands. Kathy Jetñil-Kijiner's website. https://www.kathyjet nilkijiner.com/bulldozed-reefs-and-blasted-sands-rituals-for-artificial -islands

Johnson E. R. (2017) At the Limits of Species Being: Sensing the Anthropocene. South Atlantic Quarterly, 116(2): 275-292.

Johnson J. M. (2019) Xroads Praxis: Black diasporic technologies for remaking the New World. sx archipelagos: A small axe platform for digital practice. http://smallaxe.net/sxarchipelagos/issue03/johnson .html

Johnson S. (2001) Emergence. London: Penguin.

Jolly M. (2007) Imagining Oceania: Indigenous and foreign representations of a sea of islands. The Contemporary Pacific, 19(2): 508-545.

Jongejans E. (2019) Automatic Insect Identification for Better Grasp on Biodiversity. Phys.org, 24 May 2019. https://phys.org/news/2019-05 -automatic-insect-identification-grasp-biodiversity.html

Jørgensen P. S., Folke C. and Carroll S. P. (2019) Evolution in the Anthropocene: Informing governance and policy. Annual Review of Ecology, Evolution, and Systematics, 50: 52-46.

Joseph J. (2013) Resilience as Embedded Neoliberalism: A governmentality approach. Resilience, 1(1): 38-52.

Joseph M. (2020) Islands, History, Decolonial Memory. Island Studies Journal, 15(2): 193-200.

Joseph M. (2019) Sea Log: Indian Ocean to New York. Abingdon: Routledge. Joseph M. (2013) Fluid New York: Cosmopolitan Urbanism and the Green Imagination. Durham, NC: Duke University Press.

Jue M. (2020) Wild Blue Media: Thinking through Seawater. Durham, NC: Duke University Press.

Karides M. (2017) Why Island Feminism? Shima: The International Journal of Research into Island Cultures, 11(1): 30-39.

Karides M. (2016) An Island Feminism: Convivial economics and the women's cooperatives of Lesvos. In E. Stratford (ed) Island Geographies: Essays and Conversations. Abingdon: Routledge, pp. 78-97.

Kanngieser A. (2020) Listening to Ecocide. https://www.youtube.com /watch? $=$ dh1Cs4G7mxk

Kanngieser A. and Todd Z. (2020) From Environmental Case Study to Environmental Kin Study. History and Theory, 59(3): 385-393.

Kearns R. and Collins D. (2016) Aotearoa's Archipelago: Re-imagining New Zealand's island geographies. New Zealand Geographer, 72(3): 165-168. 
Keim M. (2019) Small Islands are the Canaries in the Climate-crisis Coal Mine. Disaster Doc, 6 February 2019. https://disasterdoc.org /small-islands-canaries-climate-crisis-coal-mine/

Kelly D. and Lobo M. (2020) Tidal Country and Cultures in Northern Australia. In A. Campbell, M. Duffy and B. Edmondson (eds) Located Research. Singapore: Palgrave Macmillan, pp. 303-318.

Kelman I. (2020) Islands of Vulnerability and Resilience: Manufactured stereotypes? Area, 52(1): 6-13.

Kelman I (2018) Islandness Within Climate Change Narratives of Small Island Developing States (SIDS). Island Studies Journal, 13(1): 149-166.

Kelman I. and Randall J. E. (2018) Resilience and Sustainability. In G. Baldacchino (ed) The Routledge International Handbook of Island Studies: A World of Islands. Abingdon: Routledge, pp. 353-368.

Kimmerer R. W. (2013) Braiding Sweetgrass: Indigenous Wisdom, Scientific Knowledge and the Teachings of Plants. Minneapolis, MN: Milkweed Editions.

King R. (2009) Geography, Islands and Migration in an Era of Global Mobility. Island Studies Journal, 4(1): 53-84.

King T. L. (2019) The Black Shoals: Offshore Formations of Black and Native Studies. Durham, NC: Duke University Press.

Kirby V. (2011) Quantum Anthropologies: Life at Large. Durham, NC: Duke University Press.

Kirksey E. (2020) Chemosociality in Multispecies Worlds: Endangered Frogs and Toxic Possibilities in Sydney. Environmental Humanities, 12(1): 23-50.

Kohn E. (2013) How Forests Think: Toward an Anthropology Beyond the Human. Berkeley, CA: University of California Press.

Kueffer C. and Kaiser-Bunbury C. N. (2014) Reconciling Conflicting Perspectives for Biodiversity Conservation in the Anthropocene. Frontiers in Ecology and the Environment, 12(2): 131-137.

Kueffer C. and Kinney K. (2017) What is the Importance of Islands to Environmental Conservation? Environmental Conservation, 44(4):311-322.

Kurki M. (2020) International Relations in a Relational Universe. Oxford: Oxford University Press.

Lama P. D. (2018) Gendered Consequences of Mobility for Adaptation in Small Island Developing States: Case studies from Maafushi and Kudafari in the Maldives. Island Studies Journal, 13(2): 111-128.

Larjosto V. (2020) Islands of the Anthropocene. Area, 52(1): 38-46.

Last A. (2017) We are the World? Anthropocene cultural production between geopoetics and geopolitics. Theory, Culture \& Society, 34(2-3): $147-168$. 
Latour B. (2017) Facing Gaia: Eight Lectures on the New Climatic Regime. Cambridge: Polity.

Latour B. (2013) Facing Gaia: Six Lectures on the Political Theology of Nature: Being the Gifford Lectures on Natural Religion, Edinburgh, 18th-28th of February 2013 (draft version 01-03-2013).

Latour B. (2005) Reassembling the Social: An Introduction to Actor -Network-Theory. Oxford: Oxford University Press.

Latour B. (2004) How to Talk About the Body? The normative dimension of science studies. Body \& Society, 2-3: 205-229.

Latour B. (1993) We Have Never Been Modern. Cambridge, MA: Harvard University Press.

Latour B., Harman G. and Erdélyi P. (2011) The Prince and the Wolf: Latour and Harman at the LSE. Winchester: Zero Books.

Law J. (2007) Actor Network Theory and Material Semiotics. In B. S. Turner (ed) The New Blackwell Companion to Social Theory, 3rd Edition. Oxford: Blackwell, pp. 141-158.

Leppard T. P. (2018) Rehearsing the Anthropocene in Microcosm: The palaeoenvironmental impacts of the Pacific rat (Rattus exulans) and other non-human species during island neolithization. In S. E. P. Birch (ed) Multispecies Archaeology. Abingdon: Routledge, pp. 47-64.

Lewis D. (1994) We, the Navigators: The Ancient Art of Landfinding in the Pacific. Honolulu, HI: University of Hawai'i Press.

Lewis J. (1984) A Multi-hazard History of Antigua. Disasters, 8(3): 190-197. Li S., Yu K., Chen T., Shi Q. and Zhang H. (2011) Assessment of Coral Bleaching using Symbiotic Zooxanthellae Density and Satellite Remote Sensing data in the Nansha Islands, South China Sea. Chinese Science Bulletin, 56(10): 1031-1037.

Lovelock J. (2020) Novacene. London: Penguin.

Lovelock J. (2007) The Revenge of Gaia. London: Penguin.

Loughran G. (2019) Day 1 Guayaquil Archipelago Conference, $11^{\text {th }}$ February 2019. https://www.youtube.com/watch?v=7iDpNm6F4PA Louis R. P. and Kahele M. (2017) Kanaka Hawai'i Cartography: Hula, Navigation, and Oratory. Corvallis, OR: Oregon State University Press. MacArthur R. H. and Wilson E. O. (1967/2001) The Theory of Island Biogeography. Princeton, NJ: Princeton University Press.

Macfarlane R. (2019) Underland: A Deep Time Journey. London: Penguin. Machine Wilderness (n.d.) An Art/science Programme Exploring how Human Technologies could Relate to the Environment the Way Organisms Do. Machine Wilderness. http://machinewilderness.net/ 
Māhina H. O. (2008) From Vale (Ignorance) to Ilo (Knowledge) to Poto (skill) the Tongan Theory of Ako (education): Theorising old problems anew. AlterNative: An International Journal of Indigenous Peoples, 4(1): 67-96.

Malinowski B. (1921) The Primitive Economics of the Trobriand Islanders. The Economic Journal, 31(121): 1-16.

Mann T. and Westphal H. (2014) Assessing Long-Term Changes in the Beach Width of Reef Islands Based on Temporally Fragmented Remote Sensing Data. Remote Sensing, 6(8): 6961-6987.

Marland P. (2014) Island of the Dead: Composting twenty-thousand saints on Bardsey Island. Green Letters, 18(1): 78-90.

Marques P. N. (2017) Mimetic Traps: Forests, images, worlds. In E. Turpin and A.-S. Springer (eds) Intercalations 04: The Word for World is Still Forest. Berlin: Haus der Kulturen der Welt and K. Verlag, pp. 21-38.

Martínez-San Miguel Y. (2014) Coloniality of Diasporas: Rethinking Intra-colonial Migrations in a Pan-Caribbean Context. New York: Palgrave Macmillan.

Marx K. (1973) Gundrisse: Foundations of the Critique of Political Economy. Harmondsworth: Penguin.

Massumi B. (2019) Architectures of the Unforeseen: Essays in the Occurrent Arts. Minnesota, MN: University of Minnesota Press.

Mathews A. (2017) Ghostly Forms and Forest Histories. In A. L. Tsing, N. Bubandt, E. Gan and H. A. Swanson (eds) Arts of Living on a Damaged planet: Ghosts and Monsters of the Anthropocene. Minneapolis, MN: University of Minnesota Press, G145-G157.

Mathews T. J., Rigal F., Triantis K. A. and Whittaker R. J. (2019) A Global Model of Island Species-area Relationships. Proceedings of the National Academy of Sciences, 116(25): 12337-12342.

McCall G. (1994) Nissology: A proposal for consideration. 太平洋学会 学会誌, (63): 93-106.

McKittrick K. (2006) Demonic Grounds: Black Women and the Cartographies of Struggle. Minnesota, MN: University of Minnesota Press.

McMahon E. (2016) Islands, Identity and the Literary Imagination. London: Anthem Press.

McMillen H. L., Ticktin T., Friedlander A., Jupiter S. D., Thaman R., Campbell J., Veitayaki J., Giambelluca T., Nihmei S., Rupeni E. and Apis-Overhoff L. (2014) Small Islands, Valuable Insights: Systems of customary resource use and resilience to climate change in the Pacific. 
Ecology and Society, 19(4): 44. DOI: http://doi.org/10.5751/ES-06937 $-190444$

Mead M. (2001) The Coming of Age in Samoa. New York: Harper Perennial.

Mead M. (1957) Introduction to Polynesia as Laboratory for the Development of Models in the Study of Cultural Evolution. Journal of the Polynesian Society, 66: 145.

Mentz S. (2017) Enter Anthropocene, Circa 1610. In T. Menely and J. O. Taylor (eds) Anthropocene Reading: Literary History in Geologic Times (Vol. 1). University Park, PA: Penn State Press, pp. 43-59.

Merola N. M. (2018) 'What Do We Do but Keep Breathing as Best We can this/Minute Atmosphere': Juliana Spahr and Anthropocene Anxiety. In K. Bladow and J. Ladino (eds) Affective Ecocriticism: Emotion, Embodiment, Environment. Lincoln, NE: University of Nebraska Press, pp. 25-51.

Mika K. (2018) Disasters, Vulnerability, and Narratives: Writing Haiti's Futures. Abingdon: Routledge.

Mission Blue. (2019) First Ever Hope Spot in Mainland United Kingdom Declared Along Scotland's Argyll Coast and Islands. Mission Blue: Sylvia Earle Alliance. 4 June 2019. https://mission-blue.org/2019/06 /first-ever-hope-spot-in-mainland-united-kingdom-declared-along -scotlands-argyll-coast-and-islands

Mitchell J. (1978) Amokura. Auckland: Longman Paul.

Mohanty P. C., Mahendra R. S., Bisoyi H., Tummula S. K., Grinson G., Nayak S. and Sahu B. K. (2013) Assessment of the Coral Bleaching During 2005 to Decipher the Thermal Stress in the Coral Environs of the Andaman Islands using Remote Sensing. European Journal of Remote Sensing, 46(1): 417-430.

Moore A. (2020) Introduction: Islands and aquapelagos in the Anthropocene, Shima: The International Journal of Research into Island Cultures, 14(2). DOI: https://doi.org/10.21463/shima.14.2.03

Moore A. (2019a) Destination Anthropocene: Science and Tourism in the Bahamas. Oakland, CA: University of California Press.

Moore A. (2019b) Selling Anthropocene Space: Situated adventures in sustainable tourism. Journal of Sustainable Tourism, 27(4): 436-451.

Moore A. (2016) Anthropocene Anthropology: Reconceptualizing contemporary global change. Journal of the Royal Anthropological Institute, 22(1): 27-46.

Moore A. (2015a) Islands of Difference: Design, urbanism, and sustainable tourism in the Anthropocene Caribbean. Journal of Latin American and Caribbean Anthropology, 20(3): 513-532. 
Moore A. (2015b) Tourism in the Anthropocene Park? New analytic possibilities. International Journal of Tourism Anthropology, 4(2): 186-200.

Morton T. (2017) Spectral Life: The uncanny valley is in fact a gigantic plain stretching as far as the eye can see in every direction. In J. Weinstein and C. Colebrook (eds) Posthumous Life: Theorizing Beyond the Posthuman. New York: Columbia University Press, pp. 271-295.

Morton T. (2016a) Molten Entities. In D. Daou and P. Pérez-Ramos (eds) New Geographies 08: Island. Cambridge, MA: Universal Wilde, pp. $72-76$.

Morton T. (2016b) Dark Ecology: For a Logic of Future Coexistence. New York: Columbia University Press.

Morton T. (2013) Hyperobjects. Philosophy and Ecology after the End of the World. Minnesota, MN: University of Minnesota Press

Mortreux C. and Barnett J. (2009) Climate Change, Migration and Adaptation in Funafuti, Tuvalu. Global Environmental Change, 19(1): $105-112$.

Moten F. (2003) In the Break: The Aesthetics of the Black Radical Tradition. Minneapolis, MN: University of Minnesota Press.

Murray P. (2018) What is an Island? Art and archipelagic thinking in the $21^{\text {st }}$ Century. Circa: Art Magazine. 5 September, 2018. https: //circaartmagazine.net/what-is-an-island-art-and-archipelagic-thinking -in-the-21st-century/

Nakashima D., McLean C. G., Thulstrup H., Castillo A. M. and Rubis, J. (2012) Weathering Uncertainty: Traditional Knowledge for Climate Change Assessment and Adaptation. Paris, France: UNESCO.

Nancy J. L. (2014) After Fukushima: The Equivalence of Catastrophes. New York: Fordham University Press.

Neimanis A. (2019) The Weather Underwater: Blackness, White feminism, and the breathless sea. Australian Feminist Studies, 34(102): 490-508.

Neimanis A. (2018) Bodies of Water, Human Rights and the Hydrocommons. TOPIA: Canadian Journal of Cultural Studies, 21: 161-182.

Nicks D. (2017) Stark and Beautiful Photos of Resilience from the Storm-Battered U.S. Virgin Islands, Huffpost. https://www.huffpost .com/entry/stark-and-beautiful-photos-of-resilience-from-the -storm_b_59e1e361e4b003f928d5e6fd

Nimführ S. and Sesay B. (2019) Lost in Limbo? Navigating (im)mobilities and practices of appropriation of non-deportable refugees in the Mediterranean area. Comparative Migration Studies, 7(1): 1-19.

Nwanze K. F. and Sinon P. (2013) The Self-sufficient Seychelles Island that May Hold the Secret for Small Island States. Skift. AllAfrica 
.com. 5 March 2013. https://skift.com/2013/03/05/the-self-sufficient -seychelles-island-that-may-hold-the-secret-for-small-island-states

O'Brien S. (2017) Resilience Stories: Narratives of adaptation, refusal, and compromise. Resilience: A Journal of the Environmental Humanities, 4(2-3): 43-65.

O'Keefe P. and Conway C. (1977) Natural Hazards in the Windward Islands. Bradford Disaster Research Unit Occasional Paper 14. Bradford: University of Bradford.

Olwig K. R. (2007) Are Islanders Insular? A personal view. Geographical Review, 97(2): 175-190.

Papoutsaki E. and Harris U. S. (2008) Unpacking 'Islandness' in South Pacific Islands Communication. South Pacific Islands Communication: Regional Perspectives, Local, 219-234.

Paravisini-Gilbert L. (2015) Bagasse: Caribbean Art and the Debris of the Sugar Plantation. In E. DeLoughrey, J. Didur and A. Carrigan (eds) Global Ecologies and the Environmental Humanities: Postcolonial Approaches. New York: Routledge, pp. 73-95.

Parikka J. (2015) A Geology of Media. Minneapolis, MN: University of Minnesota Press.

Patino J., Whittaker R. J., Borges P. A., Fernández-Palacios J. M., Ah-Peng C., Araújo M. B., Ávila S. P., Cardoso P., Cornuault J., de Boer E. J. and de Nascimento L. (2017) A Roadmap for Island Biology: 50 fundamental questions after 50 years of The Theory of Island Biogeography. Journal of Biogeography, 44(5): 963-983.

Pelling M. and Uitto J. I. (2001) Small Island Developing States: Natural disaster vulnerability and global change. Global Environmental Change Part B: Environmental Hazards, 3(2): 49-62.

Percival G. S. (2008) An Assessment of Indigenous Environmental Knowledge (IEK) in the Pacific Region to Improve Resilience to Environmental Change. Climate Change Research Centre. Kensington, Australia: University of New South Wales.

Perez C. S. (forthcoming) Thinking (and Feeling) with Anthropocene (Pacific) Islands. Response to Chandler D. and Pugh J. (forthcoming) Anthropocene Islands: There are only islands after the end of the world. Dialogues in Human Geography.

Perez C. S. (2020a) 'The Ocean in Us': Navigating the blue humanities and diasporic Chamoru poetry, Humanities, 9(3), 66. DOI: https://doi .org/10.3390/h9030066 E-pub ahead of print: E-pub ahead of print https://www.mdpi.com/2076-0787/9/3/66

Perez C. S. (2020b) Habitat Threshold. Oakland, CA: Omnidawn Publishing. 
Peters K. and Steinberg P. (2019) The Ocean in Excess: Towards a more -than-wet ontology. Dialogues in Human Geography, 9(3): 293-307.

Petzold J. and Ratter B. M. (2015) Climate Change Adaptation Under a Social Capital Approach: An analytical framework for small islands. Ocean \& Coastal Management, 112: 36-43.

Pickett S. T. and Thompson J. N. (1978) Patch Dynamics and the Design of Nature Reserves. Biological Conservation, 13(1): 27-37.

Proctor J. D. (2013) Saving Nature in the Anthropocene. Journal of Environmental Studies and Sciences, 3(1): 83-92.

Pugh J. (2020) The Affirmational Turn to Ontology in the Anthropocene: A critique. In M. Stephens and Y. Martínez-San Miguel (eds) Contemporary Archipelagic Thinking: Toward New Comparative Methodologies and Disciplinary Formations. Lanham, MD: Rowman and Littlefield, pp. 65-83.

Pugh J. (2018) Relationality and Island Studies in the Anthropocene. Island Studies Journal, 13(1): 93-110.

Pugh J (2017) Postcolonial Development, (Non)sovereignty and Affect: Living on in the wake of Caribbean political independence. Antipode, 49(4): 867-882.

Pugh J. (2016a) The Relational Turn in Island Geographies: Bringing together island, sea and ship relations and the case of the landship. Social \& Cultural Geography, 17(8): 1040-1059.

Pugh J. (2016b) The Returning Terms of a Small Island Culture:

Mimicry, inventiveness and suspension. In E. Stratford (ed) Island Geographies: Essays and Conversations. Abingdon: Routledge, pp. 132-144.

Pugh J. (2014) Resilience, Complexity and Post-liberalism. Area, 46(3): 313-319.

Pugh J. (2013) Island Movements: Thinking with the archipelago. Island Studies Journal, 8(1): 9-24.

Pugh J. and Grove K. (2017) Assemblage, Transversality and Participation in the Neoliberal University. Environment and Planning D: Society and Space, 35(6): 1134-1152.

Puig de la Bellacasa M. (2017) Matters of Care: Speculative Ethics in More Than Human Worlds. Minneapolis, MN: University of Minnesota Press.

Quammen D. (2018a) The Tangled Tree: A Radical New History of Life. New York: Simon and Schuster.

Quammen D. (2018b) Blurring Life's Boundaries. Anthropocene. https://www.anthropocenemagazine.org/2019/06/blurring-lifes -boundaries 
Rakuita T. (2017) The Notion of 'Constellative Thinking' in Pacific Thought: Expanding Oceania. Pacific Dynamics: Journal of Interdisciplinary Studies, 1(1): 33-45.

Rankin J. R. (2016) Tracing Archipelagic Connections through Mainland Islands. New Zealand Geographer, 72(3): 205-215.

Rawlings A. (2020) Sound of Mull. Laboratory for Aesthetics and Ecology. https://issuu.com/echolology/docs/soundofmull-issuu1

Rawlings A. (n.d.) Sound of Mull, Angela Rawlings website. https: //arawlings.is/sound-of-mull

Raygorodetsky G. (2017) The Archipelago of Hope: Wisdom and Resilience from the Edge of Climate Change. New York: Pegasus Books.

Read L. E. [1958] (2019) I, Pencil. Atlanta, GA: Foundation for Economic Education.

Reid J. (2017) 'We the resilient': Colonizing indigeneity in the era of Trump. Institute for Interdisciplinary Research into the Anthropocene. 11 October 2017. https://iiraorg.com/2017/10/11/we-the-resilient -colonizing-indigeneity-in-the-era-of-trump

Riquet J. (2020) The Aesthetics of Island Space: Perception, Ideology, Geopoetics. New York: Oxford University Press.

Riquet J. (2016) Islands Erased by Snow and Ice: Approaching the spatial philosophy of cold water island imaginaries. Island Studies Journal, 11: 145-160.

Rist L., Felton A., Nyström M., Troell M., Sponseller R. A., Bengtsson J., Österblom H., Lindborg R., Tidåker P., Angeler D. G. and Milestad R. (2014) Applying Resilience Thinking to Production Ecosystems. Ecosphere, 5(6): 1-11.

Ritson K. (2020) The View from the Sea: The power of a blue comparative literature, Humanities, 9(3): 68. DOI: https://doi.org/10.3390/h9030068. E-pub ahead of print: https://www.mdpi.com/2076-0787/9/3/68

Roberts B. R. (2020) Reprise Editor's Introduction. Journal of Transnational American Studies, 11(2). E-pub ahead of print https://escholarship .org/uc/item/35z4q1mz

Roberts B. R. and Stephens M. A. (eds) (2017) Introduction. In B. R. Roberts and M. A. Stephens (eds) Archipelagic American Studies. Durham, NC: Duke University Press, pp. 1-54.

Robertson M. L. B. (2018) Crafting Certainty in Liquid Worlds: Encountering Climate Change in Kiribati. In T. Crook and P. Rudiak-Gould (eds) Pacific Climate Cultures: Living Climate Change in Oceania. Warsaw: De Gruyter Open, pp. 45-59.

Robin L. (2014) No Island is an Island: Islands are a natural laboratory for science, they gave us evolution. But isolation is no model for a 
cosmopolitan age, Psyche, 18 December 2014. https://aeon.co/essays /island-mindedness-has-no-place-in-a-cosmopolitan-age Rodriguez Coss, N. (2020) A Feminist Intersectional Analysis of Economic and Resource (In)equality in Puerto Rico Before and After Hurricane Maria, Gonzaga Journal of International Law, 23(1): 97-113.

Rolstad J. (1991) Consequences of Forest Fragmentation for the Dynamics of Bird Populations: Conceptual issues and the evidence. Biological Journal of the Linnean Society, 42(1-2): 149-163.

Ronström O. (2015) Island as Seriality. Paper presented at the Safeguarding and Promotion of Sea and Island Culture conference, Nha Trang, Vietnam, 5-6 January 2015.

Rowling M. (2018) Reinventing Islands: Sink or Swim? Islands innovate to thrive in a high-stress world. Zilient.org. Thomson Reuters Foundation. 23 July 2018. http://news.trust.org/shorthand/reinventingislands

Royle S. A. (2007) The Company's Island: St Helena, Company Colonies and the Colonial Endeavour. London: IB Tauris.

Rubow C. (2018) Woosh-Cyclones as Culturalnatural Whirls: The receptions of climate change in the Cook Islands. In T. Crook and P. Rudiak-Gould (eds) Pacific Climate Cultures: Living Climate Change in Oceania. Warsaw: De Gruyter Open, pp. 34-44.

Salick J. and Ross N. (2009) Traditional Peoples and Climate Change. Global Environmental Change, 19(2): 137-190.

Salinas-de-León P., Andrade S., Arnés-Urgellés C., Bermudez J. R., Bucaram S., Buglass S., Cerutti F., Cheung W., de la Hoz C., Hickey V. and Jíménez-Uzcátegui G. (2020) Evolution of the Galapagos in the Anthropocene. Nature Climate Change, 10(5): 380-382.

Salt K. N. (2017) Twilight Islands and Environmental Crises: Re-writing a history of the Caribbean and Pacific regions through the islands existing in their shadows. In J. Adamson and M. Davis (eds) Humanities for the Environment: Integrating Knowledge, Forging New Constellations of Practice. Abingdon: Routledge, pp. 55-57.

Samman N. and Charrière J. (2018) As We Used to Float. Berlin: K. Verlag. Schneider R. (2020) This Shoal Which is not One: Island studies, performance studies, and Africans who fly. Island Studies Journal, 15(2): 201-218.

Schneider-Mayerson M. (2017) Some Islands will Rise: Singapore in the Anthropocene. Resilience: A Journal of the Environmental Humanities, 4(2-3): 166-184.

Schwartz S. W. (2017) Temperature and Capital: Measuring the future with quantified heat. Environment and Society: Advances in Research, 8: 180-197. 
Searle A. (2020) Absence. Environmental Humanities, 12(1): 167-172. Searle T. (2019) Burning the Bull: The changing meanings of a harvest ritual in the Anthropocene. Performance Research, 24(6): 79-85.

Selkirk D. (2020) The Island with a Key to our Future. BBC Travel, 15 June 2020. http://www.bbc.com/travel/story/20200614-ascension -island-the-island-with-a-key-to-our-future

Serres M. (2011) Variations on the Body. Minneapolis, MN: Univocal. Shakespeare W. (2002) The Tempest. In D. Lindley (ed) The Tempest. Cambridge: Cambridge University Press.

Sharpe C. (2020) Christina Sharpe reading Kamau Brathwaite's Dream Haiti. https://www.youtube.com/watch?v=Vs5mJYw83fE

Sharpe C. (2019) Still Here. TOPIA: Canadian Journal of Cultural Studies, 40: 5-14.

Sharpe C. (2016) In the Wake: On Blackness and Being. Durham, NC: Duke University Press.

Sheller M. (forthcoming) Island Ceronomy and Submerged Worlds. Response to Chandler D. and Pugh J. (forthcoming) Anthropocene Islands: There are only islands after the end of the world. Dialogues in Human Geography.

Sheller M. (2020) Island Futures: Caribbean Survival in the Anthropocene. Durham, NC: Duke University Press.

Sheller M. (2018) Caribbean Futures in the Offshore Anthropocene: Debt, disaster, and duration. Environment and Planning D: Society and Space, 36(6): 971-986.

Sheller M. (2007) Virtual Islands: Mobilities, connectivity, and the new Caribbean spatialities. Small Axe: A Caribbean Journal of Criticism, 11(3): 16-33.

Sheller M. (2000) The New Caribbean Complexity: Mobility systems, tourism and spatial rescaling. Singapore Journal of Tropical Geography, 30(2): 189-203.

Siutaia H. (2020) Pacific Countries Consider Traditional Knowledge. Samoa Observer, 7 October 2020. https://www.samoaobserver.ws /category/samoa/72097?utm_content=buffer2d36a\&utm_medium =social\&utm_source=twitter.com\&utm_campaign=buffer

Skidmore M. Godfrey M. and Garnier A. (n.d.) Yes, but Why? Olafur Eliasson. wepresent https://wepresent.wetransfer.com/story/yes-but -why-olafur-eliasson

Small M. F. and Hunter M. L. (1988) Forest Fragmentation and Avian Nest Predation in Forested Landscapes. Oecologia, 76(1): 62-64. 
Smart Island World Congress. (2018) Program Agenda 2018. http://www .smartislandcongress.com/en/agenda-2018

Smart Island Journal (n.d.) Smart Singapore. Smartisland.com. http: //smartisland.com/singapore-the-smart-island-smart-nation/

Smith L. T. (2012) Decolonizing Methodologies: Research and Indigenous Peoples. New York: Zed Books.

Solana A. (2017) How do you Turn these Islands into a Living IoT lab? Just add 500,000 sensors. ZDNET, 8 December 2017. https://www .zdnet.com/article/how-do-you-turn-these-islands-into-a-living -iot-lab-just-add-500000-sensors

Somerville, A. T. P. (2017) The Great Pacific Garbage Patch as Metaphor. In B. R. Roberts and M. A. Stephens (eds) Archipelagic American Studies. Durham, NC: Duke University Press, pp. 320-338.

Spahr J. (2005) This Connection of Everyone with Lungs: Poems. Berkeley, CA: University of California Press.

Springer A. S., Turpin E., Einfeldt K. and Wolf D. (2017) Reverse Hallucinations in the Archipelago, Intercalations, 3. SYNAPSE - The International Curators' Network. Berlin: K. Verlag \& Haus der Kulturen der Welt.

Starc N. and Stubbs P. (2014) No Island is an Island: Participatory development planning on the Croatian Islands. International Journal of Sustainable Development and Planning, 9(2): 158-176.

Steinberg P. E. (2005) Insularity, Sovereignty, and Statehood: The representation of islands on portolan charts and the construction of the territorial state. Geografiska Annaler, Series B: Human Geography, 87: 253-265.

Steinberg P. and Kristoffersen B. (2017) 'The Ice Edge is Lost... Nature Moved it': Mapping ice as state practice in the Canadian and Norwegian North. Transactions of the Institute of British Geographers, 42(4): 625-641.

Stephens M. and Martínez-San Miguel Y. (eds) (2020) Contemporary Archipelagic Thinking: Toward New Comparative Methodologies and Disciplinary Formations. Lanham, MD: Rowman and Littlefield.

Stengers I. (2015) In Catastrophic Times: Resisting the Coming Barbarism. Open Humanities Press.

Stengers I. (2014) Thinking with Whitehead: A Free and Wild Creation of Concepts. Cambridge, MA: Harvard University Press.

Stengers I. (2008) A Constructivist Reading of Process and Reality. Theory, Culture \& Society, 25(4): 91-110. 
Stratford E. (2003) Flows and Boundaries: Small island discourses and the challenge of sustainability, community and local environments. Local Environment, 8: 495-499.

Stratford E., Baldacchino G., McMahon E., Farbotko C. and Harwood A. (2011) Envisioning the Archipelago. Island Studies Journal, 6(2): 113-130.

Strathern M. (2020) Relations: An Anthropological Account. Durham, NC: Duke University Press.

Strathern M. (2004) Partial Connections. Savage, MD: Rowman \& Littlefield.

Suliman S., Farbotko C., Ransan-Cooper H., McNamara E. K., Thornton F., McMichael C. and Kitara T. (2019) Indigenous (Im)mobilities in the Anthropocene. Mobilities, 14(3): 298-318.

Suwa J. (2007) The Space of Shima. Shima: The International Journal of Research into Island Cultures, 1(1): 1-14.

Svedin U. and Aniansson B. (1987) Surprising Futures: Notes from an International Workshop on Long-term World Development, Friibergh Manor, Sweden, January 1986. Stockholm: Swedish Council for Planning and Coordination of Research.

Teaiwa K. M. (2015) Consuming Ocean Island: Stories of People and Phosphate from Banaba. Bloomington, IN: Indiana University Press.

Teaiwa K. M. (2012) Choreographing Difference: The (body) politics of Banaban dance. The Contemporary Pacific, 24(1): 65-94.

Teaiwa K. M. (2011) Recovering Ocean Island. Life Writing, 8(1): 87-100. Teaiwa T. (2007) To Island. In G. Baldacchino (ed) A World of Islands: An Island Studies Reader. Charlottetown, Canada, and Luqa, Malta: Institute of Island Studies, University of Prince Edward Island and Agenda Academic, p. 514.

The Living Archive. (2020) Extinction Stories from Oceania. https://www .extinctionstories.org/2020/05/28/competition2020

Theobold S. (2018) Mining Banaba: Katerina Teaiwa talks mining phosphate \& decolonising modern anthropology. The Familiar Strange, 12 November, 2018. https://thefamiliarstrange.com/2018/11/12/ep-26 -katerina-teaiwa

Thompson L. (2010) Imperial Archipelago: Representation and Rule in the Insular Territories under US dominion after 1898. Honolulu, HI: University of Hawai'i Press.

Thompson Reuters Foundation News. (n.d.) In Focus: Reinventing Islands. Thomson Reuters Foundation News. https://news.trust.org /packages/reinventing-islands-sink-or-swim 
Triantis K. A. and Matthews T. J. (2020) Biodiversity Theory Backed by Island Bird Data. Nature, 579: 36-37.

Tsing A. L. (2015) The Mushroom at the End of the World: On the Possibility of Life in Capitalist Ruins. Princeton, NJ: Princeton University Press.

Tsing A. L., Bubandt N., Gan E. and Swanson H. A. (2017) (eds) Arts of Living on a Damaged Planet: Ghosts and Monsters of the Anthropocene. Minneapolis, MN: University of Minnesota Press.

Tsing A. L., Mathews A. S. and Bubandt N. (2019) Patchy Anthropocene: Landscape structure, multispecies history, and the retooling of anthropology: An introduction to supplement 20. Current Anthropology, 60(20): 186-197.

United Nations (2004) Living with Risk: A Global Review of Disaster Reduction Initiatives. New York: UN Publications.

United Nations Climate Change (2019) Mapping Exposure to Sea Level Rise: Tonga, Samoa, Vanuatu and Papua New Guinea. United Nations Climate Change. https://unfccc.int/climate-action/momentum-for -change/ict-solutions/mapping-exposure-to-sea-level-rise

Vaai U. L. and Casimira A. (eds) (2017) Relational Hermeneutics: Decolonising the mindset and the Pacific Itulagi. Suva, Fiji: University of the South Pacific Press and Pacific Theological College.

Vaka 'uta C. F. K., Vaka 'uta L. and Lagi R. (2018) Reflections from Oceania on Indigenous Epistemology, the Ocean and Sustainability. In S. Hessler (ed) Tidalectics: Imagining an Oceanic Worldview Through Art and Science. Cambridge, MA: MIT Press, pp. 127-133.

Vale C. (2018) Understanding Island Spatiality through Co-visibility. Shima: The International Journal of Research into Island Cultures, 12(1): 79-98. van Dooren T. (2014) Flight Ways: Life and Loss at the Edge of Extinction. New York: Columbia University Press.

Vitousek P. and Chadwick O. (2013) Pacific Islands in the Anthropocene. Elem Sci Anth, 1, p.000011. DOI: http://doi.org/10.12952/journal .elementa.000011

Viveiros de Castro E. and Danowski D. (2018) Humans and Terrans in the Gaia War. In M. de la Candena and M. Blaser (eds) A World of Many Worlds. Durham, NC: Duke University Press, pp. 172-203.

Waddell E. (1975) How the Enga Cope with Frost: Responses to climatic perturbations in the Central Highlands of New Guinea. Human Ecology, 3: 249-273.

Wakefield S. (forthcoming) The Possibility of Islands in the Anthropocene. Response to Chandler D. and Pugh J. (forthcoming) Anthro- 
pocene Islands: There are only islands after the end of the world. Dialogues in Human Geography.

Wakefield S. (2020) Anthropocene Back Loop: Experimentation in Unsafe Operating Space. Open Humanities Press.

Wakefield S. (2018) Infrastructures of Liberal Life: From modernity and progress to resilience and ruins. Geography Compass, 12(7): e12377. DOI: https://doi.org/10.1111/gec3.12377

Wakefield S. and Braun B. (2019) Oystertecture: Infrastructure, profanation and the sacred figure of the human. In K. Hetherington (ed) Infrastructure, Environment, and Life in the Anthropocene. Durham, NC: Duke University Press. Draft available at: https://www.academia .edu/37771363/Oystertecture_Infrastructure_Profanation_and_the _Sacred_Figure_of_the_Human.

Walcott D. (1998) What the Twilight Says. Boston, MA: Faber and Faber. Walshe R. A. and Stancioff C. E. (2018) Small Island Perspectives on Climate Change. Island Studies Journal, 13(1): 13-24.

Wang J. (2020) Oceanic Feeling and Communist Affect. RIBOCA2 Public programmes. https://www.youtube.com/watch?v=ma6y2IFDfUY

Watts L. (2018) Energy at the End of the World: An Orkney Islands Saga. Cambridge, MA: MIT Press.

Watts V. (2013) Indigenous Place-thought and Agency amongst Humans and Non-Humans (First Woman and Sky Woman go on a European World Tour!). Decolonization: Indigeneity, Education \& Society, 2(1): 20-34.

Wells H. G. (2005) The Island of Dr. Moreau. Stilwell, KS: Digireads.com Publishing.

West R. (n.d.) A Canary in the Climate Change Coal Mine: The island nation of Kiribati is disappearing into the sea. Documentary Channel. https://www.cbc.ca/documentarychannel/m/features/a-canary-in -the-climate-change-coal-mine-the-island-nation-of-kiribati-is-d

Westman W. E. (1986) Resilience: Concepts and measures. In B. Dell, A. J. M. Hopkins and B. B. Lamont (eds) Resilience in Mediterranean -type ecosystems. Tasks for Vegetation Science series, vol 16. Dordrecht: Springer, pp. 5-21.

Wetlands Wanderers. (2018) The Startling Adventures of RonR: Presentation at 'Islands of the World XVI', Terschelling Islands, Wadden Sea, Netherlands, 10-14 June 2018.

Whitehead A. N. (1985) Process and Reality (corrected edition). New York: The Free Press.

Whitehead A. N. (1968) Modes of Thought. New York: The Free Press. 
Whitehead A. N. (1967) Science and the Modern World. New York: The Free Press.

Whyte A. V. (2017). Preparing for the Next Hurricane Irma, or Harvey: How analytics and smart cities will help. Huffington Post, 11 July 2017. https://www.huffpost.com/entry/preparing-for-the-next-hurricane -irma-or-harvey-how_b_5a01cb19e4b085d72ae06d2b?utm _content $=63400251 \& u t m \_m e d i u m=$ social\&utm_source=facebook Wilson P. J. (1973) Crab Antics: A Caribbean Case Study of the Conflict Between Reputation and Respectability. Prospect Heights, IL: Waveland Press Incorporated.

Wolfe C. (2017) Of Ecology, Immunity, and Islands: The lost maples of Big Bend. In J. Weinstein and C. Colebrook (eds) Posthumous Life: Theorizing Beyond the Posthuman. New York: Columbia University Press, pp. 137-152.

Wu S., Chen R. and Meadows M. E. (2019) Evolution of an Estuarine Island in the Anthropocene: Complex dynamics of Chongming Island. Shanghai, PR China. Sustainability, 11(6921): 1-17.

Wynter S. (2003) Unsettling the Coloniality of Being/Power/Truth/ Freedom: Towards the Human, After Man, its Overrepresentation An argument. CR: The New Centennial Review, 3(3): 257-337.

Wynter S. (in conversation with K. McKittrick) (2015) Unparalleled Catastrophe for Our Species? Or, to give Humanness a Different Future: Conversations. In K. McKittrick (ed) Syliva Wynter: On Being Human as Praxis. Durham, NC: Duke University Press, pp. 9-90.

Xie B., Zhu X. and Grydehøj A. (2020) Perceiving the Silk Road Archipelago: Archipelagic relations within the ancient and 21st-Century Maritime Silk Road. Island Studies Journal, 15(2). E-pub ahead of print DOI: https://doi.org/10.24043/isj.118

Yalcinkaya G. (2019) Kelly Jazvac presents 'beautiful and horrific' plastiglomerate lumps at Milan Triennale, dezeen, $21^{\text {st }}$ April, 2019. https://www.dezeen.com/2019/04/21/kelly-jazvac-plastiglomerate -milan-triennale

Yusoff K. (2018) A Billion Black Anthropocenes or None. Minneapolis, MN: University of Minnesota Press.

Yountae A. (2016) The Decolonial Abyss: Mysticism and Cosmopolitics from the Ruins. New York: Fordham University Press. 



\section{Index}

A

Aboriginal 16, 74, 91, 98

absences 98, 135-136

acidification 115,131

$\operatorname{actant}(\mathrm{s}) \quad 111,114,116,117,129$, 152,184

activist(s) x, 35

Actor Network Theory 114, 129,140

adapt(s), adapative, adaptation, adaptability $8,11,12,13$, 41, 44, 46, 51, 52, 56, 61, 65, $70,101,106,123,124,126$, 139,183

Adger, Neil 43

aesthetic(s) 16, 83, 88, 98, 102, 162,167

affordance(s) 2, 5, 8, 9, 10, 12, $13,25,28,82,83,111,113$, $114,117,123,127,133$, 141, 148, 184, 191
Afro-Caribbean 89

Afro-pessimism 144

afterlife, afterlives $28,29,33$, $146,153,163,170,185$

Age of Enlightenment 80

Age of Entanglement $\quad 19,75,80$

Ahuahu (Great Mercury) Island 67

àina 158

Alaimo, Stacy $\quad 1,22,28,29,49$, 110, 131, 145, 172, 174-176, 181-182

Alexander, Rosie 36

algae 58, 107, 125

algorithm(s), algorithmic 27, 113

Allar, Neal 107

Allen, Craig R. 205

alterbiopolitics 94

Amami Islands 74

ambiguous edges 151

American Samoa 132 
America(s) 36, 76, 102 amplify, amplifies, amplification(s) $37,50-51,63,92,97,124$, $130,143,157$

Anderson, Ben 10

Annan, Kofi 114

Anote's Ark 25

anteater 24, 128-129

Anthropocene Islands project xii, xiv, 8, 187-192

anthropology, anthropologist(s)

$38,71,72,101,153,166$

anti-explorer method 89,99

Antillanité 92

Antilles 106

anti-normative 166

ant(s) $\quad 129$

Aotearoa (New Zealand) 121, 132

apocalypse, apocalyptic 1,53 ,

$$
64,85
$$

aquapelago(s) $\quad 16,71,82-86,99$

Arawak peoples 89

archipelagic, nature of the

Americas 36

'archipelagic turn' 36

archipelago, archipelagos,

archipelagic $18,35,61$, 90, 93, 94, 104

archive(s) 31, 130, 132, 147, 163,170

Aristotle's Law of

Noncontradiction 174

Arnall, Alex 143, 176

$\operatorname{art}(\mathrm{s}) \quad 71,84$

Ascension Island 81

Asner, Gregory 27

assemble, assembled, assemblage(s)

$8,38,58,71,78,91,100$,

126,136

Atlantic 95, 103, 176 atmosphere(s) 54, 91, 107, 131

atoll(s) 26, 47, 107

attune(s), attuned, attunement 14 , $77,78,90,96,97,112,163$

Australia 25, 74, 98, 121, 132

Austronesia 119, 122

autopoietic 56, 82, 134

B

Babbage, Charles 122

bacteria 174

Bahamas 37

Bahn, Paul 2

Baldacchino, Anna xiv

Baldacchino, Godfrey xiii, 3,4 , $6,8,10,16,20,36,38,72$, $90,110,114,123,138,166$

Balearic Islands 123

Baltic Sea 58

Banaba 156

*banua 119-122

Barad, Karen 2, 21, 23, 28, 29, $34,110,141,143,147-148$, $150,153-155,164-166,190$

Barbadian 32

Bardsey Island (Wales) 105

Barker, Joanne 176

Barnett, Heather 58-59

Barnett, Jon 45, 48, 58, 67

Bateson, Gregory $\quad 12,29,43$, 44,154

beach(es) 117, 152, 168-169, 176 becoming, become-other, becoming-other $9,15,24$, $25,34,41,44,51,53,54,56$, $60-62,61-63,66,70,129$, $153,170,183$

Beer, Gillian 6

behavioural fingerprints 125 
Benítez-Rojo, Antonio 36

Benjamin, Walter 93

Bennett, Jane 82

Benwell, Richard 25, 111

Big Bang 70,87

Big Bend National Park 133, 135,140

Big Data 27, 185

Bikini Atoll 107

binary, binaries $21,24,74,88$, 161-163

bioindicators 26, 119

biomes 139

biopolitics 94

bio-sensor(s), biosensing 125

Bird Rose, Deborah 3, 9, 14-16, $72,74,91,96,98-100,145$

birds $11,27,50,95-96,127$

Black Beach 168

blackness 30, 32-33, 160-161, 162,177

Black Studies $\quad 32,159,160,166$

blank space(s), islands as $\mathrm{xi}, 49$, $102,113,123$

body, bodies, bodily $31,131,137$, 139, 157-158, 160, 162, 192

Bongie, Chris 36

Bonnett, Alastair xiv, 36

Borgnino, Emanuela xiv, 31, 105, 157-158

bounce back 106

boundaries 165

Bradley, Joff 198

Brathwaite, Edward Kamau 5, $6,22,28,29,32-33,35$, 158-162, 164, 176, 186

Bratton, Benjamin 122

Braun, Bruce 126

breath, breathe(s), breathing 97 Breckwoldt, Annette xiv, 26
Bremner, Lindsay xiv, 36,83

Briguglio, Lino 10

Brinklow, Laurie xiii

Bubandt, Nils 38

Buddhists 78

Burgos Martinez, Elena xiv, 3, 165,192

Burns, Lorna $\quad 18,107-108$

Burrow Collective 127

C

Caliban 178

Cameron, Fiona 43

Campos-Pons, Maria 176

Camus, Guigone 3, 26, 111, 121

Canada 71, 82, 95

canary in the coalmine 21 , $110,113,114,116,125$, 132,185

Capellán, Tony 31,176

carbon 29, 146, 150

care $90,92-100,101,167,168$

Caribbean $17,32,90,92,93$, $104,158,168,175,176$

Car Nicobar Islands 105

Carroll, Scott 208

Carter, Paul 36

Cartesian 18, 29, 144, 173

Casimira, Aisake 119

cat(s) 11,50

causal, causality $7,21,23,24$, 26, 28, 29, 44, 70, 111, 114, $116,117,125,126,133$, $148,152,154,174$

Cavallo, Eduardo 27, 123 centralisation 13

Chandler, David $8,10,13,26$, $38,42,52,110,115,117$, $124,171,190$ 
chaos, chaotic $33,88,168$

chaosmotic 149

Chao, Sophie 139

Charrière, Julian 107

Chernobyl 18

chestnut 153-154

China $37,76,78,188$

Christianity 173

circuit(s) $\quad 73,106$

cities $79,97,124$

citizen(s) 124

Clark, Eric xiii, 36, 72, 90

Clark, Nigel $3,21,28,29,31$,

$117,141,143,156-157$,

165,174

Clément, Gilles $\quad 80,85$

climate change $20,21,45-48$,

$85,107,110,112,113,117$,

$121,131,146,155,156,172$, 179,185

coal 53,146

coastline(s) 27, 113, 151

co-dependency, co-dependencies

$2,51,78,131$

Colebrook, Claire $\quad \mathrm{x}, 2,3,34,38$, 167, 170, 183, 192

Cole, David 23, 173

Collins, Damian 36, 208

colonial, colonialism, colonise(s),

colonising 4, 17, 21-22,

32-33, 104, 110, 135,

$136,141,144,148,149$,

$155,157-159,162,168$,

$172,173,175-177,179$,

$181,185,192$

command and control $18,44,64$,

65,117

common background 73

commons 92 community, communities 46,48 , 56,121

complex, complexity,

complexities 128

computation 27, 58, 122

Condé, Maryse 5

Connell, John 36

conquer, conquerors 87

conservation 81,130

consumerism ix, 4, 22, 34, 37, 136

contamination(s) 58, 147

continents $33,66,90,97,103,119$

contingency, contingencies

60,62

Conway, Craig $\quad 12,49$

Cook Islands 132

coral(s), coral reef(s) $82,107,113$, 115,179

co-relate, co-relations, co-relational $22,75,111,137$

Coronavirus 174

Correlation(s), correlational xiii, 7, 20, 21-23, 37, 109-140, $152,153,161,171,177$, 181,184

co-shaping $19,76,81$

cosmological compass 119-121, 132, 136

Crane, Ralph xi, 3, 36, 103

creative, creativity, creativities 64 , $77,78,80,91,103$

creolisation $17,35,105$

critical fabulation 33,163

critical theory, critical theorist(s)

$73,187-188$

critique, paths of 169

cultivate(s), cultivation(s)

77,94

cybernetic(s) $29,44,82,87,154$ 
cyborg(s) 62,73

cyclone(s) ix, 4, 54, 127

Cyphers, Peggy 23, 37, 143,

172-173

D

dance(s) 31, 34, 157, 158

Danowski, Déborah $1,95,117$

Daou, Daniel $\quad 2,9,14,19,71,75$, 79-80

Darwin, Charles $5,8,10-12,44$, 49-51, 56, 57, 59, 65-66, $87,122,128,130,131,175$, $181,183,186$

Dash, Michael 18

da Silva, Denise F. 28, 29, 173, 176-177

datafied 117,123

data (see Big Data) 139, 174

Davis, Heather 199

Davis, Sasha xiv, 36, 192

Dawson, Helen 2

death work 149

decolonising, decolonisation 162

deconstruct, deconstructive, deconstruction, deconstructable 2

defracted poetics 147

de Graaf, Jan 95

Deleuze, Gilles 5, 100, 107-108

della Dora, Veronica 36

DeLoughrey, Elizabeth xi, xiii, $3,20,23,30-32,36,38,43$, $110,113,120,141,155-156$, $159,175,176$

Dening, Greg 36

Denis Island 48

Depraetere, Christian xiv, 166
Derrida, Jacques $\quad 2,5,107,133$, 134, 136, 186

desert islands 75,172

Désert, Jean-Ulrick 176

desert(s) 4, 102

design, designers $\quad 16,71,80,103$

de Sousa Santos, Boaventura

97, 100

De Souza, Roger-Mark 3, 26, $46,48,113,114,119$

detectors 113

de-theorise, de-theorising

166,170

de Vries, Herman 80

De Waal, Alex 174

diaspora, diasporic 121

Dickenson, Adam 144

Dierksmeier, Laura xiv

difference(s) 6, 130

differentiation, differentiating, differentiated $10,42,49$, $50,51,56,59,87,183$

digital 112, 123-124, 137

disaster(s) 113, 123, 125, 173

disjuncture 30,156

disturbance(s) 9, 15, 16, 19, $61,62,71-78,91,98$, 101,103

divergence(s) 11, 51

diversity, diversities 13,42 ,

49,50

Dodds, Klaus 36

Dolphijn Rick 198

Doma Lama, Phu 39

doom and gloom 48

Drabinski, John 18, 92-93, 107

Dreamings 74, 91

Drifters Project 37

duty, duties 72,168 
dynamic(s) 53, 63, 65, 66, 86, Energy Islands 53, 61 88-89, 119-121, 124, 127, Enn, Rosa $\quad$ xiv, 26 $129,130,133,135,139$, $155,162,176,183,192$ dystopian 53

E

Earth 20, 25, 90, 99, 122, 129, 148,157

Earth island 70

Earth Island, network 70 earthquakes 119 echolocation 178

ecoLogicStudio $3,37,57-59,172$ ecology, ecological, ecologies 4, $13,23,31,44,50,77,80,81$, $91,94,98,102,111,112,117$, $126,133-135,149,150,164$

Edmond, Rod 6, 33, 114

Edwards, Paul 122, 127

Einfeldt, Kirsten 219

Einstein, Albert 87

Electric Nemesis 62-64

El Hierro 48

Elias, Anna 3

Eliasson, Olafur $\quad 138,150-151$

Ellsmoor, James xiv, 13, 123

embedded, embeddedness 18 , 24, 88-89, 146, 167

embody, embodied, embodies

$18,22,24,28-29,32,34,80$, $88-89,109,110,128,134$, $137,148,156,157,159$, 161,162

emergent, emergences 11,12 , $16,58,61,70,72,91,98$, $103,126,133,137$

end of the world $1,5,57,61$, $110,127,180-187,186$ entangle, entangled,

entanglements $\mathrm{x}, \mathrm{xi}, 1,2$, $4,8,9,13,19,20,27,35$, $49,51,56,57,63,71,76$, $80,83,87,98,99,101,109$, $113,118,127,139,146$, $147,149,152,165,168$, $180,182-185,189$

episteme $35,114,115,133,142$, $144,148,149,155,157$, $169,180,181$

epistemology, epistemological, epistemologies xi, xii, 5, 19, 24, 35, 52, 109, 114, $133,136,155,164$

equilibrium(s) 42, 44, 96, 103

Eriksen, Thomas 2

Erlandson, Jon M. 4, 20, 23, 110,113

erotics 162

essence(s) 3, 24, 28, 43, 53, 92, $116,130,167$

ethnography $71,101,103$

Euclidian 8, 15, 20, 89

European Union 48

Evans, Brad 13, 52

Evans, Mike 36

(the) everyday 42

evolutionary tree 10

evolution(s), evolutionary 12 , 24-25, 50-51, 67, 81, 87, $111,112,129,131,136$, 152,153

experience, experiential $\quad 111,190$ experiment $(\mathrm{al})$, experimentation $4,16,65,71,72,75,86,88$, 91, 94-95, 96, 101, 106, $139,170,171-172$ 
extinction(s) $\quad 6,66,131,132,140 \quad$ Flenley, John 2

extractive, extraction $\quad 169,170 \quad$ Fletcher, Lisa $\quad$ xi, xiii, 3, 36, 103

flightways 132

flora and fauna $46,118,135$

$\mathbf{F}$

Fair, Hannah 119

Falefou, Tapugao 119

Fanon, Frantz 182, 188, 190, 191,192

Farbotko, Carol xiv, 3, 43, 119

Faris, Jaimey 155

Farrier, David 21, 23, 30, 34, 110, $141,143-146,150,153,154$, 164,166

Federation of Micronesia 132 feedback(s), feedback effects, feedback loops $\quad \mathrm{x}, 2,5,8-9$, $12,13,20,25,27,29,42,45$, 47, 51, 52, 56-59, 71, 80, 94, $96,100,101,113,123,130$, $132,142,154$

Fehimović, Dunja xiv feminism, feminist, feminized 30, 155-156

Fernando, Nishara 200 figuration 81

Fiji 127, 132 Flores, Tatiana 23, 105, 110, 175,176

flourishing 75, 192

Foley, Aideen xiv

Folke, Carl 208

fonua 120

Foo, Shawna 27

footprints 144, 145, 164

Forest Peoples Programme 26, 55, 111, 118

forests, island dynamics 127 ,

$$
\text { 153-154 }
$$

Foucault, Michel 144

fractal(s) 151, 176, 177

French Polynesia 132

frog(s) 130

Fukushima 173

fungi 91

G

Gaia $70,75,102,117$

Gaini, Firouz 39

Filmproduktion and Arte G.E.I.E. Galápagos 50, 51, 133

(Netflix Series 'Islands of Galle, Nadine 47

the Future') 48, 123

finch(es) 11

Finland 158

Finney, Ben 120

Gan, Elaine 211

Garden City 147

garden(s), gardening 78,80 , 89-90

First Peoples Worldwide 26,119 generous, generosity 88, 168

fish 27, 55, 125

Fish, Adam 139

Genz, Joseph 120

geography matters 5

Fitzpatrick, Scott 4, 20, 23,

geological sublime 170 110,113

fixed entities $28,43,74$

Ghezal, Amina xiv

Ghosh, Amitav 23, 34, 143

flat, flatten(s), flattening $\quad 51,161$

Ghosh, Sahana 105, 106 
ghost(s), ghostly $21,33,133,135$, Gumbs, Alexis 1 140, 145, 147, 153, 154, 163 Gumbs, David 176

Gillis, John $\quad$ xi, 3, 6, 35, 47, 79, Gunderson, Lance $\quad 43,44$ $152,173,174$

Giraud, Eva $\quad \mathrm{x}, 2,182-183$

Günel, Gökçe 38, 102, 103

Gutierrez, Laurent 171-172

giving-on-and-with 16,18 ,

71,184

Glissant, Édouard $\quad 3,5,6,16-18$, $31,34,35,38,71,72$, 87-88, 92-94, 99, 104-108, $165,168-169,184,186$ global warming $4,23,26,45,117$, $137,144,149,150,173-174$, 179,185

god's eye $30,64,155$

Goffman, Erving 52

Gómez-Barris, Macarena 37

Gordon, Lewis 127

governance $10,25,42,60,86$, $101,120,123,125,126$

Graham, Natalie R. 56, 67

grasp(s), grasping, (un)graspable xi, 1-2, 16-18, 20-21, 28, $35,44,52,57,59,70,76$, $82-84,97,101,109,110$, $114,136,147,184,189$

Graziadei, Daniel xiv, 36 Great Mercury Island 67 Great Pacific Garbage Patch island 145,171

Greece 47

green infrastructure 126

Greenland 150

Grosz, Elizabeth 51

Grove, Kevin xiii, 10, 13, 36, 52, 103, 192

Grove, Richard 3

Grydehøj, Adam xiii, 6, 10, 26, $113,114,123,138$

Guåhan (Guam) 31, 105, 132

Guattari, Félix $\quad$ 100, 107-108

\section{$\mathrm{H}$}

Hadfield, Michael 14,38

Haida Gwaii 9, 71, 82, 102

Haiti 90, 158-160

Hall, Elizabeth $\quad 23,114$

Hallward, Peter 107

Hamilton, Scott 2

Handley, George 3

Hanna, Elizabeth 25, 111

happy ending(s) 70

Haraway, Donna $1,4,9,38$, $56,60,72,73,81-85,103$, 115-116, 184

Harford, Tim 44

harmony, harmonious 61

Harney, Stefano 161

Harris, Lindsay 36

Harris, Usha Sundar 36

Hau'ofa, Epeli $\quad$ 5, 35, 105, 120

haunting(s) 28, 31, 33, 34, 37,

$133,135,136,142,143$,

145-147, 153, 154, 156,

$162-164,176,185$

Hawai'i 9, 97, 105, 132, 139, 158

Hawkins, Harriet 83

Hay, Pete 36

Hayward, Phil xiii, 3, 9, 14-16, $36,71,82-83,85,88,99$, 103,113

Hejnol, Andreas 29

helpless 3

Henly-Shepard, Sarah 200

Hennessy, Elizabeth 51

Herrington, Susan 9, 80, 103 
Hesiod 75

Hessler, Stefanie $\quad 32,36,158$

His Highness Tui Atua Tupua Tamasese Ta'isi Efi 55

Holbraad, Martin 137, 153, 166-167

hold(s), holding (Storiation) 21, $23,30,34,149,156,160$, 170,177

holistic 70,119

Holland, John 44

Holling, Crawford S. 12, 44

homeostatic 82

homogeneous, homogenising 7 , $10,51,70,128,169,182,183$

Hong, Gang 36

Hong, Sun-Kee xiv

Honshu 78

hope $42,69,98$

Huang, Hsinya 102

hubris $2,3,64,75,76,119$,

133,158

human/nature divide $\mathrm{x}, \mathrm{xi}, 1$,

$6,33,46,49,57,62,66,71$,

$73,74,82,110,122,181$,

183, 185

humble(s), humbling $\quad 75,136,173$

Hurricane Irma 123

hurricane(s) ix, 4, 26, 29, 46, $150,177,179$

hyperobject(s) $117,150,174$

I

ice $35,140,146,150-151$

iceberg 150

Iceland 48, 138

Ice Watch 150-151

I-Kiribati (Kiribati) 157

immanence, immanent xii, 59-65, $69,87,165,175,183,191$ impassable rift 151-152

inculcating 9,70

India $37,83,188$

in-difference, indifference 165 , 167,168

Indigenous $22,26-27,30,31$, $38,54,102,111-113$, $118-120,122,132,156$, $161,177,183$

individuation $10,59,183$

Indonesia $124,139,165$

infinite, infinitely, infinities xiii, $18,72,89,99,100,134-135$, $136,146,150,151,174$

Ingersoll, Karin 3, 36

Ingold, Timothy 24

instrumental, instrumentalise, instrumentalised, instrumentalising 13,70

insular 3, 79, 152

intensive, intensities, intensify, intensifies 78, 190

interact, interacting, interactive, interaction $\mathrm{x}, \mathrm{xii}, 22,29$, $41,42,44,45,47-51,56$, $59,65,69,70,86,87,100$, $109,129-130,135,136$, 141,148

inter-action $\quad 32,34,185$ interdependent, inter-dependent, interdependency, interdependencies 5, 24, $42,47,51-56,59,70,84$, $86,122,123,129,132,183$

Intergovernmental Panel on Climate Change 23, 111,114

Internet of Things 27, 123, 185 inter-relation(s), inter-relation(al) 20, 21, 22, 24, 33, 59, 90, $105,121,133,141,153,162$ 
intimate, intimacy, intimacies

$55,81,144$

intra-action (vs inter-action)

$32,148,185$

intra-active 34,176

intra-, rather than inter-

activity 148

intra-relation (vs inter-relation)

$22,32,136,157,159,161-162$

Isaacs, Jenny xiv, 36

islanding $\quad 9,38,90$

islandness $\mathrm{x}$

island studies $\quad x i i, x i v, 5,7$,

34-36, 83, 101, 171, 180, 187-192

isolate(d) $3,23,29,36,50,52$, $56,59,60,79,127,150$, $152,173-174$

Italy 153

J

Jakarta 124

Japan 74, 76, 173

Jazvac, Kelly 176

Jetñil-Kijiner, Kathy 28, 31, 36,

155-156, 164

Johnson, Elizabeth 125

Johnson, Jessica 177

Jolly Margaret 208

Jørgensen, Peter 25

Joseph, Jonathan 52

Joseph, May xiv, 36, 37, 52

Judaeo-Christian 31

Jue, Melody 175

K

Kahele, Moana 36, 139

Kaiser-Bunbury, Christopher

$$
3,12
$$

Kanaka Hawai'i 36
Kanaka Hawai'i Cartography 36

Kanaka Maoli 55

22, Kanaky (New Caledonia) 132

Kanngieser, Anja 118-119, 127

Karelse, Theun 139

Karides, Marina 39

Kava, Leora 31

Kearnes, Matthew 194

Kearns, Robin 36

Kelly, David 103

Kelman, Ilan xiii, 4, 8, 10, 12, 26, $47,113,114,123,138$

Kimmerer, Robin 142

King, Russell 209

King, Tiffany Lethabo 21-23, 28, 29, 32, 33, 36, 110, 141, 143, 158, 161-164, 165, 166

Kinney, Kealohanuiopuna 45, 46, 52

kinship(s) 74

Kirby, Vicky 29, 190

Kiribati $121,132,156,157$

Kirksey, Eben 130

Kisanga, Eliawony 10

Kitara, Taukiei 220

knot(s) 15

Koch Curve 151

Kohn, Eduardo 24, 127-129, 136

Kothari, Uma 143, 176

Kouremenos, Anna xiv

Kristoffersen, Berit 35

Kueffer, Christoph 3,12, 45, 46, 52

Kurki, Milja 70

L

$\operatorname{lab}(\mathrm{s})$, laboratories $\mathrm{xi}, 12,26$, $45,49,63,81,114,123$

Lagi, Rosiana 221

laissez faire 81

Lama, Phu Doma 39 
Last, Angela $\quad 15,38$

Latour, Bruno $\quad 1,6,54,75,85$, $114,116,117,137,140$

Lawrence, Jennifer xiv legacy, legacies $17,21-23,29$,

31-34, 110, 130, 135, 136, $144,146,149,154,156,158$, $159,162,164,175-176,185$

Le Guin, Ursula K. 103

Lewis, David 120

life $8,10,12,13,17,25,26,29$, 41-44, 59-65, 65, 69, 87, $111,112,121,122,127-132$

liminal, liminality, liminalities 5 , $6,37,155,164,169,171,178$, $180,183,186,191$

linear, linearity, linearities xiii,

$3,5,7,11,20,30,42,43$, $49,65,106,128,147,152$, 158,164

listen(s), listening 96

lithosphere 117

littoral 163,164

liveable 78

lively, liveliness $\quad$ 79-87

Living Archive: Extinction Stories from Oceania, The 132 $\operatorname{lizard}(\mathrm{s}) \quad 11$

Lobo, Michelle 103

local, locality, localities 125

lodestone(s) 115

Lokman, Kees 9, 103

loop(ed) $\quad 150$

Loughran, Glenn xiv, 36

Louis, Renee Pualani 36, 139

Lovelock, James 70, 75, 102

Lyell, Charles 122

M

Macdonald, Stacey xiv Macfarlane, Robert 113 machine(s) 122

Machine Wilderness 139

Madeira 48

maelstrom 89

magnetic 115

magnify, magnifies $\quad 12,128$

Māhina, Hūfanga 'Okusitino

119,120

mainland(s), mainland thinking $2,3,5,6,7,13,18,23,37$, $41,43,47,51,52,54,60,63$, $89,104,142,162,164,178$, $180,181,188,191$

Malinowski, Bronisław K. 3, 47

Man 2 (Wynter) 182-183, 192

managerial $15,42,69,71$

mangroves 92-93, 105

Manhattan island 126

Māori 55

mapping(s) 104, 113, 139

marginal, marginalise(d) 43, 79, 186

Marland, Pippa xiv, 105, 143

Marshall Islands 132, 147-148, 156

Martínez-San Miguel, Yolanda xiv, 36

Martinique 17,18

Marx, Karl 131, 140

Massumi, Brian 175

Mathews, Andrew 21, 23, 28, $34,38,50,142,153-154$, 164,165

McCall, Grant xiv, 166

McIver, Lachlan 25

McKittrick, Katherine 3, 104

McMahon, Elizabeth xi, xiv, 3, 6,47

McMichael, Celia 220

McMillen, Heather L. 2, 8, 13, 47

McNamara, Karen 200 
Mead, Margaret $\quad$ 5, 71, 72 measure(s), measuring 117, 141,151

Mediterranean 12

melancholic 18

Melanesia 45, 73

Melanesian cyborgs 73

Meloni, Greca xiv

melt(s), melting $\quad 150,178$

memory, memories $\quad 93,94,139$

Mentz, Steve 38

Merola, Nicole 106-107

microcosm(s) 114

Middle Passage $17,93,160$

migration(s) 36, 103

migratory gardens 80

Mika, Kasia xiv, 90

military, militarism $\quad 147,148$

$\operatorname{mineral}(\mathrm{s}) \quad 91,156,176$

Mitchell, June 5

mobilities, (im)mobilities 36, 119-120

mockingbirds 11

modern, modernity, modernist,

Moderns $\mathrm{x}, \mathrm{xi}, 1-4,6,7,9$, $10,18-23,26,30,35,39,41$, $42,44,49,50,58,60,63,70$, $71,74,77,84,85,91,94,96$, $99,102,110,111,116,118$, $127,133,136,137,142-144$, 148-150, 156-158, 164, 167, 169-170, 175, 177, 179, 185,186

molluscs 125

Moncada, Stefano xiv

Moore, Amelia 37, 103, 143

Moore, Charles 176

moose skulls 117

moral, morality, moralism 57 ,

$75,94,154,167-169$ more-than-human $\quad \mathrm{x}, 117$, 126,133

morphogenesis 58

Morton, Timothy $1,2,3,21,23$, $29,31,34,49,110,117,143$, 146, 149-153, 164-166, $173-174,175,182$

Mortreux, Colette 48

Moten, Fred 32, 160, 161, 177

Mouffe, Chantal xiv

mountains 4, 118

Mull 106

multidimension(al),

multidimensionalities 23 , $95,152,173$

multispecies $74,99,102$

$\mathbf{N}$

Nabobo-Baba, Unaisi 118

Nadarajah, Yaso xiv

Nancy, Jean-Luc 3

narrative(s), narration $3,37,49$, $64,66,129,145,163,174$

nature, 'Nature' 1, 6, 24, 26, 45, $55,57,59,61,76,86,110$, $119,129,134,138,165,176$, 181,182

Nauru 132

navigate(s) 55

Neimanis, Astrida 21, 28, 29,

$32,110,142,176-178$

neoliberal(ism) 69

network(s), networked, networking

$78,124-125$

Newton(ian) 8, 20, 44, 60

Nielsen, Helene 39

Nimführ, Sarah xiv, 36

Niue 132

non-anthropocentric 22,58 
non-human, nonhuman 24 , $58-59,74,78,83,94,115$, $138,145,191$

non-linear $11,34,51,128$ non-modern $7,10,20,22,34$, $35,72,73,79,101,106$, $116,119,141,170,180$

Northern Mariana islands 132 North Sea 95

novel ecosystem 81

nuclear ix, 4, 18, 19, 23, 25, 28, 107, $141,144,147,149,164,179$

Nuku'alofa 118

Nwanze, Kanayo 23, 48, 114

O

O’ahu 158

O'Brien, Susan 42, 66

Oceania 35, 66, 104, 105, 120

ocean(s), oceanic $\quad x, 26,32,54$, $90,118,150,155-156,159$, $160,175,177,178$

O'Keefe, Philip 12, 49

Olwig, Kenneth 3

onto-epistemology, onto-

epistemologies, onto-

epistemological 3,7 ,

19-23, 26, 27, 28, 32, 39, 84, $102,110,112,113,117,118$, $122,126,129,132,135,136$, $137,141,142,144,147-150$, $159,160,161,165,166,168$, $173,175,177,180,181,183$, $184,185,189,191$

ontology, ontologies, ontological xi-xiii, 5, 7, 8-9, 16, 19, 35, $38,42,44,48-52,60,62,65$, 69-108, 70, 71, 77, 83, 84-92, 100-101, 109, 121, 136, 137,
$140,144,148,153,161,164$, $180,181,184,189,190$

opaque, opacity 17,86

open-ended $\quad 80,91,103,153,187$

Ophrys apifera 25

order, ordering $9,62,66,93,130$, 149,172

$\operatorname{organism}(\mathrm{s}) \quad 25,29,43,91,125$, 139,152

Orkney $9,48,53,61-63,81$

Orkney electron 61,64 the other of Thought (Glissant) 88,99

oyster(s) 126

$\mathbf{P}$

Pacific $46,67,89,90,102$, 118,156

Pacific Islands 120-121

Page, Philip 194

Palau 132

Paljassaare Peninsula 57-58

Papoutsaki, Evangelia xiv, 36

Papua New Guinea 12, 132

para-ontological 160

Paravisini-Gilbert, Lizabeth 3

Parc Henri Matisse 80

Parikka, Jussi 122

Paris 150

particularism 79

Patchwork(s), Patchworking xiii, $7,8,9,14-19,20,31,37$, $38,42,65,66,69-108,110$, $136,165,171,181-184$

patchy 38

Patchy Anthropocene 38

paternalistic 48

pathway(s) ix, 44, 112, 113,

132,136 
pattern(s), patterned, patterning $20,22,24,121,130,132,135$

Pedersen, Morten $\quad$ 137, 153, 166

Pelling, Mark 52

penguins 117

Percival, Galumalemana 3, 26, $45,52,55,113,118$

Perez, Craig Santos xiv, 3, 14, $21,28,31,105,120,143$, $155,165,192$

Pérez-Ramos, Pablo 2, 9, 14, 19, 71,75

PetaJakarta 124

Peters, Kimberley xiv, 176

Petzold, Jan xiv, 8, 13

Pitcairn 132 plant(s) 55, 81, 90, 91, 118, 128, 139,153

plastic(s) $113,171,172,176$

plastiglomerate 176

poesis 82

poetics $18,88,104,147$

poet(s), poetry $\mathrm{x}, 9,14,22,32-33$, 90, 93, 94, 97-99, 101, 105, $118,155,162,166$

policymaking, policymakers $\mathrm{x}$, $14,23,25$

Portefaix, Valérie 171-172 positivist 146 posthuman $49,51,109,131,182$ postmodern 10 potential, potentiality, potentialities $2,41,60,82,99,123,165$ pragmatic(s), pragmatically 71,175

predictable $16,44,75,111,184$ presence(s) 135-136, 154 problem-solver, problem-solving $41,65,69,149,167$ process(es) 10, 13, 15, 19, 23, $25,45,51,54,55,59,70$,
$72,76-80,82,83,86$, 89-90, 93, 95, 97, 101-103, $110,111,114,120,126,129$, $130,133,134,135,146,192$ processual becomings 41,66 processual change 44 Proctor, James 3 productive, productivity, productivities 78 , 103,142

progress $4,10,11,42,46,50,60$, $70,91,99,180$

Prospero 178

psychic marronage $\quad 158$

Puerto Rico 177

Pugh, Jonathan $\quad \mathrm{xv}, 8,10,13,18$, $26,36,38,42,52,110,117$, $171,178,190$

Puig de la Bellacasa, María 94 pulse(s) 97, 99

Q

quality, qualities 117

Quammen, David 11,50, 54

quantity, quantities 117

quantum $21,29,136,142,147$, $148,154,155,185$

quotidian $\quad 42,72$

$\mathbf{R}$

rainforest(s) 127-128

Rakuita, Tuinawi $\quad 35,105,120$

Randall, Jim 47

Rankin, Jonathan 36

Ransan-Cooper, Hedda 220

Rapa Nui (Easter Island) 132

Ratter, Beate xiv, 8, 13

Rawlings, Angela 106

Raygorodetsky, Gleb $\quad 8,13,45$, 48,113 
refugee(s) 159-160

register(s), registering, registration(s) xiii, 23, 30, 109, 111-114, 119, 125, 126, 159

regularities xiii, $24,87,111,112$, $116,128,130,131,133$, 135,152

Reid, Julian $\quad$ xiv, 13, 43, 52, 117 'Relation' 17-19, 38, 168 relational ontology, relational ontologies $8-10,41,42$, $45,46,87,88,102,105$, $183,184,189,191$

relational space(s) 5, 36, 45, 123 remote sensing 27,113 replicable $22,63,112,122$ Resilience, reslience xii, 7-14, $20,37,38,41-67,69,70$, 77, 83-88, 94, 96, 97, 100, $101,106,110,121,123,130$, $136,165,171,172,181$, 183,184

resilire 43 responsivity, responsivities 60 , $78,115,117,126$ (re)storiate, (re)Storiation(s) 163 revitalisation 77, 92

Rhiney, Kevon xiv rhythms 26, 54, 55, 124

Rickards, Lauren xiv

Riquet, Johannes xiv, 3, 11, 35, 102 Ritson, Katie 103

Riveros-Iregui, Diego 194 river(s) $\quad 17,82,124$ Roberts, Brian Russell xiii, 9, $14,19,36,72,89,99,104$

Robertson, Beth xiv

Robertson, Maria 2, 27, 54

Robin, Libby $\quad 81,103,129$

Robinson Crusoe 13, 52, 143 rocks 91,173

Rodríguez-Coss, Noralis 39 romantic, romanticising $\quad 70,170$ Ronström, Owe 36

Ross, Nancy 26, 45, 47, 55, 111,118

Rotmeijer, Sanne xiv

Royle, Steve 3, 36

Rubow, Cecilie 54

ruins $18,19,85,91,93-94,105$

rupture(s) 30, 96, 160, 163

Ruskin, John 122

Russia 95

S

Saami 158

sacred 106, 151

Saint Lucia 17

Salick, Jan $\quad 26,45,47,55,111,118$

Salt, Karen $\quad$ xiv, 28, 176

Samman, Nadim 107

Sammler, Katherine xiv

Samoa 132

Samsø 48

sand $33,55,129,143,152,158$, $163,176,178$

Sanders, Todd 23, 114

Santo Domingo, Dominican

Republic 31, 176

satoyama $16,19,71,76,77,81$

savages 3,47

saving (the Other) 3,43

scale $23,48,58,61,64,66,79$, $82,92,98,105,110,123$, $146,149,150,168$

Schneider-Mayerson, Matthew 139

Schneider, Rebecca 163-164

Schwartz, Scott 117

science(s) 81 
sea level rise $46,113,121,149$, $150,178,179$

Searle, Adam 140

Searle, Tamara 31, 157-158

seas $24,90,105,121,159,176$

seascape epistemology 36

Seidel, Henrike 26

self-adaptive $\quad 45,182$

self-determined 63

self-making $\quad 56,60,65$

self-organising 69

self-regulating $8,9,61,62,64$, $72,75,77,80,82,85,86,101$

self-reliance 52

self-sufficient 53

Selkirk, Diane 81, 103

semiotic 129

sense(s), sensing, sensory, senseability $113,114,116,117$, $119,122,123,126,130,132$, 138,139

sensitive $25,61,94,111,112$,

122,133

separation(s) $6,11,21,50,81,148$ Serres, Michel 24

Sesay, Buba 36

severance 73

Seychelles 48

Shakespeare, William 178

shamanistic $31,34,158$

Sharpe, Christina $21-23,28-33$, $110,141,143,158-160,162$, $165,166,176,177$

Sheller, Mimi xiii, 9, 14, 15, 31, $36,38,72,89-90,143,157$, 158,192

Sherpa, Lakpa 55

shima 74,82

shimmer 98-99

shoal(s) 161-164

Shubin, Neil 131 signal(s), signalling 116,152

signs $20,21,26,96-98,100,111$, $119,128,129,137,143$, 152,174

simplicity $\mathrm{xi}$

Singapore $124,139,146-147$

Sinon, Peter $\quad 23,48,114$

Skellig Michael 173-174

slavery $30,33,104,144$

smart grid 63

smart islands $27,112,123$, 132,137

Smart Island World Congress 123

Smith, Linda Tuhiwai 139

Smith, Vanessa 6, 33, 114

Smits, Alice 139

snails 38

social media 27

soil(s) 91, 129, 153

Solomon Islands 132

solutionist $15,70,183$

Somerville, Alice Te Punga 172

sound(s), sounding(s), soundscape(s) 127

space(s), spatially, spatiality, spatialities 22,71

space-time(s) xiii, 8, 18, 20-22, $33,54,64,106,135$, 146,148

Spahr, Juliana $9,14-16,72,97$, 98, 106-107

speciation $11,49,51,56,132$

species $\quad 34,56-57,80,112,125$, $129,130,135,147,172,173$ spectres $136,142,143$

speculate, speculative, speculatively, speculation(s) 21, 22, $28,135,136,138,142,148$, 151-153, 158, 160, 164, $166,167,177,191$

speculative bricolage $33,153,163$ 
Springer, Anne-Sophie 24, 122,123

stability, stabilities $\quad 11,42,43,183$

Stancioff, Charlotte 25, 111

Starc, Nenad 36

Startling Adventures of RonR, The 95, 96, 99

Star Wars 173

staying with the trouble 9,16 , $60,62,64,85,89,101,184$

Steinberg, Phil xiv, 35, 36, 176

Stengers, Isabelle $75,117,189-190$

Stephens, Michelle xiii, 9, 14-16, 19, 36, 72, 89, 99, 105

stewardship 94

Storiation(s), Storiation (s) 'without the subject' xiii, 7, 20-23, 27-34, 37, 109, 110, 112, 132-135, 138, 141-177, 165-169, 181, 182, 184, 185

story, stories (versus Storiation)

148,154

Stratford, Elaine xiii, 36

Strathern, Marilyn 5,71-73, 156, 186

St. Simons Island, Georgia 163 Stubbs, Paul 36

subject/object $21,33,62,74,82$

submarine $84,175,176$

Suliman, Samid $\quad 3,26,111$, 119-122

Suomenlinna 158

Supertrees (Singapore) 146-147 surface effect(s) $\quad 109,126$

surfer 55

sustainability $10,42,60,66,113$

Suwa, Jun'ichiro $36,74,82$

Swanson, Heather A. 221

Sydney 130

symbiotic 84

symbol(s), symbolic $\quad 4,101,114$ sympoietic $56,82,84$

systems xii, 13, 24, 44-45, 59, $61,86,111,122,125,127$, 129, 133-135, 139, 144

Szerszynski, Bronislaw 3, 21, $28,29,31,141,143$, 156-157, 165

T

Taino 89

Tallinn 37

Tallinn Architecture Biennale 37,57

TARDIS 151

taste 96

te $a b a \quad 157$

Teaiwa, Katerina $\quad 31,118,156-157$

Teaiwa, Teresia Kieuea 3, 9, 14, $30,72,90,118,164,165$

technology, technologies, technological 42,177

telescoping $30,155-156,158$

telos, teleological $42,46,49,65$, $66,70,87,130,152-153$, 164,167

temperature 115,135

Terschelling Island 95,99

test, testing, testing out 4,24 ,

$25,107,113,123$

Thaman, Konai Helu 118

thermodynamics 44

thermometer $112,115,116$

Thompson, Lanny 36

thought of the Other

(Glissant) 88

tidalectics $\quad 32-33,36,159,161$

Tilos 47

time, temporally, temporality, temporalities $8,9,30,71$, $126,133,144$ 
Todd, Zoe 118

Tokelau 132

Tonga 132

top-down $13,42,46,64,65,122$

totality, totalities $18,74,88,118$, $135,151,173$

toxic, toxin 125,130

trace(s) $28,33,34,129,130,133$, $142,147,153,154,156,157$, 162-164

trance 31,158

transcendental 69,96

transformative $28,45,65,78,99$, 118,188

transformative planetary

condition(s) $\mathrm{x}, 15,139,188$

transparent, transparency, transparencies $\quad 86-87$

travel, travelling $24,27,73,91$

trees 29, 135, 153

Tsai, Huei-Min xiv, 36

Tsing, Anna 1, 3, 9, 14, 19, 38, $60,71,75-76,81,85,86$, $88,91-92,96,152$

Turpin, Etienne $\quad 199,211,219$

Tuvalu 25, 132

U

Uitto, Juha 52

uncanny 142,145

uncertain, uncertainty, uncertainties $88,99,173$ uncontrollable 86 unexpected $22,53,71,94,131$, 143,163

ungraspable $86,88,173,178$

uninhabitable 162

unintentional $71,77,149$
United Nations $\quad 27,43,114,155$

United States of America 78, 156

universal, universality, universalist, universalities $7,18,41,79$, $149,161,180$

universe 87,129

unpredictable, unpredictability

$46,71,75,82,93,101$, 174,179

US military 147

V

Vaai, Upolu Lumā 119

Vaka 'uta, Cresantia 45

Vale, Celina xiv, 36

valley(s) 4

vampire squid 175

van Dooren, Thom 24-25, 132,136

vanua 118

Vanuatu 132

van Western, Jeroen 95

variations $51,113,116$

Varma, Saiba 103

Venice 139

verb, islanding as $9,38,90$

vibrant, vibrancy $1,10,82$, 134, 165

vigilance 98

Virtual Island Summit, The 123

vitality, vitalities 86

Viveiros de Castro, Eduardo

95,117

Vlieland 47

Vodou loa 158

vulnerable, vulnerabilities 20 , 48, 106 


\section{W}

Waddell, Eric 12

Wadden Sea 95

Wakefield, Stephanie $\quad$ xiv, 10, 14, $38,42,52,85,126,192$

Walcott, Derek 5, 93-94, 106, 166

Wallis and Futuna 132

Walshe, Rory 25, 111

Wang, Jackie $\quad 21,28,32,34,141$, 160,161

Watanabe, Chika 103

Watts, Laura $1,2,9,16,20,25$, $26,52,53,61-64,70,75$, $77,81,113,114$

Watts, Vanessa 118 wave(s) 26, 54, 55, 93, 159 weather, weather forecasting 27 weave 17

Weinstein, Jami $\quad \mathrm{x}, 2,183,192$

Wells, H. G. 57

Westman, Walter 12

West Papua 132

West, the 6, 49, 162

Wetlands Wanderers $14,16,72$, 95, 96

Whitehead, Alfred N. xii, 16, $84,188,190$

whole island system $\quad 45,67$

Whyte, Alisa 27, 113, 123

Wilson, Peter 52 winds(s) 55

withdrawn $144,150,152$,

$$
167-169,174,178
$$

withdrawn (islander) $\quad 168,169$

Wolf, Daniela 219

Wolfe, Cary $\quad 2,21,23,29,34,49$, 54, 133 136, 140, 147, 150, 153,182

worlding $18,88,89,115,134$

worldly 138

world-making $10,15,63,64$, $71,72,89-91,93,94,97$, 184, 189

worldview(s) 26, 55, 89, 94, 104, $119,120,121,127$

Wynter, Sylvia 104, 181-182, 190, 192

$\mathbf{X}$

Xie, Baoxia 35

Xroads Praxis 177

Y

Yalcinkaya, Gunseli 176

Yountae, An 14, 23, 28, 32, 38, 105,176

Yusoff, Kathryn $\quad 1,3,38,117,143$

Z

Zhu, Xianlong 223 


\section{ANTHROPOCENE ISLANDS ENTANGLED WORLDS}

The island has become a key figure of the Anthropocene - an epoch in which human entanglements with nature come increasingly to the fore. For a long time, islands were romanticised or marginalised, seen as lacking modernity's capacities for progress, vulnerable to the effects of catastrophic climate change and the afterlives of empire and coloniality. Today, however, the island is increasingly important for both policy-oriented and critical imaginaries that seek, more positively, to draw upon the island's liminal and disruptive capacities, especially the relational entanglements and sensitivities its peoples and modes of life are said to exhibit.

ANTHROPOCENE ISLANDS: ENTANGLED WORLDS explores the significant and widespread shift to working with islands for the generation of new or alternative approaches to knowledge, critique and policy practices. It explains how contemporary Anthropocene thinking takes a particular interest in islands as 'entangled worlds', which break down the human/nature divide of modernity and enable the generation of new or alternative approaches to ways of being (ontology) and knowing (epistemology). The book draws out core analytics which have risen to prominence (Resilience, Patchworks, Correlation and Storiation) as contemporary policymakers, scholars, critical theorists, artists, poets and activists work with islands to move beyond the constraints of modern approaches. In doing so, it argues that engaging with islands has become increasingly important for the generation of some of the core frameworks of contemporary thinking and concludes with a new critical agenda for the Anthropocene.

JONATHAN PUGH is Reader in Island Studies, University of Newcastle, UK. He is the author of over 70 publications developing relational thinking with islands and, more recently, the figure of the island in the Anthropocene. He leads the 'Anthropocene Islands' initiative: https://www.anthropoceneislands.online.

DAVID CHANDLER is Professor of International Relations, University of Westminster. He edits the journal Anthropocenes: Human, Inhuman, Posthuman. His recent books include Becoming Indigenous: Governing Imaginaries in the Anthropocene (2019) and Ontopolitics in the Anthropocene: An Introduction to Mapping, Sensing and Hacking (2018).
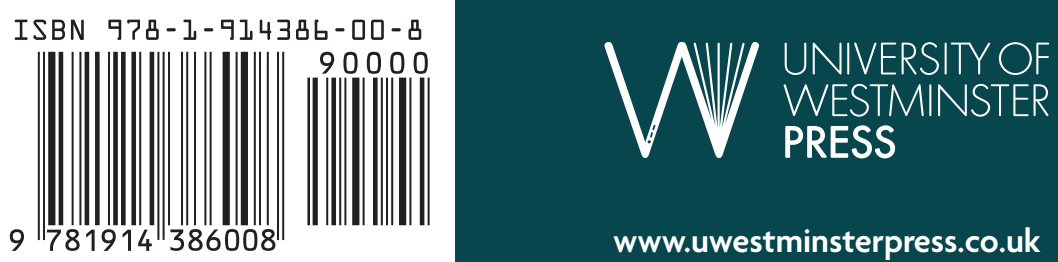